\title{
NUCLEATION PHENOMENA IN THE FORMATION OF ELECTRON-HOLE DROPS IN Ge
}

\begin{tabular}{|c|c|}
\hline hont Momo Woctomet & $\begin{array}{c}\text { RECENVED } \\
\text { LAWRENCE } \\
\text { REWXAIEY SARORATONY }\end{array}$ \\
\hline $\begin{array}{l}\text { bert Moore Westervelt } \\
\text { (Ph. D. thesis) }\end{array}$ & DEC $1 \quad 1977$ \\
\hline September 1977 & $\begin{array}{c}\text { LIBRARY AND } \\
\text { DOGUMENTS SECTION }\end{array}$ \\
\hline
\end{tabular}

Prepared for the U. S. Energy Research and

Development Administration under Contract W-7405-ENG-48

\section{For Reference}

Not to be taken from this room

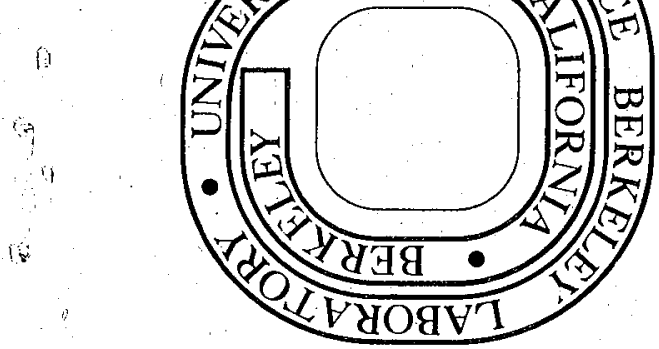



NUCLEATION PHENORENA IN THE

FORMATION OF ELECTRON-HOLE DROPS

\section{Table of Contents}

1 INTRODUCTION . . . . . . . . . . . . . . . . . . 1

11 Properties of the FE-EHD System . . . . . . . . . 5

12 Classical Nucleation Theory . . . . . . . . . . 11

13 Application of Nucleation Theory to EHD . . . . . . 17

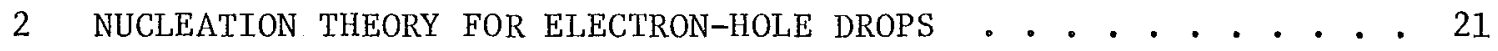

21 Rate Equations ................ 21

22 Size Distribution in Dynamic Equilibrium . . . . . . . 24

23 Free Energy Difference . . . . . . . . . . . 29

24 Calculation of Nucleation Rates . . . . . . . . . 32

25 Inhomogeneous Nucleation . . . . . . . . . . . 37

3 APPLICATION OF NUCLEATION THEORY TO OBSERVABLE PHENOMENA • • . . 41

31 Conservation Equation ............. . 43

32 Metastable Time Development ............. 46

33 Hysteresis and Thresholds ............. 50

34 Determination of the Surface Tension . . . . . . . 53

35 Modified Phase Diagram . . . . . . . . . . 55

36 Drop Radius . . . . . . . . . . . . . 59

4 EXPERIMENTAL APPARATUS . . . . . . . . . . . . . 63

41 Optical Excitation ................. 64

42 Germanium Samples . . . . . . . . . . . . 77

43 Signa1 Detection Apparatus . . . . . . . . . . 84 
5 EXPERIMENTAL RESULTS AND ANALYSIS . . • . . . . . . . . 90

51 Hysteresis Curves . . . . . . . . . . . . . 91

52 Metastable Time Development . . . . . . . . . 104

53 Effect of Impurities and Dislocations on Nucleation;

Surface Tension Measurement . . . . . . . . . . 111

54 Free-Exciton-Electron-Hole Drop Phase Diagram . . . . . 119

6 CONCLUSTONS . . . . . . . . . . . . . . . . . . 139

ACKNOWLEDGEMENTS . . . . . . . . . . . . . . . . . . . 143

REFERENCES . . . . . . . . . . . . . . . . . . . . . 144

TABLES . . . . . . . . . . . . . . . . . 158

FIGURE CAPTIONS . . . . . . . . . . . . . . . . . 163

FIGURES . . . . . . . . . . . . . . . . . 176 
NUCLEATION PHENOMENA

IN THE FORMATION OF ELECTRON-HOLE DROPS IN Ge

Robert Moore Westervelt

Materials and Molecular Research Division, Lawrence Berkeley Laboratory and Department of Physics, University of California

Berkeley, California 94720

ABSTRACT

A detailed theory of the nucleation of electron-hole drops (EHD) from a gas of free excitons (FE) in $\mathrm{Ge}$ is presented, together with a systematic experimental study of hysteresis and threshold phenomena in the luminescence of optically excited crystals of ultrapure Ge. The non-equilibrium rates of EHD formation and breakup and the concentration of EHD in dynamic equilibrium with the FE gas are calculated including the effects of the EHD surface energy $w$ and the EHD 1ifetime $\tau_{0}$. Large metastable and non-equilibrium effects are found. These results are used to quantitatively predict for Ge under continuous excitation 1) the EHD concentration and size vs. excitation level and history of excitation, 2) the metastable time development of the EHD concentration, 3) mathematically well-defined FE density thresholds $\mathrm{n}_{+}$ and $n_{\text {_ }}$ for the formation and destruction of EHD and thus the FE-EHD phase diagram including the effects of metastability and the EHD lifetime, 4) a procedure to accurately determine the EHD surface energy w from threshold data, and 5) limits to the steady-state EHD radius vs. temperature. Results for the calculated FE-EHD phase diagram for Si are also presented. Each calculation is made for two separate 
assumptions: homogeneous nucleation, and inhomogeneous nucleation of EHD on neutral impurity atoms. An experimental apparatus specially constructed to measure hysteresis and threshold phenomena in the luminescence of $\mathrm{EHD}$ and $\mathrm{FE}$ in $\mathrm{Ge}$ is described, including 1) a feedbackstabilized source of optical volume excitation for $\mathrm{Ge}$, which can be accurately and reproducibly cycled, and 2) a sensitive automated luminescence detection and signal averaging system. Experimental data obtained from ultrapure Ge are presented for 1) hysteresis curves for the EHD and FE 1uminescence intensity obtained by cycling the excitation at various temperatures between $1.2 \mathrm{~K}$ and $2.1 \mathrm{~K}$; including a procedure to accurately extrapolate the excitation thresholds 2) various experimental tests of the validity of EHD nucleation theory 3) the metastable time development of the EHD signal for very long times $\sim 4 \times 10^{3} \mathrm{sec}$ at temperatures between $1.25 \mathrm{~K}$ and $1.8 \mathrm{~K}, 4$ ) the effect of dislocations and shallow electrically active impurities on the excitation thresholds, and 5) a carefully calibrated phase diagram for the measured FE density thresholds $\mathrm{n}_{+}$and $\mathrm{n}_{-}$vs. T between $1.2 \mathrm{~K}$ and 2.0K. Excellent agreement between these data and nucleation theory is found. The principal experimental results and conclusions are 1) an estimate $\rho \sim 4.4$ for the EHD radiative enhancement factor, the ratio of the electron density at the position of a hole to the mean density, 2) logarithmic variation of the EHD signal with time and direct evidence that 1 uminescence hysteresis can exist for times as long as $6 \times 10^{4} \mathrm{sec}$, suggesting that EHD can be practically immobile under suitable circumstances, 3) the ineffectiveness of dislocations 
as nucleation centers for EHD in Ge and evidence for inhomogeneous nucleation on impurity atoms,4) the first correct measurement $\phi_{\mathrm{o}}=1.9 \pm 0.2 \mathrm{meV}$ of the EHD condensation energy from threshold data, resolving the previous discrepancy with spectroscopic measurements, and 5) an accurate measurement of the EHD surface energy, $w=(2.6 \pm 0.3) \times 10^{-4} \mathrm{erg} \mathrm{\textrm {cm } ^ { - 2 }}$. 



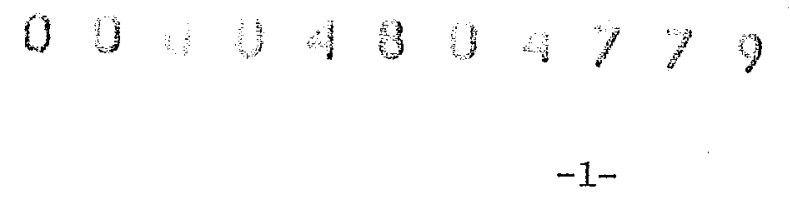

CHAPTER 1. INTRODUGTION

Norma1ly a crystal of Ge held at liquid helium temperatures is practically devoid of free carriers. However, when the crystal is excited by light, electrons and holes are created: the holes relax to the top of the valence band at the $(0,0,0)$ point in the Brillouin zone, and the electrons rapidly ( $\leqslant 1 \mathrm{nsec}$ ) relax to the indirect minimum in the conduction band at the $\left(\frac{1}{2}, \frac{1}{2}, \frac{1}{2}\right)$ point as shown in Fig. 1.1. The coulomb attraction of these carriers leads to the formation of a mobile bound state of an electron and a hole known as the Mott-Wannier free exciton ( $\mathrm{FE}$ ), analogous to a positronium atom. For low FE densities $n$, the free excitons are well separated, do not interact appreciably, and behave somewhat like an ideal gas. However, when a certain threshold value of $\mathrm{n}$ is exceeded (analogous to a saturated vapor pressure), drops of metallic electron-hole liquid (EHL) form. The existence of this novel state of matter was first suggested by Keldysh in $1968 .^{1.1}$ Since that time, the properties of electronhole drops (EHD) have been extensively studied, both experimentally and theoretically. This work has been the subject of a number of review articles, ${ }^{1.2-1.7}$ notably those by Hensel et a.1., ${ }^{1.6}$ and by Rice. ${ }^{1.7}$

The simplest and most direct method of experimentally investigating the properties of electron-hole drops in Ge is to study the recombination luminescence emitted by EHD and FE. Using the sensitive apparatus described in chapter 4 we obtained the luminescence spectrum shown in 
Fig. 1.2 from highly excited ultra-pure Ge at 2.00K. The signal-tonoise ratio of the largest peak in Fig. 1.2 is larger than $10^{5}$. Because the bandgap in Ge is indirect, radiative recombination at low temperatures must involve the emission of a phonon at the Brillouin zone edge to conserve crystal momentum. The principal features in Fig. 1.2 are three replicas of the EHD luminescence line at $729 \mathrm{meV}, 709 \mathrm{meV}$, and $701 \mathrm{meV}$ due to the emission of TA, LA and TO phonons as indicated. The LA phonon replica is used throughout this thesis both because it is the strongest, and because it is allowed by group theory. ${ }^{1.8}$ As indicated in Fig. 1.2, the LA phonon replica of the FE 1uminescence is also visible in the spectrum. Additional unresolved structure thought to be due to the emission of two phonons ${ }^{1.9}$ is also present in Fig. 1.2 for photon energies between 710 and $730 \mathrm{meV}$ and energies less than $700 \mathrm{meV}$. At lower energies the spectrum in Fig. 1.2 must be corrected for the response of the Ge photodetector used for the measurement (see Fig. 4.7).

The luminescence spectrum near $710 \mathrm{meV}$ obtained from the same Ge sample at $2.10 \mathrm{~K}$ for a much lower level of optical excitation is shown in Fig. 1.3. At this excitation level the EHD signal is entirely absent, and the only features visible in the spectrum are the LA and TO phonon replicas of the FE luminescence as indicated. When the excitation level is increased by a factor of 2.2 , the broad luminescence line near $709 \mathrm{meV}$ characteristic of EHD abruptly appears, as shown in Fig. 1.4. Thus there exists an excitation threshold for the creation of EHD. 
The line shapes and positions of the FE and EHD signals contain a good deal of information about the system. As indicated in Fig. 1.4, the lower energy edge of the FE line corresponds to the ground state energy $-\mathrm{E}_{\mathrm{x}}$ in Fig. 1.1, where $\mathrm{E}_{\mathrm{x}}$ is the $\mathrm{FE}$ binding energy. The intrinsic FE 1inewidth, unresolved in Fig. 1.4, is due to the thermal motion of excitons and is roughly proportiona1 to the temperature. At temperatures below $\sim 3 \mathrm{~K}$, the split-off $\mathrm{FE}$ ground state, which lies $\approx 1 \mathrm{meV}$ above the lowest ground state, is not appreciably populated and does not contribute to the lineshape (see section 11). For EHD the ground state energy $-\left(\phi_{\mathrm{e}}+\phi_{\mathrm{h}}\right)$ in Fig. 1.1 corresponds to the upper energy edge of the line; thus luminescence spectra can be used to measure the condensation energy $\phi=\left(\phi_{e}+\phi_{h}\right)-E_{x}$ of an e-h pair relative to the $\mathrm{FE}$ ground state as indicated in Fig. 1.4. The linewidth for EHD is equal to the sum of the electron and hole Fermi energies $\mathrm{E}_{\mathrm{F}}=\mathrm{E}_{\mathrm{F}}{ }^{\mathrm{e}}+\mathrm{E}_{\mathrm{F}}{ }^{\mathrm{h}}$ as indicated in Fig. 1.4, and can be used to determine the EHL liquid density. The spectral characteristic most important to the work presented in this thesis is that the 1ineshapes and positions for both FE and EHD are practically independent of the excitation level, and vary only slowly with temperature below $\mathrm{T} \sim 4 \mathrm{~K}$. Therefore the signals obtained with broad spectrometer slits $(\approx 2 \mathrm{meV}$ resolution) centered about $709 \mathrm{meV}$ and $714 \mathrm{meV}$ are accurately proportional to the average concentrations of $e-h$ pairs in the sample condensed as $\mathrm{EHD}$ and $\mathrm{FE}$ respectively, and can be used to study the variation of these concentrations with excitation level and temperature. 
While performing such an experiment in 1973, Lo, Feldman and Jeffries discovered that the EHD signals obtained by increasing and by decreasing the excitation to the same level were different, and that these signals displayed separate excitation thresholds. 1.10,1.11 They immediately realized that this hysteresis was clear evidence for gas-1iquid supersaturation effects such as those observed in cloud chambers, for example, but did not make a detailed comparison of their data with theory. A careful study of such phenomena can yield a great deal of information about the EHD-FE system. Supersaturation caused by the finite surface tension $\sigma$ of ordinary liquids is known to have a profound effect on the apparent gas-liquid phase diagram, particularly at low temperatures, and theoretical analysis of threshold data yields measurements of $\sigma$ and the condensation energy $\phi$. The e-h liquid, unlike ordinary liquids, is characterized by a finite 1ifetime which adds interesting and novel complexity to the system.

The work contained in this thesis constitutes a systematic experimental and theoretical study of supersaturation effects for the EHD-FE system in ultrapure Ge. We find excellent quantitative agreement between hysteresis and threshold data and nucleation theory, presented in chapters 2 and 3, which includes the effects of the finite EHL lifetime, and we are able to deduce accurate values for several important parameters, notably the surface tension $\sigma$ and the condensation energy $\phi$. 


\section{$00 \% 44004701$ \\ $-5-$}

\section{Properties of the FE-EHD System}

The simplest electronic complex present in excited crystals of Ge at low temperatures is the free exciton. Each exciton consists of an e-h pair bound by the Coulomb interaction. If the electron and hole bands were spherically symmetric, the binding energy $E_{x}$ of the ground state would be given by the simple hydrogenic expression

$$
E_{x}=\mu e^{4} / 2 K^{2} \hbar^{2}
$$

where $\mu$ is the reduced mass, and $k$ is the dielectric constant. The exciton mass would be the sum of the electron and hole masses and the degeneracy for Ge would be 32 , the product of the electron and hole degeneracies. Unfortunately, both the electron and hole bands in Ge are highly anisotropic. This anisotropy great1y complicates the theory, and leads to the creation of two types of excitons with different symmetry. Thus the ground state is split into two levels, separated by $\approx 1 \mathrm{meV}$, each with a degeneracy of 16 .

The most careful theoretical treatment of free excitons in Ge to date is that by Altarelli and Lipari. ${ }^{1.12}$ They find values of the ground state energy $\mathrm{E}_{\mathrm{x}}=4.18 \mathrm{meV}$ for the lower exciton and $E_{X}-\Delta=3.17 \mathrm{meV}$ for the upper exciton, where $\Delta=1.01 \mathrm{meV}$ 
is the ground state splitting. This calculation compares very well with the recent experimental values $\mathrm{E}_{\mathrm{X}}=4.15 \mathrm{meV}$ from far-IR photoconductivity $^{1.13}$ and $\Delta=1.01 \pm 0.03 \mathrm{meV}$ from absorption spectra. 1.14 Altare11i and Lipari also calculate the energy dispersion relation for the motion of entire free excitons and find that the variation of exciton energy with wavevector is strongly non-parabolic, ${ }^{1.12}$ as origina1ly suggested by Kane. ${ }^{1.15}$ Their calculated FE density of states is in excellent agreement with the measurements of Frova et a1.1.14 and yields values of the FE density-of-states effective masses near the energy minima of $\mathrm{m}^{*}=0.436 \mathrm{~m}_{\mathrm{o}}$ for the lower exciton and $\mathrm{m}^{*}=0.555 \mathrm{~m}_{\mathrm{o}}$ for the upper one. These parameters are of importance in the analysis of threshold data presented in chapter 5.

Excitons at high density in Ge can conceivably condense in a number of ways other than the formation of electron-hole drops. The most important of these is probably the formation of multi-exciton (ME) complexes (excitonic molecules); the density threshold for Bose condensation lies above that for the formation of EHD. Each ME complex consists of two or more excitons bound together by Coulomb forces; for example, a biexciton is analogous to a $\mathrm{H}_{2}$ molecule. Because the degeneracy of the bands is so large, the formation of complexes of more than two excitons should be possible before the exclusion principle becomes active, unlike the case for $H$. The stability of $\mathbb{M E}$ complexes, either free or bound to centers, is an important assumption in EHD nucleation theory, as discussed in chapter 2. 


\section{$00+400492$}

$-7-$

The first evidence for ME complexes was the observation of a series of sharp luminescence lines at lower energies than the FE line in 1ightly doped $\mathrm{Si}^{1.16,1.17,1.18}$ and $\mathrm{Ge},{ }^{1.19}$ which were attributed to ME complexes bound to impurity atoms. Subsequently this interpretation has been placed in doubt by the stress and Zeeman splitting measurements of Sauer ${ }^{1.20}$ and recently resurrected theoretically by Morgan $^{1.21}$ and by Kirczenow et a1.1.22 At this time the question of the existence of bound ME complexes is unresolved. However, Thomas et al. ${ }^{1.23}$ have very recently reported experimental evidence for free biexcitons in ultrapure Ge with the binding energy $\sim 0.2 \mathrm{meV}$ relative to two FE.

The various properties of electron-hole drops in Ge have been extensively studied for nine years. Rather than attempt to summarize all of this work here, we shall describe only the intrinsic characteristics of EHD in unstressed ultrapure Ge, free of externally applied magnetic or electric fields.

The EHL in $\mathrm{Ge}$ is a highly-degenerate $\left(\mathrm{E}_{\mathrm{F}}{ }^{\mathrm{e}}, \mathrm{E}_{\mathrm{F}}{ }^{\mathrm{h}} \gg \mathrm{kT}\right)$ Fermi liquid composed of both electrons and holes, which can move freely through the crystal lattice. Because the pressure exerted on the liquid by the FE gas at temperatures below $\sim 3 \mathrm{~K}$ is negligible, the density $n_{0}$ of $e^{-h}$ pairs in the liquid at a given temperature is a constant characteristic of the EHL. At $\mathrm{T}=0$, the 1iquid density is determined by minimizing the energy $-\mathrm{E}_{\mathrm{g}}$ per $\mathrm{e}-\mathrm{h}$ pair with respect to $\mathrm{n}_{\mathrm{o}}$, where $\mathrm{E}_{\mathrm{g}}$ is the binding energy relative to free carriers. 
App1ying the usual argument (see Ref. 1.7, for example) that the recombination of an electron and hole, each at the Fermi level, takes an $\mathrm{e}-\mathrm{h}$ drop from ground state to ground state (i.e., the EHD is not left in an excited state), we find that both the energy $-E_{g}$ and the chemical potential are given by the sum of the electron and hole work functions, i.e., $\mu=-\mathrm{E}_{\mathrm{g}}=-\left(\phi_{\mathrm{e}}+\phi_{\mathrm{h}}\right)$ at $\mathrm{T}=0$ as shown in Fig. 1.1. The condensation energy $\phi$ (FE work function) is defined as the difference between the chemical potential of the liquid and the lower $\mathrm{FE}$ ground state and is given by $\phi=\phi_{\mathrm{e}}+\phi_{\mathrm{h}}-\mathrm{E}_{\mathrm{x}}$. Thus the binding energy $\mathrm{E}_{\mathrm{g}}$ is measured from the high energy edge of the EHL luminescence 1ine. This situation is in contrast to that in simple metals, where the electron gas is confined under pressure, and the energies $-\mathrm{E}_{\mathrm{g}}$ and $\mu$ differ by $(2 / 5) E_{F}$, where $E_{F}$ is the Fermi energy. At finite temperatures, small $\mathrm{T}^{2}$ corrections to $\mu$ and $\mathrm{E}_{\mathrm{g}}$ can be calculated in a straightforward manner using Fermi gas theory. 1.7

Thus the electrons and holes in a Ge crystal excited above threshold separate into two phases, the $\mathrm{FE}$ gas and the constant density e-h 1iquid. Because the EHL possesses a finite surface tension $\sigma$, the liquid forms drops, presumed to be spherical, similar to those found in rainclouds, for example. However, unlike ordinary liquids the EHL has a finite lifetime $\tau_{0}$, so that the drop radius $\mathrm{R}$ is limited by the rate of collection of $\mathrm{FE}$ at the surface. ${ }^{1.2}$ Measured values of $\mathrm{R}$ are typically several $\mu \mathrm{m}$ at $2 \mathrm{~K} .{ }^{1.6}$ Thus we can picture the FE-EHD system in Ge under relatively low excitation as a fog of tiny spherical EHD surrounded by FE "vapor". Under much higher 
excitation other regimes analogous to the spray from a nozzle are possible, ${ }^{1.6}$ but will not be considered here.

Reliable values for most of the parameters of the EHL have been determined by careful analysis of luminescence spectra; we sha11 discuss on1y the most recent work. Thomas et a1. ${ }^{1.24}$ find for the condensation energy

$$
\phi=(1.8 \pm 0.2)+(2.2 \pm 0.9)(\mathrm{kT})^{2},
$$

where $\phi$ and $\mathrm{kT}$ are in $\mathrm{meV}$, and for the liquid density

$$
\mathrm{n}_{\mathrm{o}}=(2.38 \pm 0.05)\left[1-(0.94 \pm 0.10)(\mathrm{kT})^{2}\right]
$$

where $\mathrm{kT}$ is in meV and $\mathrm{n}_{\mathrm{o}}$ is in units of $10^{17} \mathrm{~cm}^{-3}$. These values agree with the earlier measurements of Lo. ${ }^{1.25}$ Using the value ${ }^{1.13}$ $E_{x}=4.15 \mathrm{meV}$ for the $\mathrm{FE}$ binding energy Eq. (1.1) yie1ds $\mathrm{E}_{\mathrm{g}}=5.95 \pm 0.2 \mathrm{meV}$ for the $\mathrm{e}-\mathrm{h}$ pair binding energy, which agrees very well with the most recent theoretical value ${ }^{1.26} \mathrm{E}_{\mathrm{g}}=5.9 \mathrm{meV}$. The density $\mathrm{n}_{\mathrm{o}}$ from Eq. (1.2) also agrees with the calculated value 1.26 $\mathrm{n}_{\mathrm{o}}(\mathrm{T}=0)=2.2 \times 10^{17} \mathrm{~cm}^{-3}$. Equations (1.1) and (1.2) are only valid well away from the critical point of the EHL, given by the measured values ${ }^{1.27} n_{c}=(0.8 \pm 0.2) \times 10^{17} \mathrm{~cm}^{-3}$ for the critical density and $\mathrm{T}_{\mathrm{C}}=(6.5 \pm 0.1) \mathrm{K}$ for the critical temperature. The EHL lifetime is apparently an intrinsic property of the liquid and is found to be $1.3,1.28$ $\tau_{0} \approx 40 \mu \mathrm{sec}$ from luminescence decay data in ultrapure Ge. One fundamental parameter of the EHL which had not been reliably measured before the work reported in this thesis is the EHD surface tension $\sigma$. 
In chapter 5 we present an accurate measurement $\sigma=(2.6 \pm 0.3) \times 10^{-4}$ erg $\mathrm{cm}^{-2}$ from threshold data. Preliminary accounts of this measurement were given in Refs. 1.29 and 1.30. The calculation of $\sigma$ for EHD is an important test of the surface theory of metals; the most careful theoretical treatments to date are those of Rose et a1. ${ }^{1.31}$ and of Vashishta et a1. ${ }^{1.32}$ who find respectively at $\mathrm{T}=0, \sigma=(2.4 \pm 0.5) \times 10^{-4}$ erg $\mathrm{cm}^{-2}$ and $\sigma=(3.5$ to 4$) \times 10^{-4} \mathrm{erg} \mathrm{cm}^{-2}$.

A second and equally important way to study the e-h liquid is to investigate the FE-EHD phase diagram obtained from measurements of the thresholds for drop formation vs. temperature. If one assumes that EHD and FE behave 1ike simple classical fluids, the threshold for drop formation is determined by the saturated vapor density:

$$
\mathrm{n}_{\mathrm{s}}=\mathrm{D}_{\mathrm{x}} \exp (-\phi / \mathrm{kT})
$$

where $D_{x}=\gamma_{x}\left(m * k T / 2 \pi \hbar^{2}\right)^{3 / 2}$ is the effective density of states with $\gamma_{x}$ the FE degeneracy. Equation (1.3) is obtained by simply setting the chemical potential of an ideal $\mathrm{FE}$ gas equal to that for the 1iquid. ${ }^{1.33}$ Using expressions similar to Eq. (1.3) to analyze onset threshold data between $2 \mathrm{~K}$ and $4 \mathrm{~K}$, several authors $1.2,1.10,1.34,1.35$ have obtained measurements of the condensation energy, typically $\phi \approx 1.5 \mathrm{meV}$, which disagree with the more reliable spectroscopic value. Furthermore, the thresholds measured below $2 \mathrm{~K}$ increasingly deviate from $\mathrm{Eq} \cdot(1.3)$ as $\mathrm{T}$ is decreased and appear to approach a temperature independent value. Neither of these effects was understood at the time of these experiments; the discrepancy between spectroscopic and threshold 


\section{4}

$-11-$

measurements of $\phi$ in particular was the subject of much speculation. The first proper explanation of this discrepancy as an effect of EHD nucleation was given by Westervelt, Staehli, and Haller. ${ }^{1.36}$

At the same time, in 1973 , Lo et a1. ${ }^{1.10}$ discovered the existence of hysteresis and metastable behavior in the luminescence of EHD in Ge. As discussed previously, they found that the EHD luminescence signa1 at a given excitation depended on the history of excitation of the sample, and that the apparent time for the system to reach equilibrium was very long ( $\gtrsim 300 \mathrm{sec})$. Furthermore, their observation that the thresholds for the formation and breakup of EHD differed by a factor of 2 to 3 indicated that the analysis of onset threshold data in terms of Eq. (1.3) was questionable. These observations motivated the experimental and theoretical analysis of supersaturation effects of the FE-EHD system presented in this thesis. We start with a discussion of classical nucleation theory for ordinary liquids such as water in the following section.

\section{Classical Nucleation Theory}

The theory of the nucleation of drops of ordinary liquids has been developed over the past 60 years. Classic treatments of nucleation theory have been given by Volmer, ${ }^{1.37}$ by Becker and Doring ${ }^{1.38}$ and by Zeldovich. 1.39 The subject has also been reviewed by McDonald 1.40 and by Abraham ${ }^{1.41}$ among others.

The basic physical process which causes metastability and supersaturation effects in the gas to liquid phase transition is the 
nucleation and growth of small embryonic liquid drops from the vapor. A macroscopic body of liquid is in equilibrium with its vapor at the saturated vapor density determined by equating the chemical potentials of the 1iquid and vapor, given by Eq. (1.3). However, such a body of liquid can only form by the growth of small clusters of vapor molecules. This growth is inhibited by the contribution to the total energy of a small drop due to the liquid surface tension $\sigma$. In order to maintain a drop of radius $R$ in equilibrium with the vapor, the chemical potential of the vapor must be increased above the saturated value by the amount $\Delta \mu$ determined by equating the Gibbs free energies per particle of the drop and the vapor.

$$
\Delta \mu=\Delta \mathrm{p} / \mathrm{n}_{\mathrm{o}}=2 \sigma / \mathrm{n}_{\mathrm{o}} \mathrm{R}
$$

where $\Delta \mathrm{p}=2 \sigma / \mathrm{R}$ is the additional pressure inside the drop due to the surface tension, and $n_{0}$ is the liquid density. Equation (1.4) implies that for a given supersaturated value of $\Delta \mu$, drops of a certain critical radius

$$
\mathrm{R}_{\mathrm{c}}=2 \sigma / \mathrm{n}_{\mathrm{o}} \Delta \mu
$$

will be in unstable equilibrium with the vapor. Drops of radius smaller than $R_{c}$ tend to shrink and disappear, whereas drops larger than $R_{c}$ tend to grow. Drops of radius $R>R_{C}$ can in principle grow to infinite radius; in practice either a macroscopic body of liquid is formed, or the final drop radius is limited by external mechanisms. Statistical fluctuations in the system lead to the formation of a finite number of 


\section{$00+44804765$}

embryonic drops of radius $R_{c}$. The fundamental problem of nucleation theory is the calculation of the concentration of embryos at the critical radius and thus the rate of formation of macroscopic drops.

We sha11 describe here a simple thermodynamic approach to this problem which contains most of the physical principles involved in more detailed calculations. We assume an ideal system free of contamination or external surfaces. The total difference in Helmholtz free energy between a drop of radius $\mathrm{R}$ and an equivalent number of vapor molecules is

$$
\Delta \mathrm{f}=4 \pi \mathrm{R}^{2} \sigma-(4 \pi / 3) \mathrm{R}^{3} \mathrm{n}_{\mathrm{o}} \Delta \mu
$$

For an ideal gas $\Delta \mu=\mathrm{kT} \ln \left(\mathrm{n} / \mathrm{n}_{\mathrm{s}}\right)$ where $\mathrm{n}$ is the vapor density and. $\mathrm{n}_{\mathrm{s}}$ is the saturated density from Eq. (1.3). This free energy difference is plotted for water at $300 \mathrm{~K}$ for several values of the supersaturation ratio $\mathrm{n} / \mathrm{n}_{\mathrm{s}}$ in Fig. 1.5. As indicated in Fig. 1.5, the quantity $\Delta \mathrm{f}$ has a maximum $\Delta \mathrm{f}_{\mathrm{c}}$ at the critical radius $\mathrm{R}_{\mathrm{c}}$ given by $\mathrm{Eq} .(1.5)$. As the supersaturation ratio $\mathrm{n} / \mathrm{n}_{\mathrm{s}}$ increases, both $\Delta \mathrm{f}_{\mathrm{c}}$ and $\mathrm{R}_{\mathrm{c}}$ decrease. Drops smaller or larger than the critical radius $\mathrm{R}_{c}$ respectively tend to shrink or grow in order to minimize the free energy of the system. Thus one can make an analogy between changes in drop radius subject to the potential $\Delta \mathrm{f}$ and the motion of particles in a potential well. The concentration $h(R)$ of drops of radius $R$ is given in thermodynamic equilibrium by

$$
h(R)=D \exp (-\Delta f / k T)
$$


where $\mathrm{D}$ is an effective density of states for drops (because $\mathrm{R}$ is quantized by the number of molecules in a drop; we need not use distributions for $D$ and $h$ ). Obviously Eq. (1.7) cannot hold for arbitrarily large radii, because $\Delta f$ decreases monotonically for $R>R_{c}$. However, inspection of Fig. 1,5 indicates that the free energy $\Delta \mathrm{f}_{\mathrm{c}}$ required to form a drop of critical size is very much greater than $\mathrm{kT}$ for smal1 supersaturation ratios. Thus the population of drops at $\mathrm{R}_{\mathrm{c}}$ from Eq. (1.7) is insignificant, and the collection of drops of radius $R<R_{c}$ is thermodynamically isolated from those of larger radii. Therefore the system is in a metastable state in which thermodynamic equilibrium holds for $R<R_{c}$ but not for $R>R_{C}$. As the supersaturation ratio $\mathrm{n} / \mathrm{n}_{\mathrm{s}}$ increases from one, the concentration of embryos of less than critical size increases until the rate at which embryos grow past the potential barrier $\Delta \mathrm{f}_{\mathrm{c}}$ at $\mathrm{R}_{\mathrm{c}}$ eventually becomes appreciable.

With these assumptions, the estimation of the formation rate $\mathrm{J}$ of macroscopic drops is straightforward. The rate $J$ is simply given by the product of $h\left(R_{c}\right)$, the equilibrium concentration of embryos at $R_{c}$, and $\left(\pi R_{C}^{2}\right) n \bar{v}$, the collision rate of vapor molecules with a drop of critical size, where $\bar{v}$ is the mean speed. Thus

$$
J=\pi R_{c}^{2} n \bar{v} D \exp \left(\frac{16 \pi \sigma^{3}}{3\left(n_{o} \Delta \mu\right)^{2} k T}\right)
$$

where the argument of the exponential is equal to $\Delta \mathrm{f}_{\mathrm{c}} / \mathrm{kT}$. The most striking feature of Eq. (1.8) is that the formation rate $\mathrm{J}$ is an 


\section{0}

extremely sensitive function of the supersaturation ratio $\mathrm{n} / \mathrm{n}_{\mathrm{s}}$. Assuming $\mathrm{D} \sim \mathrm{n}$, we can calculate $\mathrm{J}$ from $\mathrm{Eq} \cdot(1.8)$ for water at $300 \mathrm{~K}$ as shown in Fig. 1.5. The results are $\mathrm{J}=0$ for $\mathrm{n} / \mathrm{n}_{\mathrm{s}}=1$, Fig. 1.5a;

$\mathrm{J}=5 \times 10^{-41} \mathrm{~cm}^{-3} \mathrm{sec}^{-1}$ for $\mathrm{n} / \mathrm{n}_{\mathrm{s}}=2$, Fig. $1.5 \mathrm{~b} ; \mathrm{J}=5 \times 10^{13} \mathrm{~cm}^{-3} \mathrm{sec}^{-1}$ for $\mathrm{n} / \mathrm{n}_{\mathrm{S}}=5$, Fig. 1.5c; and $\mathrm{J}=8 \times 10^{19} \mathrm{~cm}^{-3} \mathrm{sec}^{-1}$ for $\mathrm{n} / \mathrm{n}_{\mathrm{S}}=10$, Fig. 1.5d; note that $\mathrm{J}$ increases by sixty orders of magnitude as $\mathrm{n} / \mathrm{n}_{\mathrm{s}}$ increases from 2 to 10 . The variation of the drop formation rate with $\mathrm{n}$ is so rapid that one can speak of a threshold vapor density $\mathrm{n}_{\mathrm{t}}$ at which J sudden1y "turns on". For water vapor at 300K, the threshold supersaturation ratio is $\mathrm{n}_{\mathrm{t}} / \mathrm{n}_{\mathrm{s}} \sim 5$. Thus measurements of the onsets $\mathrm{n}_{\mathrm{t}}$ for vapor condensation can differ greatly from the saturated density $\mathrm{n}_{\mathrm{s}}$ for ordinary liquids. Also, because the ratio $\mathrm{n}_{\mathrm{t}} / \mathrm{n}_{\mathrm{s}}$ decreases with increasing $\mathrm{T}$, the apparent value of the condensation energy obtained by applying Eq. (1.3) to the slope of $n_{t}$ vs. T is smaller than the actual value.

The discussion above applies on1y to an idea1 system free of impurities or external surfaces; this case is commonly referred to as homogeneous nucleation. In practice, the condensation of ordinary liquids is usually dominated by inhomogeneous nucleation, i.e. the formation of drops on various impurities or surfaces. Effects often associated with the inhomogeneous nucleation of ordinary liquids are the destruction of metastability and other supersaturation phenomena, and the limitation of the drop concentration by the density of nucleation centers. However, as we show in chapters 2 and 3 , these qualitative effects are not expected for the case of EHD in ultrapure Ge, and the 
differences between the results of homogeneous and inhomogeneous nucleation theory are primarily quantitative and surprisingly small. Finally, we discuss some modern developments in nucleation theory. The fundamental problem with the theoretical approach of Becker and Döring ${ }^{1.38}$ and of Zeldovich ${ }^{1.39}$ is that near threshold, the size of an embryo at the critical radius is so small that one questions whether this embryo can be treated as a drop of liquid; for water at $300 \mathrm{~K}$ the number of molecules in a drop of radius $\mathrm{R}_{\mathrm{c}}=0.61 \mathrm{~nm}$, Fig. $1.5 \mathrm{c}$, is only 32. If such an embryo behaves more like a molecular complex than a liquid, Eq. (1.6) for the free energy is inapplicable. Lothe and Pound $^{1.42}$ addressed this problem by making a detailed calculation of the free energy of small molecular complexes. Briefly, they found that the entropy associated with such complexes was expected to be much larger than for a liquid drop of the some size. Correspondingly, the formation rate $\mathrm{J}$ is much larger, and the calculated formation threshold $\mathrm{n}_{\mathrm{t}}$ for water decreases by rough1y $30 \% .^{1.41}$ However, the thresholds $n_{t}$ calculated for water from Becker and Döring theory agree quite well with the measured values, whereas those calculated with the Lothe-Pound theory do not. Empirically, most ordinarly 1iquids seem to fall in classes which agree with one theoretical approach or the other. Abraham ${ }^{1.41}$ has suggested that the difference between these classes is caused by molecular ordering in the 1iquid. In this case, we expect electron-hole drops to more nearly obey: the Becker and Döring theory, because the e-h liquid itself is a degenerate Fermi system, highly ordered in momentum space, rather than a molecular liquid. 
90,401097

$-17-$

\section{Application of Nucleation Theory to EHD}

In the preceding section we have shown how classical nucleation theory can qualitatively explain some of the threshold phenomena experimentally observed for EHD in Ge. Namely, the finite surface tension of drop embryos results in metastable states of the 1iquidyapor system in which the threshold $n_{t}$ for drop formation is several times the saturated value, and the apparent value of $\phi$ the condensation energy is smaller than the actual value. Because macroscopic drops of ordinary liquid are stable for vapor densities as small as $\mathrm{n}_{\mathrm{s}}$, the saturated value, classical nucleation theory also predicts hysteresis and separate thresholds for the: creation and destruction of drops. However, because the lifetime $\tau_{0}$ of EHD is not infinite, the quantitative application of the theory for ordinary liquids. to EHD is not justified a priori. In fact, classical nucleation theory cannot explain the large deviations from simple behavior observed in the FE-EHD phase diagram below $2 \mathrm{~K}$, nor does it predict the finite stable radius which is observed for EHD. In order to quantitatively analyze threshold data for EHD we have developed a theory of EHD nucleation, presented in chapters 2 and 3 , incorporating the e-h liquid lifetime, which predicts these phenomena among others.

To gain an understanding of the effect of the lifetime $\tau_{0}$ on nucleation theory, we consider the rate equation ${ }^{1.2}$ for the number of e-h pairs in a single electron-hole drop: 


$$
\frac{d}{d t}\left(\frac{4}{3} \pi R^{3} n_{0}\right)=\left(\pi R^{2}\right)(n \bar{v}-a)-\left(\frac{4}{3} \pi R^{3} n_{0}\right) / \tau_{0}
$$

Here $n_{0}$ is the density of e-h pairs in the EHL, $n \bar{v}$ and a are the coefficients of the rates of collection and evaporation of FE from EHD, with $\overline{\mathrm{v}}$ the mean FE speed, and the last term is the rate of volume decay. As we show in Chapter 2, the evaporation coefficient is given by

$$
a=n_{s} \vec{v} \exp \left(2 \sigma / R_{0} k T\right)
$$

where $n_{s}$ is the saturated density from Eq. (1.3), and the argument of the exponential contains the correction to the work function $\phi$ due to the surface tension. Equation (1.9) is easily solved in the steady state to yield the FE density $\mathrm{n}$ in dynamic equilibrium with an EHD of radius $\mathrm{R}$ :

$$
\mathrm{n}=\mathrm{n}_{\mathrm{s}} \exp \left(2 \sigma / \mathrm{Rn}_{\mathrm{o}} \mathrm{kT}\right)+\left(4 \mathrm{n}_{\mathrm{o}} \mathrm{R} / 3 \overline{\mathrm{v}} \tau_{\mathrm{o}}\right)
$$

For a given value of $\mathrm{n}, \mathrm{Eq} .(1.11)$ is an implicit expression for the drop radius $\mathrm{R}$. If $\mathrm{n}$ is sufficiently large Eq. (1.11) has two solutions: the smaller one is approximately equal to the critical radius $R_{c}$ from Eq. (1.5), and the larger one is approximately

$$
R_{S} \approx\left(3 \bar{v}_{0} / 4 n_{0}\right)\left(n-n_{s}\right)
$$


Inspection of Eq. (1.9) indicates that the solution near $\mathrm{R}_{\mathrm{c}}$ is unstable as for ordinary liquids whereas that near $\mathrm{R}_{\mathrm{S}}$ is stable. Thus this analysis reproduces the result of classical nucleation theory, but also predicts a stable radius $\mathrm{R}_{\mathrm{S}}$ determined by the EHL lifetime. The stable radius $R_{S}$ is simply a result of the balance of the net rate of FE collection of the drop surface and the rate of volume decay.

Another new result predicted by Eq. (1.11) (independently obtained by Silver ${ }^{1.43}$ ) is the existence of a minimum value $n_{m}$ of the $\mathrm{FE}$ density greater then $n_{s}$ for the existence of drops. At relatively high temperatures $(\mathrm{T} \gtrsim 2 \mathrm{~K}$ ) the first term in $\mathrm{Eq} .(1.11)$ is much greater than the second, and the minimum density $\mathrm{n}_{\mathrm{m}}$ is approximately equal to $\mathrm{n}_{\mathrm{s}}$ for macroscopic drops, as for ordinary liquids. However, $\mathrm{n}_{\mathrm{s}}$ decreases exponentially with temperature, and for $\mathrm{T}<2 \mathrm{~K}$, the second term in Eq. (1.11), due to the 1ifetime $\tau_{0}$, dominates and leads to hugely supersaturated values of $\mathrm{n}_{\mathrm{m}}$, which vary only slowly with temperature. The origin of this effect can be clarified by solving Eq. (1.12) for $n$, assuming $n \gg n_{s}$; we find

$$
\mathrm{n}=\mathrm{n}_{\mathrm{o}} \mathrm{R}_{\mathrm{s}} / 3 \overline{\mathrm{v}} \tau_{\mathrm{o}}
$$

which is just the second term in Eq. (1.11). Thus, if the EHD stable radius possesses a minimum value, the $\mathrm{FE}$ density for which drops are stable also has a minimum value, given by $\mathrm{Eq}$. (1.13). The minimum value of $R_{S}$ is in turn determined by the effect of the surface tension $\sigma$ on the drop radius in dynamic equilibrium, as for the critical radius $\mathrm{R}_{\mathrm{c}}$. The interplay of these effects is considered in detail in chapter 2. 
Again, this analysis reproduces the result of classical nucleation theory at high $\mathrm{T}$, but also predicts new phenomena below $\mathrm{T} \sim 2 \mathrm{~K}$ caused by the EHL lifetime.

The full theory of the nucleation of EHD in ultrapure Ge is developed in chapters 2 and 3 for both the homogeneous and inhomogeneous cases. The calculated thresholds $n_{+}$and $n_{-}$for the creation and destruction of EHD increasingly deviate from the saturated density $\mathrm{n}_{\mathrm{s}}$ at temperatures below $2 \mathrm{~K}$ as described above, but join at $\mathrm{T} \approx 1.3 \mathrm{~K}$. Below this temperature hysteresis and metastability in the FE-EHD system are predicted to vanish. Threshold curves for the EHD signal vs. excitation are also calculated, as is the metastable time development of the EHD concentration and the variation of the EHD stable radius with temperature and history of excitation.

In chapter 5 we present the results of a systematic study of nucleation phenomena manifested in the luminescence of EHD and FE in ultrapure Ge. Hysteresis curves for the EHD and FE signals vs. excitation are measured for temperatures between $1.2 \mathrm{~K}$ and $2.1 \mathrm{~K}$, and are used to determine the threshold phase diagram $\left(n_{+}\right.$and $n_{-}$vs. $T$ ) and the metastable time development of the EHD signal for times $\sim 4 \times 10^{3}$ sec. In addition, an investigation is made of the effects of impurity type and concentration and of dislocation density on the thresholds $n_{+}$and $n_{-}$, in order to determine the effectiveness of these defects as nucleation centers. All of the data obtained are in good quantitative agreement with the predictions of EHD nucleation theory. A careful fit of the phase diagram measured below $2 \mathrm{~K}$ to theory yields accurate values of the EHD surface tension $\sigma$ and the condensation energy $\phi$. 
CHAPTER 2. NUCLEATION THEORY FOR ELECTRON-HOLE DROPS

In chapters 2 and 3 we present a general theory of EHD nucleation for a spatially uniform system, with the following features:

1) quantitative prediction of hysteresis providing a procedure for data reduction to accurately determine the surface tension;

2) quantitative explanation of metastable time behavior; 3) quantitative explanation of the FE-EHD phase diagram which can be used to extract the condensation energy $\phi ; 4)$ prediction of EHD radii near both thresholds as functions of the temperature; and 5) calculation of the above results for drop formation in the pure $\mathrm{FE}$ gas (homogeneous case) and on impurity atoms (inhomogeneous case). In this chapter we derive explicit expressions for the non-equilibrium rates of formation $\mathrm{J}_{+}$and breakup J_of drops, and for the size distribution of drops in dynamic equilibrium. In chapter 3 we apply these results to experimentally measurable phenomena in $\mathrm{Ge}$ and $\mathrm{Si}$. The contents of this chapter have been previously published. ${ }^{2.1}$

\section{Rate Equations}

The basis of our calculation of the rates of EHD formation and breakup, $J_{+}$and $J_{-}$, is the kinetic approach of Becker and Döring. 2.2 Rather than using the thermodynamic approach to find the density of critical embryos (see Chapter 1 ), they solved rate equations describing the collisions between vapor molecules and drops. The physical situation we assume is the following: Within a spatially uniform 
FE gas, drops containing $v e-h$ pairs are formed from free or bound excitons by successive collisions of the type

$$
\operatorname{EHD}(\nu-1)+\mathrm{EE} \rightarrow \operatorname{EHD}(\nu)
$$

We assume that "drops" of all sizes $\nu>1$ are energetically stable and are therefore able to participate in the chain of collisions leading to larger drops. Because the concentration of embryos and small complexes is usually quite low, we ignore collisions in which two or more embryos coalesce. (At temperatures $\lesssim 1 \mathrm{~K}$ for $\mathrm{Ge}$ and $\lesssim 3 \mathrm{~K}$ for $\mathrm{Si}$, the predicted drop size becomes quite sma11 and this need no longer be true, see chapter 3.) For the inverse reaction

$$
\operatorname{EHD}(\nu) \rightarrow \operatorname{EHD}(\nu-1)+\mathrm{FE}
$$

drops release free excitons by thermionic emission ("evaporation"). In contrast to classical liquids, the EHD size $\nu$ can also be reduced by the recombination of an $\mathrm{e}-\mathrm{h}$ pair within a drop.

The rates of these three processes determine in general the time evolution of both the size distribution $\mathrm{g}_{v}$ (drops $\mathrm{cm}^{-3}$ ) and the net rate $J_{v}$ (drops $\mathrm{cm}^{-3} \mathrm{sec}^{-1}$ ) at which drops grow from size $(\nu-1)$ to $\nu$. By adding the volume decay rate $\nu / \tau_{0}$ of an EHD of size $\nu$ to the rates of $F E$ collection $\beta n \nu^{2 / 3}$ and evaporation $\alpha_{\nu} \nu^{2 / 3}$, we construct a set of rate equations similar to those of Becker and Döring: ${ }^{2}{ }^{2}$

$$
\begin{gathered}
J_{\nu}=g_{\nu-1} \beta n(\nu-1)^{2 / 3}-g_{\nu}\left(\alpha_{\nu} \nu^{2 / 3}+\nu / \tau_{o}\right) ; \\
d g_{\nu} / d t=J_{\nu}-J_{\nu+1} .
\end{gathered}
$$


In $\mathrm{Eq} .(2.1)$ we have $\beta \equiv \pi \mathrm{r}_{0}^{2}(8 \mathrm{kT} / \pi \mathrm{m} *)^{1 / 2}$, where $\mathrm{r}_{\mathrm{o}} \equiv\left(3 / 4 \pi \mathrm{n}_{\mathrm{o}}\right)^{1 / 3}$ is the Wigner-Seitz radius, $n_{o}$ is the density of e-h pairs within the liquid, and $m^{*}$ is the FE effective mass; $n$ is the FE density; $\tau_{0}$ is the total EHD lifetime; and the temperature-dependent evaporation coefficient $\alpha_{v}$ will be derived below. Hensel and Phillips ${ }^{2.3}$ have reported evidence for an additional temperature-independent evaporation rate $c v^{2 / 3}$ which should be added to $\alpha_{\nu} v^{2 / 3}$ in $\mathrm{Eq}$. (2.1). The most probable origin of this effect is emission of FE by excitations created by the volume decay of $e^{-h}$ pairs in a layer near the surface of a drop. Because the measured value $\mathrm{c} \sim 10^{5} \mathrm{sec}^{-1}$ (see chapter 5) corresponds to a surface layer only $\sim 15 \mathrm{~nm}$ thick, this term is proportional to the surface area even for sma11 EHD embryos.

Although Eqs. (2.1) and (2.2) could in principle provide a detailed description of the time development of drop nucleation and growth, their general solution is impractical. We shall consider only the steady state, defined by $\mathrm{dg}_{\mathcal{v}} / \mathrm{dt}=0$, where the net rate of drop formation $J_{V}=J=J_{+}-J_{-}$is constant in time and size independent. Dynamic equilibrium is defined by the additional condition $J_{+}=J_{-}$. As we shall see, steady-state solutions for $J_{+}$and $J_{-}$can be applied to time dependent problems if the size distribution $g_{V}$ satisfies the above restrictions for only a limited range of $\nu$ about the critical size.

The arguments presented above and the rate Eqs. (2.1) and (2.2) apply equally well to both homogeneous and inhomogeneous nucleation. The difference between the two cases arises in the effect of a nucleation center on the thermodynamic potential of a drop. In section 22 
through 24 we calculate the nucleation rates $J_{+}$and $J_{-}$, and the size distribution in dynamic equilibrium for homogeneous nucleation, then in section 25 modify the calculation where necessary to describe the inhomogeneous case.

\section{Size Distribution in Dynamic Equilibrium}

For the model of homogeneous nucleation, drops of e-h liquid nucleate in a $\mathrm{FE}$ gas free of nucleation centers and can rotate and move about within the crystal. Because the radii of embryos are much smaller than the FE mean free path, it is permissible to treat the drops of a given size $\nu$ as an ideal gas. We can thus picture the FE-EHD system as a collection of interpenetrating ideal gases identified by their sizes $\nu$, which interact with each other via $e-h$ recombination and by the absorption and emission of excitons.

In order to calculate the EHD size distribution in dynamic equilibrium, denoted by $h_{v}$, for this system, we first need to find an expression for the thermionic emission coefficient $\alpha_{\nu}$. This coefficient in turn is determined by the thermodynamic potentials of the system. The application of thermodynamics to EHD is legitimate, because the internal relaxation time of the e-h liquid is ${ }^{2.4} \lesssim 1$ nisec, much shorter than the lifetimes $\tau_{0}=40 \mu \mathrm{sec}$ for $\mathrm{Ge}^{2.5}$ and $\tau_{0} \approx 0.2 \mu \mathrm{sec}$ for Si. ${ }^{2.6}$ By solving the rate equations, Eqs. (2.1) and (2.2), we, find that $h_{\nu}$ is determined by an effective free energy difference $\psi_{\nu}$ which includes the effects of the EHD lifetime $\tau_{0}$. This free energy difference is analogous to the free energy $\Delta f_{v}$ required to create a 
drop of classical $\left(\tau_{0} \rightarrow \infty\right)$ 1iquid from $\nu$ vapor molecules discussed in chapter 1 , and can be written in the form $\psi_{v}=\Delta \mathrm{f}_{\nu}+\mathrm{f}_{\nu}{ }^{\tau}$, where $\mathrm{f}_{\nu}{ }^{\tau}$ is the contribution from $\tau_{0}$.

The total Helmholtz free energy $f_{v}$ of a freely moving drop of $v$ $\mathrm{e}-\mathrm{h}$ paifs is the sum of the translational, rotational, and internal contributions :

$$
\mathrm{f}_{\nu}=\mathrm{f}_{\nu}^{\operatorname{tran} s}+\mathrm{f}_{\nu}^{\text {rot }}+\mathrm{f}_{\nu}^{\text {int }}
$$

Assuming an ideal gas of rigidly rotating drops, we have

$$
\mathrm{f}_{v}^{\text {trans }}=\mathrm{kT} \ln \left(\mathrm{g}_{v} / \mathrm{eD}_{v}\right)
$$

where $D_{\nu} \equiv\left(\nu \mathrm{m} * \mathrm{kT} / 2 \pi \hbar^{2}\right)^{3 / 2}$ is the effective density of states for drops of mass $\nu m^{*}$; and

$$
\mathrm{f}_{V}^{\operatorname{rot}}=\mathrm{kT} \ln \left[(\pi / 8)^{1 / 2}\left(\hbar^{2} / \mathrm{IkT}\right)^{3 / 2}\right]
$$

where $I=(2 / 5) m * r_{0}^{2} v^{5 / 3}$ is the EHD moment of inertia. The interna1 Helmholtz free energy $f_{v}$ int is given by

$$
f_{\nu}^{\text {int }}=4 \pi x_{o}^{2} \sigma v^{2 / 3}-\nu \phi
$$

where $\sigma$ is the surface tension, and $\phi$ is the condensation energy of an e-h pair relative to $\mathrm{FE}$. The surface tension is approximately related to the surface energy $\mathrm{w}$ by $\sigma \simeq \mathrm{w}\left[1-\left(\mathrm{T} / \mathrm{T}_{\mathrm{C}}\right)^{2}\right]$, where $\mathrm{T}_{\mathrm{c}}$ is the critical temperature (see for example Ref. 2.7). Equations (2.3) to (2.6) are strictly valid only for macroscopic drops, but should be 
reasonably accurate for complexes of size greater than $v \sim 10$.

The chemical potential of an ideal $\mathrm{FE}$ gas of density $\mathrm{n}$ is given by

$$
\mu_{x}=k T \ln \left(n / D_{x}\right)
$$

where $D_{x} \equiv \gamma_{x}\left(m * k T / 2 \pi \hbar^{2}\right)^{3 / 2}$ is the effective density of states, and $\gamma_{x}$ is the degeneracy of the FE ground state. By equating $\mu_{x}$ with the condensation energy $-\phi$ we obtain the saturated $\mathrm{FE}$ density,

$$
\mathrm{n}_{\mathrm{s}}=\mathrm{D}_{\mathrm{x}} \exp (-\phi / k \mathrm{~T})
$$

Were it not for the existence of the surface tension $\sigma$ and the lifetime $\tau_{0}$, condensation would occur whenever the $F E$ density $n$ reaches $n_{s} \cdot$ The change in the bulk Helmholtz free energy of the FE-EHD system when an e-h pair is transferred from an EHD to a FE neglecting drop translation and rotation is $\mu_{x}+\phi=x k T$, where we define

$$
\mathrm{x} \equiv \ln \left(\mathrm{n} / \mathrm{n}_{\mathrm{S}}\right)
$$

the degree of supersaturation. It is this free energy difference which provides the driving force for the nucleation and growth of drops. The total internal free energy difference $\Delta \mathrm{f}_{\nu}$ is then given by

$$
\Delta \mathrm{f}_{\nu}=\mathrm{f}_{\nu}{ }^{\mathrm{int}}-\nu \mathrm{\mu}_{\mathrm{x}}=4 \pi \mathrm{r}_{\mathrm{o}}^{2} \sigma v^{2 / 3}-\mathrm{xkT} \nu
$$


the expression which enters into classical nucleation theory , as discussed in chapter 1 ,

With this information we can derive the evaporation coefficient $\alpha_{\nu}$ from Eq. (2.1) by using the principle of detailed balance. Because each droplet very rapidly establishes internal thermodynamic equilibrium, $\alpha v$ must be independent of the EHD lifetime. Therefore we can treat EHD as drops of classical fluid for the purpose of deriving $\alpha_{v}$ and ignore the last term in Eq. (2.1). In dynamic equilibrium $\left(\mathrm{dg}_{\nu} / \mathrm{dt}=0, \mathrm{~J}_{\nu}=0\right)$ we have

$$
d_{v-1} \beta n(\nu-1)^{2 / 3}=d_{\nu} \alpha_{v} v^{2 / 3}
$$

where we use $d_{v}$ to denote the classical $\left(\tau_{0} \rightarrow \infty\right)$ equilibrium size distribution. We can obtain an expression for $d_{v}$ by equating $\mu_{x}$ with $d\left(d_{v} f_{v}\right) / d d_{v}$, the chemical potential of the gas of drops of size $v$. We find

$$
\mathrm{d}_{v}=\mathrm{H}_{v} \exp \left(-\Delta \mathrm{f}_{v} / \mathrm{kT}\right)
$$

where $\Delta \mathrm{f}_{\nu}$ is given by $\mathrm{Eq} .(2.10)$, and the slow $\nu$ dependence of $\mathrm{f}_{\nu}^{\text {rot }}$ has been included in the new effective density of states $\mathrm{H}_{v} \equiv \mathrm{D}_{v} \exp \left(-\mathrm{f}_{v}{ }^{\operatorname{rot}} / \mathrm{kT}\right)$. By using Eq. (2.12) for $\mathrm{d}_{v}$ in Eq. (2.11), and by assuming $\nu \gg 1$, we find the evaporation coefficient,

$$
\alpha_{\nu}=\left(\mathrm{H}_{\nu-1} / \mathrm{H}_{\nu}\right) \beta \mathrm{n}_{\mathrm{s}} \exp \left(8 \pi \mathrm{r}_{\mathrm{o}}^{2} \sigma / 3 \nu^{1 / 3} \mathrm{kT}\right)
$$

where $\left(\mathrm{H}_{v-1} / \mathrm{H}_{\nu}\right) \cong 1$. The argument of the exponential in Eq. (2.13) is just the difference in surface free energy between a drop of size $v$ 
and one of size $\nu-1$ divided by $\mathrm{kT}$. Thus the surface tension increases the evaporation rate of small drops. For large drops $\alpha_{v}$ reduces to the Richardson expression $\mathrm{Bn}_{\mathrm{S}}$.

Returning to the original rate equations, Eqs. (2.1) and (2.2), we are now in a position to calculate the EHD size distribution in dynamic equilibrium $h_{\nu}$, including the effect of the EHD lifetime. Setting $d g_{v} / d t=0$ and $J_{v}=0$, Eqs. (2.1) and (2.2) reduce to the following recursion relation:

$$
h_{v} / h_{v-1}=\left(d_{v} / d_{v-1}\right) /\left(1+v^{1 / 3} / \alpha_{v} \tau_{o}\right)
$$

Because an EHD as formulated is indistinguishable from a FE for $\nu=1$, we have the boundary condition $h_{1}=d_{1}=n$. By using Eq. (2.14) repeatedly we find

$$
h_{\nu}=d_{\nu} \exp \left[-\sum_{j=2}^{v} \ln \left(1+\frac{j^{1 / 3}}{\alpha_{j}^{\tau}{ }_{0}}\right)\right] \text {. }
$$

From Eq. (2.12) for $d_{v}$ it is apparent we can put $h_{v}$ in a similar form by defining the quasi free energy $f_{v}{ }^{\tau}$ so that the contents of the square brackets in Eq. (2.15) are equal to $\mathrm{f}_{v}{ }^{\mathrm{T}} / \mathrm{kT}$. We have finally:

$$
h_{v}=H_{v} \exp \left(-\psi_{v} / k T\right)
$$

where the effective free energy difference $\psi_{v}$ is given by $\psi_{v}=\Delta f_{v}+f_{v}{ }^{\tau}$. Although the size distribution in dynamic equilibrium given by Eq. (2.16) is inapplicable above $\mathrm{T} \sim 1.3 \mathrm{~K}$, where the FE-EHD system is characterized 


\section{0 a 30793}

by metastable states ${ }^{2.8,2.9,2.10}$ (see chapter 5), it is useful in analyzing the actual process of nucleation.

\section{Free Energy Difference}

An understanding of the origins of metastability in the FE-EHD system is aided by detailed consideration of the potential $\psi_{V}$. Explicitly written out, it is

$$
\psi_{\nu}=4 \pi r_{o}{ }^{2} \sigma \nu^{2 / 3}-x k T \nu+k T \sum_{j=2}^{\nu} \ln \left(1+\frac{j^{1 / 3}}{\alpha_{j} \tau_{o}}\right) \text {. }
$$

The three terms in $\mathrm{Eq} .(2.17)$ are respectively contributions from surface, bulk, and lifetime effects. The free energy difference $\psi_{v}$ from Eq. (2.17) is plotted for Ge at $2 \mathrm{~K}$ in Figs. 2.1 and 2.2 for various values of the degree of supersaturation $x$. For small drop radii $R=r_{0} \nu^{1 / 3}$ the surface contribution always dominates $\psi_{\nu}$. If $\mathrm{x}$ is larger than a minimum value $\mathrm{x}_{\mathrm{m}}$, Figs. 2.1(a) and $2.2(\mathrm{a})$, the negative bulk free energy difference -xkT $\nu$ drives $\psi_{\nu}$ downward, creating a maximum $\psi_{c}$ at the critical radius $\mathrm{R}_{\mathrm{c}}$, indicated in $\mathrm{Fig}$. 2.1. For greater radii the positive lifetime term $\mathrm{f}_{v}{ }^{\top}$ becomes large, creating a very sharp minimum $\psi_{S}$ at the stable radius $R_{S}$. Although $R_{C}, R_{S}, \psi_{c}$, and $\psi_{S}$ must in general be calculated numerically from Eq. (2.17), the following expressions are valid if $R_{S} \gg R_{c}$ :

$$
\begin{aligned}
& \mathrm{R}_{\mathrm{c}} \simeq 2 \sigma / \mathrm{n}_{\mathrm{o}} \mathrm{xkT} ; \\
& \mathrm{R}_{\mathrm{S}} \simeq \mathrm{r}_{\mathrm{o}} \beta \tau_{\mathrm{o}}\left(\mathrm{n}-\mathrm{n}_{\mathrm{s}}\right) ;
\end{aligned}
$$




$$
\psi_{c} \simeq\left[16 \pi \sigma^{3} / 3\left(n_{o} x k T\right)^{2}\right]
$$

Here Eqs. (2.18) and (2.20) are identical to the expressions obtained in classical nucleation theory,,$^{2.2,2.11}$ and Eq. (2.19) is the same as the result found from the EHD rate equations developed by Pokrovskii. 2.6

As shown in Figs. 2.1 and 2.2, there exist two wells in $\psi_{V}$ : one about $\nu=1$ populated by $F E$ and multiexciton (ME) complexes, and one about $\mathrm{R}_{\mathrm{s}}$ populated by $\mathrm{m}$ acroscopic drops. Within each we11 local equilibrium is rapidly established, and the density of ME of less than critical size is $\mathrm{g} i$ ven by the equilibrium expression Eq. (2.16). If this expression were also valid for macroscopic drops at $2 \mathrm{~K}$, the "equilibrium" threshold $\mathrm{x}_{\mathrm{e}}=0.771$, Figs. 2.1(c) and 2.2(c), would be observed when the concentration of drops rapidly became significant. However, the drop well is isolated from free and multiexcitons by the large potential barrier $\psi_{c}$; as a result the concentration of drops in the well is not fixed by Eq. (2.16) but depends upon the history of the system. The degree of supersaturation must be increased to the "up-going" threshold value $\mathrm{x}_{+}=2.01$ (or $\left.\mathrm{n} / \mathrm{n}_{\mathrm{s}}=7.46\right)$, Figs. 2.1(e) and 2.2(e), before $\psi_{c}$ is small enough to permit the nucleation of new drops. Similarly, x must be reduced to the "down-going" threshold $x_{-}=0.734$ (or $n / n_{s}=2.08$ ), Fig. 2.1(b), before drops already present can "escape" over the potential barrier and break up into FE. The appropriate formation and breakup "currents", $J_{+}$and $J_{-}$, are indicated in Fig. 2.1. As we show in the next section, both thresholds are very sharp. 


\section{$00 \div 40,4794$}

As also shown in Figs. 2.1 and 2.2, the well about $\mathrm{R}_{\mathrm{s}} \mathrm{d}$ isappears for values of $x$ less than the minimum value $x_{m}=0.728$ (or $n / n_{s}=2.07$ ) defined by the condition that the first and second derivatives of $\psi_{v}$ from Eq. 2.17 are simultaneously zero. Consequently, macroscopic drops cannot exist in $\mathrm{Ge}$ at $2 \mathrm{~K}$ for supersaturation ratios less than $\mathrm{n} / \mathrm{n}_{\mathrm{s}}=2.07$, or with stable radii less than the value at $\mathrm{x}_{\mathrm{m}}, \mathrm{R}_{\mathrm{m}} \simeq 0.19 \mu \mathrm{m}$ from Fig. 2.1(a). These effects follow from the inclusion of the EHD lifetime in Eqs. (2.1) and (2.2) and were first reported by Silver 2.12 However, his prediction that actual drop radii in Ge above $1.3 \mathrm{~K}$ are determined by the value of $R_{s}$ at the equilibrium threshold $x_{e}$ cannot be accurate.

When the temperature-independent evaporation rate $c v^{2 / 3}$ is included in the calculation of $h_{\nu}$, the same result is obtained except $f_{\nu}{ }^{\top}$ from Eq. (2.15) is replaced by the expression

$$
f_{\nu}{ }^{\tau}=k T \sum_{j=2}^{\nu} \ln \left(1+\frac{j^{1 / 3}+c \tau_{o}}{\alpha_{j} \tau_{o}}\right),
$$

which also replaces the last term in Eq. (2.17) for $\psi_{\nu^{*}}$ From Eq. (2.21), we find that the effect of the coefficient $c$ is to increase the size of the lifetime term in $\psi_{\nu}$, and therefore to increase the expected deviations from classical nucleation theory (see chapter 3).

A point we have previously neglected is that the lifetimes $\tau_{\nu}$ of sma11 ME complexes $(\nu \lesssim 10)$ certainly differ from the bulk value $\tau_{0}$. If these lifetimes were known, we could use them in Eq. (2.17), where an unusua11y short $\tau_{\nu}$ causes a positive step in $f_{\nu}{ }^{\top}$ and $\psi_{\nu}$. As long 
as $\alpha_{v} \tau_{v}>1$ (or $\tau_{v} \gtrsim 30 \mathrm{nsec}$ for Ge at $2 \mathrm{~K}$ ), the potential $\psi_{v}$, and therefore $h_{v}$ and the formation rate $J_{+}$, are insensitive to the values of $\tau_{\nu}$. However if $\alpha_{\nu} \tau_{\nu} \ll 1$ for any small value of $\nu$, then many body collisions are required to bridge the gap between long-1ived sizes, lowering the distribution $h_{\nu}$ for larger $v$, and decreasing the formation rate $J_{+} \cdot$ Bearing this possible complication in mind, we shall use $\tau_{v}=\tau_{0}$ for the remainder of this paper.

\section{Calculation of Nucleation Rates}

With knowledge of the dynamic equilibrium size distribution $h_{v}$ and the potential $\psi_{\nu}$, we can now calculate the rates of formation $\mathrm{J}_{+}$ and breakup $J_{-}$of macroscopic drops in the steady state $\left(\mathrm{dg}_{V} / \mathrm{dt}=0\right)$. Returning to the rate equations, Eqs. (2.1) and (2.2), we divide through by $h_{v-1}$ and use Eq. (2.13) to obtain

$$
g_{v-1} / h_{v-1}-g_{v} / h_{v}=J / h_{v-1} \beta n(v-1)^{2 / 3}
$$

where $J=J_{v}$ is the size-independent net formation rate in the steady state. Of course $\mathrm{J}$ cannot truly be positive for arbitrarily large $v$; mathematical solutions of $\mathrm{Eq} \cdot(2.22)$ for $g_{V}$ pass through zero at a finite drop size and become unphysically negative beyond. It is interesting to note that Eq. (2.22) is a diffusion equation for the function $g_{v} / h_{v}$ with the diffusion coefficient $h_{v-1} \beta n(\nu-1)^{2 / 3}$. The boundary conditions for this difference equation are appropriately made at the $\mathrm{FE}$ and stable sizes $\nu=1$ and $\nu_{s}$. Summing Eq. (2.22) 
between $v=2$ and $v_{S}$ and approximating the sum by an integral we have

$$
\left(g_{1} / h_{1}\right)-\left(g_{s} / h_{s}\right)=(J / \beta n) \int_{1}^{\nu} d v\left(h_{\nu} \nu^{2 / 3}\right)^{-1} .
$$

For the formation rate $\mathrm{J}_{+}$the appropriate boundary conditions are $\mathrm{g}_{1} / \mathrm{h}_{1}=1$ and $\mathrm{g}_{\mathrm{S}} / \mathrm{h}_{\mathrm{s}}=0$, i.e. no macroscopic drops are initially present. We define the breakup rate $J_{-}$by the boundary conditions $g_{1} / h_{1}=0$ and $g_{S} / h_{S}=N / h_{S} \Delta \nu_{s}$, where $N$ is the actual non-equilibrium concentration of drops in the well about $\nu_{s}$, and $\Delta \nu_{s} \equiv\left[\left(\mathrm{d}^{2} \psi_{\nu} / \mathrm{d} \nu^{2}\right)_{\nu_{s}} / 2 \nu \mathrm{kT}\right]^{-1 / 2}$ is the Gaussian width of the well. With this definition the net formation rate is given by $J=J_{+}-J_{-}$. Because $1 / h_{\nu}$ has a very sharp maximum at $\nu=\nu_{c}$ (see Figs. 2.1 and 2.2), we can perform the integral in Eq. (2.23) by expanding $\psi_{v}$ about $v_{c}$ to second order, setting $\nu=v_{c}$ outside the exponential, and extending the limits of integration to $\pm \infty$. In this way we obtain the final results:

$$
\begin{aligned}
& \mathrm{J}_{+}=\beta n \nu_{c}^{2 / 3} \mathrm{~h}_{\mathrm{c}} / \Delta \nu_{\mathrm{c}} ; \\
& \mathrm{J}_{-}=\mathrm{J}_{+} \mathrm{N} / \mathrm{h}_{\mathrm{s}} \Delta \nu_{\mathrm{s}} .
\end{aligned}
$$

The subscripts $\mathrm{c}$ and $\mathrm{s}$ refer to the critical and stable sizes $\nu_{c}$ and $\nu_{s}$, and $\Delta \nu_{c}$ is defined in the same way as $\Delta \nu_{s}$. The form of Eq. (2.24) for $\mathrm{J}_{+}$can be easily understood as the product of the $\mathrm{FE}$ collision rate $B n \nu_{c}^{2 / 3}$ with the concentration gradient $h_{c} / \Delta \nu_{c}$ at the critical size driving the diffusion of droplets over the potential barrier $\psi_{c}$ to larger sizes. The expression $\mathrm{Eq} \cdot(2.25)$ for $\mathrm{J}_{-}$is similar except the 
breakup rate is weighted by the ratio of the actual concentration of drops $N$ to the concentration $h_{S} \Delta \nu_{S}$ in dynamic equilibrium. Therefore the potential barrier to be surmounted is the difference $\psi_{c}-\psi_{S}$ between $\psi_{\nu}$ at the critical and stable sizes, rather than $\psi_{c}$ (see Figs. 2.1 and 2.2). In order for these solutions, Eqs. (2.24) and (2.25) to be valid when the $F E$ density changes with time, it is only necessary that the size distribution have the steady-state value near the critical radius. For the formation rate the time required for this to happen is roughly $\nu_{\mathrm{c}}^{1 / 3} / \beta \mathrm{n}$, which is $\lesssim 1 \mu \mathrm{sec}$ for $\mathrm{Ge}$ at $2 \mathrm{~K}$. For $\mathrm{J}_{-}$ this time is roughly $\nu_{S}^{1 / 3} / \beta n \sim \tau_{0}$, which is $\simeq 40 \mu \mathrm{sec}$ for $\mathrm{Ge}$. Therefore these solutions can be applied to situations where the rise and fall times of the excitation are moderately fast.

Semilogarithmic plots of $\mathrm{J}_{+}$and $\mathrm{J}_{-}$, computed from Eqs (2.24) and (2.25), versus $x$, the degree of supersaturation, are shown for $G e$ in Fig. 2.3. The most striking feature of these graphs is the exceptionaliy strong dependence of $\mathrm{J}_{+}$and $\mathrm{J}_{-}$upon the degree of supersaturation. As $x$ increases at $2 \mathrm{~K}$ from 1.8 to $2.2, J_{+}$increases by 9 orders of magnitude from $10^{-3}$ to $10^{6}$ drops $\mathrm{cm}^{-3} \mathrm{sec}^{-1}$. Similarly J_increases at $2 \mathrm{~K}$ by 14 orders of magnitude, from $10^{-4}$ to $10^{10} \mathrm{drops} \mathrm{cm}^{-3} \mathrm{sec}^{-1}$, for a decrease in $\mathrm{x}$ of only 0.007 . At $1.5 \mathrm{~K}$ and $1.25 \mathrm{~K}$ the variation of these currents with $\mathrm{x}$, shown in Fig. 2.3, is somewhat slower but still extremely steep. The variation of the currents $J_{+}$and $J_{-}$with $x$ is so rapid that we can speak of thresholds $x_{+}$and $x_{-}$at which $J_{+}$and $J_{-}$ respectively "turn on". The same mathematical phenomenon occurs in classical nucleation theory. Workers in cloud physics have defined 


\section{0,0494796

the threshold $x_{+}$as that value of $x_{\text {for }}$ which the minimum detectable concentration of drops $\mathrm{N}_{\min }$ is formed in the observation time $\Delta t$,

$$
\mathrm{J}_{+} \Delta \mathrm{t}=\mathrm{N}_{\min }
$$

Typically $\mathrm{J}_{+} \sim 10^{2}$ drops $\mathrm{cm}^{-3} \mathrm{sec}^{-1}$ at threshold for sensitive luminescence detection of EHD in Ge. The threshold $x_{-}$can be defined in the same way. In chapter 3 we give precise definitions of $x_{+}$and $x_{-}$ which do not depend upon experimental sensitivity. However, this improvement is primarily conceptual; the actual values of $x_{+}$and $x_{-}$ obtained by the two methods do not significantly differ.

A second major feature of the behavior of the FE-EHD system illustrated by Fig. 2.3 is that both the rates of formation and breakup are negligible at $1.5 \mathrm{~K}$ and $2 \mathrm{~K}$ for a range of values of $\mathrm{x}$ between the thresholds $x_{-}$and $x_{+}$. If the $\mathrm{FE}$ density lies in this range, the concentration of drops $\mathrm{N}$ cannot change appreciably in a physically reasonable time and is determined only by the history of excitation. A rough estimate of the time required to reach dynamic equilibrium $\left(\mathrm{J}_{+}=\mathrm{J}_{-}\right)$is given by the magnitude of $\mathrm{J}_{+}$and $\mathrm{J}_{-}$where they intersect. At $1.5 \mathrm{~K}$ the curves meet at $J_{+}=J_{-} \simeq 10^{-6} \mathrm{sec}^{-1} \mathrm{~cm}^{-3}$, implying an equilibration time $\sim 10^{6} \mathrm{sec}$; this time becomes astronomically 1 ong at $2 \mathrm{~K}$. Therefore any practical experiment performed on Ge at these temperatures can only observe metastable states of the system. These effects provide an explanation of the hysteresis observed $2.8,2.9,2.10$ in the luminescence of EHD in Ge. This hysteresis was found to 
persist for times longer than $4000 \mathrm{sec},^{2.9}$ (see chapter 5) indicating that individual drops in the samples used survived for comparable times. In general a number of mechanisms may be present which destroy drops, for example inhomogeneous strains and electrical contacts. If the 1ifetime of drops against destruction is very short, the observed state of the system is determined by these externa1 mechanisms. In either case, equilibrium arguments are inapplicable to the FE-EHD system in Ge above $\mathrm{T} \sim 1.3 \mathrm{~K}$.

However, at temperatures $\mathrm{T} \lesssim 1.3 \mathrm{~K}$ the situation changes. As shown in Fig. 2.3, the separation $x_{+}-x_{-}$of the thresholds decreases as the temperature is reduced below $2 \mathrm{~K}$; at $1.25 \mathrm{~K}$ the thresholds cross. At this temperature drops created at the formation threshold $x_{+}=5.6$ in Fig. 2.3, are immediately destroyed by the large breakup rate J_. In order to stably create drops, the degree of supersaturation must be increased to the equilibrium threshold $\mathrm{x}_{\mathrm{e}}=5.8$ where $\mathrm{J}_{+}$and $\mathrm{J}_{-}$ are equal and the system is in dynamic equilibrium. This threshold can be defined by the condition that the population $h_{s} \Delta \nu_{s}$ of the droplet well in $\psi_{\nu}$ is equal to the minimum detectable drop concentration $\mathrm{N}_{\text {min }}$. The physical reason metastability disappears in Ge below $\mathrm{T} \sim 1.3 \mathrm{~K}$, is that the $\mathrm{FE}$ density near the saturated value $\mathrm{n}_{\mathrm{S}}$ is far too small to support the volume decay of EHD of finite size. Equivalently, the evaporation coefficient $\alpha_{v} \simeq \beta_{\mathrm{S}}$ becomes very small; when $\alpha_{\nu} \tau_{0}<1$, the lifetime term in Eq. (2.17) becomes large at the critical size and dominates $\psi_{c}$, the potential barrier to nucleation. Once the degree of supersaturation $\mathrm{x}$ has been increased to a value sufficient to create 


\section{7}

a drop well in $\psi_{v}$, the potential barrier $\psi_{c}$ is too small to impede condensation, so that drops form as soon as $\mathrm{x}$ exceeds the minimum value $x_{m}$ (see Figs. 2.1(a) and 2.2(a)). In chapter 3 we consider the detailed temperature dependence of the FE density thresholds.

\section{Inhomogeneous Nucleation}

The problem of calculating the rates of drop formation $\mathrm{J}_{+}$and breakup $J_{-}$, and the size distribution $h_{v}$ in dynamic equilibrium for EHD nucleated on condensation centers is quite similar to that for homogeneous nucleation. In this section we describe only the differences in the two calculations and summarize the results.

The FE-EHD system we consider is identical to that of section 22 except that ME complexes and drops of all sizes $\nu>1$ are bound to nucleation centers and are therefore no longer free to move. Consequently the total Helmholtz free energy $f_{\nu}$ of a drop becomes

$$
\mathrm{f}_{v}=\mathrm{f}_{v}^{\text {int }}-\mathrm{B}_{v}
$$

where the internal free energy $f_{v}$ int is given by Eq. (2.6), and $B_{v}$ is the binding energy of the drop on the center. Because the density $N_{i}$ of nucleation centers is assumed to be fixed, the "classical" $\left(\tau_{0} \rightarrow \infty\right)$ probability that a drop of size $\nu$ is present on a given center is proportional to $\exp \left[-\left(f_{v}-\nu \mu_{x}\right) / k T\right]$. This expression replaces $d_{v}$ in the calculation of the evaporation coefficient $\alpha_{v^{*}}$ Carrying out the calculation of the size distribution in dynamic equilibrium, we find: 


$$
\begin{aligned}
& \mathrm{h}_{v}=\left(\mathrm{N}_{i} / \mathrm{Z}\right) \exp \left[-\left(\psi_{v}-\mathrm{B}_{v}\right) / \mathrm{kT}\right] ; \\
& \mathrm{Z} \equiv \sum_{\nu=0}^{\infty} \exp \left[-\left(\psi_{v}-\mathrm{B}_{v}\right) / \mathrm{kT}\right] .
\end{aligned}
$$

Here $\psi_{\nu}$ is given by Eq. (2.17) as before, and $z$ is the analog of the partition function. An equation similar to Eq. (2.28) has also been obtained by Silver. ${ }^{2.12}$ Comparing the homogeneous and inhomogeneous expressions for $h_{\nu}$, Eqs. (2.16) and (2.28), we find that the binding energy $B_{V}$ increases Eq. (2.28) by the factor $\exp \left(B_{v} / k T\right)$, but that the lower density of states decreases Eq. (2.28) by the factor $\left(\mathrm{N}_{\mathrm{i}} / \mathrm{ZH}_{V}\right)$. The function $Z$ normalizes $h_{v}$ so that the total density of drop "sites" is equal to $\mathrm{N}_{i}$. If the potential $\psi_{v}-\mathrm{B}_{\nu}$ possesses a negative minimum at a size $v_{M}$ less than the critical value $v_{c}$ the size distribution saturates $\left(h_{M} \simeq N_{i}\right)$ and $Z$ is given approximately by $1 n z \simeq-\left(\psi_{M}-B_{M}\right) / k T$, where the subscript $M$ refers to the size $\nu_{M}$.

The calculation for the rates of formation $J_{+}$and breakup $J_{-}$of EHD bound to nucleation centers is formally identical to the homogeneous case. In order to obtain final results, we need only substitute $h_{v}$ from Eq. (2.28) into Eq. (2.24) for $\mathrm{J}_{+}$and into Eq. (2.25) for $\mathrm{J}_{-}$. The qualitative features of the behavior of $\mathrm{J}_{+}$and $\mathrm{J}_{-}$, illustrated by Fig. 2.3, remain unchanged; the on1y major effect of nucleation centers is to shift the positions of the thresholds, $\mathrm{x}_{+}$and $x_{-}$, for the creation and destruction of drops. Contrary to what one might expect, these shifts are not necessarily large. We consider the detailed behavior of the thresholds $x_{+}$and $x_{-}$in chapter 3 . 
of the various types of crystal imperfections, neutral impurities are probably most important as nucleation centers; experimentally we find dislocations are not effective, ${ }^{2.9}$ (see chapter 5) and vacancy clusters and deep traps can be practically eliminated by crystal growing techniques. The binding energy $B_{v}$ of an EHD on a neutra1 impurity varies in general with $\nu$, but should have the size independent value $B$ whenever the drop radius exceeds the screening length of the plasma; a reasonable estimate of the smallest size at which this occurs is $\nu \sim 10$. Because the critical size for Ge at $2 \mathrm{~K}$ is $\nu_{\mathrm{c}} \gtrsim 50$ from Fig. 1(e), we can ignore the possible shift in $\nu_{c}$ caused by the size dependence of $B{ }^{\cdot}$ As a result the breakup rate $J_{-}$is independent of the value of $B$ and differs from the homogeneous expression only by the factor $\mathrm{H}_{\mathrm{S}} / \mathrm{H}_{\mathrm{C}}$. A convenient parameter which determines the effect of impurities upon the formation rate $J_{+}$is the barrier lowering $\Delta \psi$, which we define by $\Delta \psi \equiv \mathrm{B}-\mathrm{kT} \ln \mathrm{Z}$.

Possibly the most important role of nucleation centers is to stabilize small multiexciton complexes. To date there is no experimenta1 proof that free $\mathrm{ME}$ complexes exist in $\mathrm{Ge}$ or $\mathrm{Si}$. If in fact any complexes of size $\nu \lesssim 10$ are not bound, many body collisions are required to bridge the gap in stable sizes and the homogeneous formation rate $J_{+}$ is greatly reduced. In this case EHD nucleation would occur first on centers regardless of the value of $B$.

Finally, we consider the effect of a possible electrical charge on EHD upon the formation rate, either for homogeneous or inhomogeneous nucleation. For classical fluids such as water, the electrostatic 
self energy $E_{c}=e^{2} / 2 R_{c}$ of a singly charged critical drop is comparable to the potential barrier $\Delta f_{c}$ for vapor densities near the uncharged formation threshold. It is a straightforward algebraic exercise to show that if $\mathrm{E}_{\mathrm{c}} / \psi_{\mathrm{c}}>2(3 / 4)^{4} \simeq 0.63$, the total drop free energy $\Delta \mathrm{f}_{\nu}+\mathrm{e}^{2} / 2 \mathrm{R}$ decreases monotonically with $R$, and the potential barrier to nucleation is destroyed. Consequent1y, the presence of ions in the vapor greatly reduces the degree of supersaturation at which condensation occurs. However, for Ge at $\mathrm{T}=2 \mathrm{~K}$ this self energy is $\mathrm{E}_{\mathrm{c}}=\mathrm{e}^{2} / 2 \varepsilon \mathrm{R}_{\mathrm{c}} \lesssim 1.1 \mathrm{meV}$ where $\varepsilon=16$ is the dielectric constant, and $R_{c} \geq 0.04 \mu \mathrm{m}$ from Fig. 2.1. This value of $E_{c}$ is much smaller than the potential barrier $\psi_{c} \gtrsim 10 \mathrm{meV}$ and does not greatly alter the total drop free energy for smaller sizes. Thus we conclude that the presence of a single charge on an EHD at temperatures $\mathrm{T} \gtrsim 2 \mathrm{~K}$ has only a small effect upon the position of the formation threshold. At lower temperatures $\mathrm{T} \sim 1.5 \mathrm{~K}$, where the values of $\mathrm{R}_{\mathrm{c}}$ and $\psi_{c}$ at the formation threshold are reduced, the effect of a single drop charge is somewhat larger, but still small compared to that of an impurity atom. Why are not embryos doubly charged? The answer is that the self energy of a doubly charged drop smaller than the critical size is $\gtrsim 5 \mathrm{meV}$, larger than the binding energy $\cong 3 \mathrm{meV}$ of a carrier in an EHD. Accounting for the existence of image charges, we conclude that an embryo which becomes doubly charged will very rapidly rid itself of the excess charge by the emission of a free carrier in a time of the order $R / v_{F}<10^{-11} \mathrm{sec}$, where $v_{F}$ is the Fermi velocity. Such an embryo does not exist long enough to grow larger and influence the process of nucleation. 


\section{9

CHAPTER 3. APPLICATION OF NUCLEATION THEORY TO OBSERVABLE PHENOMENA

In chapter 2 we calculated the non-equilibrium rates of formation $\mathrm{J}_{+}$and breakup $\mathrm{J}_{-}$of macroscopic electron-hole drops (EHD) in a supersaturated free-exciton (FE) gas. We also calculated the distribution $h_{\nu}\left(\right.$ drops $\left.\mathrm{cm}^{-3}\right)$ of EHD versus drop "size" $\nu$ (e-h pairs) in dynamic equilibrium. We found that the FE-EHD system is characterized by metastable states at temperatures above $\mathrm{T} \sim 1.3 \mathrm{~K}$ in $\mathrm{Ge}$, and that the expressions for the currents $\mathrm{J}_{+}$and $\mathrm{J}_{-}$, and for the size distribution $h_{v}$, yield sharp thresholds in FE density.

In this chapter we use these expressions to predict quantitatively a number of experimentally observable phenomena in Ge assuming both homogeneous and inhomogeneous nucleation: Section 32, metastable time behavior; section 33, equilibrium and non-equilibrium thresholds in FE density, and hysteresis in EHD luminescence intensity; section 34 , procedure for accurately determining the EHD surface tension from threshold data; section 35, non-equilibrium FE-EHD coexistence curves which can be used to extract the EHD condensation energy $\phi$; section 36 ; drop radius near threshold versus temperature. Coexistence curves for Si are also presented in section 35. These calculations are made between $1 \mathrm{~K}$ and $4 \mathrm{~K}$ for $\mathrm{Ge}$, and between $3 \mathrm{~K}$ and $15 \mathrm{~K}$ for $\mathrm{Si}$, and they assume spatial uniformity in the FE gas. The contents of this chapter have been previously published, ${ }^{3.1}$ with the exception of the effect of temperature-independent evaporation on the EHD-FE phase diagram. 
A list of the parameters used in this calculation is given in Table 3.1. The value for Ge of $\phi$ is that of Lo, ${ }^{3.4}$ corrected for the temperature dependence reported by Thomas, Rice, and Hense1. ${ }^{3.5}$ This value of $\phi$ at $T=0$ agrees very well with the recently measured spectroscopic value ${ }^{3.9} \phi=(20.9 \pm 2.3) \mathrm{K}$, and the threshold measurement $\phi=(22.0 \pm 2.3) \mathrm{K}$ described in chapter 5 of this thesis. The value of $\mathrm{n}_{\mathrm{o}}$ for $\mathrm{Ge}$ in Table 3.1 also agrees very well with the recent measurement $\mathrm{n}^{3.9} \mathrm{n}_{\mathrm{o}}\left(10^{17} \mathrm{~cm}^{-3}\right)=(2.38 \pm 0.05)-(0.017 \pm 0.002) \mathrm{T}^{2}$.

For $\mathrm{Si}$ the parameters $\phi$ and $\mathrm{n}_{\mathrm{o}}$ were less we11 known at the time of the calculation. However the values given in Table 3.1 agree reasonably well with the more reliable recent measurements 3.10 $\phi(\mathrm{K})=(95 \pm 1)+(0.068 \pm 0.012) \mathrm{T}^{2}$ and $\mathrm{n}_{\mathrm{o}}\left(10^{17} \mathrm{~cm}^{-3}\right)=(33.3 \pm 0.5)-$ $(0.015 \pm 0.010) \mathrm{T}^{2}$. For $\mathrm{Si}$ we estimated $\sigma$ from the rough expression $\sigma \sim \frac{1}{2} \phi \mathrm{n}_{\mathrm{o}} \mathrm{r}_{\mathrm{o}}$, where the surface thickness was assumed to be equal to the Wigner-Seitz radius $r_{0} \equiv\left(3 / 4 \pi n_{0}\right)^{1 / 3} ;$ this estimate $\sigma \approx 8 \times 10^{-3} \mathrm{erg}^{-2}$ agrees very well with the recently calculated ${ }^{3.11}$ value $\sigma=8.75 \times 10^{-3} \mathrm{erg} \mathrm{cm}^{-2}$. The value given for $\sigma$ in Ge agrees with the measurement $\sigma=(2.6 \pm 0.3) \times 10^{-4} \mathrm{erg} \mathrm{cm}^{-2}$ at $\mathrm{T}=0$ described in chapter 5. The FE effective mass $\mathrm{m}^{*}$ and degeneracy for both $\mathrm{Ge}$ and Si are those of the lower ground state. ${ }^{3.7}$ The values of $N_{i}, G_{M}$, and $\Delta t$ (see below) are representative of actual experiments on ultrapure Ge and Si. Guided by experimental work on bound multiexciton (ME) complexes, $3.12,3.13$ we chose the temperature independent values 1isted for $\Delta \psi$, the barrier lowering due to impurities (see chapter 2). Assuming $\Delta \psi \approx \mathrm{B}$, the binding energy of a macroscopic EHD to a neutral 
sha1low impurity, the value $\mathrm{B} \approx 50 \mathrm{~K}$ for $\mathrm{Ge}$ in $\mathrm{Table} 3.1$ agrees reasonab1y we11 with the recent calculations for donors $3.14,3.15$ $B \approx 60$ to $120 \mathrm{~K}$. Except for the calculations shown in Fig. 3.4 below, the possible temperature-independent evaporation rate discussed in chapter 2 is assumed to be zero.

\section{:31 Conservation Equation}

In order to relate the results of chapter 2 to observable phenomena, we must specify the conditions under which a hypothetical experiment is performed. In the following calculations we assume that the source of excitation is uninterrupted, and that the generation rate $G$ $\left(\mathrm{cm}^{-3} \mathrm{sec}^{-1}\right)$ of $\mathrm{e}^{-\mathrm{h}}$ pairs is uniform within the effective volume occupied by FE. In the steady state the FE and drop concentrations, $\mathrm{n}$ and $\mathrm{N}$, are related to $\mathrm{G}$ by the conservation equation. Ignoring the rate of decay of free carriers we have

$$
\mathrm{G}=\mathrm{n} / \tau_{\mathrm{x}}+\mathrm{N} \nu / \tau_{\mathrm{o}}
$$

where $\tau_{x}$ and $\tau_{0}$ are the total $F E$ and EHD lifetimes. In this chapter $\nu$ refers to the stable drop size $\left(\nu_{S}\right.$ in chapter 2$)$ unless noted. Because the drop concentration $\mathrm{N}$ depends upon previous values of $\mathrm{G}$, we must specify the history of excitation. We consider two cases easily achieved experimentally: ${ }^{3.2,3.3}$ "up-going" data points taken by increasing $G$ from zero to a constant value $G_{U}$, and "down-going" points taken by first briefly increasing $G$ from zero to a fixed large value $G_{M}$, then decreasing $G$ to a constant intermediate level $G_{D}$. To 
simplify the analysis we assume that all changes in $G$ are made slowly compared to the lifetimes $\tau_{0}$ and $\tau_{x}$, so that the conservation equation, Eq. (3.1), holds at a11 times.

A qualitative description of the metastable behavior of $\mathrm{n}, \mathrm{N}$, and $\nu$ for up-going and down-going excitation is useful in understanding the calculations to follow. In the up-going case the $\mathrm{FE}$ density $\mathrm{n}$ increases with the generation rate until the up-going threshold $G_{+}=n_{+} / \tau_{x}$ is reached (chapter 2). The FE density is held near the threshold value $n_{+}$by the very rapid increase in the EHD formation rate $J_{+}$. Because the stable drop size $\nu$ depends only on $n$, the size $v_{+}$of drops produced is nearly constant and independent of the generation rate if the rate of increase of $G$ is sufficiently slow. The condition to be met is that the time $\sim \tau_{0}$ required for embryos to grow to the stable size is small compared to the average time between embryo formation $\sim\left(\mathrm{J}_{+} \mathrm{L}^{3}\right)^{-1}$ where $\mathrm{L}$ is the $\mathrm{FE}$ diffusion length. Because the rate of change of the generation rate above threshold is roughly $\mathrm{dG} / \mathrm{dt} \sim \mathrm{J}_{+} \nu_{+} / \tau_{0}$, this is true only if $\mathrm{dG} / \mathrm{dt} \lesssim\left(\nu_{+} / \mathrm{L}^{3} \tau_{0}^{2}\right)$. The corresponding lower limit on the risetime of $G$ to twice the threshold value in Ge (see sections 35 and 36 ) is quite long, $\sim 0.04 \mathrm{sec}$ at $2 \mathrm{~K}$ and $\sim 5 \mathrm{sec}$ at $1.5 \mathrm{~K}$. Calculations of $N, v$, and $n$ for fast pulsed excitation performed by Staehli. ${ }^{3.16}$ indicate that these quantities depend in general upon the risetime as well as the amplitude of the pulse. Once $G$ reaches the final constant value $G_{U}$, the EHD formation rate $J_{+}$is turned off by the reduction in the $\mathrm{FE}$ density through the conservation equation. The final concentration of drops is given approximately by 


\section{$00144904 \% 01$

$$
\mathrm{NV}+=\left(G_{U}-G_{+}\right) T_{0},
$$

where $v_{+}$depends only on the temperature.

In the down-going case the concentration $\mathrm{N}_{M}$ of drops of size $\nu_{+}$ is produced by the initially large generation rate $G_{M}$ in the manner described above. As $G$ decreases below $G_{M}$, the $E H D$ concentration must remain constant, because both the rates of formation and breakup are negligible (chapter 2). Therefore the FE density and the EHD size $v$ fall with $G$ until the down-going FE density threshold $n_{-}$is reached. As above, the $\mathrm{FE}$ density and $\mathrm{EHD}$ size remain near their down-going values $\mathrm{n}_{-}$and $\nu_{-}$as drops break up into free excitons. Once the final value $G_{D}$ has been reached, the breakup of EHD is halted by the resultant increase in FE density. For values of $G_{D}$ such that $n \simeq n_{-}$, the final concentration of drops is approximately given by

$$
N \nu_{-}=\left(G_{D}-G_{-}\right) \tau_{0},
$$

where $\nu_{-}$is also a function only of the temperature, and $G_{-}=n_{-} / \tau_{x}$ is the down-going threshold.

Before proceeding we consider the fact that the $\mathrm{FE}$ density is not spatially uniform in most experiments. Our expressions for the rates of formation and breakup, $J_{+}$and $J_{-}$, remain locally valid in cases where $\mathrm{n}$ changes slowly compared to the $\mathrm{FE}$ mean free path; condensation occurs first where $\mathrm{n}$ is largest. For volume excitation, the generation rate varies smoothly over distances $\sim 1 \mathrm{~mm}$ (see chapter 4 ) and thus the model is directly applicable; volume excitation was used to obtain the 
central results reported in chapter 5 of this thesis. For relatively low levels of surface excitation, the drop bearing volume is sma11 compared to the extent of the $\mathrm{FE}$ gas, and the spatial form of $\mathrm{n}(\mathrm{x})$ is fixed; the FE density is everywhere proportional to the largest value near the excited surface. Consequent1y, a spatially uniform model can be used to describe the properties of EHD near threshold for both volume and surface excitation. Using much higher levels of optical excitation focussed to a sma11 spot, several authors $3.17,3.18$ have reported phenomena involving the transport and destruction of EHD. Near threshold for unfocussed optical excltation, drops probably remain near the source of excitons, pinned to impurities either during or after nucleation. The fact that the observed hysteresis in the luminescence of EHD in Ge persists for periods of time longer than 1 hour $^{3.2}$ (see chapter 5) provides strong evidence that single drops can exist for comparable times.

\section{Metastab1e Time Development}

In this section we obtain approximate solutions for the very slow time dependence of the EHD density following up-going and down-going steps in the generation rate. By doing so, we can explain the observed $^{3.2}$ (see chapter 5) very slow drift of the EHD luminescence intensity. In section 33 we use these solutions to define precisely the thresholds $n_{+}$and $n_{-}$.

In the up-going case we start with the relation $\mathrm{dN} / \mathrm{dt}=\mathrm{J}_{+} \cdot$ For the formation rate we use the approximate expression 


$$
\mathrm{J}_{+} \simeq \mathrm{C}_{+} \exp \left(\mathrm{n} \tau_{\mathrm{o}} / \mathrm{M}_{+} \tau_{\mathrm{x}}\right)
$$

suggested by Fig. 2.3 in chapter 2. The coefficients $\mathrm{M}_{+}$and $\mathrm{C}_{+}$are determined by expanding the exact expression for $J_{+}$about the threshold value (see section 33). In this way we find $M_{+}=n_{+} \tau_{0} / \nu_{c}{ }^{\tau} x$, where the critical drop size $\nu_{c}$ is evaluated at threshold. Assuming that the rate of change of $G$ is slow enough (risetime $\gtrsim I s e c$ ) that the size of drops produced remains constant at the up-going value $\nu_{+}$, we use Eq. (3.1) for $n$ to obtain the differential equation

$$
\mathrm{dN} / \mathrm{dt}=\mathrm{C}_{+} \exp \left[\left(\mathrm{G} \tau_{0}-\mathrm{N} \nu_{+}\right) / \mathrm{M}_{+}\right]
$$

This equation can be integrated directly to yield $N$. We break the time integral into two parts: $\int_{0}^{t} r d t \exp \left(G T_{o} / M_{+}\right)$for the time $t_{r} \sim 1$ sec required for $G$ to reach the constant value $G_{U}$, and $\exp \left(G_{U} T_{0} / M_{+}\right) \Delta t$ for subsequent times. Because $G_{U}^{T} / M_{+} \gtrsim \nu_{c}$ above threshold $\left(\nu_{c} \simeq 50\right.$ for $\mathrm{Ge}$ at $\left.2 \mathrm{~K}\right)$, we can neglect the first integral if $\Delta t>t_{r}$. We obtain

$$
N \nu_{+}=M_{+} \ln \left[1+\left(C_{+} \nu_{+} \Delta t / M_{+}\right) \exp \left(G_{U} \tau_{o} / M_{+}\right)\right]
$$

Above threshold the argument of the logarithm in Eq. (3.6) is large (see section 33), and $\mathrm{Eq} .(3.6)$ reduces asymtotically to

$$
N v_{+} \simeq G_{U}^{T}{ }_{0}-M_{+} \ln \left(M_{+} / C_{+} \nu_{+} \Delta t\right)
$$


An interesting feature of Eqs. (3.6) and (3.7) is that the drop concentration $\mathrm{N}$ increases logarithmically with time $\Delta t$. For Ge at $2 \mathrm{~K}, \mathrm{M}_{+} \simeq 10^{11}$ pairs $\mathrm{cm}^{-3}$; thus $\mathrm{N} \nu_{+}$increases by $\sim 2 \times 10^{11}$ pairs $\mathrm{cm}^{-3}$ for each factor of ten increase in $\Delta t$, a change which is large enough to be readily observable. Of course $\mathbb{N}$ cannot increase indefinitely; our assumption that the size $\nu_{+}$remains constant breaks down when $\Delta N v_{+} / t_{0} \sim n / t_{x} ; i . e$. when the additional amount of $E H D$ phase $\Delta N v_{+}$ condensed in the time $\Delta t$ becomes comparable to the $\mathrm{FE}$ density. From Eq. (3.7) and the definition of $M_{+}$we find for $G e$ at $2 \mathrm{~K}$ that the time required is $\Delta t \sim \exp \nu_{c} \sim 10^{22} \mathrm{sec}$, very long indeed.

We can calculate the slow time dependence of the down-going EHD density in a similar manner. Here the rate of change of the drop concentration is given by

$$
\mathrm{dN} / \mathrm{dt}=-\mathrm{J}_{-} \simeq-\mathrm{NC} \mathrm{exp}_{-}\left(-\mathrm{n \tau} \tau_{\mathrm{o}} / \mathrm{M}_{-} \mathrm{\tau}_{\mathrm{x}}\right)
$$

The coefficients $M_{-}$and $C_{-}$are defined in the same way as $M_{+}$and $C_{+}$; we find $M_{-}=\mathfrak{n}_{-} \tau_{0} /\left(\nu_{-}-\nu_{c}\right) \tau_{x}$, where the critical size $\nu_{c}$ is evaluated at the down-going threshold $n_{-}$. The differential equation analogous to $\mathrm{Eq} \cdot(3.5)$ is

$$
\mathrm{dN} / \mathrm{dt}=-\mathrm{NC} \mathrm{Cxp}_{-}\left[-\left(\mathrm{GT} \tau_{0}-\mathrm{N} \nu_{-}\right) / \mathrm{M}_{-}\right]
$$

where we assume $v_{-}$is constant. As before we divide the time integral of Eq. (3.9) into two parts and assume the elapsed time $\Delta t$ is much greater than the fall time (for example $1 \mathrm{sec}$ ) of $\mathrm{G}$ to the constant 


\section{3}

value $G_{D}$. In this way we find

$$
E_{1}\left(N \nu_{-} / M_{-}\right)=E_{1}\left(N_{M} \nu_{-} / M_{-}\right)+C_{-} \Delta t \exp \left(-G_{D} \tau_{0} / M_{-}\right)
$$

where $\mathrm{N}_{M}$ is the initial concentration, and $E_{1}(x) \equiv \int_{x}^{\infty} d y e^{-y} / y$ is the exponential integral. Because $M_{-} \sim 10^{8}$ pairs $\mathrm{cm}^{-3}$ for $\mathrm{Ge}$ at $2 \mathrm{~K}$, the argument of $\mathrm{E}_{1}$ is large for experimentally detectable amounts $\mathrm{NV}$ _ of EHD phase, and we can substitute the asymtotic expression $E_{1}(x) \simeq e^{-x} / x$ into Eq. (3.10). This yields

$$
\left(N_{M}-N\right) \nu_{-} \simeq M_{-} \ln \left\{\left(N / N_{M}\right)+\left(N_{-} \nu_{-} \Delta t / M_{-}\right) \exp \left[-\left(G_{D} \tau_{0}-N_{M} \nu_{-}\right) / M_{-}\right]\right\}
$$

Whenever $\mathrm{N}$ falls appreciably below the initial value $\mathrm{N}_{\mathrm{M}}$, the argument of the logarithm is large, and $\mathrm{Eq} \cdot(3.11)$ becomes approximately

$$
N \nu_{-} \simeq G_{D} \tau_{0}-M_{-} \ln \left(N C_{-} \nu_{-} \Delta t / M_{-}\right)
$$

Note that $\mathrm{N}$ is independent of the initial drop concentration $\mathrm{N}_{M}$ whenever the breakup rate $J_{-}$is appreciable. As in the up-going case, $N V_{-}$from Eq. (3.12) varies logarithmically with time $\Delta t$. However, because $M_{-} \ll M_{+}$, the magnitude of the decrease in $N v_{-}$with time is expected to be much smaller.

These solutions demonstrate that the time required for the FE-EHD system to reach dynamic equilibrium can be extremely long. A lower limit to this time is that value of $\Delta t$ for which the up-going and down-going EHD "signals" N $N$ from Eqs. (3.7) and (3.12) are equal. 
For Ge assuming homogeneous nucleation, the equilibrium time is $\gtrsim 10^{19} \mathrm{sec}$ at $2.0 \mathrm{~K}$, and $\gtrsim 10^{7} \mathrm{sec}$ at $1.5 \mathrm{~K}$. Actual measurements, 3.2 (see chapter 5) of the up-going and downgoing EHD luminescence signals in ultra-pure Ge vary logarithmically with $\Delta t$ as predicted for times in excess of $4 \times 10^{3} \mathrm{sec}$, supporting our theoretical mode1.

\section{Hysteresis and Thresholds}

The up-going (UP) and down-going (DOWN) solutions, Eqs. (3.6) and (3.11) for the EHD signal $\mathrm{N} V$ are plotted versus generation rate for Ge in Fig. 3.1. The upper portion of the down-going curve is obtained from the conservation equation, Eq. (3.1), with $N=N_{M^{*}}$ Here homogeneous nucleation is assumed. In this section and in those to follow we concern ourselves only with the EHD signal at the fixed time of observation $\Delta t=50 \mathrm{sec}$. The vertical scale of Fig. 1(a) is chosen so that this figure can be directly compared to data from sensitive EHD 1uminescence experiments on $\mathrm{Ge},{ }^{3.2,3.3}$ where the noise level is typically $\delta(N V) \sim 3 \times 10^{10}$ pairs $\mathrm{cm}^{-3}$. Hysteresis in the EHD signal $N \cup$ due to the history of excitation is clearly shown in Fig. 3.1(a). Additional drops created by the initially large excitation in the down-going case remain after the generation rate is reduced and cause the large separation between the two curves. Figure 3.1(a) is in good agreement with actual hysteresis data ${ }^{3.2}$ (see chapter 5) taken with the same history of excitation asșumed here.

As shown in Fig. 3.1, both the up-going and down-going signals N $\nu$ display sharp thresholds, $G_{+}$and $G_{-}$, in the generation rate. The 
behavior of $\mathrm{N} v$ near these thresholds is shown in detail by the inserts, Figs. 3.1(c) and 3.1(b), where the horizontal scale has been expanded 10 times and 1000 times respectively. From these graphs it is evident that we can define the thresholds $G_{+}$and $G_{-}$by 1inearly extrapolating to zero the EHD signal above threshold, as shown by the dotted lines In Figs. 3.1(b) and 3.1(c). By comparing the asymtotic expressions, Eqs. (3.7) and (3.12), for $\mathrm{N}$ above threshold with the simple linear forms Eqs. (3.2) and (3.3) we find immediately:

$$
\begin{aligned}
& G_{+}=\left(M_{+} / \tau_{0}\right) \ln \left(M_{+} / C_{+} \nu_{+} \Delta t\right), \\
& G_{-}=\left(M_{-} / \tau_{0}\right) \ln \left(N_{M} C_{-} \nu_{-} \Delta t / M_{-}\right) .
\end{aligned}
$$

In Eq. (3.14) we have substituted the initial value $\mathrm{N}_{\mathrm{M}}$ for the downgoing drop concentration $\mathrm{N}$ in order to make the definition of $\mathrm{G}_{-}$unique. As shown in Fig. 3.1(b), where the dashed line assumes $N=N_{M}$, the variation of $G_{-}$with $\mathrm{N}$ is very small and can be neglected. As noted in chapter 2, the dependence of these thresholds on the time of observation $\Delta t$ is quite small; for $G$ at $2 \mathrm{~K}$, the thresholds $\mathrm{G}_{+}$and $\mathrm{G}_{-}$ from Eqs. (3.13) and (3.14) vary by only $10 \%$ and $0.1 \%$ respectively for a factor of $10^{3}$ change in $\Delta t$.

By substituting the threshold FE densities $n_{ \pm}=G_{ \pm} \tau_{x}$ from Eqs. (3.13) and (3.14) into the original approximate expressions, Eqs. (3.4) and (3.8), for the rates of drop formation $J_{+}$and breakup $J_{-}$, we can put the threshold condition into a more convenient equivalent form: 


$$
J_{ \pm} \nu_{ \pm} \Delta t=M_{ \pm}
$$

Using the exact expressions for $\mathrm{J}_{+}$and $\mathrm{J}_{-}$from chapter 2, Eq. (3.15) can be easily solved numerically for the thresholds $\mathbf{n}_{+}$and $\mathbf{n}_{-}$. Note that Eq. (3.15) is similar to the threshold condition $J_{ \pm} \Delta t=N_{\min }$ used in chapter 2 , except that the fundamental parameters $M_{+}$and $M_{-}$ replace the experimental minimum detectable concentration of drops $\mathrm{N}_{\text {min }}$. Thus thresholds linearly extrapolated from experimental data can be directly compared to those calculated from Eq. (3.15) with no uncertainty introduced by the definition of threshold or by the sensitivity of the apparatus.

At low temperatures $(T \lesssim 1.3 \mathrm{~K}$ for $\mathrm{Ge}$, and $\mathrm{T} \lesssim 4.0 \mathrm{~K}$ for $\mathrm{Si}$ ) the potential barrier to nucleation becomes ineffective, hysteresis in the EHD signa1 NV disappears, and dynamic equilibrium is achieved rapidly. Under these circumstances, a sharp "equilibrium" threshold $G_{e}$ in the generation rate will be observed when the equilibrium concentration of drops $N=h_{s} \Delta \nu$ becomes significant. In order to develop an expression for the threshold condition, we use the approximate form for the EHD signal in dynamic equilibrium,

$$
h_{S} \nu \Delta \nu \simeq C_{e} \exp \left(n \tau_{0} / M_{e} \tau_{x}\right)
$$

where $h_{s}$ and $\Delta \nu$ are the value and width of the size distribution $h_{v}$ at the stable size $v$ (see chapter 2). As before, the coefficients $\mathrm{C}_{\mathrm{e}}$ and $M_{e}$ are determined by expanding the exact expression for $h_{s} \nu \Delta \nu$ about 


\section{$00+300450$}

$-53-$

the threshold to be defined; we find $M_{e}=n_{e} \tau_{o} / \nu_{e} \tau_{x}$, where $n_{e}$ and $\nu_{e}$ are the threshold values of the FE density and the stable drop size. Substituting $\mathrm{n}$ from Eq. (3.16) into the conservation equation, Eq. (3.1), we find

$$
G \tau_{0}=M_{e} \ln \left(h_{s} \nu \Delta \nu / C_{e}\right)+h_{s} \nu \Delta \nu,
$$

which can be solved for $h_{s} \nu \Delta \nu$ as a function of $G$. For values of the EHD signal $N \cup<M_{e}$, the signal $N \nu$ from $E q .(3.17)$ is approximately given by $\mathrm{N} \nu \simeq \mathrm{C}_{\mathrm{e}} \exp \left(\mathrm{G \tau} \mathrm{o}_{\mathrm{o}} / \mathrm{M}_{\mathrm{e}}\right)$. Because $\mathrm{C}_{\mathrm{e}}$ is very small $\left(\mathrm{C}_{\mathrm{e}} \sim 5 \times 10^{-13}\right.$ pairs $\mathrm{cm}^{-3}$ for Ge at $1.2 \mathrm{~K}$ ), the EHD signal bends upward very sharply at threshold and rapid1y approaches a linear dependence on G. We define the equilibrium threshold $G_{e}=n_{e} / T_{x}$ as that value for which the derivative $d\left(h_{s} \nu \Delta \nu\right) / d\left(G_{0}\right)$ is equal to $1 / 2$. From $E q$. (3.17) we thus obtain the threshold condition

$$
h_{s} v_{e} \Delta \nu=M_{e}
$$

where $h_{S}$ is given by either of the exact expressions, Eqs. (2.16) or (2.28), for the size distribution $h_{v}$ in dynamic equilibrium. This threshold condition, Eq. (3.18), is easily solved numerically and does not involve experimental parameters.

\section{Determination of the Surface Tension}

At a given temperature, a measurement of the experimental threshold ratio $G_{+} / G_{-}$can be used to make an accurate determination of the surface 
tension of the e-h 1iquid. The procedure is to fit the ratio of numerical solutions of $\mathrm{Eq}$. (3.15) for $\mathrm{G}_{+}$and $\mathrm{G}_{-}$to the ratio of linearly extrapolated up-going and down-going thresholds from data analogous to Fig. 3.1 taken at a fixed time of observation $\Delta t$. To demonstrate that the threshold ratio accurately determines the surface tension, consider the simple case of homogeneous nucleation for $\mathrm{T} \gtrsim 2.5 \mathrm{~K}$ where the down-going threshold is not appreciably supersaturated, i.e. $\mathrm{n}_{-} / \mathrm{n}_{\mathrm{s}} \simeq 1$. Then we have approximately

$$
\left(\frac{\sigma}{\mathrm{kT}}\right)^{3} \simeq \frac{3}{16 \pi}\left[\mathrm{n}_{\mathrm{o}} \ln \left(\mathrm{n}_{+} / \mathrm{n}_{-}\right)\right]^{2} \ln \left(\mathrm{J}_{\mathrm{o}} \nu_{+} \Delta \mathrm{t} / \mathrm{M}_{+}\right)
$$

where $J_{0}$ is the coefficient of the exponential factor in $J_{+}$from Eq. (2.24). The value of $\sigma$ from Eq. (3.19) is sensitive only to the experimentally well known values of $n_{+} / n_{-}, n_{0}$, and $T$. Because the logarithm is $\sim 50$, uncertainties of several orders of magnitude in the parameters contained in the argument lead to only small errors in $\sigma$. At temperatures $\mathrm{T} \lesssim 2.5 \mathrm{~K}$, the down-going threshold $\mathrm{n}$ is a1so supersaturated, and the value of $\sigma$ depends upon the additional parameters $\tau_{0}$ and the evaporation coefficient $\alpha_{\nu}$ (see chapter 2).

Graphs for Ge of the value of the surface tension $\sigma$ versus the threshold ratio $G_{+} / G_{-}$are shown in Fig. 3.2. These values were calculated from the threshold condition Eq. (3.15) and the exact expressions, Eqs. (2.24) and (2.25), for the rates of drop formation $\mathrm{J}_{+}$and breakup $\mathrm{J}_{-}$; the values of parameters other than $\sigma$ are given in Table 3.1. As shown in Fig. 3.2, the value of $\sigma$ deduced from a given 


\section{6}

threshold measurement $G_{+} / G_{-}$depends surprisingly little upon whether nucleation is assumed to be homogeneous, Fig. 3.2(a), or inhomogeneous, Fig. 3.2(b). These figures also demonstrate the accuracy of this method for determining the surface tension. The curves intersect the vertical axis for $G_{+} / G_{-}=1$ at nonzero values of $\sigma$, because dynamic equilibrium is rapidly established if $\sigma$ is not sufficiently large. Thus, from the single fact that hysteresis is observed in Ge at $1.5 \mathrm{~K}^{3.2}, 3.3,3.19,3.20$ (see also chapter 5), we can conclude that $\sigma \gtrsim 2.1 \times 10^{-4} \mathrm{erg} \mathrm{cm}^{-2}$ at this temperature. Using the procedure described in this section, the author, Staeh1i, and Haller $3.2,3.3$ have made what we believe is the first reliable measurement of the EHD surface tension in Ge, $\sigma=(2.4$ to 2.8$) \times 10^{-4} \mathrm{erg} \mathrm{cm}^{-2}$ at $\mathrm{T}=0$. This value agrees with the more reliable measurement $\sigma=(2.6 \pm 0.3) \times 10^{-4} \mathrm{erg} \mathrm{cm}^{-2}$ at $\mathrm{T}=0$ obtained by fitting the EHD-FE phase diagram as described in chapter 5 .

\section{Modified Phase Diagram}

Previously, $3.8,3.20,3.21$ the low density boundary of the FE-EHD phase diagram was assumed to be given by the saturated FE density $n_{s}$ (see chapter 2). From the foregoing discussion it is clear that metastability and the EHD lifetime have a major effect on the regions of stability of the FE-EHD system. We can construct a modified "phase diagram" which correct1y accounts for these effects by plotting the thresholds $n_{ \pm}=G_{ \pm}{ }^{\tau}$ from section 33 versus reciprocal temperature. The results for Ge are shown in Fig. 3.3 for both the homogeneous and inhomogeneous cases. The values of $n_{+}$and $n_{-}$were obtained by 
numerically solving the threshold condition Eq. (3.15). Both the upgoing and down-going thresholds, Figs. 3.3(a) and 3.3(b) respectively, occur at FE densities well above the saturated value $n_{s}, F i g .3 .3(c)$. Drops are always present when the FE density $\mathrm{n}$ exceeds $\mathrm{n}_{+}$, and are never present when $\mathrm{n}<\mathrm{n}_{-}$. In the region between the up-going and down-going thresholds, the presence of drops depends upon the history of excitation. As explained in chapter 2 and section 32 , the variation of the thresholds $n_{+}$and $n_{-}$is relatively small for times of observation in the range $10 \mu \mathrm{sec}<\Delta t<100 \mathrm{sec}$. Significant changes in $\mathrm{n}_{+}$and $\mathrm{n}_{-}$ with $\Delta t$ occur only near the point where the two curves join. Thus the up-going curve of Fig. 3.3 applies to experiments performed with pulsed excitation (pulse risetime $\gtrsim 10 \mu \mathrm{sec}$ ), as we11 as those with continuous excitation.

At temperatures below $2 \mathrm{~K}$ the separation of the up-going and downgoing thresholds, Figs. 3.3(a) and 3.3(b), decreases, disappearing at $1.3 \mathrm{~K}$ for the homogeneous case and at $1.4 \mathrm{~K}$ for the inhomogeneous case. For lower temperatures the FE-EHD system is in dynamic equilibrium, and the threshold for drop formation in Fig. 3.3 is given by the equilibrium threshold $\mathrm{n}_{\mathrm{e}}$ from solutions of $\mathrm{Eq} \cdot(3.18)$. In addition, the curves increasingly deviate from the saturated FE density, Fig. 3.3(c), as the temperature is lowered; near $\mathrm{T} \sim 1 \mathrm{~K}$ the equilibrium threshold becomes nearly temperature-independent. Both these phenomena are caused by the presence of the lifetime term in $\psi_{\nu}$, the effective free energy difference between a drop of size $v$ and $v$ free excitons, as explained in chapter 2. Preliminary measurements $3.2,3.3,3.19$ for Ge of the up-going and down-going thresholds in 
excitation level versus temperature display all of the qualitative features of Fig. 3.3, supporting this theoretical model. In chapter 5 we find excellent quantitative agreement between theory and threshold data.

Contrary to what one might expect, the phase diagrams obtained by assuming homogeneous and inhomogeneous nucleation are quite similar. The primary effect of the binding energy $\mathrm{B}_{v}$ of drops to impurities (see chapter 2) is to reduce the supersaturation ratio of the up-going threshold, Fig. 3.3(a), at low temperatures and thereby increase slight1y the temperature at which hysteresis disappears. The value of the barrier lowering due to impurities $\Delta \psi=B_{v}-k T I n Z$ (see chapter 2) was assumed to be temperature independent in calculating Fig. 3.3. It is possible that impurities saturate with small exciton complexes before macroscopic drops form. If this is so, then the temperature dependence of the term $\mathrm{kTlnz}$ in $\Delta \psi$ could be important at low $\mathrm{T}$. Finally note that the similarity of the two phase diagrams of Fig. 3.3 makes difficult the experimental determination of the type of nucleation from the temperature dependence of threshold data.

As discussed in chapter 1 , previous threshold measurements $3.8,3.20,3.21$ of the EHD condensation energy $\phi$ were made by fitting data apparently taken on the up-going curve, Fig. 3.3(a), to the saturated FE density, Fig. 3.3(c). The values obtained, typically $\phi \simeq 17 \mathrm{~K}$, disagree with the spectroscopic measurements, $3.4,3.8,3.9$ typically $\phi \simeq 23.5 \mathrm{~K}$ at $\mathrm{T}=2 \mathrm{~K}$, which were used in calculating Fig. 3.3. The apparent value of $\phi$ deduced in this way from the slope of the up-going threshold, Fig. 3.3(a), 
between $2 K$ and $4 K$ is $\phi \simeq 15 K$. As pointed out earlier, $3.2,3.3$ the failure to understand and use hysteresis effects seems to explain the apparent discrepancy between spectroscopic the older threshold measurements of the condensation energy.

When the temperature-independent evaporation rate ${ }^{3.22} c \nu^{2 / 3}$ is included in the calculation as discussed in chapter 2, the thresholds are modified. Figure 3.4 illustrates the effect of this term on the phase diagram in Ge for various values of the coefficient c. For $\mathrm{c}<5 \times 10^{4} \mathrm{sec}^{-1}$ the calculated thresholds between $1.25 \mathrm{~K}$ and $2 \mathrm{~K}$ are practically independent of $c$. However, as c increases from $5 \times 10^{4} \mathrm{sec}^{-1}$ to $5 \times 10^{6} \mathrm{sec}^{-1}$, the low-temperature plateau of the phase diagram moves to higher FE density, the threshold separation decreases, and the temperature at which hysteresis disappears increases. As discussed in chapter 5, actual low temperature threshold data show evidence for these effects, and a fit of theory to these data yields $c \sim 10^{5} \sec ^{-1}$. Phase diagrams for the FE-EHD system in $\mathrm{Si}$, calculated in the same way as for Ge, are shown in Fig. 3.5. These curves exhibit all of the qualitative features of the phase diagrams for Ge, Fig. 3.3. These features occur at higher temperatures simply because the condensation energy for $\mathrm{Si}^{3.8} \phi \approx 79 \mathrm{~K}$ is approximately 4 times greater than for Ge. Because the values of the parameters used to calculate Fig. 3.5 were not as well established as those for Ge, these phase diagrams are expected to be less accurate than Fig. 3.3. However, these values have been somewhat justified by recent measurements and calculations as discussed in the introduction to this chapter. The 1argest error 


\section{0 \\ $-59-$}

expected in Fig. 3.5 is that due to the value used for $\phi$, which was

$\sim 16 \mathrm{~K}$ smaller than the recent measurement ${ }^{3.10} \phi=95 \mathrm{~K}$ at $\mathrm{T}=0$.

Nevertheless, these phase diagrams display two qualitative features of major interest. First, metastability of the FE-EHD system in Si disappears for temperatures below $\mathrm{T} \simeq 5 \mathrm{~K}$; thus no hysteresis effects are expected at 1iquid He temperatures. Second, and more importantly, the threshold for drop formation in $\mathrm{Si}$ below $5 \mathrm{~K}$ occurs at a measurably high FE density, $\mathrm{n}_{\mathrm{e}} \sim 2 \times 10^{13} \mathrm{~cm}^{-3}$, which is approximately temperature independent. If drop formation occurred at the saturated FE density, the threshold at $2 \mathrm{~K}$ would be unphysically small, $\mathrm{n}_{\mathrm{s}} \simeq 0.6 \mathrm{~cm}^{-3}$. To our knowledge no measurements have been reported of the phase diagram in Si which could be directly compared to Fig. 3.5. However, EHD thresholds have apparently been observed ${ }^{3.8}$ at $2 \mathrm{~K}$ for moderate levels of excitation. Also threshold measurements ${ }^{3.23}$ at temperatures above $15 \mathrm{~K}$ agree reasonably well with the threshold $\mathrm{FE}$ densities shown in Fig. 3.5,

\section{Drop Radius}

A plot of the EHD stable radii at the up-going (UP) and down-going (DOWN) thresholds, $R_{+}$and $R_{-}$respectively, is shown for Ge in Fig. 3.6. Below $T \sim 1.3 \mathrm{~K}$ hysteresis vanishes and the drop radius shown is $R_{e}$, the value at the equilibrium threshold. The values of $R_{+}, R_{-}$, and $R_{e}$ were numerically computed from the expression Eq. (2.17) for the free energy difference $\psi_{v}$ at the FE density thresholds shown in Fig. 3.3. The distribution of drop sizes is very sharply peaked about the stable 
radius; at $2 \mathrm{~K}$ the width is only $\delta \mathrm{R}_{+} \sim 1 \times 10^{-3} \mu \mathrm{m}$. The radii predicted for homogeneous, Fig. 3.6(a), and inhomogeneous nucleation, Fig. 3.6(b), are quite similar, a consequence of the similarity of the phase diagrams, Fig. 3.3, for the two cases. As explained in chapter 2, the stable drop radius $R$ is determined by the density of the surrounding FE gas. Above $T \sim 1.5 \mathrm{~K}$ the following approximate expression ${ }^{3.8}$ is valid:

$$
R \simeq r_{0} \beta \tau_{0}\left(n-n_{s}\right)
$$

where $\beta \equiv \pi \mathrm{r}_{\mathrm{o}}^{2}(8 \mathrm{kT} / \pi \mathrm{m} *)^{1 / 2}$ is the coefficient of the rate at which $\mathrm{FE}$ are absorbed by the drop. The strong temperature dependence of the radii shown in Fig. 3.6 is thus a direct consequence of the variation of the FE density thresholds with temperature, Fig. 3.3.

In an actual experiment one would expect to observe the radii shown in Fig. 3.6 only if the excitation level were very slowly and smoothly varied as described in section 31. If the excitation is increased more rapidly in the up-going case, a larger number of drops smaller than $\mathrm{R}_{+}$form, and the drop size depends upon the risetime and magnitude as we11 as the history of the excitation. ${ }^{3.16}$ Thus, under more general circumstances, the up-going and down-going radii of Fig. 3.6 are upper and lower limits to the steady state drop size. Drop radii observed in experiments using intense focussed excitation 3.17 and short laser pulses ${ }^{3.6}$ are most likely determined by mechanisms other than nucleation.

At temperatures above $T \sim 2.5 \mathrm{~K}$, the calculated up-going radius $R_{+}$ shown in Fig. 3.6 becomes quite large. Because the decay of e-h pairs 


\section{9}

within a drop must be supplied by diffusion of excitons to its surface, the radius of a large drop is limited by the 1ocal depletion of FE. We can find the diffusion-limited up-going radius $\mathrm{R}_{\mathrm{D}}$ by solving the steady-state diffusion equation. The appropriate boundary conditions on the FE density $n(r)$ are $n=n_{+}$for $r \rightarrow \infty$, and $\left(4 \pi R^{2} D\right) d n / d r=\nu / \tau_{0}$ for $\mathrm{r}=\mathrm{R}$, where $\mathrm{D}$ is the diffusion constant. The latter condition states that the current of $\mathrm{FE}$ to the drop surface equals the decay rate $\nu / \tau_{0}$. Using $\mathrm{Eq} \cdot(3.20)$ for the drop radius we find

$$
R_{D} \simeq \frac{1}{2} \frac{3 D}{r_{0} \beta n_{o}}\left\{-1+\left[1+\frac{4 r_{o} \beta \tau_{o}\left(n_{+}-n_{s}\right)}{\left(3 D / r_{o} \beta n_{o}\right)}\right]^{1 / 2}\right\} .
$$

This expression reduces to $\mathrm{Eq} \cdot(3.20)$ at low temperatures. Below threshold at $3 \mathrm{~K}$ the $\mathrm{FE}$ diffusion constant in Ge is ${ }^{3.8} \mathrm{D} \sim 1500 \mathrm{~cm}^{2} \mathrm{sec}^{-1}$ due to phonon scattering. At higher temperatures where the FE density above threshold is large, D must be determined by self diffusion. From the simple kinetic theory of gases the self-diffusion constant is

$$
\mathrm{D}=\left(4 \mathrm{kT} / \pi \mathrm{m}^{*}\right)^{1 / 2} / 3 \mathrm{cn}_{+},
$$

where we use $c \sim 5 \times 10^{-11} \mathrm{~cm}^{2}$ for the $\mathrm{FE}-\mathrm{FE}$ collision cross section. Because the drop radius varies from 0 to $R_{+}$as $n$ increases by only $\sim 50 \%$ from $\mathrm{n}_{\mathrm{S}}$ to $\mathrm{n}_{+}$, we have substituted the threshold value $\mathrm{n}_{+}$into Eq. (3.22). The resulting error in the computed radius is $\sim 25 \%$. The diffusion limited radius calculated from Eqs. (3.21) and (3.22) is 
shown as the dashed curve in Fig. 3.6. While ineffective below $\mathrm{T} \lesssim 2.5 \mathrm{~K}$, FE diffusion limits the drop radius at higher temperatures to the nearly constant value $R_{D} \simeq 15 \mu \mathrm{m}$. The curve $R_{D}$ in Fig. 3.6 is in reasonable agreement with the light-scattering measurements of Bagaev et al. 3.24 Using volume excitation chopped at $1 \mathrm{kHz}$, they found that the drop radius increased from $R=4 \mu \mathrm{m}$ at $2.6 \mathrm{~K}$ to $\mathrm{R}=10 \mu \mathrm{m}$ at 3.5K. Because the risetime of their pulsed excitation is shorter than the minimum value for which Fig. 3.6 is directly applicable, it is reasonable that their measurements of $R$ lie somewhat below $R_{D}$ (see above) .

As shown in $F i g .3 .6$, at low temperatures the drop radii $R_{+}$, $R_{-}$, and $\mathrm{R}_{\mathrm{e}}$ become quite small; $\mathrm{R}_{\mathrm{e}}$ approaches $0.02 \mu \mathrm{m}$ at $\mathrm{T}=1 \mathrm{~K}$, which corresponds to the size $\nu \sim 10 \mathrm{e}-\mathrm{h}$ pairs. As the drop radius becomes small, the fraction $\sim 3 r_{0} / R$ of $e-h$ pairs within the surface thickness $\sim_{r_{0}}$ of the drop becomes signiftcant, causing a change in spectroscopic lineshape and a shift to higher energy of the EHD luminescence. This effect has been observed experimentally by Etienne et al. ${ }^{3.25}$ who accounted for the existence of hysteresis. They deduced down-going drop radii increasing from $R_{-} \simeq 0.025 \mu \mathrm{m}$ at $1.43 \mathrm{~K}$ to $R_{-} \simeq 0.1 \mu \mathrm{m}$ at 2.0K. These values of $R_{\text {_ }}$ are a factor of 2 to 3 smaller than those of Fig. 3.6, but show the correct temperature dependence. Although the analysis in Ref. 3.25 seems questionable, their data indicate that drop radii do indeed become quite small at low temperatures in agreement with our theoretical prediction. 
CHAPTER 4. EXPERIMENTAL APPARATUS

All of the experimental results presented in this thesis were obtained by studying the near-infrared luminescence of electron-hole drops and free excitons in optically excited Ge. This method of excitation and detection of $\mathrm{EHD}$ and $\mathrm{FE}$ offers the advantages of flexibility, very high sensitivity, and unambiguous identification of the species of interest. A simplified schematic diagram of the apparatus is shown in Fig. 4.1. This diagram can be divided into three basic functional blocks: the source of excitation, consisting of the tungsten lamp, the Ge monitor detector, and associated optics and electronics; the samples, cooled to 1fquid He temperatures in an optical cryostat; and the detection apparatus, consisting of the spectrometer, the Ge luminescence detector, and the signa1 processing electronics. These three functional blocks will be described in detail in sections 41,42 , and 43, respectively.

The fact that this apparatus was used to study hysteretic phenomena in the EHD-FE system placed a number of highly unusua1 constraints on the design of its components. As we show later, the state of the FE-EHD system in Ge is sensitive to the entire history of excitation of the sample for times as 1 ong as $\sim 10^{6} \mathrm{sec}$ preceeding a measurement, even though the response times of this system are of the order of $10^{-6} \mathrm{sec}$. Thus the excitation must be cycled in an accurately reproducible manner as illustrated in Fig. 4.1 and described in chapter 3, and the source of excitation must be nearly noise-free. 
In order to study the metastable time development of the EHD luminescence intensity for periods of time of the order $10^{3} \mathrm{sec}$, the excitation level and temperature must be carefully stabilized. Similarly the Ge samples must be free of any external mechanisms such as electrical contacts, which are known to destroy drops and thus erase the memory of the system. Considerable effort was also expended developing a very high sensitivity luminescence detection apparatus in order to study the $\mathrm{FE}-\mathrm{EHD}$ system near threshold at temperatures below $2 \mathrm{~K}$, where the luminescence signals are exceedingly small.

\section{Optical Excitation}

When a crystal of ultrapure Ge at liquid He temperatures is illuminated by visible or near-infrared radiation, free carriers are excited; in this wavelength range one electron-hole pair is created for each photon absorbed. If the photon energy is greater than the direct energy gap. $\mathrm{E}_{\text {gap }}^{\mathrm{d}} \approx 890 \mathrm{meV}$, this process can occur without phonon emission, and the absorption length of the radiation is correspondingly short, $\quad \ell \lesssim 1 \mu \mathrm{m}$; we refer to this case as surface excitation. The electrons thus created rapidly relax to the indirect band edge and form free excitons within several $\mu \mathrm{m}$ of the illuminated surface. These excitons subsequently move $\sim 1 \mathrm{~mm}$ into the crystal and recombine. Assuming this motion is diffusive, the spatial form $n(\vec{x})$ of the steady-state $\mathrm{FE}$ concentration below the threshold for drop formation is determined by the diffusion constant ${ }^{4.1} \mathrm{D} \approx 1500 \mathrm{~cm}^{2} \mathrm{sec}^{-1}$ at $3 \mathrm{~K}$ and the FE 1ifetime $\tau_{\mathrm{x}} \sim 5 \mu \mathrm{sec}$, and $\mathrm{n}(\overrightarrow{\mathrm{x}})$ is everywhere proportional 
to the e-h pair generation rate $G$ at the surface. Thus we can establish a quantitative relationship between the exciton concentration $\mathrm{n}(\overrightarrow{\mathrm{x}})$ and the excitation 1evel. A more direct method of excitation occurs if the incident photon energy is less than the direct gap $E_{\text {gap }}^{d}$, but substantially greater than the indirect gap $\mathrm{E}_{\text {gap }}^{i} \approx 744 \mathrm{meV}$. Then direct absorption is impossible, but electrons can be excited into the energy minimum at the zone edge. Because this process involves phonon emission, the absorption length is much 1onger, $\ell \gtrsim 0.1 \mathrm{~mm}$; we refer to this case as volume excitation. For the lowest excitation levels used in these experiments, the formation time of $\mathrm{FE}$ in this case is still very short $(\lesssim 1 \mu \mathrm{sec})$. However the FE diffusion length $\mathrm{L}=\sqrt{\mathrm{D} \tau_{\mathrm{x}}} \sim 1 \mathrm{~mm}$ can be made comparable to or smaller than $\&$ by using the longest wavelength light which gives a reasonable level of excitation. If $\ell \gtrsim L$ the spatial form of $n(\vec{x})$ is determined primarily by the volume generation rate $\mathrm{G}(\overrightarrow{\mathrm{x}})$, and effects of $\mathrm{FE}$ transport are minimized.

For either type of excitation, the fundamental concept relating experiment to nucleation theory is that the incident radiation creates in the Ge crystal a FE gas of concentration $n(\vec{x})$ which, below threshold, is everywhere proportional to the excitation level.

Volume excitation is clearly preferable for studies of EHD nucleation phenomena, and was used whenever possible in taking the data presented in this thesis. This method of excitation minimizes the influence of possible undesirable surface effects such as the trapping of generated e-h pairs and the destruction of $\mathrm{FE}$. Also, data taken in 
this way can be directly compared with the nucleation theory of chapters 2 and 3, which assumes a spatially uniform $\mathrm{FE}$ gas, and the difficult problem of analyzing the coupled transport of $\mathrm{FE}$ and drops from the excited surface can be partially avoided. Finally, it was deemed necessary to use volume excitation in order to prove conclusively that the observed luminescence hysteresis was an intrinsic property of the FE-EHD system itself, not an artifact related to the sample surfaces.

As mentioned in the introduction to this chapter, the study of nucleation phenomena in the EHD-FE system imposes important restrictions on the manner in which the samples can be excited. The theoretical reasons for these restrictions are the excitation memory inherent in hysteretic effects and the metastable time variation of the EHD signal for a given fixed excitation level. The most obvious way to generate hysteresis curves for the EHD luminescence intensity $I_{d}$ vs. excitation level $P$ is to continuously record $I_{d}$ as $P$ is slowly swept from zero to a maximum value $\mathrm{P}_{\text {max }}$, then back to zero; this method was used in previous work. $4.2,4.3$ However, because $I_{\mathrm{d}}$ varies with time, the results obtained depend upon the sweep rate in a complicated way, and cannot easily be compared with theory. Therefore, the data presented in this thesis were taken point-by-point as described in chapter 3 and illustrated in Fig. 4.1. Each "upgoing" (UP) point was taken by slowly and monotonically increasing the excitation from zero to a constant value $P$, recording the EHD signal $I_{d}$, then returning the excitation to zero to erase the memory of the system. This cycle is then repeated for different values 
of $\mathrm{P}$. Each downgoing (DOWN) point was taken by initially increasing the excitation from zero to a maximum value $\mathrm{P}_{\max }$, then monotonically decreasing the excitation to a constant value $P$ at which $I_{d}$ is recorded, then returning to $\mathrm{P}=0$; the cycle is repeated with the same value for $\mathrm{P}_{\text {max }}$, but different $\mathrm{P}$. Because the EHD-FE system rapid1y responds to and "remembers" changes in $\mathrm{P}\left(\tau_{\mathrm{x}} \sim 5 \mu \mathrm{sec}\right)$, the variation of $\mathrm{P}$ with time must be accurately monotonic, and the source of excitation must be free of noise spikes. Also, the final EHD signal recorded has been shown theoretically ${ }^{4.4}$ (see chapter 3) and experimenta11y ${ }^{4.5}$ to be sensitive to the rate of change of the excitation, if this rate is too rapid. In chapter 3 we found that $I_{d}$ is expected to depend upon the leading edge of an exciting pulse at $2 \mathrm{~K}$ for risetimes shorter than only $0.04 \mathrm{sec}$. Therefore changes in the up- and downgoing excitation cycles must be made very slowly and reproducib1y, as shown in Fig. 4.1, in order to obtain reproducible results. Finally the excitation $P$ must be held accurately constant for times $\sim 10^{3} \mathrm{sec}$ in order to measure very sma11 metastable changes in the EHD Iuminescence intensity with time.

These excitation requirements were met with a system, shown in Fig. 4.1, based upon a tungsten-halogen lamp. This light source has the advantages of intrinsic stability and low noise, and can be easily electronically regulated. The lamp radiates as a black body of temperature $\mathrm{T}$ rough1y proportional to the input power; at the maximum operating temperature $\mathrm{T} \approx 3200 \mathrm{~K}$, the photon $\mathrm{flux}$ spectrum is peaked at the wavelength $\lambda \approx 2.8 \mu \mathrm{m}$, and falls exponentially for shorter $\lambda$. 
Because the useful wavelengths are shorter than $1.6 \mu \mathrm{m}$, the usable lamp output is a highly non-linear function of the input voltage, and feedback regulation is desirable. The actual lamp used was a $12 \mathrm{~V}$, 100W, tungsten-iodide projection lamp with a fused Quartz envelope, manufactured by Eumig, mode1 P8 Novo. Any tungsten-halogen lamp with a small, closely wound filament and similar characteristics would be suitable. This lamp was mounted in a fan-cooled chimney; the light was collected and refocused by two aspheric plano-convex Quartz lenses of focal length $3^{\prime \prime}$ and diameter $3^{\prime \prime}$, mounted with their convex sides facing. The light beam was approximately collimated by a doubleconcave glass lens of focal length $38 \mathrm{~mm}$ and diameter $33 \mathrm{~mm}$, positioned near the focal point of the second Quartz lens; the final beam diameter thus obtianed was $\approx 2 \mathrm{~cm}$.

Volume excitation was achieved by passing the collimated light beam from the lamp through a four-cavity interference filter of bandpass (FWHM) $40 \mathrm{~nm}$ centered about the wavelength $\lambda=1.52 \mu \mathrm{m}$ as shown in Fig. 4.1. This value of $\lambda$ was selected such that the photon energy $\approx 810 \mathrm{meV}$ was slight1y smaller than the direct energy gap in Ge at $4.2 \mathrm{~K}$; thus absorption is an indirect process as described earlier. The bandwidth was chosen to insure that a negligible fraction of the radiation was surface absorbed, and also to limit the amount of light transmitted through the Ge samples, typically $3 \mathrm{~mm}$ thick. In addition, a $1 \mathrm{~cm}$ thick disc of antireflection coated high-purity $\mathrm{Si}$ was placed in the light beam to remove remnants of surface-absorbed radiation of wavelength $\lesssim 1.0 \mu \mathrm{m}$. A measurement of the resulting 


\section{$00+3404013$}

excitation spectrum is shown in Fig. 4.2. The measured value of the absorption length of this radiation in ultra-pure Ge at $4.2 \mathrm{~K}$ increased from $0.85 \mathrm{~mm}$ to $1.30 \mathrm{~mm}$ over the bandpass (FWHM) of the interference filter. Thus the generation rate $G\left(\mathrm{~cm}^{-3} \mathrm{sec}^{-1}\right)$ of e-h pairs within uniformly illuminated Ge samples is well approximated by the expression

$$
G(x)=G_{0} \exp (-x / \ell)
$$

where $\ell \approx 1.1 \mathrm{~mm}, \mathrm{x}$ is the distance from the illuminated surface, and $G_{0}=P / h v l$, with $P\left(W \mathrm{~cm}^{-2}\right)$ the absorbed excitation power density and $h \nu$ the mean photon energy. The maximum total power attainable with this apparatus was $\approx 5 \mathrm{~mW}$, measured after the interference filter.

The steady-state $\mathrm{FE}$ concentration $\mathrm{n}(\mathrm{x})$ produced below EHD threshold by the generation rate $G(x)$ from $E q .(4.1)$ can be calculated by solving the one-dimensional FE diffusion equation. Assuming that the surface recombination rate of $\mathrm{FE}$ is negligible (see section 42), the appropriate boundary condition at $\mathrm{x}=0$ is $\mathrm{dn} / \mathrm{dx}=0$; $i . e$. the net current of $\mathrm{FE}$ is zero at the surface. Then

$$
n(x)=G_{0}{ }^{\top} x \frac{\ell / L}{1-(\ell / L)^{2}}[\exp (-x / L)-(\ell / L) \exp (-x / \ell)]
$$

where $L=\left(D_{x}\right)^{1 / 2}$ is the FE diffusion length. From this expression we find that $n(x)$ is practically constant near the illuminated surface; with $\mathrm{L}=0.7 \mathrm{~mm}$, the $\mathrm{FE}$ concentration at $\mathrm{x}=0.5 \mathrm{~mm}$ is on $1 \mathrm{y} 10 \%$ smaller than that at $x=0$. Thus this method of volume excitation creates a nearly uniform concentration of $\mathrm{FE}$ in a relatively large volume of the 
excited crystal.

When a total power larger than that possible with $1.52 \mu \mathrm{m}$ radiation was required, surface excitation was employed. This was achieved by replacing the $\mathrm{Si}$ and interference filters shown in Fig. 4.1 by a $7 \mathrm{~cm}$ thick filter of distilled water; otherwise the apparatus was unchanged, The water filter altenuates radiation of wavelength greater than $1.4 \mu \mathrm{m}$ by a factor in excess of $10^{15}$; thus the power transmitted through the Ge sample to the detector is negligible and cannot obliterate the desired luminescence signals. The result is a smooth broad excitation spectrum which varies somewhat with excitation power; the photon flux increases with $\lambda$ through the visible range, peaks at $\lambda \approx 0.85 \mu \mathrm{m}$, and is cut off for $\lambda \gtrsim 1.0 \mu \mathrm{m}$. At the peak wavelength, the absorption length in Ge at $4.2 \mathrm{~K}$ is $\ell \approx 0.5 \mu \mathrm{m}$; the value of $\ell$ varies from $\ell \approx 0.1 \mu \mathrm{m}$ at $\lambda=500 \mathrm{~nm}$ to $\ell \approx 1.0 \mu \mathrm{m}$ at $\lambda=1.0 \mu \mathrm{m}$. No evidence of e-h trapping due to surface states was observed with this excitation spectrum, i.e. the FE luminescence intensity was accurately proportional to $\mathrm{P}$ below threshold at all temperatures below 4.2K. Neglecting surface recombination, the steady state $\mathrm{FE}$ density profile $n(x)$ is given by $\mathrm{Eq} \cdot(4.2)$ in the limit $\ell \ll \mathrm{L}$ :

$$
n(x)=\left(G_{0} \ell \tau_{x} / L\right) \exp (-x / L)
$$

where $G_{0} l \approx P / h \nu$, with $\mathrm{P}\left(\mathrm{W} \mathrm{cm}^{-2}\right)$ the absorbed power density and $\mathrm{h} \nu$ the photon energy corresponding to $\lambda=0.85 \mu \mathrm{m}$. Note that $\mathrm{n}(\mathrm{x})$ from $\mathrm{Eq} \cdot(4.3)$ decreases linearly with $x$ near the sample surface; thus the region of uniform $\mathrm{FE}$ concentration is much smaller than for volume excitation. 
The maximum total power attainable in this case was $\approx 200 \mathrm{~mW}$, measured after the water filter.

For both volume and surface excitation, the light beam was focussed onto the Ge sample mounted in an optical cryostat by an aspheric planoconvex Pyrex condenser of focal length $65 \mathrm{~mm}$ and diameter $72 \mathrm{~mm}$, as shown in Fig. 4.1. The size of the excitation beam on the Ge crystal was adjusted by varying the position of the Pyrex lens to be approximately $1 \mathrm{~cm} \times 1 \mathrm{~cm}$, several times the size of the sample; thus the entire front surface of the sample was uniformly illuminated in all cases. This excitation geometry is highly favorable for threshold measurements on the EHD-FE system. As the excitation is increased an entire plane inside the crystal passes through the threshold FE concentration at once, vs. a simple point for spot excitation; therefore the EHD luminescence, collected from the entire crystal, displays a sharper threshold. This geometry greatly increases the sensitivity of the apparatus, by increasing the sample volume occupied by $\mathrm{FE}$ and EHD.

An essential feature of this apparatus is the accurate measurement of the e-h pair generation rate within the Ge sample, and thus the $\mathrm{FE}$ concentration. This was achieved by using a Ge monitor detector as shown in Fig. 4.1. Approximately 10\% of the exciting radiation was removed from the beam by a thin plate-glass beamsplitter and focussed onto the detector by an aspheric Pyrex condenser of focal length $65 \mathrm{~mm}$ and diameter $72 \mathrm{~mm}$. The detector itself was a large $\left(10 \times 10 \times 3 \mathrm{~mm}^{3}\right)$ p-i-n photodiode fabricated from ultrapure Ge by E. E. Haller at Lawrence Berkeley Laboratory, mounted in vacuum, cooled to approximately $80 \mathrm{~K}$ in a small liquid $\mathrm{N}_{2}$ cold-finger dewar, and reverse biased 
with $15 \mathrm{~V}$. The mounting geometry was such that the radiation entered a $3 \times 10 \mathrm{~mm}^{2}$ face of the detector and passed between the junctions, which were applied to the $10 \times 10 \mathrm{~mm}^{2}$ sides; thus the monitor is sensitive to volume absorbed light. Under these conditions the diode current I is proportional to the total rate of generation of carriers within the intrinsic region. Because the monitor was made of the same material as the Ge samples, the monitor current $I_{M}$ is insured to be accurately proportional to the total carrier generation rate within the excited samples as desired. This characteristic is important for surface excitation, where the spectral character of the light varies with excitation level.

In order to relate the monitor current to the absolute FE concentration in the samples, the excitation apparatus was calibrated with a Scientech 1 inch optical calorimeter. This instrument measures optical power and has a flat wavelength response from the ultra-violet to the far infrared. The calorimeter was placed after the empty dewar in the position occupied by the chopper in Fig. 4.1, and recorded the total excitation power transmitted through a hole in the sample holder from which the Ge sample had been removed, otherwise the optics were identical to those used for taking data. The resulting reading was corrected for the transmission of the three exit windows of the dewar and the exit lens, assuming a 10\% loss in each element. The absorbed power density $\mathrm{P}\left(\mathrm{mW} \mathrm{cm}^{-2}\right)$ was then calculated from the area of the hole, $0.25 \mathrm{~cm}^{2}$, and the ideal transmission of one Ge surface, $64 \%$. For volume excitation, the result was 


\section{3}

$-73-$

$$
P\left(\mathrm{~mW} \mathrm{~cm}{ }^{-2}\right)=0.079 \mathrm{I}_{\mathrm{M}}(\mu \mathrm{A})
$$

For surface excitation with $I_{M} \sim 50 \mu \mathrm{A}$,

$$
\mathrm{P}\left(\mathrm{mW} \mathrm{cm} \mathrm{cm}^{-2}\right)=0.144 \mathrm{I}_{\mathrm{M}}(\mu \mathrm{A})
$$

The difference between these results is entirely accounted for by the difference between the average photon energies, $h \nu=0.816 \mathrm{eV}$ and $\mathrm{h} \nu=1.46 \mathrm{eV}$ respectively. Finally, the peak $\mathrm{FE}$ concentration within the samples was calculated from Eq. (4.4) or (4.5) by using Eq. (4.2) or (4.3) respectively, with the result for volume excitation:

$$
\mathrm{n}(0)=\left(\mathrm{Pt}_{\mathrm{x}} / \mathrm{h} \nu\right) /(l+\mathrm{L})
$$

and for surface excitation:

$$
\mathrm{n}(0)=P \tau_{\mathrm{x}} / \mathrm{h} \nu \mathrm{L}
$$

The value of $\mathrm{T}_{\mathrm{x}}$ was measured in each sample used, and the value 4.1 $D=1500 \mathrm{~cm}^{2} \mathrm{sec}^{-1}$ was used for the $\mathrm{FE}$ diffusion constant which was assumed to be temperature-independent; together these numbers determine $L=\left(D \tau_{x}\right)^{1 / 2}$. The overall absolute accuracy of this calfbration procedure for volume excitation is probably limited to roughly a factor of two by uncertainties in the diffusion model for the transport of $\mathrm{FE}$, the value of $\mathrm{D}$, and the unknown mechanism which determines $\tau_{\mathrm{x}}{ }^{*}$ For surface excitation only, the possible surface recombination of $\mathrm{FE}$ introduces an additional source of exror which could be quite large. 
Control of the excitation level during an experiment was automated by a specially-constructed electronic apparatus which accomplished two major goals in the design of this system: 1) feedback stabilization of the excitation, and 2) accurately reproducible cycling of the excitation level. As shown in Fig. 4.1, feedback stabilization was achieved by comparing the output of the monitor detector to the output of a reference function generator, and by using the amplified difference to control the voltage of a programmable DC power supply (Hewlett-Packard/Harrison model 6260A) which drove the tungsten lamp.

A block diagram of the electronic apparatus constructed showing the connections to other equipment is given in Fig. 4.3. Circuit diagrams for the four basic functional blocks shown in Fig. 4.3, the reference function generator, the current amplifier for the monitor detector, the variable-gain differential amplifier, and the timing logic, are given in Figs. 4.4a to $4.4 \mathrm{c}$ and Fig. 4.5 respectively.

Stabilization of the lamp output to the level determined by the function generator was accomplished by the current and the differential amplifiers. The current amplifier, Fig. 4.4b, is in a standard configuration, with selectable full scale currents of $0.1,1,10$, and 100 a corresponding to $1 \mathrm{~V}$ output. A separate current range switch is enabled while logic signal LI is high, so that the initial excitation in the downgoing case can be set at a level much larger than that at which the signal is recorded. The differential amplifier, Fig. 4.4c, consists of two operational amplifiers, one wired for a fixed voltage gain of 10 , and one with a gain adjustable from 1 to 100 , so that the total gain 
can be varied from 10 to 1000 . The output $\mathrm{V}_{\mathrm{PS}}$ is clipped at $12.5 \mathrm{~V}$ and $-0.6 \mathrm{~V}$ by the Zener diode to protect the lamp and the power supply. In order to achieve the best regulation the gain was set at the largest value at which the feedback system was stable, determined by monitoring the voltage $V_{I}$ on an oscilliscope during an excitation cycle. The values of feedback gain thus determined were $\approx 200$ for volume excitation and $\approx 125$ for surface excitation. With these gain settings the performance of the system was excellent: the unregulated drift in the lamp output, $\approx 5 \%$ in $10^{3} \mathrm{sec}$ at worst, was reduced by the feedback circuit to less than $0.05 \%$ in $10^{3} \mathrm{sec}$. Thus very small changes in EHD luminescence over times $\sim 10^{3}$ sec could be measured with confidence, as required in the study of the metastable behavior of the EHD-FE system.

Manipulation of the $1 \mathrm{amp}$ output ot create accurately reproducible cycles of excitation such as those shown in Fig. 4.1 was controlled by the reference function generator, Fig. 4.4a, and the timing logic, Fig. 4.5. The output $\mathrm{V}_{\mathrm{R}}$ of the function generator can be switched to any one of three DC levels, each independently adjustable from 0 to $1 \mathrm{~V}$, by logic signals L1 to L3; the time for which each level is enabled is controlled by switches SW1 to SW3 in Fig. 4.5 and can be independently varied from $1 \mathrm{sec}$ to $2048 \mathrm{sec}$. Normally signal LO is high and the reference voltage $V_{R}$ is grounded; an excitation pulse is created by successively switching $V_{R}$ to one or more levels. The order in which these levels are enabled is controlled by the mode switch, Fig. 4.5, and can be set in the seguences LO, L3, LO; or LO,L1,L3,LO; or LO,L1, L2, L3,LO. In al1 cases L1 corresponds to the large initial excitation 
for taking a "downgoing" data point, and L3 corresponds to the fina1 level at which the signal is recorded. Thus the sequence LO,L3, LO yields an "upgoing" (UP) point, and the sequence LO,L1,L3, LO yields a "downgoing" (DOWN) point as shown in Fig. 4.1. Steps in the output $V_{R}$, Fig. 4.4a, are smoothed by a buffered RC filter with selectable time constants of 1,3 , and $10 \mathrm{sec}$, so that the excitation pulse has a very long risetime as required, and $V_{R}$ changes exponentially from one level to the next. In order to ensure that the excitation decreases monotonical1y from L1 to L2 or L3, when the two current range switches, Fig. 4.4b, have different settings, $V_{R}$ can be preset to $\approx 150 \%$ of full scale by the $1 \mathrm{msec}$ pulse SR at the falling edge of LI.

The timing logic shown in Fig. 4.5 also controlled the operation of a Northern Scientific mode1 NS-905 multi-channe1 analyzer (MCA), which was used to integrate the luminescence signals. A list of the connections between the timing logic and the MCA is given in Table 4.1. At the beginning of each cycle, initiated by closing the "start" switch, the pulse $\overline{\mathrm{EP}}$ advances the MCA memory channel addressed. The excitation then cycles as described above; when level L 3 is reached, either $\widehat{\mathrm{EADD}}$ or $\overrightarrow{\mathrm{ESUB}}$ is enabled after a delay determined by the switch SW4, which allows the excitation to reach a constant leve1. These signals cause the MCA to respectively add or substract data into the memory channel addressed, integrating the signal as indicated in Fig. 4.1. Thus the data-taking procedure was very simple: in order to automatically cycle the excitation and record the signal it was only necessary to set the final excitation desired and depress the start button. 
and the total impurity concentration $N_{A}+N_{D}=N_{i}$ is found to be less than 10 times larger than $\left|N_{A}-N_{D}\right|$ from measurements of the impurity profiles along the length of a boule; thus the crystals are not highly compensated and the material is truly ultrapure. As discussed in chapter 2, neutral impurities can influence the nucleation of EHD by binding small multi-exciton complexes and droplets, lowering the potential barrier $\psi_{\mathrm{c}}$ to drop growth by the amount $\Delta \psi \approx \mathrm{B}$, the binding energy of a macroscopic drop to one impurity atom. Recently the value of $B$ has been calculated by several authors ${ }^{4.7,4.8}$ with the results $B \approx 5$ to $10 \mathrm{meV}$ for shallow donors. These results justify the estimate $\mathrm{B} \approx 4.3 \mathrm{meV}$ used in the calculations for inhomogeneous nucleation in chapter 3 .

Dislocations also have an important role in determining the properties of the Ge crystals used; some boules contain concentrations $\sim 10^{2} \mathrm{~cm}^{-2}$ to $\sim 10^{4} \mathrm{~cm}^{-2}$ of these defects whereas others are essentially dislocation-free. In dislocation-free Ge, lattice vacancies formed during growth precipitate into vacancy clusters and the resulting material always has $80 \mathrm{meV}$ deep p-type traps of concentration (2 to 4) $\times 10^{11} \mathrm{~cm}^{-3}$, believed to be associated with divacancy-hydrogen complexes. 4.9 Dislocations, if present in concentration $\gtrsim 10^{2} \mathrm{~cm}^{-2}$, attract and annihilate essentially all vacancies during the cooling process so that the resulting material is free of these deep traps. Therefore the influence of dislocations, vacancy clusters, and $80 \mathrm{meV}$ traps on EHD nucleation can be studied by examining both dislocated and dislocation-free Ge crystals. 


\section{$000040 \% 319$ \\ $-79-$}

Dislocations might be expected to most strongly influence droplet nucleation, because they produce relatively large regions of high strain in the crystal. Because the total energy of FE and EHD shifts downward under sufficiently large uniaxial stress, ${ }^{4 \cdot 10,4.11}$ these strained regions should attract and nucleate drops. The magnitude $\varepsilon$ of the strain field associated with a dislocation in Ge has the form

$$
\varepsilon=\mathrm{fa} / 2 \pi \mathrm{r}
$$

where $f$ is a complicated angular function of the order of magnitude 1 , $\mathrm{a}=0.566 \mathrm{~nm}$ is the lattice constant, and $\mathrm{r}$ is the radial distance from the dislocation. Because the minfmum uniaxial stress necessary to lower the EHD energy ${ }^{4.10}$ is $\approx 3 \times 10^{8}$ dyne $\mathrm{cm}^{-2}$, and the shear modulus of Ge is $\sim 10^{12}$ dyne $\mathrm{cm}^{-2}$, the energy of the e-h liquid about a dislocation can be reduced at distances $\mathrm{r} \lesssim 0.3 \mu \mathrm{m}$. This value is much 1arger than the critical radius $R_{c} \sim 0.03 \mu \mathrm{m}$ of EHD embryos. Furthermore, the binding energy per e-h pair of a drop on a dislocation can be greater than $\phi$, the condensation energy. Therefore, one expects dislocations to strongly shift the threshold for EHD formation and to dominate the process of nucleation. However, this effect is not observed, as discussed in chapter 5 . This evidence seems to indicate that the recombination rate at the dislocation core is large enough to destroy nascent EHD before they can grow to macroscopic size. Because dislocations in Ge are known ${ }^{4.12}$ to produce deep acceptor levels $\approx 0.19 \mathrm{eV}$ below the conduction band associated with dangling bonds in the core which act as recombination centers, this assumption seems reasonable. 
Vacancy clusters and the associated $80 \mathrm{meV}$ acceptor levels are expected to have less influence on EHD nucleation than dislocations. Because the strain field surrounding a vacancy cluster falls off as $(a / r)^{3}$ rather than $(a / r)$, the region in which the e-h liquid energy is lowered $(r \leqq 0.01 \mu \mathrm{m})$ is too small to contain more than one $\mathrm{e}-\mathrm{h}$ pair in the 1iquid. Thus the binding energy of an EHD on a vacancy cluster due to strain is expected to be of the order of a few meV, less than or comparable to that for an impurity atom. The EHD binding due to the associated $80 \mathrm{meV}$ acceptors is expected to be much smaller than for sha1low $(\approx 10 \mathrm{meV})$ impurities, because the spatial extent of the deeper level is smaller, and the acceptor cannot be as effectively screened by the e-h 1iquid.

The ultrapure Ge used is known to contain a number of other impurities $\mathrm{O}, \mathrm{Si}, \mathrm{H}$, and $\mathrm{Cu}$, none of which is measurably electrically active in the concentrations present (with the exception of the divacancy-hydrogen level already discussed). A11 of these impurities can conceavably interact with drops via the strain fields surrounding each impurity atom, but none would be expected to have as large an effect as a vacancy cluster for example. The upper limits to the concentrations of $\mathrm{O}, \mathrm{Si}, \mathrm{H}$, and $\mathrm{Cu}$ present are respectively $5 \times 10^{13} \mathrm{~cm}^{-3}$, $10^{16} \mathrm{~cm}^{-3}, 10^{14} \mathrm{~cm}^{-3}$, and $10^{12} \mathrm{~cm}^{-3}$. Hydrogen and Copper atoms are both relatively mobile in Ge and form complexes with acceptors and donors respectively or precipitate out of solution. Thus the $40 \mathrm{meV}$ acceptor level of $\mathrm{Cu}$, for example, is not observed in good crystals. 4.13 
A 1ist of all the Ge samples used in this work is given in Table 4.2, together with their characteristics. These samples were specially selected to test the effects of impurity type and concentration over the range $3 \times 10^{9} \mathrm{~cm}^{-3}$ to $4 \times 10^{12} \mathrm{~cm}^{-3}$ and the effect of dislocation density over the range 0 to $10^{4} \mathrm{~cm}^{-2}$. In all cases the sample number corresponds to the boule; the properties of the Ge (with the exception of $\tau_{x}$ ) were obtained from the records of $W$. L. Hansen at the Lawrence Berkeley Laboratory, and are identified by the LBL boule number and the position of the cut along the length of the boule. Most of Table 4.2 is self-explanatory, however a few comments are required. The Miller indices listed are those of the excited face of the sample. We have no systematic evidence that the orientation of the crysta1 affects the experimental results for nucleation phenomena. The dislocation density was measured by visually inspecting a speciallyetched face of the crystal and characterizing the density as low $\left(\sim 10^{2} \mathrm{~cm}^{-2}\right)$, medium $\left(\sim 10^{3} \mathrm{~cm}^{-3}\right)$ or high $\left(\sim 10^{4} \mathrm{~cm}^{-2}\right)$; thus these values are only accurate to the order of magnitude. One pair of samples, $150 \mathrm{D}$ and $150 \mathrm{~V}$, were cut $\sim 1 \mathrm{~cm}$ apart from a specially selected slice of Ge, half of which was dislocated and half was dislocation-free, in order to rigorously test the effect of dislocations on EHD nucleation. Finally, the values of $\tau_{x}$ listed were measured on the actual samples used by recording the decay of the FE luminescence at $4.2 \mathrm{~K}$. Those values marked a were obtained using chopped excitation, ${ }^{4.4}$ whereas those marked $b$ were obtained above EHD threshold using GaAs laser excitation, by measuring the FE decay after the EHD Iuminescence had disappeared. ${ }^{4.14}$ 
Because of the slow falling edge of the chopped excitation, the latter values are probably more accurate.

The samples were prepared in the following manner. First the sample was cut to the size $4 \times 9 \mathrm{~mm}^{2}$ from a slice of $\mathrm{Ge} \approx 3 \mathrm{~mm}$ thick with a diamond saw. Then the piece of Ge was polished by hand on a glass plate to an optical finish, starting with 1200 mesh abrasive to remove saw damage and finishing with $3 \mu \mathrm{m}$ diamond paste. The polished sample was then etched for $\gtrsim 60 \mathrm{sec}$ in CP-4 (see Ref. 4.12, for example) quenched immediately in analytic-reagent grade methanol, and washed in distilled water. Finally the samples were carefully inspected to ensure that the surface was smooth, we11-etched, and free of cracks or chips. This etching procedure produces a surface which is electrically very good; the value of the surface recombination velocity is nominally ${ }^{4.15} \mathrm{~S} \sim 10^{2} \mathrm{~cm} \mathrm{sec}^{-1}$ for free carriers. Assuming the same value of $\mathrm{S}$ for $\mathrm{FE}$, the fractional decrease in the steady-state $\mathrm{FE}$ concentration at the surface $4.16 \mathrm{~S} \tau_{\mathrm{x}} / \mathrm{L} \sim 10^{-2}$ is negligible, and we thus expect to be able to neglect the effects of surface recombination of $\mathrm{FE}$. The actual situation may be less ideal. Because each $F E$ is a bound e-h pair, an exciton need on1y find a center to absorb its crystal momentum in order to rapidly recombine. Comparison of EHD thresholds measured on the some sample with surface and volume excitation (see Chapter 5) indicates that the quantum efficiency for surface excitation is $\gtrsim 15 \%$. Mounting of the prepared samples was carefully done in a way, shown in Fig. 5.6, which avoided placing external stress or electrical contacts on the Ge crystals. Both of these mechanisms are known to destroy drops, and therefore interfere with the observation of 
metastable states of the EHD-FE system. The sample holder was a 0.060 inch thick $\mathrm{Cu}$ plate in which holes were cut $3.0 \mathrm{~mm}$ wide by $9.0 \mathrm{~mm}$ high as indicated in Fig. 5.6 to pass the exciting 1ight. The samples, typically $4 \times 3 \times 9 \mathrm{~mm}^{3}$, were mounted on the plate with gentle wire springs, and the Ge was mechanically and electrically insulated from both the plate and the springs by 0.010 inch thick mylar pads as shown in Fig. 4.6. This sample size and orientation (9 $\mathrm{mm}$ side vertical) was chosen so that 1) the minimum dimension was several FE diffusion lengths to avoid effects of the surfaces not excited, and 2) the vertical dimension was as large as the image of the spectrometer slit on the sample to maximize the amount of luminescence collected.

The mounted samples were immersed in liquid helium in an optica1 cryostat constructed at the University of California, Department of Physics. As shown in Figs. 4.1 and 4.6, exciting radiation passed through the front window of the cryostat and the hole in the sample holder, whereas the FE and EHD luminescence was collected from the opposite (back) face of the sample. The temperature of the liquid He bath was varied by pumping with a mechanical vacuum pump which could cool the bath to $\approx 1.5 \mathrm{~K}$, and when necessary with an additional large $33 \ell \sec ^{-1}$ mechanical pump yielding a minimum bath temperature $\approx 1.2 \mathrm{~K}$. The bath temperature was controlled by a large-throughput pressure regulator connected between the bath and the smaller pump, which reduced the temperature drift with time to less than $\sim 0.03 \mathrm{~K}$ per hour above $1.5 \mathrm{~K}$. The temperature was determined by measuring the He vapor pressure with either a $\mathrm{Hg}$ manometer or a Zimmerli gauge. 
Below the $\lambda$ point of liquid He, where most of the data were taken, sample heating due to the excitation is calculated to be $\lesssim 0.01 \mathrm{~K}$ for

the excitation levels used $\left(\mathrm{P}<10 \mathrm{~mW} \mathrm{~cm}{ }^{-2}\right)$ and can be neglected. Thus the temperature of the Ge samples could be accurately measured and controlled, as required for measurements of the metastable time development and the phase diagram of the EHD-FE system.

\section{Signal Detection Apparatus}

A special effort was made in the design of the detection apparatus to optimize sensitivity to the $709 \mathrm{meV}$ and $714 \mathrm{meV}$ luminescence of electron-hole drops and free excitons, in order to study the EHD-FE system near threshold at low temperatures, where the luminescence signals are very weak. The components which contribute most to the extremely high sensitivity of this apparatus are the ultra-sensitive Ge detector and the signal integrating electronics to be described below.

As shown in Fig. 4.1, FE and EHD 1uminescence was collected from the back face of the Ge samples by an aspheric plano-convex Pyrex lens of focal length $65 \mathrm{~mm}$ and diameter $72 \mathrm{~mm}$, and focussed onto the entrance slit of the spectrometer. A rotary mechanical chopper was used to modulate the signal at $380 \mathrm{~Hz}$ for phase sensitive detection, and two type 7-56 Corning glass filters (not shown) removed second and higher order spectra. The spectrometer was a $1 / 4 \mathrm{~m}$ Jarrel1-Ash f/3.5 instrument equipped with a 890 groove $\mathrm{mm}^{-1}$ grating blazed at the wavelength $1 \mathrm{\mu m}$, and with $1 \mathrm{~mm}$ wide entrance and exit slits, 


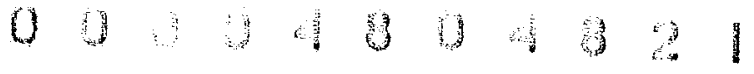 \\ $-85-$}

yielding a spectra1 resolution (FWHM) of $5.9 \mathrm{~nm}$. In order to record the intensities of the LA phonon replicas of the EHD and FE 1uminescence lines, the spectrometer was set at the wavelengths $1748.0 \mathrm{~nm}$ and $1734.0 \mathrm{~nm}$ respectively. With these settings and spectral resolution, the relative fractions of the $\mathrm{EHD}$ and $\mathrm{FE}$ luminescence lines transmitted by the spectrometer were $56.6 \%$ and $62.3 \%$ respectively, determined by convoluting the lineshapes ${ }^{4.17}$ with a triangular slit function of the correct width. Thus the recorded signals were proportional to the tota1 integrated intensities of the EHD and FE lines.

An intrinsic Ge particle detector was used as an exceptionally sensitive photodetector to record the EHD and FE I uminescence. The detector, fabricated by E. E. Haller at Lawrence Berkeley Laboratory, consisted of a $5 \times 10 \times 10 \mathrm{~mm}^{3}$ block of ultrapure Ge with diffused $\mathrm{Li}$ and evaporated $\mathrm{Cr}$ blocking contacts on the opposite $10 \times 10 \mathrm{~mm}^{2}$ faces. This detector was mounted in a variable-temperature, ultra-high vacuum, cold-finger dewar similar to those used for particle detectors with the exception of a Quartz window to pass the luminescence, which was focussed on a $5 \times 10 \mathrm{~mm}^{2}$ face. Between experiments the detector was stored at $\approx 100 \mathrm{~K}$, continuous $1 \mathrm{y}$ cooled by liquid nitrogen.

The success of this device as a photodetector at $\lambda \sim 1.75 \mu \mathrm{m}$ 1fes in the fact that the band gap of Ge decreases with temperature, so that the EHD Iuminescence, which 1 ies well below the gap at $4.2 \mathrm{~K}$, is indirectly absorbed at higher $\mathrm{T}$. Because the detector was made $10 \mathrm{~mm}$ thick, the absorption length of the luminescence need be only less than $\sim 1 \mathrm{~cm}$ in order to have acceptable quantum efficiency. Fortunately, 
this condition is satisfied for detector temperatures sufficiently low that the thermal generation rate of free carriers is not the primary source of noise. The detector temperature actually used during an experiment was $T=168 \pm 5 \mathrm{~K}$. Curves of the relative response of the detector over this temperature range are shown in Fig. 4.7; these data were obtained by illuminating the front slit of the spectrometer with a tungsten 1amp. Assuming 100\% efficiency for wavelengths shorter than $1.66 \mu \mathrm{m}$, the quantum efficiency of the device at $166 \mathrm{~K}$ is $59 \%$ for $\mathrm{FE}$ and $48 \%$ for EHD luminescence as indicated in Fig. 4.7.

Therefore the absorption 1ength of this light in the Ge is $\sim 1 \mathrm{~cm}$, and the EHD and FE luminescence produces free carriers throughout much of the intrinsic region of the detector depleted by the applied reverse bias of 90V. These carriers are in turn swept to the contacts by the internal electrical field. Thus the current through the device is proportional to the incident photon flux. The response time of this detector is limited by the collection time of the free carriers and can be quite fast. Assuming an internal field of $50 \mathrm{~V} \mathrm{~cm}^{-1}$, the transit time across the $5 \mathrm{~mm}$ width of the device given by the carrier mobility $\mu \sim 10^{4} \mathrm{~cm}^{2} \mathrm{~V}^{-1} \mathrm{sec}^{-1}$ at $170 \mathrm{~K}$ is $\sim 1 \mu \mathrm{sec}$. This value can be reduced to $\sim 200 \mathrm{nsec}$ by increasing the applied bias to 300V. However, for shorter wavelengths which are surface-absorbed the response time is much longer, $\sim 1$ msec, probably because the denser e-h plasma screens the collecting internal electrical field.

The signal from the detector was DC coupled to the sensitive current amplifier shown in Fig. 4.8, designed by Jan DeVries of the 


\section{$00+690482$}

Space Sciences Laboratory. The input FET of this circuit was specially selected for low noise, and the entire amplifier was encased in a mu-metal shield to reduce magnetic pick-up. The gain and speed of this amplifier are controlled by the feedback resistance and capacitance; thus the 100 Megohm resistor used with its residual capacity of $\sim 0.5 \mathrm{pf}$ gave a gain of $10^{8} \mathrm{VA}^{-1}$ and an $\mathrm{RC}$ response time $\sim 50 \mathrm{\mu sec}$.

The tota1 noise of the detector-preamplifier combination was measured under the conditions used during an experiment ( $90 \mathrm{~V} \mathrm{bias,} 380 \mathrm{~Hz}$ ) with a PAR-186A 1ock-in amplifier used as a noise meter connected to the output of the preamplifier. At the detector temperature 100k, the 1eakage current measured by a Kiethley model $160 \mathrm{~B}$ multimeter was $\approx 30 \mathrm{pA}$.

By dividing the noise voltage Indicated on the PAR $186 \mathrm{~A}$ by $10^{8} \mathrm{VA}^{-1}$, the preamplier gain, we obtained the rms noise referred to the input $i_{\mathrm{n}} \approx 40 \mathrm{fA} \mathrm{Hz}{ }^{-1 / 2}$, due to the amplifier alone $\left(1 \mathrm{fA}=10^{-15} \mathrm{~A}\right)$. At the operating temperature $168 \mathrm{~K}$, the leakage current increased to $4.6 \mathrm{nA}$ and the rms noise to $i_{\mathrm{n}} \approx 50 \mathrm{fA} \mathrm{Hz}-1 / 2$. This increase in total noise is consistent with the shot noise $39 \mathrm{fA} \mathrm{Hz}^{-1 / 2}$ expected from 4.6 nA. Thus we conclude that the system is primarily amplifier noise 1imited for detector temperatures $\leqslant 170 \mathrm{~K}$, but is dominated by leakage current noise at higher $\mathrm{T}$.

Values for the noise-equivalent power (NEP) and the detectivity $D^{*}$, which characterize this device, can be calculated from the measured value of $i_{n}$ above. The noise power is given by $N E P=i_{n} h \nu / 2 \eta=4 \times 10^{-14} \mathrm{WHz}^{-1 / 2}$ where $\mathrm{h} \nu=0.71 \mathrm{eV}$ is the incident photon energy in electron-volts and $n \approx 0.5$ is the quantum efficiency. 
The detectivity is given by

$$
\mathrm{D}^{*}=\mathrm{A}^{1 / 2} / \mathrm{NEP} \approx 2 \times 10^{13} \mathrm{~cm} \mathrm{~W}^{-1} \mathrm{~Hz}^{1 / 2}
$$

where $A \cong 0.5 \mathrm{~cm}^{2}$ is the active area of the device. This value of $D^{*}$ is more than two orders of magnitude greater than that for commercial cooled $\mathrm{PbS}$ detectors commonly used to study EHD luminescence. Thus this detector allows the measurement of luminescence signals which cannot be observed with more conventional devices, and is an essential part of the apparatus.

The signal from the preamplifier was further conditioned by the selective clipper shown in Fig. 4.9 to remove noise spikes due to cosmic rays absorbed by the detector. This circuit normally has an inverting voltage gain of 44 provided by the 733 video amplifier. When a fast risetime $(<10 \mu \mathrm{sec})$ pulse of voltage greater than the threshold value (typically $15 \mathrm{mV}$ ) occurs, the 711 comparator fires, turning on the FET, and shorting the output for $\approx 200 \mu \mathrm{sec}$. Thus fast spikes are removed without affecting the chopped luminescence signal. Although the improvement in signal to noise ratio was only marginal, this device was included in the apparatus.

The remaining electronics shown in Fig. 4.1 form a signal averaging system capable of directly integrating the luminescence signal for very long times to achieve high sensitivity. This system is based on a Northern Scientific NS-905 multi-channel analyzer (MCA) operated in the multi-channel scaler (MCS) mode, with a memory capacity of 512 channels. The signal from the selective clipper is first 


\section{$00034844 m 23$}

synchronously detected by a PAR corp. HR-8 lock-in amplifier set for a time constant of $0.1 \mathrm{sec}$. The output of the lock-in amplifier is connected to a Vidar voltage-to-frequency converter with a full-scale output of $100 \mathrm{kHz}$. The frequency of the resulting pulse train is then divided by 100 by two 7490 TTL integrated circuits and connected to the MCS input of the MCA. In order to integrate and store the signal for a given data point, the MCA is gated for a fixed time (24 sec) by the signal $\overline{\mathrm{EADD}}$ from the timing logic as shown in Figs. 4.1 and 4.5 and described in section 41. During this time pulses at the MCS input are added into the memory channel addressed, so that the final count in that channel is proportional to the integral of the signal. The timing logic also advances the channel addressed so successive data points are stored in successive channels. When a11 512 channe1s have been used, the contents of the memory can be read out on a teletype machine. Thus this system can automatically digitize, integrate, and store the data from an entire experiment. Because the integration times used were so long, the amount of data which can be taken during one day is severely limited, and automation and direct integration of the signal are very important. If the lock-in amplifier were used alone, set for the time constant $24 \mathrm{sec}$, the setting time to $1 \%$ accuracy, $120 \mathrm{sec}$, would reduce the total number of data points which could recorded by roughly a factor of four. 
CHAPTER 5. EXPERIMENTAL RESULTS AND ANALYSIS

In this chapter we present the results of a thorough experimental investigation of EHD nucleation phenomena in ultrapure Ge. The luminescence emitted by electron-hole drops and free excitons in optically excited Ge was used as a sensitive measure of the relative concentrations of these species. The study of the variation of the EHD and FE luminescence intensities vs. excitation, history of excitation, time, and temperature yields a great deal of information about the FE-EHD system: Section 51, basic hysteresis curves for $\mathrm{FE}$ and EHD at various temperatures which exhibit well-defined thresholds for EHD formation and breakup, and measurement of the EHD radiative enhancement factor $\rho$; section 52 , the metastable time development of the EHD signal; section 53, variation of excitation thresholds vs. impurity type and concentration, and dislocation density, and measurement of the EHD surface tension $\sigma$ from hysteresis curves; and section 54, detailed analysis of the FE-EHD phase diagram between $1.2 \mathrm{~K}$ and $2.0 \mathrm{~K}$ yield accurate measurements of $\sigma$ and the condensation energy $\phi$, and an estimate of the temperature-independent evaporation rate of EHD.

As described in chapter 4, the FE and EHD luminescence was spectrally selected by a spectrometer equipped with broad slits for high sensitivity. For the excitation and collection geometry used, (see chapter 4) the 1uminescence intensities $I_{d}$ and $I_{x}$ of $E H D$ and $F E$ respectively are given by 


$$
\begin{aligned}
& 00+0430452 \cdot 4 \\
& -91- \\
& \begin{array}{l}
I_{d}=C\left(f_{d} / \tau_{0}^{r}\right) \int_{0}^{d} N \nu d x \\
I_{x}=C\left(f_{x} / \tau_{x}^{r}\right) \int_{0}^{d} n d x
\end{array}
\end{aligned}
$$

where the constant of proportionality $\mathrm{C}$ is the same for both cases. Here $\mathrm{N}$ is the drop concentration, $\nu$ is the number of $\mathrm{e}-\mathrm{h}$ pairs contained in one drop, $\mathrm{n}$ is the $\mathrm{FE}$ density, $\mathrm{x}$ is the distance into the Ge sample from the excited surface, $\tau_{0}^{r}$ and $\tau_{x}^{r}$ are the radiative lifetimes for EHD and $F E$, and $f_{d}$ and $f_{x}$ are the relative detection effictencies for EHD and FE including the slit function of the spectrometer and the detector response (see chapter 4). In short, the signals $I_{d}$ and $I_{x}$ are proportional to the integrals with respect to depth into the sample of the total concentrations $N V$ and $n$ of e-h pairs condensed into EHD and $\mathrm{FE}$. The variation of $\mathrm{N} v$ and $\mathrm{n}$ in space is expected to be onedimensional, because the entire front surface of the samples is uniformly excited. In all cases, the signals $I_{d}$ and $I_{x}$ refer to the LA-phonon replica of the EHD and FE Iuminescence. This replica was used because it is the strongest and is allowed by selection rules for phonon emission, with no expected temperature dependence.

\section{Hysteresis Curves for Electron-Hole Drops and Free Excitons}

When the EHD luminescence signal $I_{d}$ is recorded while the excitation $P$ is continuously swept from zero to a maximum value $P_{\text {max }}$, then back to zero, a hysteresis curve is generated: the signal $\mathrm{I}_{\mathrm{d}}$ on the downgoing branch is larger than that on the upgoing branch, and the 
thresholds for the creation and breakup of EHD differ substantially. This effect was discovered by Lo et al. ${ }^{5.1}$ using this method of excitation. These authors noted irreproducibility in the results obtained for $I_{d}$. In order to avoid apparent1y irreproducible effects of the metastable time development of the EHD signal, and to ease comparison with nucleation theory, a11 of the data presented in this thesis was taken point-by-point as described in chapter 4. Upgoing data points were each taken by increasing the excitation from zero to a constant value $\mathrm{P}$ at which the upgoing EHD and $\mathrm{FE}$ signals $\mathrm{I}_{\mathrm{d}}^{+}$and $\mathrm{I}_{\mathrm{x}}^{+}$ were recorded; downgoing points were each taken by initially increasing the excitation from zero to a large value $\mathrm{P}_{\max }$, then decreasing the excitation to a constant value $\mathrm{P}$ at which the downgoing $\mathrm{EHD}$ and $\mathrm{FE}$ signals $\mathrm{I}_{\mathrm{d}}^{-}$and $\mathrm{I}_{\mathrm{X}}^{-}$were recorded.

Hysteresis curves for $\mathrm{EHD}$ and $\mathrm{FE}$ in $\mathrm{Ge}$ at $2.1 \mathrm{~K}$ recorded in this manner are shown in Fig. 5.1. These data were taken using surface excitation (see chapter 4) in order to obtain a wide range of excitation power. A large hysteresis is evident in both the EHD and FE signa1s $I_{d}$ and $I_{x}$ shown in Fig. 5.1. Drops created by the large initial excitation $\mathrm{P}_{\max }=0.9 \mathrm{~W} \mathrm{~cm}^{-2}$ in the downgoing case, remain as the excitation is reduced and cause the separation between the curves for $\mathrm{I}_{\mathrm{d}}^{-}$and $\mathrm{I}_{\mathrm{d}}^{+}$. These signals display sharp, separate excitation thresholds for the creation and destruction of $\mathrm{EHD}$ at $\mathrm{P}_{+} \approx 20 \mathrm{~mW} \mathrm{~cm}^{-2}$ and at $\mathrm{P}_{-} \approx 5 \mathrm{~mW} \mathrm{~cm}^{-2}$ respectively, which differ by a factor of four. Far above threshold the EHD signals $\mathrm{I}_{\mathrm{d}}{ }^{+}$and $\mathrm{I}_{\mathrm{d}}{ }^{-}$join and vary linearly with excitation $P$ as expected, because the majority of carriers created 
enter the EHD phase. Below threshold, the $\mathrm{FE}$ signals $\mathrm{I}_{\mathrm{x}}{ }^{+}$and $\mathrm{I}_{\mathrm{x}}{ }^{-}$do not display hysteresis and increase linearly with excitation as one expects for a simple system composed only of free excitons. At the respective thresholds $\mathrm{P}_{-}$and $\mathrm{P}_{+}$the FE signals $\mathrm{I}_{\mathrm{x}}{ }^{-}$and $\mathrm{I}_{\mathrm{x}}{ }^{+}$break downward from their linear dependence on $\mathrm{P}$ as an increasing fraction of excited e-h pairs enter the EHD phase. As a result, the FE signals display an inverted hysteresis which is consistent with the conservation of excited carriers as descrịbed in chapter 3 by Eq. (3.1). Thus the hysteresis curves of Fig. 5.1 exhibit large supersaturation effects analogous to those found for ordinary liquids such as water. All of the basic hysteresis phenomena for EHD and FE described above for Fig. 5.1 were observed for every sample studied at this temperature for both surface and volume excitation and are truly characteristic of the EHD-FE system in ultrapure Ge. These phenomena are in good qualitative agreement with the predictions of nucleation theory (see chapters 2 and 3); we present a detailed analysis in the following paragraphs.

An interesting feature of Fig. 5.1 not discussed further below, is the disappearance of hysteresis in $I_{X}$ and $I_{d}$ for excitation powers larger than $\mathrm{P} \approx 0.1 \mathrm{~W} \mathrm{~cm}^{-2}$; this phenomenon occurred at large $\mathrm{P}$ for a11 samples studied with surface excitation. Conceivably this effect could be caused by sample heating. However the estimated temperature rise for $P=0.1 \mathrm{~W} \mathrm{~cm}^{-2}$ is only $\sim 0.1 \mathrm{~K}$, too small a change to be effective. A more likely explanation is that phonons created by the surface excitation exert a sufficiently large force on EHD (the so-called phonon wind ${ }^{5.2}$ ) to move them away from the excited surface into the 
interior of the crystal where they disappear, thus destroying the memory of the EHD-FE system. Using the experimental estimate of Bagaev et a1., 5.2 we find that the force exerted on a drop of $3 \mu \mathrm{m}$ radius (see chapter 3 )

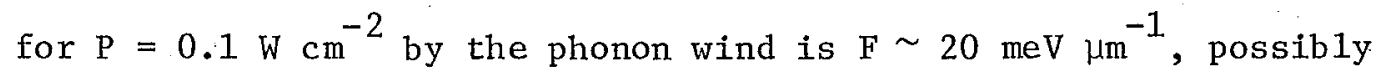
large enough to dislodge the drop from an impurity to which it is bound by the energy $\sim 10 \mathrm{meV} .^{5.3}$

A linear plot of the hysteresis curves for EHD and FE near threshold at $1.82 \mathrm{~K}$ is shown Fig. 5.2. This data and that shown in the following figures of this section were taken using volume excitation (see chapter 4). Compared to the original hysteresis data of Lo et al., ${ }^{5.1}$ these results are greatly improved in that they exhibit much higher sensitivity and are strictly reproducible. The curves in Fig. 5.2 clearly show all of the basic hysteretic phenomena described for Fig. 5.1 above. The EHD signal $I_{d}$ exhibits a large hysteresis, and sharp thresholds at $\mathrm{P}_{+} \approx 0.52 \mathrm{~mW} \mathrm{~cm}{ }^{-2}$ and $\mathrm{P}_{-} \approx 0.37 \mathrm{~mW} \mathrm{~cm}^{-2}$ as indicated in Fig. 5.2 for the creation and destruction of EHD. The FE signals $\mathrm{I}_{\mathrm{x}}^{+}$and $\mathrm{I}_{\mathrm{x}}^{-}$vary linearly with $\mathrm{P}$ for low excitation and break downward at the respective thresholds $\mathrm{P}_{+}$and $\mathrm{P}_{-}$, yielding an inverted hysteresis. These features are consistent with the condensation of $\mathrm{FE}$ into e-h drops as described in chapters 2 and 3. In fact, these data obey the conservation equation $P \propto I_{x}+a I_{d}$ for excited electrons and holes obtained from Eq. (3.1) in chapter 3, indicating that FE and EHD are the only important species present in the system. A least squares fit of this expression to the data of Fig. 5.2 yields a $\approx 4.6 \times 10^{-2}$, a value which is smaller than unity 


\section{$00+9480486$}

partially because the FE 1ifetime $\tau_{X}$ is much smaller than that for EHD (see Eq. 3.1).

Before proceeding further, we can use hysteresis data such as that shown in Figs. 5.1 and 5.2 to determine the radiative enhancement factor $\rho$ of the electron-hole liquid. This quantity is defined as the ratio of the electron density at the position of a hole to the average Iiquid density $\mathrm{n}_{\mathrm{o}}$ and is a sensitive measure of the degree of electronhole correlation in the liquid. Therefore, an experimental determination of $\rho$ is of considerable importance in assessing the validity of manybody theory used to calculate the properties of the EHL. We measure $\rho$ by extracting the ratio $\tau_{x}^{r} / \tau_{0}^{r}$ of the radiative lifetimes for $\mathrm{FE}$ and EHD from hysteresis data as described below.

The only assumption necessary to find the ratio $\tau_{x}^{r} / \tau_{0}^{r}$ is that the conservation equation, Eq. (3.1), holds for both branches of the hysteresis curves for $\mathrm{FE}$ and EHD at a given excitation level P; no nucleation theory is involved in the calculation. Using Eqs. (5.1) and (5.2) for the EHD and FE signals at the same excitation power $P$, we find immediately

$$
\tau_{x}^{r} / \tau_{o}^{r}=\left(\tau_{x} / \tau_{0}\right)\left(f_{x} / f_{d}\right)\left(I_{d}{ }^{-}-I_{d}^{+}\right) /\left(I_{x}^{+}-I_{x}{ }^{-}\right)
$$

The measured total lifetimes $\tau_{\mathrm{x}}$ and $\tau_{\mathrm{O}}$, and the instrumental functions $f_{x}$ and $f_{d}$ for $F E$ and $E H D$ are given in chapter 4. From data taken on samples 145, 150D, and 150V (see Table 4.1) using surface excitation we find $\tau_{x}^{r} / \tau_{0}^{r}=4.7 \pm 1.7$. The values of $\tau_{x}^{r} / \tau_{0}^{r}$ obtained were roughly independent of excitation $P$ near threshold and of temperature in the 
range 1.8 to $2.2 \mathrm{~K}$, where the data were taken. The error given above for $\tau_{x}^{r} / \tau_{0}^{r}$ is due to variation between Ge samples, and is much larger than the noise in the signals $I_{d}$ and $I_{x}$. This value for the ratio $\tau_{x}^{r} / \tau_{0}^{r}$ is to be compared with the earlier estimates $\tau_{x}^{r} / \tau_{0}^{r}=4.4 \pm 1.1$ from decay data ${ }^{5.4}$ and $\tau_{x}^{r} / \tau_{0}^{r}=16$ from luminescence efficiency. 5.5 The value of $\tau_{x}^{r} / \tau_{0}^{r}$ above, measured at separate given levels of excitation, is comparable to the value obtained by fitting the conservation equation to an entire set of hysteresis curves at once. For example, the coefficient $a \approx 4.6 \times 10^{-2}$ from the volume excitation data of Fig. 5.2 above yields $\tau_{x}^{r} / \tau_{0}^{r} \approx 7.9$.

Given $\tau_{x}^{r} / \tau_{0}^{r}$, one can find the enhancement factor $\rho$ by assuming that the matrix elements for the radiative decay of $\mathrm{FE}$ and EHD are identical. Thus

$$
\rho=\left(|\psi(0)|^{2} / n_{0}\right)\left(\tau_{x}^{r} / \tau_{o}^{r}\right)
$$

where $|\psi(0)|^{2}=1 / \pi a_{x}^{3}$ is the magnitude of the exciton wavefunction at the origin in relative coordinates, with $a_{x}$ the Bohr radius. Using the most recent value ${ }^{5.6} a_{x}=11.4 \mathrm{~nm}$, we find for the enhancement factor

$$
\rho=4.4 \pm 1.6
$$

This measurement compares reasonably we11 with the theoretical value 5.7 $\rho=2.3$ for Ge. However, the large variation of the measured value of $\rho$ with sample casts doubt on the overall accuracy of this and other $5.4,5.5$ measurements of the enhancement factor to date. It seems likely that this variation is associated with effects of the unknown 
mechanism which determines the $F E$ total lifetime $\tau_{x}$, and that more accurate, reliable measurements of $\rho$ will be possible when this mechanism is understood.

We return now to the discussion of hysteresis curves. Nucleation theory predicts that the downgoing threshold $\mathrm{P}_{-}$is a unique function of the temperature and time of observation for a given sample, and is independent of the initial large excitation $\mathrm{P}_{\max }$ used to record the data. Hysteresis curves for EHD taken at $1.80 \mathrm{~K}$ are shown in Fig. 5.3 for several different values of the initial excitation $\mathrm{P}_{\max }$ in the downgoing case. As shown in Fig. 5.3, the threshold $\mathrm{P}_{-}=0.63 \mathrm{~mW} \mathrm{~cm}^{-2}$ for EHD breakup accurately reproduces as the initial level is increased from $\mathrm{P}_{\max }=2.52 \mathrm{~mW} \mathrm{~cm}^{-2}$, just above the upgoing threshold, to $\mathrm{P}_{\max }=4.72 \mathrm{~mW} \mathrm{~cm}^{-2}$, in agreement with theory. The curves shown in Fig. 5.3 also provide a good opportunity to discuss the semi-quantitative theoretical description of EHD nucleation given in Section 31. The upgoing branch $\mathrm{I}_{\mathrm{d}}^{+}$, Fig. 5.3a is expected to be characterized by an increasing concentration $\mathrm{N}$ of drops of nearly constant size $v$, where $v$ is the number of e-h pairs in a given drop, and the FE density above threshold should be locally fixed at the threshold value $\mathrm{n}_{+^{\circ}}$. Unfortunately, the EHD signal $\mathrm{I}_{\mathrm{d}}^{+}$is proportional to the product $\mathrm{N} v$, so the separate variation of these quantities cannot be tested. In the downgoing case as the final excitation $P$ is reduced below $\mathrm{P}_{\max }$, the drop concentration is expected to remain constant while the size $\nu$ and the FE density $n$ decrease. When $n$ locally reaches the threshold value $\mathrm{n}_{-}$, EHD begin to break up, and the concentration $\mathrm{N}$ is 
expected to decrease with $P$ while the size $\nu$ remains nearly constant. Note that the excitation level at which EHD locally begin to breakup is larger than the threshold value $P_{-}$and is proportional to $\mathrm{n}_{-} / \tau_{\mathrm{x}}+\mathrm{N}_{\mathrm{M}{ }_{-} / \tau_{0}}$, where $\mathrm{N}_{\mathrm{M}}$ is the drop concentration created by $\mathrm{P}_{\max }$. The signals $\mathrm{I}_{\mathrm{d}}{ }^{-}$in Figs. 5.3b to $5.3 \mathrm{~d}$ all display a break in slope at a critical value $P_{c}$ of the excitation near the downgoing threshold, indicated for Fig. 5.3b. Moreover, the difference $\left(\mathrm{P}_{c}{ }^{-\mathrm{P}_{-}}\right)$is proportional to the initial excitation $\mathrm{P}_{\max }$, as expected from the relation given above. No break in slope is observed for Fig. 5.2 simply because $\mathrm{P}_{\max }$ is relative1y much 1arger than for Fig. 5.3. These features suggest that EHD begin to breakup at the excitation $P_{c}$ in Fig. 5.3; we will test this assumption shortly.

Unfortunately, the detailed shape of the curves shown in Figs. 5.2 and 5.3 cannot be directly compared with the theoretical hysteresis curves of Chapter 3 (Fig. 3.1), because the latter assume spatially uniform excitation. The analysis for non-uniform excitation is quite complicated, and should include FE diffusion. However, because the absorption length $\ell \approx 1 \mathrm{~mm}$ for volume excitation is larger than the FE diffusion length $\mathrm{L} \approx 0.7 \mathrm{~mm}$, we can construct a simple model. neglecting FE diffusion in which the results of Chapter 3 hold locally. Using the nearly exact expressions Eqs. (3.6) and (3.11) for the drop concentration, the integral of Eq. (5.1) for the EHD signal is computed numerically for the generation rate $G(x)=G_{0} \exp (-x / l)$. The downgoing expression, Eq. (3.11), is inapplicable when $\mathrm{n}>\mathrm{n}_{-}$; in this case we obtain NV by solving the conservation equation $\mathrm{Eq}$. (3.1) 
with $\nu$ determined by the approximate expression Eq. (2.20) for the stable radius. The parameters used in the calculation are those of Table 3.1 corrected for the improved values obtained by fitting the phase diagram as described in Section 54.

Results of this procedure are shown in Fig. 5.4; the horizontal and vertical scales have been adjusted so that this figure can be directly compared with Fig. 5.3. Here the integral $n_{d}=\int N v d x$ from Eq. (5.1) is plotted vs. $G_{0}$ for values of the initial generation rate $G_{M}$ in the downgoing case chosen to correspond to the values of $P_{\max }$ in Figs. 5.3b to $5.3 \mathrm{~d}$. The overall agreement between the calculation Fig. 5.4 and the data Fig. 5.3 is very good, providing a confirmation of nucleation theory. In particular, the shape of the upgoing branch of the theoretical hysteresis curves is in excellent agreement with that of the EHD signa1 $\mathrm{I}_{\mathrm{d}}^{+}$in Fig. 5.3a, and the ratios of the calculated and experimental thresholds $G_{+} / G_{-}=2.9$ and $P_{+} / P_{-}=2.6$ are nearly the same. The primary differences between theory and experiment are found in the downgoing curves: the calculated curves $\mathrm{n}_{\mathrm{d}}$ in Figs. $5.4 \mathrm{~b}$ to $5.4 \mathrm{~d}$ are relatively smaller than the EHD signals $\mathrm{I}_{\mathrm{d}}{ }^{-}$in Figs. $5.3 \mathrm{~b}$ to $5.3 \mathrm{~d}$, and they do not exhibit breaks in slope near threshold. These differences are consistent with the existence of a region of quasi-uniform excitation within the Ge crystal created by FE diffusion, as discussed in Chapter 4.

The theoretical prediction that the drop concentration $N$ remains locally constant along the downgoing branch of hysteresis curves for excitation leve1s $\mathrm{P}>\mathrm{P}_{\mathrm{c}}$ can be experimentally tested by using a 
specially constructed cycle of excitation. From the data of Fig. 5.3, we expect $\mathrm{N}$ to vary slowly with $\mathrm{P}$ for $\mathrm{P}>\mathrm{P}_{\mathrm{c}}$, but to decrease rapidly as the excitation falls below $\mathrm{P}_{c}$, reaching zero at $\mathrm{P}_{-} \cdot$ Data taken under the same experimental conditions as Fig. 5.3d, but with an additional step in the downgoing excitation cycle, are shown in Fig. 5.5. In recording this data, the initial level $\mathrm{P}_{\max }$ was the same as for Fig. 5.3d, and the final level $P$ at which the EHD signal was recorded was fixed at the constant value $\mathrm{P}=1.42 \mathrm{~mW} \mathrm{~cm}^{-2}$, just below the formation threshold $P_{+}$(see Fig. 5.3a). As shown in the insert to Fig. 5.5, only the additional excitation level $\mathrm{P}_{2}$ was varied, from zero to the value of $P$. Thus, if the drop concentration remains constant during the dip in excitation to the level $P_{2}$, the final signal $I_{d}$ shown in Fig. 5.5 will be independent of $\mathrm{P}_{2}$; if $\mathrm{N}$ decreases $\mathrm{I}_{\mathrm{d}}$ will also decrease. As indicated in Fig. 5.5, the recorded luminescence signal $I_{d}$ is nearly constant for $P_{2}>P_{c}$, indicating that $N$ changes very little as expected. For $\mathrm{P}_{2}<\mathrm{P}_{\mathrm{c}}$ the EHD signal decreases sharply with $\mathrm{P}_{2}$, and reaches zero at the destruction threshold $\mathrm{P}_{-}$, indicating that the number of EHD also decreases with $\mathrm{P}_{2}$ to zero at $\mathrm{P}_{-}$. The values of $P_{c}$ and $P_{-}$shown in Fig. 5.5 accurately agree with those of Fig. 5.3d. Thus "motion" along a given downgoing hysteresis curve such as Figs. $5.3 b$ to $5.3 d$ is reversible for excitation levels between $P_{c}$ and $\mathrm{P}_{\text {max }}$, but becomes irreversible for levels lower than $\mathrm{P}_{c}$, because the number of EHD decreases. The division of each downgoing curve into a range of excitation where $\mathrm{N}$ remains constant and a range in which $\mathrm{N}$ decreases for volume excitation strongly suggests that a region of 
quasi-uniform excitation is created within the crystal by FE diffusion as mentioned above; thus the detailed shape of Figs. 5.3 and 5.5 and the values of $\mathrm{P}_{\mathrm{c}}$ are determined by geometrical effects and cannot be directly compared with theory.

The hysteresis curves shown in Figs. 5.1 to 5.3 were all taken at temperatures $\mathrm{T} \approx 2 \mathrm{~K}$. For ordinary liquids such as water metastable supersaturation effects become larger as the temperature is reduced. However, for EHD in Ge at temperatures below $2 \mathrm{~K}$ the opposite is the case. A series of hysteresis curves for EHD taken on the same sample (315) at various temperatures between $1.9 \mathrm{~K}$ and $1.2 \mathrm{~K}$ is shown in Figs. 5.6 through 5.9. As $\mathrm{T}$ decreases from $1.86 \mathrm{~K}$ in Fig. 5.6 to 1.25K in Fig. 5.9, the thresholds $\mathrm{P}_{+}$and $\mathrm{P}_{-}$for the creation and destruction of EHD approach each other; at $1.25 \mathrm{~K}$ hysteresis in the EHD signal is entirely absent. As discussed in Chapters 2 and 3 this effect is expected from EHD nucleation theory and is a direct consequence of the finite lifetime of the e-h liquid. The temperature at which hysteresis dissappears, $T \approx 1.3$ to $1.4 \mathrm{~K}$ from Figs. 5.8 and 5.9 , is in excellent agreement with the theoretical value $\mathrm{T}=1.3$ to $1.4 \mathrm{~K}$ from Fig. 3.3.

Another novel effect predicted by EHD nucleation theory and caused by the EHD lifetime can be seen by examining the thresholds

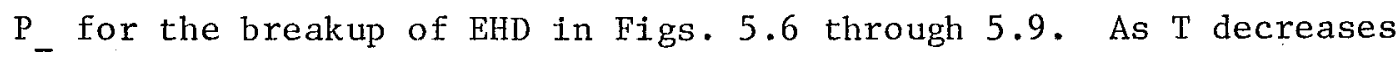
from $1.86 \mathrm{~K}$ to $1.25 \mathrm{~K}, \mathrm{P}_{\text {_ decreases from } 0.70 \mathrm{~mW} \mathrm{~cm}}^{-2}$ to $0.22 \mathrm{~mW} \mathrm{~cm}^{-2}$, only a factor of 3.2 . If $P_{\text {_ }}$ were proportional to the saturated vapor density, as is the case for ordinary liquids, one would expect $P$ _ to 
decrease by a factor $\approx 800$ for the same change in $\mathrm{T}$. Thus the measured thresholds for EHD breakup differ greatly from those predicted by the saturated $\mathrm{FE}$ density below $2 \mathrm{~K}$, and the threshold $\mathrm{P}_{\text {_ }}$ appears to approach a temperature-independent value at the lowest $T$ (see Figs. 5.8 and 5.9). These effects are in good agreement with EHD nucleation theory as shown in Fig. 3.3. The threshold ratios $P_{+} / P_{-}$obtained from Figs. 5.6 through 5.9 also agree well with the theoretical values from Fig. 3.3. A quantitative fit of nucleation theory to EHD threshold data is given and discussed in detail in Section 54.

As shown in Figs. 5.6 to 5.9 , the sensitivity of the apparatus is large enough that excitation thresholds $\mathrm{P}_{+}$and $\mathrm{P}_{-}$are clearly visible even at the lowest temperature measured, $1.25 \mathrm{~K}$, where $\mathrm{P}_{+}$is comparable to the illumination provided by room light. In order to extract we11-defined values for $\mathrm{P}_{+}$and $\mathrm{P}_{-}$from such data we develop approximate analytic expressions for integrated theoretical hysteresis curves such as Fig. 5.4, valid near threshold. Again we neglect FE diffusion and assume that the spatially uniform model of Chapters 2 and 3 is locally valid for volume excitation. For the spatially uniform generation of e-h pairs, we expect the EHD signals $\mathrm{I}_{d}^{+}$and $\mathrm{I}_{d}{ }^{-}$ to approach threshold linearly (see Eqs. 3.2 and 3.3), and the FE signals $\mathrm{I}_{\mathrm{x}}^{+}$and $\mathrm{I}_{\mathrm{x}}^{-}$just above threshold to be approximately constant, because the $\mathrm{FE}$ density is fixed at the threshold values. Using the simple linear expressions Eqs. (3.2) and (3.3) in the integral Eq. (5.1) for the EHD signal instead of the more accurate expressions used to calculate Fig. 5.4 we find 


$$
\begin{gathered}
0.4 .49439 \\
-103- \\
\mathrm{I}_{\mathrm{d}}^{ \pm} \approx \mathrm{C}\left(\mathrm{f}_{\mathrm{d}} / \tau_{\mathrm{o}}{ }^{\mathrm{r}}\right) \mathrm{G}_{ \pm} \tau_{\mathrm{o}} \ell\left[\left(\mathrm{G}_{\mathrm{o}} / \mathrm{G}_{ \pm}\right)-1-\ln \left(\mathrm{G}_{\mathrm{o}} / \mathrm{G}_{ \pm}\right)\right] .
\end{gathered}
$$

where $G(x)=G_{0} \exp (-x / l)$ is the volume generation rate. Note that all of the variation of $I_{d}$ with $G_{o}$ in Eq. (5.6) is contained in the expression within the square brackets. Near threshold the logarithm can be expanded yielding

$$
\mathrm{I}_{\mathrm{d}}^{ \pm} \approx \mathrm{C}\left(\mathrm{f}_{\mathrm{d}} / \tau_{\mathrm{o}}^{\mathrm{r}}\right) \mathrm{G}_{ \pm} \tau_{\mathrm{o}} \ell\left[\left(\mathrm{G}_{\mathrm{o}}-\mathrm{G}_{ \pm}\right)^{2} / 2 \mathrm{G}_{ \pm}^{2}\right]
$$

Thus the EHD signal $I_{d}$ is expected to increase quadratically with $P$ near threshold as a consequence of the excitation geometry. The variation of the $\mathrm{FE}$ signal $\mathrm{I}_{\mathrm{X}}$ just above threshold can be worked out within this model in a similar manner; we find from Eq. (5.2),

$$
\mathrm{I}_{\mathrm{x}}^{ \pm} \approx \mathrm{C}\left(\mathrm{f}_{\mathrm{x}} / \tau_{\mathrm{x}}^{\mathrm{r}}\right) \mathrm{G}_{ \pm}{ }^{\tau} \mathrm{x} /\left[\ln \left(\mathrm{G}_{\mathrm{o}} / \mathrm{G}_{ \pm}\right)+1\right]
$$

Again the variation of $I_{x}$ with $G_{0}$ is given by the expression in square brackets. From Eq. (5.8) we find that $I_{X}$ is expected to increase logarithmically with $\mathrm{P}$ above threshold with no break in slope at threshold. Inspection of Fig. 5.2 indicates that both the EHD and FE signals $I_{d}$ and $I_{x}$ vary with excitation $P$ qualitatively as predicted by Eqs. (5.7) and (5.8).

As illustrated in Fig. 5.10, the procedure used to extract excitation thresholds from hysteresis data was to plot the square root of the EHD signals $\mathrm{I}_{\mathrm{d}}{ }^{+}$and $\mathrm{I}_{\mathrm{d}}{ }^{-}$near threshold vs. $\mathrm{P}$ and linearly extrapolate $\mathrm{I}_{d}{ }^{1 / 2}$ to zero. The statistical error for the values $\mathrm{P}_{+}$and $\mathrm{P}_{-}$ obtained by this method was typica11y $<10 \%$. As shown in Fig. 5.10 
the signals $\mathrm{I}_{\mathrm{d}}{ }^{+}$and $\mathrm{I}_{\mathrm{d}}{ }^{-}$obey the quadratic expression $\mathrm{Eq}$. (5.7) quite we11, as do the data for other hysteresis curves taken with volume excitation. It seems likely that the cubic variation of $I_{d}$ with $P$ near threshold orginally observed by Pokravskii ${ }^{5.8}$ and used as evidence for a fixed drop concentration is an effect of the excitation geometry he used. The thresholds $\mathrm{P}_{+}$and $\mathrm{P}_{-}$obtained as described above are indicated for comparison in Figs. 5.6 to 5.9. This method of threshold extraction is used in section 54 to determine the EHD-FE phase diagram.

\section{Metastable Time Development}

The existence of hysteresis in the Iuminescence data of the preceeding section immediately indicates that the EHD-FE system can be in a metastable state for times as long as the $18 \mathrm{sec}$ delay used in recording the data. In this section we study the metastable time development of the EHD signals $\mathrm{I}_{\mathrm{d}}{ }^{+}$and $\mathrm{I}_{\mathrm{d}}{ }^{-}$for much longer delays as the system approaches equilibrium. Lo, Feldman and Jeffries ${ }^{5.1}$ originally found that the signals $\mathrm{I}_{\mathrm{d}}{ }^{+}$and $\mathrm{I}_{\mathrm{d}}{ }^{-}$approached each other slowly and that hysteresis could be maintained for times as long as $300 \mathrm{sec}$. However, they also noted the presence of jumps and irreproducibility in $I_{d}$, which we have found were caused by noise in the $\mathrm{Hg}$ arc lamp excitation used. We present data below which show that the EHD signals $\mathrm{I}_{\mathrm{d}}^{+}$and $\mathrm{I}_{\mathrm{d}}{ }^{-}$vary logarithmically with time as predicted by EHD nucleation theory in Chapter 3, and prove that metastable states of the EHD-FE system can actually exist for times as long as $6 \times 10^{4} \mathrm{sec}$. These conclusions were first presented in a preliminary account of this work. 5.9 
The method used to study the metastable time development of the EHD-FE system is described below. For either the upgoing or downgoing EHD signal $\mathrm{I}_{\mathrm{d}}^{+}$or $\mathrm{I}_{\mathrm{d}}{ }^{-}$, the excitation was cycled as described above in section 51. However, once the final leve1 $\mathrm{P}$ was reached, the excitation was held accurately constant by the feedback stabilization system (see Chapter 4) and the signal $I_{d}$ was integrated over successive $8 \mathrm{sec}$ intervals. In this way a plot of the signal $\mathrm{I}_{\mathrm{d}}{ }^{+}$or $\mathrm{I}_{\mathrm{d}}{ }^{-}$was obtained vs. the delay $\Delta t$ following the last change in excitation.

A plot of data obtained in this manner using volume excitation for sample 315 at $1.80 \mathrm{~K}$ is shown in Fig. 5.11. The excitation power $\mathrm{P} \approx 2.3 \mathrm{~mW} \mathrm{~cm}-2$ is the same for both curves in Fig. 5.11, and was chosen just above the threshold $P_{+}$for the creation of EHD (c.f. Fig. 5.3). The most striking feature of this data is that the system is still far from equilibrium after the delay $\Delta t=4 \times 10^{3} \mathrm{sec}$. Over this period of time the upgoing signal $\mathrm{I}_{\mathrm{d}}{ }^{+}$increases logarithmica11y with $\Delta t$ as shown in the lower portion of Fig. 5.11. This type of time variation is quite unusual in solid state physics, but is predicted by EHD nucleation theory as described in Chapter 3 . Thus the logarithmic variation of $\mathrm{I}_{\mathrm{d}}{ }^{+}$with $\Delta t$ in itself is evidence for nucleation phenomena. It is interesting to note that the apparent risetime ( $10 \%$ to $90 \%$ ) of the EHD signal $\mathrm{I}_{\mathrm{d}}^{+}$in Fig. 5.11 is extremely long, $\approx 1 \times 10^{3} \mathrm{sec}$. This value is $10^{7}$ times longer than that expected from the EHD lifetime $\tau_{0} \approx 40 \mu \mathrm{sec}$ alone and is dramatic evidence for EHD nucleation. The kinetics of EHD formation manifested in the luminescence signal on a much shorter time scale have been studied 
first in Ge by Staeh1: ${ }^{5.10}$ and later in Si by Shah et a1. ${ }^{5.11}$

The downgoing EHD signal $\mathrm{I}_{\mathrm{d}}^{-}$in Fig. 5.8 initially decreases logarithmically with $\Delta t$ but approaches the simple exponential dependence $I_{d} \propto \exp (-\Delta t / \tau)$ for $\Delta t>10^{3}$ sec with $\tau \approx 6 \times 10^{4} \mathrm{sec}$ as indicated by the solid curve. The initial logarithmic variation of $\mathrm{I}_{\mathrm{d}}^{-}$with $\Delta \mathrm{t}$ is evidence for nucleation as for the upgoing case, but the subsequent exponential decay is most likely due to an external mechanism which destroys EHD. Once individual drops are destroyed in the downgoing case, they are not replaced, and the drop concentration decreases exponentially for a constant rate of destruction. The surprising feature of this data is that the destruction time $\tau \approx 6 \times 10^{4} \mathrm{sec}$ for this sample is extremely long. Because EHD cannot be supplied with carriers if they move $\sim 1 \mathrm{~mm}$ into the crystal, this evidence indicates that individual drops are practically immobile under the conditions of this experiment. Recent measurements ${ }^{5.12}$ of the time development of the spatial distribution of EHD near threshold prove that drops must be pinned to crystal imperfections for low levels of excitation and place the upper limit $\mathrm{D}_{\mathrm{d}} \lesssim 10^{-7} \mathrm{~cm}^{2} \mathrm{sec}^{-1}$ on the EHD diffusion constant. These new results are not in conflict with those of a number of experiments ${ }^{5.13}$ performed with relatively high levels of excitation in which EHD are shown to move rapidly through the crystal.

Now we consider the magnitude of the logarithmic slope $\mathrm{dI}_{\mathrm{d}} / \mathrm{d}(\log \Delta \mathrm{t})$ of curves for $\mathrm{I}_{\mathrm{d}}{ }^{+}$and $\mathrm{I}_{\mathrm{d}}{ }^{-}$such as those shown in Fig. 5.11. The comparison of this slope with the theory presented in Chapter 3 is greatly complicated by the spatial variation of the generation rate 


\section{$00 \times 4944932$}

$-107-$

$\mathrm{G}(\mathrm{x})$ for volume excitation and by the difficulty of relating the signal $I_{d}$ in $m V$ to the integral $n_{d}=\int_{0}^{d} d x N V$ from $E q$. (5.1) of the amount of e-h liquid present in the sample. We roughly estimate the 1atter by comparing the hysteresis curves and theory shown above in Figs. 5.3 and 5.4; thus we find $\mathrm{n}_{\mathrm{d}}$ (pairs $\mathrm{cm}^{-3}$ ) $\sim 10^{10} \mathrm{I}_{\mathrm{d}}(\mathrm{mV})$. In order to treat the spatial variation of $G(x)$, we use the simple model neglecting FE diffusion described for the calculation of Fig. 5.4, in which the theory of Chapter 3 applies 1ocally. The integral $\mathrm{n}_{\mathrm{d}}$ is then computed numerically for various times $\Delta t$ from the nearly exact expressions Eqs. (3.6) and (3.11) for the drop concentration, as described above. The results for the upgoing integral $\mathrm{n}_{\mathrm{d}}{ }^{+}$calculated in this way at $1.80 \mathrm{~K}$ for the value $G_{0}$ corresponding to $P$ in Fig. 5.8 vary nearly logarithmically with $\Delta t$ as expected, with the slope $\mathrm{dn}_{\mathrm{d}}{ }^{+} / \mathrm{d}(\log \Delta \mathrm{t})=$ $1.2 \times 10^{10} \mathrm{~cm}^{-2}$ per decade. Thus the calculated slope of the EHD signal is $\mathrm{dI}_{\mathrm{d}}{ }^{+} / \mathrm{d}(\log \Delta \mathrm{t}) \sim 1 \mathrm{mV}$ per decade, which agrees very well with the value $2 \mathrm{mV}$ per decade measured from Fig. 5.11, providing further evidence for the validity of nucleation theory. However, the downgoing slope $\mathrm{dI}_{\mathrm{d}}{ }^{-} / \mathrm{d}(\log \Delta t) \approx 0.5 \mathrm{mV}$ per decade measured from Fig. 5.11 for $\Delta t<10^{3}$ sec is orders of magnitude larger than the calculated value. The reason for this discrepancy is unclear at present. It seems likely that the slope of the calculated drop breakup rate $J_{-}$vs. n from Chapter 2 is unphysically steep; thus the value used for the coefficient

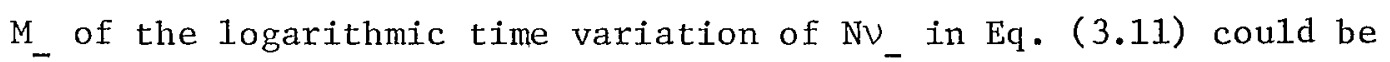
much too small. Another possible source of error is the neglect of FE diffusion in the above calculation. 
For different 1eve1s of excitation, the logarithmic slope of the EHD signals $\mathrm{I}_{\mathrm{d}}^{+}$and $\mathrm{I}_{\mathrm{d}}{ }^{-}$vs. $\Delta \mathrm{t}$ changes. At $1.80 \mathrm{~K}$ the upgoing slope increased to $\mathrm{dI}_{\mathrm{d}}^{+} / \mathrm{d}(\log \Delta t) \approx 5 \mathrm{mV}$ per decade from $2 \mathrm{mV}$ per decade as the excitation increased from $\mathrm{P}=2.13 \mathrm{~mW} \mathrm{~cm} \mathrm{~cm}^{-2}$ to $\mathrm{P}=2.90 \mathrm{~mW} \mathrm{~cm}^{-2}$. This increase is consistent with theory; the calculated slope in this case is proportional to the volume of the sample occupied by EHD (see Chapter 3), which increases with excitation power. The downgoing slope measured at $1.80 \mathrm{~K}$ increased to $\mathrm{dI}_{\mathrm{d}}^{-} / \mathrm{d}(\log \Delta \mathrm{t}) \approx 1.1 \mathrm{mV}$ per decade as the excitation decreased to $\mathrm{P}=0.76 \mathrm{~mW} \mathrm{~cm}^{-2}$, very near the downgoing threshold. At this excitation the signal $\mathrm{I}_{\mathrm{d}}{ }^{-}$was observed to decrease logarithmically all the way to zero in $\Delta t \approx 1000 \mathrm{sec}$. Expressed as a fraction of the initial signal $\mathrm{I}_{\mathrm{d}}{ }^{-}$, the downgoing slope in this case increased by a factor of 20 as threshold was approached. This increase is qualitatively in accord with nucleation theory as discussed in Chapter 3 .

Plots of the signals $\mathrm{I}_{\mathrm{d}}^{+}$and $\mathrm{I}_{\mathrm{d}}{ }^{-}$vs $\log \Delta \mathrm{t}$ for the same sample (315) at $1.50 \mathrm{~K}$ are shown in Fig. 5.12. As for Fig. 5.11, the excitation $\mathrm{P}=0.55 \mathrm{~mW} \mathrm{~cm}^{-2}$ was chosen just above the upgoing threshold, and is the same for both curves. Like the data at 1.80K, both the EHD signals $\mathrm{I}_{\mathrm{d}}^{+}$and $\mathrm{I}_{\mathrm{d}}{ }^{-}$shown in Fig. 5.12 initially vary logarithmically with $\Delta t$ as predicted by nucleation theory. The slope $\mathrm{dI}_{\mathrm{d}}^{+} / \mathrm{d}(\log \Delta \mathrm{t}) \approx$ $0.8 \mathrm{mV}$ per decade for the upgoing curve in $\mathrm{Fig} .5 .12$ agrees reasonably well with the value $\mathrm{dI}_{\mathrm{d}}^{+} / \mathrm{d}(\log \Delta \mathrm{t}) \sim 0.4 \mathrm{mV}$ per decade estimated from the integral $\mathrm{dn}_{\mathrm{d}}^{+} / \mathrm{d}(\log \Delta \mathrm{t})=0.37 \times 10^{10} \mathrm{~cm}^{-2}$ per decade calculated as described above. As for the data at $1.80 \mathrm{~K}$, the downgoing slope 


\section{$00+44904333$}

$-109-$

$\mathrm{dI}_{\mathrm{d}}{ }^{-} / \mathrm{d}(\log \Delta \mathrm{t}) \approx 5 \mathrm{mV}$ per decade from Fig. 5.12 is several orders of magnitude larger than the calculated value. Thus the metastable time development of the EHD-FE system at $1.50 \mathrm{~K}$ is similar in some respects to that at $1.80 \mathrm{~K}$, and comparable agreement with theory is found.

A striking feature of Fig. 5.12 not present in the data at $1.80 \mathrm{~K}$ is that the EHD-FE system actually reaches equilibrium at $\Delta t \approx 100$ sec. As shown in Fig. 5.12, the signals $\mathrm{I}_{\mathrm{d}}{ }^{+}$and $\mathrm{I}_{\mathrm{d}}{ }^{-}$join at $\Delta \mathrm{t} \approx 100 \mathrm{sec}$, and the noise in both signals increases substantially, presumably because the system slowly fluctuates about equilibrium. The small downward slope of $\mathrm{I}_{\mathrm{d}}{ }^{+}$and $\mathrm{I}_{\mathrm{d}}{ }^{-}$for $\Delta t>100 \mathrm{sec}$ is compatible with that expected from the very sma11 upward drift $\Delta \mathrm{T} \sim 0.01 \mathrm{~K}$ in the measured temperature of the liquid He bath while the datawere taken. The fact that the equilibration time $\approx 10^{2} \mathrm{sec}$ from Fig. 5.12 is very much smaller that the time $\sim 10^{7} \mathrm{sec}$ at $1.50 \mathrm{~K}$ predicted by nucleation theory in Chapter 3, is a result of the relatively large slope of $\mathrm{I}_{\mathrm{d}}{ }^{-}$, not predicted by theory. It is interesting to note that the shorter equilibration times predicted by theory at lower temperatures are caused by a decrease in magnitude of the predicted hysteresis rather than an increase in the rate of change of the drop concentration. The calculated coefficients $M_{+}$and $M_{-}$(see Chapter 3) which determine these rates only change from $1.6 \times 10^{11} \mathrm{~cm}^{-3}$ to $3.7 \times 10^{10} \mathrm{~cm}^{-3}$ and from $1.2 \times 10^{9} \mathrm{~cm}^{-3}$ to $2.2 \times 10^{9} \mathrm{~cm}^{-3}$ respective1y as the temperature is decreased from $1.80 \mathrm{~K}$ to $1.50 \mathrm{~K}$.

The fact that hysteresis is present in the signals $\mathrm{I}_{\mathrm{d}}{ }^{+}$and $\mathrm{I}_{\mathrm{d}}{ }^{-}$ of Fig. 5.12 for times $\Delta t<100 \mathrm{sec}$, but not for times $\Delta t>100 \mathrm{sec}$ 
underscores the importance of recording both signals at a fixed time $\Delta t$ of observation if these data are to be compared with nucleation theory. The data of Fig. 5.12 also indicate indirectly that the measured values of the excitation thresholds $\mathrm{P}_{+}$and $\mathrm{P}_{-}$depend upon the time $\Delta t$. Direct evidence for this effect is given in Fig. 5.13, where $\mathrm{I}_{\mathrm{d}}{ }^{-}$vs. $\Delta t$ is plotted for the same sample (315) at $1.50 \mathrm{~K}$ but a lower level of excitation $\mathrm{P}=0.44 \mathrm{~mW} \mathrm{~cm}^{-2}$ very near the downgoing threshold $\mathrm{P}_{-}$. As shown in Fig. 5.13, the downgoing signal $\mathrm{I}_{\mathrm{d}}^{-}$decreases logarithmically, reaching zero at $\Delta t \approx 200 \mathrm{sec}$. Thus the excitation level used for Fig. 5.13 1ies above the downgoing threshold for $\Delta t<200 \mathrm{sec}$, but below this threshold for $\Delta t>200 \mathrm{sec}$. Variation of the thresholds for the formation and breakup of EHD with the time of observation is predicted by nucleation theory and discussed in Chapter 3 .

At temperatures below $\approx 1.3 \mathrm{~K}$, EHD nucleation theory predicts that hysteresis is entirely absent and that the EHD-FE system is in dynamic equilibrium. A plot of the signals $\mathrm{I}_{\mathrm{d}}{ }^{+}$and $\mathrm{I}_{\mathrm{d}}{ }^{-}$vs. $\Delta t$ at $1.25 \mathrm{~K}$ recorded on the same sample (315) as the data above is shown in Fig. 5.14. Again, the excitation power $\mathrm{P}=0.37 \mathrm{~mW} \mathrm{~cm}{ }^{-2}$ was chosen just above the threshold for the formation of drops. As shown in Fig. 5.14 the signals $\mathrm{I}_{\mathrm{d}}{ }^{+}$and $\mathrm{I}_{\mathrm{d}}{ }^{-}$at this temperature are equal as expected from the lack of hysteresis in the curves shown in Fig. 5.9 for 1.25K. More importantly, the signals $\mathrm{I}_{\mathrm{d}}^{+}$and $\mathrm{I}_{\mathrm{d}}{ }^{-}$in Fig. 5.14 are accurately constant for $\Delta t>10 \mathrm{sec}$, indicating that the equilibration time at $1.25 \mathrm{~K}$ is 1 ess than $10 \mathrm{sec}$, and proving that metastability is entirely absent for longer times. 


\section{Effect of Impurities and Dislocations on Nucleation; Surface Tension Measurement}

For ordinary liquids such as water, the process of droplet nucleation is dominated by contaminants and surfaces unless the vapor is carefully purified and held in a clean container. Various impurities can act as nucleation centers by binding nascent drops, lowering or destroying altogether the potential barrier to nucleation. Thus the observed supersaturation ratio $\mathrm{n}_{+} / \mathrm{n}_{\mathrm{s}}$ of the threshold vapor density $\mathrm{n}_{+}$ for drop formation in a dirty system can be much less than the homogeneous value. Also, the final concentration of nucleated drops of ordinary liquids is typically constant, equal to the density of nucleation centers.

In this section we present an experimental study of the influence of shallow impurities, dislocations, and vacancy clusters on the process of EHD nucleation in ultrapure Ge crystals. As discussed in detail in Section 42, these defects are those expected to be most active as nucleation centers in the crystals studied. Briefly, we find that dislocations are ineffective as nucleation centers, and that EHD most probably form on neutral shallow impurities. The theory for this type of inhomogeneous nucleation is presented in Chapters 2 and 3 . In contrast to the case for ordinary liquids, these impurities are expected to have only a small effect on the observed supersaturation ratio $\mathrm{n}_{+} / \mathrm{n}_{\mathrm{s}}$ for EHD formation. Also, the EHD concentration $\mathrm{N}$ is only a small fraction of the impurity concentration, and $\mathrm{N}$ is expected to increase with excitation in a manner qualitatively 
identical to that for homogeneous nucleation. In the latter part of this section we use the measured excitation threshold ratios $P_{+} / P_{-}$for different samples to determine the EHD surface tension $\sigma$ using nucleation theory as described in Section 34. The variation of the value of $\sigma$ with temperature is also presented for various types of EHD nucleation.

Unless stated otherwise the data presented in this section was recorded using an unstabilized tungsten lamp as a source of surface excitation; otherwise the apparatus and procedure used was that described in Chapter 4. As discussed in Section 41, volume excitation is preferred for quantitative hysteresis measurements. However, no major differences were found between the results of surface and volume excitation, and we believe that the conclusions of this section are accurate. Excitation thresholds $\mathrm{P}_{+}$and $\mathrm{P}_{-}$were extrapolated from linear plots of the EHD signals $\mathrm{I}_{\mathrm{d}}^{+}$and $\mathrm{I}_{\mathrm{d}}{ }^{-}$vs. P. The measured parameter used in this section to study the effect of crystal defects and to determine the EHD surface tension is the ratio $\mathrm{P}_{+} / \mathrm{P}_{-}$of the excitation thresholds for the creation and destruction of EHD at a given temperature. A careful analysis of the temperature variation of the calibrated FE density thresholds $n_{+}$and $n_{-}$for volume excitation is presented in Section 54 together with a more reliable measurement of $\sigma$ obtained by fitting the entire FE-EHD phase diagram at once. As discussed in Section 42, dislocations might be expected to be very effective nucleation centers for EHD in Ge. The large strain field surrounding a dislocation attracts free excitons and considerably 
lowers the energy per e-h pair of the e-h liquid. A detailed analysis of EHD nucleation on dislocations is quite difficult; qualitatively one expects a pronounced shift (by a factor $\sim 10^{2}$ ) of the threshold $\mathrm{P}_{+}$to lower excitation, because $\mathrm{FE}$ are attracted to regions of maximum strain, and a substantial change in the threshold ratio $\mathrm{P}_{+} / \mathrm{P}_{-}$.

The effect of dislocations on EHD nucleation was studied by measuring the thresholds $\mathrm{P}_{+}$and $\mathrm{P}_{-}$for eight crystals of ultrapure Ge. These samples were cut to the same size, and identically etched and mounted. Two samples were essentially undislocated with vacancy c1usters and associated $80 \mathrm{meV}$ acceptor leve1s present (see Section 42); the other six were free of vacancy clusters but were dislocated in the range $10^{2} \mathrm{~cm}^{-2}$ to $10^{4} \mathrm{~cm}^{-2}$. One pair of samples (150D and 150V) was cut $\sim 1 \mathrm{~cm}$ apart from the same slice of $\mathrm{Ge}$, in order to ensure common characteristics other than dislocations and vacancy clusters. The hysteresis curves for the EHD signals $\mathrm{I}_{\mathrm{d}}{ }^{+}$and $\mathrm{I}_{\mathrm{d}}{ }^{-}$vs. $\mathrm{P}$ measured on all eight samples at $\mathrm{T} \approx 2.1 \mathrm{~K}$ were qualitatively identical. No major shift in the excitation threshold $\mathrm{P}_{+}$with dislocation density was observed, either for surface or for volume excitation. For example, the thresholds for EHD formation measured with volume excitation on samples $150 \mathrm{D}$ and $150 \mathrm{~V}$ at $\mathrm{T}=2.0 \mathrm{~K}$ were respectively $\mathrm{P}_{+}=1.8 \mathrm{~mW} \mathrm{~cm}^{-2}$ and $\mathrm{P}_{+}=0.61 \mathrm{~mW} \mathrm{~cm}{ }^{-2}$; this difference is roughly comparable to that expected from the FE lifetime alone (see Table 4.1).

A plot of the measured threshold ratios $\mathrm{P}_{+} / \mathrm{P}_{-}$vs. dislocation density for all eight samples at $2.1 \mathrm{~K}$ is shown in Fig. 5.15. The sample numbers and the dominant impurity types are indicated in the 
figure. As shown in Fig. 5.15, the measured ratios $P_{+} / P_{-}$exhibit no significant correlation with either dislocation density or type of electrica1 impurity. The apparent scatter of the measured values for $\mathrm{P}_{+} / \mathrm{P}_{-}$is primarily caused by the variation of $\mathrm{P}_{+} / \mathrm{P}_{-}$with impurity concentration (see below). Using volume excitation the measured ratios for samples $150 \mathrm{D}$ and $150 \mathrm{~V}$ were respectively $\ln \left(\mathrm{P}_{+} / \mathrm{P}_{-}\right)=0.99$ and $\ln \left(\mathrm{P}_{+} / \mathrm{P}_{-}\right)=0.83$ at $\mathrm{T}=2.0 \mathrm{~K}$ confirming this conclusion. Thus neither the EHD hysteresis curves nor the excitation thresholds $\mathrm{P}_{+}$and $\mathrm{P}_{-}$ exhibited any significant variation with dislocation density in the range 0 to $10^{4} \mathrm{~cm}^{-2}$. Because dislocations are expected to be very effective nucleation centers, we conclude that the e-h recombination rate at the dislocation core must be sufficient to destroy nascent EHD nucleated on dislocations before they can grow to macroscopic size. That is, dislocations may nucleate EHD embryos, but these are so quickly destroyed that they do not contribute significantly to the luminescence signals $I_{d}$ observed. This conclusion also suggests that dislocations can be a significant center for the non-radiative recombination of $\mathrm{FE}$. Barring dislocations, shallow electrical impurities are expected to be most effective as nucleation centers in the crystals studied (see Section 42). Because the excited samples are continually flushed with free carriers, and the impurity ionization time at $2 \mathrm{~K}$ is extremely long, the vast majority of these impurities are expected to be electrically neutral. A replot of the data of Fig. 5.15 for the threshold ratio $\mathrm{P}_{+} / \mathrm{P}_{-}$vs. net impurity concentration $\left|\mathrm{N}_{A}-\mathrm{N}_{D}\right|$ is shown in Fig. 5.16; the data for the undislocated samples has been omitted 
because $\left|\mathrm{N}_{\mathrm{A}}-\mathrm{N}_{\mathrm{D}}\right|$ is controlled by $80 \mathrm{meV}$ deep levels in this case. As shown in Fig. 5.16, the measured threshold ratios $\mathrm{P}_{+} / \mathrm{P}_{-}$are significant1y correlated with impurity concentration; $\mathrm{P}_{+} / \mathrm{P}_{-}$decreases by roughly a factor of 1.5 as $\left|N_{A}-N_{D}\right|$ increases from $3 \times 10^{9} \mathrm{~cm}^{-3}$ to $4 \times 10^{12} \mathrm{~cm}^{-3}$. A similar but smallex decrease in the threshold ratio with total impurity concentration $\mathrm{N}_{i}=\mathrm{N}_{A}+\mathrm{N}_{\mathrm{D}}$ is predicted by inhomogeneous nucleation theory for neutral impurities as described in Chapter 2; the calculated values $\mathrm{P}_{+} / \mathrm{P}_{-}$decrease by approximately $15 \%$ as $\mathrm{N}_{i}$ increases from $10^{10} \mathrm{~cm}^{-3}$ to $10^{13} \mathrm{~cm}^{-3}$. The correlation of the measured values $\mathrm{P}_{+} / \mathrm{P}_{-}$ with impurity concentration is strong evidence that the nucleation of EHD actually observed takes place on shallow electrical impurities, at least for the more impure crystals studied.

The conclusion that EHD form on shallow impurity atoms is important to the analysis of threshold data in terms of nucleation theory, and thus the measurement of the EHD surface tension $\sigma$ and condensation energy $\phi$. Because the concentration of EHD is typically very small $\left(\sim 10^{7} \mathrm{~cm}^{-3}\right)$, an undetectable density of unusual defects could in principle dominate the process of nucleation. The identification of impurities as active nucleation centers in the samples studied rules out this possibility. Also, the calculated thresholds for homogeneous nucleation and inhomogeneous nucleation on impurity atoms (see Chapter 3) are quantitatively too similar to identify the type of nucleation by analysis of the data for on1y one sample. The similarity of these two cases is in marked contrast to the behavior of ordinary liquids as discussed above. Possibly the most important effect of impurity atoms 
is to stabilize small multi-exciton complexes which grow into macroscopic EHD.

As described in Chapter 3, we can use measurements of the excitation threshold ratio $\mathrm{P}_{+} / \mathrm{P}_{-}$such as those above to determine the EHD surface tension $\sigma$. The procedure, described in detail in Section 34, is to calculate $\sigma$ using EHD nucleation theory from the value of $\mathrm{P}_{+} / \mathrm{P}_{-}$ measured at a given temperature. The values used for the EHD parameters other than $\sigma$ are those given in Tab1e 3.1, and the temperatureindependent evaporation rate $c$ is not included in the calculation. Because $\mathrm{P}_{+} / \mathrm{P}_{-}$is a rapidly varying function of $\sigma$ (see Fig. 3.2), this method yields accurate results for the surface tension. Also, the temperature variation of the measured values of $\sigma$ can be studied, assuming different types of nucleation. However, this method has the disadvantage that the individual variation of $\mathrm{P}_{+}$and $\mathrm{P}_{-}$with $\mathrm{T}$ is not included in the analysis. An improved measurement of $\sigma$ obtained by fitting the entire measured FE-EHD phase diagram at once is presented in Section 54 .

Table 5.1 lists the threshold ratios $\mathrm{P}_{+} / \mathrm{P}_{-}$measured at $2.1 \mathrm{~K}$ for seven different samples of ultrapure Ge and the values of the surface tension $\sigma$ calculated from $\mathrm{P}_{+} / \mathrm{P}_{-}$for four different assumptions regarding the type of nucleation. Repeated measurements for samples 315, 393, and 391 demonstrate the reproducibility of the method. As shown in Table 5.1, the measured values of $\sigma$ repeat very well from sample to sample and depend surprisingly little upon the type of nucleation assumed in the analysis. As the net impurity concentration increases 
from $3 \times 10^{9} \mathrm{~cm}^{-3}$ for sample 315 to $4 \times 10^{12} \mathrm{~cm}^{-3}$ for sample 400 , the value for $\sigma$ deduced decreases slightly, by $\approx 20 \%$; no correlation is found with dislocation density. The values of $\sigma$ calculated assuming homogeneous nucleation and inhomogeneous nucleation on neutral impurities with the barrier lowering $\Delta \psi=50 \mathrm{~K}$ (see Chapter 2) are practically identical, as we expect from the similarity of the calculated thresholds in these two cases (see Fig. 3.3). As the value assumed for $\Delta \psi$ increases from $50 \mathrm{~K}$ to $200 \mathrm{~K}$, the surface tension calculated for inhomogeneous nucleation increases by $\approx 40 \%$. Using our result that EHD form an impurity atoms and the value $\Delta \psi=100 \mathrm{~K}$, which agrees with the recently calculated value ${ }^{5.3} \mathrm{~B} \approx 10 \mathrm{meV}$ for the binding energy of an EHD on a neutral donor, we average the results shown in Table 5.1 for this case to obtain

$$
\sigma=(2.6 \pm 0.2) \times 10^{-4} \mathrm{erg} \mathrm{cm}^{-2}
$$

at $2.1 \mathrm{~K}$, where the error shown is purely statistical. By simply averaging all of the results for $\sigma$ shown in Table 5.1, we find a similar value $\sigma=(2.6 \pm 0.4) \times 10^{-4} \mathrm{erg} \mathrm{cm}^{-2}$ at $2.1 \mathrm{~K}$. The systematic error for $\sigma$ introduced by uncertainties in the parameters used in the analysis is comparable to that shown in Eq. (5.9). Replacing the value $\phi=2.0 \mathrm{meV}$ at $\mathrm{T}=0 \mathrm{~K}$ used for the $\mathrm{EHD}$ condensation energy in the analysis for Eq. (5.9) by the recent spectroscopic measurement ${ }^{5.6} \phi=1.8 \mathrm{meV}$ at $\mathrm{T}=0 \mathrm{~K}$ we find the mean value $\sigma=2.4 \times 10^{-4} \mathrm{erg} \mathrm{cm}^{-2}$ at $2.1 \mathrm{~K}$. In general the surface tension (actually the surface free energy) of a liquid varies with temperature. Because the e-h liquid is a 
degenerate Fermi 1iquid we expect the form of this temperature dependence to be $\sigma=w\left(1-\delta_{\sigma} T^{2}\right)$ well below the critical temperature 5.14 $\mathrm{T}_{\mathrm{c}}=6.5 \mathrm{~K}$, where $\mathrm{w}$ is the surface energy at $\mathrm{T}=0$. Reinecke and Ying 5.15 have suggested that $\delta_{\sigma}=\mathrm{T}_{\mathrm{c}}^{-2}=2.4 \times 10^{-2} \mathrm{~K}^{-2}$; actually this value should be an upper limit to $\delta_{\sigma}$, because one expects significant higher order corrections to $\sigma$ near the critical point. The quantity calculated by theorists is the surface energy w rather than $\sigma$. Although the expected difference between $\sigma$ and $\mathrm{w}$ at $2.1 \mathrm{~K}$ is quite $\operatorname{smal1}\left(\left(\mathrm{T} / \mathrm{T}{ }_{\mathrm{c}}\right)^{2}=\right.$ 0.10 ), it is interesting to study the temperature dependence of the measured value of $\sigma$ in order to make a comparison with theory. More importantly, such a study is a test of the validity of EHD nucleation theory used to determine $\sigma$.

A graph of the surface tension $\sigma$ calculated from the threshold ratios $\mathrm{P}_{+} / \mathrm{P}_{-}$measured for sample 315 between $1.4 \mathrm{~K}$ and $2.1 \mathrm{~K}$ is shown in Fig. 5.17, for three different assumptions regarding the type of nucleation. As shown in Fig. 5.17 the curves for a11 three assumptions are consistent with the expected temperature dependence; the slopes yield $\delta_{\sigma} \approx 0$ for homogeneous nucleation and $\delta_{\sigma} \approx 0.1 \mathrm{~K}^{-2}$ and $\delta_{\sigma} \approx 0.2 \mathrm{~K}^{-2}$ for $\Delta \psi=50 \mathrm{~K}$ and $100 \mathrm{~K}$, respective1y, assuming inhomogeneous nucleation. Thus EHD nucleation theory provides an accurate description of the thresholds for drop formation and breakup below 2.1K. However, the systematic errors present in the theoretical analysis are too large to allow confidence in the measurement of $\delta_{\sigma}$, which depends upon smal1 changes in $\sigma$. In the following section we use the value ${ }^{5.15} \delta_{\sigma}=\mathrm{T}_{\mathrm{c}}{ }^{-2}$ in a careful analysis of the measured EHD-FE phase diagram to determine the surface energy $w$, which can be directly compared with theory. 


\section{Free-Exciton-Electron-Hole Drop Phase Diagram}

In this section we present a detailed measurement and analysis of the threshold phase diagram for $\mathrm{FE}$ and $\mathrm{EHD}$ in ultrapure $\mathrm{Ge}$ at temperatures between $1.2 \mathrm{~K}$ and $2.0 \mathrm{~K}$, which we believe is the most careful freqment of this problem to date. A brief account of this work has appeared

previously. ${ }^{5.16}$ We observe very large deviations from the simple phase diagram expected for ordinary liquids. These departures from simple behavior are caused by the impossibility of attaining thermodynamic equilibrium in the FE-EHD system, due to metastable effects of the EHD surface tension and the finite lifetimes of EHD and FE. We find excellent quantitative agreement between the measured threshold phase diagram and that calculated using EHD nucleation theory as described in Chapters 2 and 3. Because the calculated thresholds are sensitive functions of the EHD condensation energy $\phi$ and the surface tension $\sigma$, a fit of theory to experiment yields accurate values for these parameters; $\phi=1.9 \pm 0.2 \mathrm{meV}$ and $\sigma=(2.6 \pm 0.3) \times 10^{-4}$ erg $\mathrm{cm}^{-2}$ at $\mathrm{T}=0$. In ordinary usage, the term "phase diagram" assumes thermodynamic equilibrium between species and is thus not strictly applicable to the EHD-FE system. Because this system is characterized by metastability and other non-equilibrium processes at low temperatures $(T \lesssim 3 K$ ), the phase diagram for $\mathrm{EHD}$ and $\mathrm{FE}$ in the conventional sense cannot be directly measured and has little physical meaning. Hereafter we use the term EHD-FE phase diagram to denote a plot vs. temperature of the threshold FE densities $\mathrm{n}_{+}$and $\mathrm{n}_{-}$for the creation and destruction of 
EHD, which define the regions of stability for EHD in the $n, T$ plane. Although these thresholds are determined by non-equilibrium processes, the physical meaning of such a plot corresponds to that for ordinary liquids.

In the orginal experimental work $5 \cdot 1,5.8,5.17,5.18$ on the EHD-FE phase diagram, thermodynamic equilibrium was assumed in order to extract a value for the condensation energy $\phi$ from threshold data. These values, typically $\phi \approx 1.5 \mathrm{meV}$, differ substantially from more reliable spectroscopic measurements, $5.19,5.20$ typically $\phi \approx 1.9 \mathrm{meV}$. Recent threshold measurements,,$^{5.21,5.22}$ analyzed with the same assumption, yield similar values $\phi \approx 1.6 \mathrm{meV}$. Westerve1t, Staehli, and Haller ${ }^{5.23}$ first correctly explained that the origins of this discrepancy were non-equilibrium effects in the FE-EHD system as described in Chapters 2 and 3. The same theoretical conclusion was reached independently by Silver. 5.24 Westervelt et a1. $5.9,5.23$ also reported the first measurements of the complete FE-EHD phase diagram for Ge including the thresholds for the destruction of EHD, and found good qualitative agreement between experiment and EHD nucleation theory, including the disappearance of hysteresis below $\mathrm{T} \approx 1.4 \mathrm{~K}$. The ratio $\mathrm{P}_{+} / \mathrm{P}_{-}$of the excitation thresholds was used in this work $5.9,5.23$ to determine the EHD surface energy $w=(2.4$ to 2.8$) \times 10^{-4} \mathrm{erg} \mathrm{cm}^{-2}$ as described in Section 53. Subsequent1y, Etienne et a1..$^{5.25}$ have also measured the FE-EHD phase diagram including hysteresis with similar experimental results. However, they do not find good agreement between threshold data and their treatment of EHD nucleation, and conclude only that $w$ lies in the range 
(1.8 to 2.6$) \times 10^{-4} \mathrm{erg} \mathrm{cm}^{-2}$; we discuss their work further below. Recently, Thomas et al. 5.20,5.13 claimed to have accurately measured the FE-EHD phase diagram spectroscopically, simply by determining the condensation energy $\phi$ and the density of states for FE which are then substituted into the theoretical expression for the saturated FE density $\mathrm{n}_{\mathrm{s}}$ (see Eq. 2.8). From the discussion above it is clear that hysteresis and lifetime effects dominate the phase diagram below $3 \mathrm{~K}$, and that the saturated density $\mathrm{n}_{\mathrm{S}}$ is therefore inapplicable. Furthermore, the absolute accuracy of their calculated phase diagram is only a factor $\approx 3$ at $2 \mathrm{~K}$, no better than that obtained by calibrated threshold measurements.

Two central difficulties must be overcome in order to produce a careful measurement of the FE-EHD phase diagram which can be quantitatively compared with nucleation theory. First, it is imperative that the existence of hysteresis effects be correctly accounted for. Thus both branches of the phase diagram, $n_{+}$and $n_{-}$, must be measured using a stable, noise-free source of excitation cycled in a well-defined and reproducible manner, as described in Chapter 4. Because the observed thresholds vary with the time of observation $\Delta t$ at low temperatures, (see Fig. 5.13), the data should also be recorded at a fixed value of this time $\Delta t$ which can later be used in the analysis. Second, a technique must be developed to quantitatively relate the observed thresholds in excitation power to the peak value of the $\mathrm{FE}$ density $\mathrm{n}$ within the Ge crystal. In the discussion below, we give a detailed analysis of this problem and describe the procedure used in this experiment. 
The largest value of the FE density within a sample of Ge uniformly excited across one entire surface (the geometry used in this work) is given by

$$
n=P \tau_{x} n / h \nu L_{\text {eff }} \text {, }
$$

where $P\left(\mathrm{~mW} \mathrm{~cm}^{-2}\right)$ is the absorbed excitation power for photons of energy hv, $\tau_{x}$ is the FE lifetime, $\eta$ is the quantum efficiency for the production of excitons, and $\mathrm{L}_{\text {eff }}$ is the effective depth of penetration of FE into the sample. For surface excitation $L_{\text {eff }}$ is simply equal to the $\mathrm{FE}$ diffusion length $\mathrm{L}=\left(\mathrm{D} \tau_{\mathrm{x}}\right)^{1 / 2}$ with $\mathrm{D}$ the diffusion constant; for volume excitation assuming negligible surface recombination. $\mathrm{L}_{\text {eff }}=\mathrm{L}+\ell$ where $\ell \approx 1 \mathrm{~mm}$ is the absorption 1ength of $1.52 \mu \mathrm{m}$ radiation. Another measure of the peak FE density $\mathrm{n}$ is the intensity $I_{x}$ of the FE Iuminescence below the threshold for drop formation; for the experimental geometry used here we have

$$
I_{x}=C n L_{\text {eff }}=\operatorname{CP} \tau_{x} n
$$

where $\mathrm{C}$ is a constant of proportionality. Note that $\mathrm{I}_{\mathrm{x}}$ from $\mathrm{Eq} \cdot(5.11)$ for a given value of the excitation $P$, is independent of the depth of penetration $\mathrm{L}_{\text {eff }}$ of $\mathrm{FE}$ into the sample. For the apparatus described in Chapter 4, the excitation power $\mathrm{P}$ is accurately monitored and calibrated, and the photon energy $h \nu$ is well known. Thus the primary uncertainties in Eqs. (5.10) and (5.11) are due to the quantum efficiency, the FE lifetime and the spatial distribution of $\mathrm{FE}$ within the sample. For quantitative comparison of the measured phase diagram with theory it is essential that these parameters do not vary with temperature; 
their absolute values are somewhat less important.

We can test the effects of the quantum efficiency $\eta$, the FE Iifetime $\tau_{x}$, and the spatial distribution of $\mathrm{FE}$ by comparing the hysteresis curves obtained for EHD and $\mathrm{FE}$ using volume and surface excitation on the same sample at the same temperature. We also test the long-term reproducibility of the data for these two cases by repeating the measurements. Hysteresis curves for $\mathrm{EHD}$ in sample 315 at $\mathrm{T}=1.82 \mathrm{~K}$ are shown in Fig. 5.18 for surface excitation and in Fig. 5.19 for volume excitation. In both figures the data were recorded in order of decreasing excitation, first for the upgoing curve, then for the downgoing curve. After the data for Run 1 were recorded, (elapsed time $\sim 4 \times 10^{3} \mathrm{sec}$ ), the process was repeated to obtain Run 2 as indicated in Figs. 5.18 and 5.19. As shown in Fig. 5.18, the data obtained using surface excitation do not reproduce well for Runs 1 and 2 , despite the fact that the short-term reproducibility from point to point is very good. Both the EHD signals $\mathrm{I}_{\mathrm{d}}^{+}$and $\mathrm{I}_{\mathrm{d}}{ }^{-}$, and the excitation thresholds $\mathrm{P}_{+}$and $\mathrm{P}_{-}$differ substantially for the two runs shown in Fig. 5.18. On the other hand, the data obtained using volume excitation, shown in Fig. 5.19, are strictly reproducible. This evidence indicates that the sample surface can have a considerable effect on the density of FE produced by optical excitation. Direct evidence that the sample surface can rapidly destroy $\mathrm{FE}$ in Ge was recently presented by Elliot et al. 5.26 It is not clear whether the difference between Runs 1 and 2 in Fig. 5.18 is caused by the relatively large initial excitation $\mathrm{P}_{\max }=14.0 \mathrm{~mW} \mathrm{~cm}-2$ used to record 
the downgoing points, or by other effects. However, volume excitation is obviously preferable for quantitative measurements of the EHD-FE phase diagram. Previously reported measurements of the EHD-FE phase diagram $5.9,5.25$ were taken using surface excitation and are thus subject to irreproducible effects. Because the threshold ratios $\mathrm{P}_{+} / \mathrm{P}_{-} \approx 4.8$ and $\mathrm{P}_{+} / \mathrm{P}_{-} \approx 5.4$ for Runs 1 and $2 \mathrm{in} \mathrm{Fig} .5 .18$ reproduce reasonably wel1, the effect of surface excitation upon the measurements given in Section 53 is expected to be less serious.

By comparing the FE signals $\mathrm{I}_{\mathrm{x}}^{+}$for surface and volume excitation recorded below the threshold $\mathrm{P}_{+}$, where $\mathrm{I}_{\mathrm{X}}{ }^{+} \propto \mathrm{P}$ approximately, we obtain a measure of the quantum efficiency $n$. For sample 315 at $\mathrm{T}=1.80 \mathrm{~K}$, we find that $\mathrm{I}_{\mathrm{x}}$, and therefore the product $\eta_{\mathrm{x}}$ from Eq. 5.11, are $\approx 3.6$ times smaller for surface excitation, using the same values of the absorbed photon $f l u x ~ P / h \nu$ in both cases. Even if the surface recombination rate for volume excitation is very large, the overall quantum efficiency $\eta$ is no smaller than $\eta=[1+(L / \ell)]^{-1} \approx 60 \%$ for $\mathrm{L} \approx 0.7 \mathrm{~mm}$ and $\ell \approx 1.0 \mathrm{~mm}$, the values appropriate for this sample. Assuming that the lifetime $\tau_{x}$ is the same for both cases, we can thus conclude that $\eta \approx 16 \%$ for surface excitation on this sample. This value of $\eta$ corresponds to the surface recombination velocity $\mathrm{S}=\left(\mathrm{L} / \tau_{\mathrm{x}}\right)\left(\eta^{-1}-1\right) \approx 1 \times 10^{5} \mathrm{~cm} \mathrm{sec}{ }^{-1}$, considerably larger than the estimate $S \approx 10^{2} \mathrm{~cm} \mathrm{sec}^{-1}$ for free carriers at room temperature (see Section 42). Thus the surface recombination of $\mathrm{FE}$ can be an important factor in the FE density calibration of threshold measurements. Note that the threshold $\mathrm{P}_{+}$in Fig. 5.18 for surface excitation (corrected 
for the difference in mean photon energy $h \nu)$ is $\approx 1.5$ times greater than that for volume excitation. Using the appropriate values for $\mathrm{L}_{\text {eff }}$ in Eq. (5.11), we find the ratio 3.6 for the product $\eta \tau_{\mathrm{x}}$ for surface and volume excitation, in excellent agreement with the result from $I_{x}$ above.

Because the mechanism which determines the short FE 1ifetime $\tau_{x}$ in the samples studied has not yet been positively identified, it is important to establish that this parameter is in fact a true exponential lifetime, and to study the variation of $\tau_{x}$ with temperature and excitation leve1. As described in Chapter 4, we measured the 1ifetime $\tau_{x}$ for several samples by recording the decay of the FE 1uminescence at 4.2K following short, pulsed GaAs laser excitation, after the EHD luminescence had disappeared. In all cases (see Table 4.2) this decay was exponential as expected, indicating that $\tau_{x}$ does not vary with $F E$ density $n$. Hensel et a1. ${ }^{5.13}$ have suggested that $\tau_{x}$ is determined by recombination at impurity atoms and therefore that $\tau_{x} \propto N_{i}^{-1}$, where $\mathrm{N}_{i}$ is the total impurity concentration. Inspection of Table 4.2 shows no correlation between $\tau_{x}$ and $N_{i}$, ruling out this mechanism. However, the shorter values of $\tau_{x}$ in Table 4.2 are associated with the more highly dislocated samples, suggesting dislocations as an active recombination site.

Unfortunately, the lifetime $\tau_{x}$ could not be directly measured at temperatures much less than $4 \mathrm{~K}$, because the EHD luminescence cut-off time becomes very long. 5.4 In order to study the variation of $\tau_{x}$ with temperature and excitation power, the steady-state $\mathrm{FE}$ luminescence 
signal $\mathrm{I}_{\mathrm{X}}^{+}$was recorded vs. $\mathrm{P}$ using volume excitation at levels below the threshold $P_{+}$for drop formation at various temperatures between $1.5 \mathrm{~K}$ and $4.2 \mathrm{~K}$. The results are shown vs. temperature as a series of constant-excitation contours in Fig. 5.20; the excitation power $\mathrm{P}$ for each curve is indicated. As shown in Fig. 5.20, the FE signal $I_{x}$ for a given excitation is accurately independent of temperature, except for the signals recorded at $4.2 \mathrm{~K}$, which are slightly smaller. From Eq. (5.11) for $I_{x}$, we conclude that the product $\eta \tau_{x}$ is practically temperature independent between $1.5 \mathrm{~K}$ and $4.2 \mathrm{~K}$. Assuming that the overall quantum efficiency $\eta$ for volume excitation does not vary with $\mathrm{T}$ (which is true both in the 1imt ts of negligible and strong surface recombination), the data of Fig. 5.20 indicate that the FE lifetime is constant in this temperature range.

This study of the $F E$ signal $I_{x}$ vs. excitation is important, because the threshold $\mathrm{FE}$ densities $n_{+}$and $n_{-}$at low temperatures are comparable to the concentration of impurities and other defects in the samples studied (see Section 42), and FE trapping by these defects could be significant in principle. A plot of $\mathrm{I}_{\mathrm{x}}^{+}$vs. $\mathrm{P}$ for volume excitation of sample 315 below the threshold $\mathrm{P}_{+}$for EHD formation at $\mathrm{T}=2.00 \mathrm{~K}$ is given in Fig. 5.21. As shown in this figure, the FE signal $I_{x}$ varies linearly with excitation for $\mathrm{P} \gtrsim 0.5 \mathrm{~mW} \mathrm{~cm}^{-2}$ as indicated by the solid line. However, for smaller values of excitation $\mathrm{P} \lesssim 0.5 \mathrm{~mW} \mathrm{~cm}^{-2}$, the slope of $\mathrm{I}_{\mathrm{X}}$ vs. $\mathrm{P}$ decreases, suggesting the existence of a small concentration of active traps for FE. At 4.20K similar behavior is observed, except the break in slope of $I_{X}$ vs. $P$ 
occurs at a slightly larger value of the excitation, $\mathrm{P} \approx 0.8 \mathrm{~mW} \mathrm{~cm}^{-2}$. Thus the FE density produced by volume excitation is somewhat smaller than that predicted assuming $n \propto P$. Because the excitation thresholds $\mathrm{P}_{+}$and $\mathrm{P}_{-}$at $\mathrm{T} \sim 1.5 \mathrm{~K}$ are of the order $\sim 0.3 \mathrm{~mW} \mathrm{~cm}^{-2}$ in this sample (see Fig. 5.8), non-linearity in $I_{x}$ vs. P such as that shown in Fig. 5.21 can be significant, and should be included in the FE density calibration.

Finally, we consider the possibility that the FE diffusion length $L=\left(D \tau_{x}\right)^{1 / 2}$ changes with temperature. This parameter enters the $\mathrm{FE}$ density calibration through the effective depth $\mathrm{L}_{\text {eff }}$ of $\mathrm{FE}$ in the sample, as indicated for Eqs. (5.10) and (5.11) above. To our knowledge, the only measurement of the FE diffusion constant $D$ at low temperatures $(\mathrm{T}<4 \mathrm{~K})$ in $\mathrm{Ge}$ is that of Pokrovskii and Svistunova ${ }^{5.27}$ who find $D \approx 1500 \mathrm{~cm}^{2} \mathrm{sec}^{-1}$ at $3 \mathrm{~K}$ below the threshold FE density for drop formation. This valve of $\mathrm{D}$ is consistent with that estimated ${ }^{5.13}$ from the carrier-phonon scattering time $\tau_{\mathrm{p}} \approx 10^{-9} \mathrm{sec}$ in Ge at $3 \mathrm{~K}^{5.28}$, $\mathrm{D}=\tau_{\mathrm{p}} \mathrm{kT} / \mathrm{m}^{*}=1400 \mathrm{~cm}^{2} \mathrm{sec}^{-1}$. Therefore we assume that $\mathrm{FE}$ diffusion at low temperatures and $\mathrm{FE}$ density is limited by phonon-scattering. Because the value of the scattering time $\tau_{\mathrm{p}}$ is proportional to $\mathrm{T}^{-3 / 2}$ for free carriers, ${ }^{5.28}$ we expect that the FE diffusion constant varies as $\mathrm{D} \propto \mathrm{T}^{-1 / 2}$ with temperature. Thus the $\mathrm{FE}$ diffusion length $L=\left(D \tau_{x}\right)^{1 / 2} \propto T^{-1 / 4}$ is expected to be only slowly temperature- . dependent; as $\mathrm{T}$ increases from $1.3 \mathrm{~K}$ to $2.0 \mathrm{~K}$, the range of our threshold measurements, L decreases by only $\approx 20 \%$. For surface excitation a corresponding change in expected in $\mathrm{L}_{\text {eff }}=\mathrm{L}$ in Eqs. (5.10) and (5.11). For volume excitation, the temperature variation of $\mathrm{L}_{\text {eff }}=\mathrm{L}+\ell$ is 
even smaller, because the absorption length $\ell \approx 1 \mathrm{~mm}$ is larger than $\mathrm{L} \approx 0.7 \mathrm{~mm}$, and this variation can be safely neglected.

In the following paragraph we describe the method actually used to measure the FE-EHD phase diagram for Ge. Volume excitation was used in recording all of the data in order to avoid irreproducible effects of the sample surface such as those described above (see Figs. 5.18 and 5.19) and to simplify the analysis of the spatial distribution of free excitons. Hysteresis curves for both the EHD and FE signals $\mathrm{I}_{\mathrm{d}}^{ \pm}$and $\mathrm{I}_{\mathrm{x}}^{ \pm}$such as those shown in Fig. 5.2 were recorded during one experimental run at a number of temperatures between $1.2 \mathrm{~K}$ and $2.0 \mathrm{~K}$. At higher temperatures the maximum excitation available with this apparatus was insufficient to reach the threshold $\mathrm{P}_{+}$for drop formation. Excitation thresholds $\mathrm{P}_{+}$and $\mathrm{P}_{\text {- }}$ were extrapolated from plots of $\mathrm{I}_{\mathrm{d}}{ }^{1 / 2}$ vs. $\mathrm{P}$ for the data at each temperature as described in section 51 . In order to correct for the non-1inearity of $\mathrm{I}_{\mathrm{x}}$ vs. $\mathrm{P}$ (see Fig. 5.21), we assumed that the thresholds $n_{+}$and $n_{-}$in FE density were proportional to the $\mathrm{FE}$ signals $\mathrm{I}_{\mathrm{x}}{ }^{+}$and $\mathrm{I}_{\mathrm{x}}{ }^{-}$measured for each temperature at the corresponding excitation thresholds $\mathrm{P}_{+}$and $\mathrm{P}_{-}$, as shown in $\mathrm{Eq}$. (5.11). Because the length $\mathrm{L}_{\text {eff }}$ in Eq. (5.11) is expected to be essentially temperature-independent for volume excitation, this method yields accurate relative values for $n_{+}$and $n_{-}$. To determine the constant of proportionality $\mathrm{C}$ in $\mathrm{Eq} \cdot(5.11)$ and thus the absolute FE density, we used Eq. (5.10) with $\eta=1$ for the FE density vs. P and used Eq. (5.11) to compare this value with the $\mathrm{FE}$ signal $I_{\mathrm{x}}$ recorded at $2.0 \mathrm{~K}$ for the relatively large excitation $P \approx 4 \mathrm{~mW} \mathrm{~cm}^{-2}$. The values of the parameters 


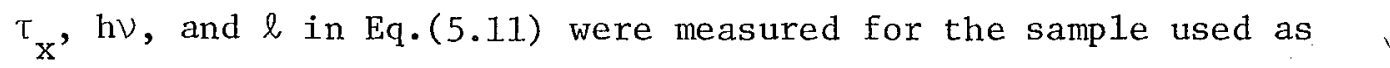
described in Chapter 4, and the FE diffusion constant was assumed to have the temperature-independent value ${ }^{5.27} \mathrm{D}=1500 \mathrm{~cm}^{2} \mathrm{sec}^{-1}$. Compared to the values obtained by simply assuming $\mathrm{n} \propto \mathrm{P}$, the $\mathrm{FE}$ thresholds determined in this manner exhibited a steeper temperature dependence, and were smaller by a factor $\approx 1.5$ at $\mathrm{T} \sim 1.3 \mathrm{~K}$. Thus, the relative values of the threshold $\mathrm{FE}$ densities $\mathrm{n}_{+}$and $\mathrm{n}_{-}$obtained by this method are substantially more accurate than those obtained from excitation thresholds alone as in earlier work; ${ }^{5.9,5.25}$ no absolutely calibrated measurements of both $\mathrm{n}_{+}$and $\mathrm{n}_{-}$have been previously reported.

The measured FE-EHD phase diagram for sample 315 obtained in this manner is shown in Fig. 5.22 as the data points together with a theoretical fit described below. The qualitative features of Fig. 5.22 are in excellent agreement with EHD nucleation theory as described in Chapters 2 and 3 and with the hysteresis data presented in Section 51. The measured FE density thresholds $\mathrm{n}_{+}$and $\mathrm{n}_{-}$shown in Fig. 5.22 display a pronounced hysteresis which decreases with temperature and disappears below $\mathrm{T} \approx 1.4 \mathrm{~K}$. Both thresholds vary only slowly with temperature and lie we11 above the saturated $\mathrm{FE}$ density $\mathrm{n}_{\mathrm{s}}$ predicted by equilibrium thermodynamics, indicated by the dashed curve. Furthermore, the slope of the upgoing branch $n_{+}$of the phase diagram is substantially smaller than that of $\mathrm{n}_{\mathrm{s}}$, explaining the small values obtained for previous "threshold" measurements $5 \cdot 1,5.8 \cdot 5 \cdot 17,5.18,5.21,5.22$ of the condensation energy $\phi$. From the data of Fig. 5.22 it is clear that the FE-EHD phase 
diagram below $2 \mathrm{~K}$ is dominated by the effects of metastability and the EHD lifetime, and that equilibrium thermodynamics provides a very poor description of the system. Any threshold experiment which neglects these non-equilibrium effects in either the data taking procedure or the analysis can be expected to yield inaccurate results.

In order to make a quantitative comparison of the measured FE-EHD phase diagram with nucleation theory and accurately measure the EHD surface energy $w$ and the condensation energy $\phi$, a least-squares fit of the thresholds $n_{+}$and $n_{-}$predicted by the theory described in Chapters 2 and 3 to the data of Fig. 5.22 was made using $w$ and $\phi$ at $\mathrm{T}=0$ as adjustable parameters. Because the calculated phase diagram is very sensitive to the values of $w$ and $\phi$, they must be adjusted to make a valid comparison. For the same reason, this precedure is an accurate and reliable method to determine the values of these parameters. In order to obtain the best fit at the lowest temperatures, the coefficient $c$ of the temperature-independent evaporation rate 5.29 of EHD was also included in the theory, as a third adjustable parameter.

To ensure accurate results the temperature dependence of the parameters $\sigma, \phi$, and $\mathrm{n}_{\mathrm{o}}$ and the actual non-parabolic density of states for $F E$ were included in the theory. As discussed in Section 53, the surface energy $\sigma$ is expected to vary with temperature as $\sigma=w\left(1-\delta_{\sigma} T^{2}\right)$; we used the value $\delta_{\sigma}=\mathrm{T}_{\mathrm{c}}{ }^{-2}$ with the critical temperature ${ }^{5.14} \mathrm{~T}_{\mathrm{c}}=6.5 \mathrm{~K}$. For the condensation energy we used $\phi=\phi_{0}\left(1+\delta_{\phi} \mathrm{T}^{2}\right)$, where the coefficient $\delta_{\phi}=0.18 \mathrm{~K}^{-2}$ has been measured spectroscopically $\cdot^{5.20}$ Recently, the actual non-parabolic density of states for FE in Ge has 
been measured by Thomas et al.,$^{5.20}$ and accurately calculated by Altare11i and Lipari ${ }^{5.30}$ with exce1lent agreement between theory and experiment. Because the experimental results are significantly broadened at the low energy edge by the spectral resolution of their apparatus, we could not use them to calculate the actual effective density of states $D_{x}$ for $F E$ which enters $E q .(2.8)$ for the saturated $F E$ density $\mathrm{n}_{\mathrm{s}}$. Instead, we calculated $\mathrm{D}_{\mathrm{x}}$ by numerically integrating the dispersion relation given for FE by Altarelli and Lipari. ${ }^{5.30}$ The results for $1 \mathrm{~K}<\mathrm{T}<5 \mathrm{~K}$ accurately fit the polynomial expansion (error $<0.5 \%$ ):

$$
D_{x}=\gamma_{x}\left(m * k T / 2 \pi \hbar^{2}\right)^{3 / 2}\left(0.98+0.045 T+0.0106 T^{2}\right)
$$

where $m^{*}=0.436 m_{0}$ is the density-of-states effective mass and $T$ is expressed in K. An at tempt to include the effect of non-parabolicity on the FE mean speed $\bar{v}$ was also made by assuming spherical symmetry and numerically evaluating the integral for $\bar{v}$ with the result

$$
\overline{\mathrm{v}}=\left(8 \mathrm{kT} / \pi \mathrm{m}^{*}\right)^{1 / 2}\left(1.14-0.050 \mathrm{~T}+0.0020 \mathrm{~T}^{2}\right)
$$

where $\mathrm{m}^{*}=0.436 \mathrm{~m}_{\mathrm{O}}$ and $\mathrm{T}$ is expressed in $\mathrm{K}$ as above. From Eqs. (5.12) and (5.13) we find that the temperature corrections to $D_{x}$ and $\bar{v}$ due to non-parabolicity are negligible below $2 \mathrm{~K}$; even at $4 \mathrm{~K}$ these corrections are relatively small, $\lesssim 35 \%$. The most significant result of this calculation is the "improved" value $\mathrm{m}^{*}=0.436 \mathrm{~m}_{\mathrm{o}}$ for the $\mathrm{FE}$ density-ofstates effective mass, which is $\approx 30 \%$ larger than the value $\mathrm{m}^{*}=0.335 \mathrm{~m}_{0}$ from Table 3.1, which was also used by Thomas et a1. ${ }^{5.20}$ to estimate the size of the effect of non-parabolicity on $\mathrm{D}_{\mathrm{x}}$. Thus we conclude 
that the effect of recent measurements ${ }^{5.20}$ and calculations ${ }^{5.30}$ of the FE density of states on $\mathrm{D}_{\mathrm{x}}$ below $4 \mathrm{~K}$ is primarily accounted for by a simple change in the value of $\mathrm{m}^{*}$. Nevertheless, the full expressions Eqs. (5.12) and (5.13) were used in the analysis below. The values of the other parameters used to calculate the phase diagram are those given in Table 3.1, with the exception of the maximum generation rate $G_{M}$, the observation time $\Delta t$, the lifetimes $\tau_{0}$ and $\tau_{x}$, and the impurity concentration $N_{i}$; for these quantities the actual experimental values were used (see Chapter 4).

The fitting procedure was carried out for two separate assumptions: homogeneous nucleation of EHD and inhomogeneous nucleation on neutral impurity atoms with the value $\Delta \psi=50 \mathrm{~K}$ for the barrier lowering (see Chapter 2). The FE density thresholds $n_{+}$and $n_{-}$were numerically calculated from the threshold condition Eq. (3.15); the threshold density $\mathrm{n}_{\mathrm{e}}$ for a significant population of EHD in dynamic equilibrium with the FE gas was also found from Eq. (3.18). Whenever the threshold $\mathrm{n}_{\mathrm{e}}$ was respectively greater or smaller than the calculated values of $\mathrm{n}_{+}$or $\mathrm{n}_{-}$, this threshold was used to compare with the data; this algorithm corresponds to the physical situation as discussed in Chapters 2 and 3 . Because the relative accuracy of the FE density calibration for $n_{+}$and $n_{-}$described above is expected to be much better (by a factor $>10$ ) than the absolute accuracy, the absolute calibration was not used in the fitting procedure. Thus the shape of the measured phase diagram was accurately fitted; the measured absolute values for $\mathrm{n}_{+}$and $\mathrm{n}_{-}$were used as a check on the results. The actual calculations 
were done numerically on the CDC 7600 computer at Lawrence Berkeley Laboratory. A rough estimate of the error in the resulting values of the fitted parameters was made by visually comparing the thresholds calculated for slightly different values of $w, \phi_{0}$ and $c$ with the experimental data.

The threoretical FE-EHD phase diagram obtained by this procedure is shown as the solid curves in Fig. 5.22. The results obtained assuming homogeneous and inhomogeneous nucleation differed very little; the fit obtained assuming EHD formation on impurity atoms is slightly better, as we expect from the discussion in Section 53, and is given in Fig. 5.22. As shown in Fig. 5.22, we find excellent agreement between the experimental data and the calculated curves over the entire temperature range of the measurement. This agreement is a strong confirmation of the validity of EHD nucleation theory, and provides confidence in the results obtained for the fitted parameters $w$ and $\phi_{0} \cdot$ Recently, the threshold FE density $\mathfrak{n}_{+}$at low temperatures $(T \gtrsim 2 K)$ has also been measured for $\mathrm{Si}$ by Dite et a1. 5.31 These measurements agree reasonably well with nucleation theory as shown in Fig. 3.5, providing additional evidence for the validity of this approach. The lack of agreement between theory and experiment found by Etienne et a1. 5.25 is due to their use of surface excitation, which can yield irreproducible results (see Figs. 5.18 and 5.19) their unjustified assumption that $\mathrm{n} \propto \mathrm{P}$ for a11 temperatures (see Fig. 5.21), and the failure to include the term c in the analysis. 
The FE density scale shown in Fig. 5.22 is that from the theoretical calculation. The absolute calibration for the data points in Fig. 5.22 yields values of the FE density approximately four times smaller than the theory. To check on this discrepancy, the fitting procedure was repeated using absolute rather than the relative values for the data. The fit thus obtained was considerably poorer than that shown in Fig. 5.22, both qualitatively and quantitatively. Because the absolute accuracy of the FE density calibration is not expected to be better than roughly a factor of two, we feel that this discrepancy is not serious and therefore that the results shown in Fig. 5.22 are more accurate and reliable. However, it is surprising that the measured values of $n_{ \pm}$are smaller than the theory. Such an effect could be due to an increased FE lifetime in the interior of the sample, or possibly to a localized increase in the $\mathrm{FE}$ density in the strain well about dislocations, present in this sample.

Now we present the results for the EHD surface energy $w$, the condensation energy $\phi_{0}$ at $\mathrm{T}=0$, and the coefficient $c$, obtained by fitting the entire FE-EHD phase diagram as described above. Because the calculated phase diagram is quite sensitive to the values of $w$ and $\phi_{0}$, the fit accurately determines these important parameters. This measurement is also the first in which $\phi_{0}$ and w are both independently determined by threshold data. As mentioned previously, the results obtained assuming homogeneous and inhomogeneous nucleation differed only slightly. Overa11 we find 


\section{$00+04804840$}

$-135-$

$$
\begin{aligned}
& \phi_{0}=1.9 \pm 0.2 \mathrm{meV}, \\
& \mathrm{w}=(2.6 \pm 0.3) \times 10^{-4} \mathrm{erg} \mathrm{cm}-2 .
\end{aligned}
$$

The fit was sensitive only to the order of magnitude of $c$; for this parameter we find $c \sim 10^{5} \mathrm{sec}^{-1}$. Hensel et a1 $1^{5.13,5.17}$ have suggested that the temperature-independent evaporation rate of EHD arises from excitations in the e-h liquid near the surface caused by the radiative recombination of e-h pairs. The value $\mathrm{c} \sim 10^{5} \mathrm{sec}^{-1}$ above corresponds to the emission of one $\mathrm{FE}$ for each recombination within $\approx 15 \mathrm{~nm}$ of the EHD surface; this length is comparable to the surface thickness $\approx 20 \mathrm{~nm}$ for EHD lending credability to this model.

The value of the condensation energy $\phi_{0}=1.9 \pm 0.2 \mathrm{meV}$ from Eq. (5.14) obtained by fitting the phase diagram is in excellent agreement with the most careful spectroscopic measurement 5.6 $\phi=1.8 \pm 0.2 \mathrm{meV}$, and with the theoretical value $\phi_{\mathrm{o}}=1.8 \mathrm{meV}$ obtained from the most detailed calculations of the e-h liquid pair binding energy ${ }^{5.32} \mathrm{E}_{\mathrm{g}}=5.9 \mathrm{meV}$ and the $\mathrm{FE}$ binding energy 5.30 $E_{x}=4.15 \mathrm{meV}$. Thus we have conclusively shown that the long-standing discrepancy between so-called "threshold" and spectroscopic measurements of $\phi_{0}$ is completely explained by the proper application of EHD nucleation theory. Although the fact that this discrepancy can be resolved by nucleation theory has been discussed previous 1 y, 5.9,5.23-5.25 this is the first actual measurement of the condensation energy from threshold data which includes metastability and lifetime effects in the analysis. In fact, the accuracy of this method is comparable to 
that obtained spectroscopically. The sensitivity of the measured thresholds to the value of $\phi$ is due to the fact that the saturated FE density $\mathrm{n}_{\mathrm{S}} \propto \exp (-\phi / \mathrm{kT})$ is a very sensitive function of $\phi$ at temperatures $\sim 2 \mathrm{~K}$. For $\mathrm{T} \gtrsim 2 \mathrm{~K}$ we can measure $\phi$ from the absolute value of the calibrated threshold FE density $n_{+}$by simply scaling the results of nucleation theory to fit the data, assuming $\mathrm{n}_{+} \propto \mathrm{n}_{\mathrm{s}}$, which is true for classical nucleation. In this way we find $\phi \approx 2.1 \mathrm{meV}$ from the data at 2.0K in Fig. 5.22, which agrees reasonably well with the more accurate value given above.

A list of experimentally measured values of the EHD surface tension $\sigma$ and the theoretically calculated surface energy $\mathrm{w}(\sigma$ at $\mathrm{T}=0)$ reported to date is given in Table 5.2. As shown in Table 5.2, the original calculations ${ }^{5.33-5.36}$ for the surface energy yielded values typically $\mathrm{w} \approx 1 \times 10^{-4} \mathrm{erg} \mathrm{cm}^{-2}$. Recently, by including exchange and and correlation effects in the gradient expansion used in more complete calculations, Vashishta et al. 5.37 and Rose et a1. ${ }^{5.38}$ have obtained considerab1y larger results $w=(3.5$ to 4$) \times 10^{-4} \mathrm{erg} \mathrm{cm}^{-2}$ and $w=(2.4 \pm 0.5) \times 10^{-4} \mathrm{erg} \mathrm{cm}^{-2}$, which are more reliable. The first measurements of the EHD surface tension $\sigma$ were those of Bagaev et al. ${ }^{5.39}$ and Westervelt et a1. $5.23,5.9$ Bagaev et a1. estimated $\sigma$ from the temperature dependence of the drop concentration $N$ measured by light scattering and absorption. Making the ad hoc assumption that $\mathrm{N} \propto \mathrm{J}_{+}$, the initial EHD formation rate for chopped excitation, they found $\sigma \approx 1.8 \times 10^{-4} \mathrm{erg} \mathrm{cm}^{-2}$. Because the rate $\mathrm{J}_{+}$ is expected to rapidly decrease in time following the leading edge of an exciting pulse (see Sections 31 and 32, and Ref. 5.10), the 
accuracy of this assumption must be questioned. As shown in Table 5.2, Westervelt et a1. $5.9,5.23$ found $w \approx 2.6 \times 10^{-4} \mathrm{erg} \mathrm{cm}^{-2}$ from threshold data, in agreement with the value given above in Eq. (5.13) using the method described in Section 53. This method has the disadvantage that the temperature-dependence of the individual thresholds $\mathrm{P}_{+}$and $\mathrm{P}_{-}$is not included in the analysis. Two measurements of $\sigma$ by Etienne et al. using different methods are also shown in Table 5.2. A third experiment by this group 5.41 measured the shift in the EHD 1uminescence 1ine due to the effect of the surface tension on small drops, but did not independently determine $\sigma$. The first value ${ }^{5.25} \sigma=(1.8$ to 2.6$) \times$ $10^{-4} \mathrm{erg} \mathrm{cm}^{-2}$ was obtained from their measurement of the FE-EHD phase diagram. Because these authors were unable to find good agreement with nucleation theory as discussed above, they measured $\sigma$ by adjusting this parameter so that the slope vs. $1 / T$ of the calculated thresholds $\mathrm{n}_{+}$between $2 \mathrm{~K}$ and $4.2 \mathrm{~K}$ yielded an apparent value of the condensation energy $\phi=1.47$ to $1.55 \mathrm{meV}$, assuming the true value is given by $2.0 \mathrm{meV}$. The second technique used by this group was the observation of capillary-wave resonances of EHD in the attenuation of ultrasonic waves at $1.8 \mathrm{~K}$. In principle this method provides a direct measurement of the surface tension. However, the value deduced for $\sigma$ from the observed resonant frequency is proportional to the cube of the drop radius $\mathrm{R}$. Because the value $\mathrm{R}=2 \mu \mathrm{m}$ for the initial drop radius used in the analysis was not measured in this experiment, and is not an intrinsic property of the system $(\mathrm{R} \approx 8 \mu \mathrm{m}$ at $2 \mathrm{~K}$ from Ref. 5.8) it could easily be in error by at least $50 \%$. Thus their result $\sigma=3 \times 10^{-4} \mathrm{erg} \mathrm{cm}^{-2}$ is 
uncertain by a factor $\gtrsim 3$. Until $\mathrm{R}$ is accurately measured with the ultrasonic attenuation on the same apparatus, this method cannot be relied upon to yield accurate results.

Thus we believe that the measurement of the EHD surface energy $w=(2.6 \pm 0.3) \times 10^{-4}$ erg $\mathrm{cm}^{-2}$ from Eq. (5.15) obtained by fitting the entire FE-EHD phase diagram is substantially more accurate and reliable than those previously reported. As shown in Table 5.2, this value is in reasonable agreement with the recent calculations of Vashishta et a1..$^{5.37}$ and of Rose et al. ${ }^{5.38}$ This result also justifies a posteriori the measurement of $\sigma$ from threshold ratios $\mathrm{P}_{+} / \mathrm{P}_{-}$described in Section 53 and previously reported. $5.9,5.23$ 
CHAPTER 6. CONCLUSIONS

Because a detailed discussion of the application of nucleation theory to hysteresis and threshold data is given in Chapter 5, we briefly summarize here only the most important results. Using a modified form of the Becker and Döring rate equations, we calculated in Chapter 2 the non-equilibrium rates $J_{+}$and $J_{-}$of formation and breakup of electron-hole drops in a supersaturated gas of free excitons. These rates, largely determined by the value of the EHD surface energy w, yield sharp FE density thresholds $n_{+}$and $n_{-}$for EHD formation and breakup, and predict large metastable effects in the FE-EHD system above $\mathrm{T} \approx 1.4 \mathrm{~K}$. At temperatures $\lesssim 1.4 \mathrm{~K}$, the effect of the EHD lifetime becomes dominant, metastability is destroyed, and the thresholds are determined by the calculated population of EHD in dynamic equilibrium with the $\mathrm{FE}$ gas. In both cases, the calculated thresholds 1ie well above the saturated FE density $\mathbf{n}_{S}$, which determines the phase boundary for ordinary liquids.

In Chapter 3 we used these results to give a detailed quantitative theoretical description of the process of EHD nucleation from a FE gas in Ge. We calculated both the excitation hysteresis curves and the metastable time development expected for the EHD luminescence intensity. The calculated thresholds $n_{+}$and $n_{-}$yield the full FE-EHD phase diagram for Ge and $\mathrm{Si}$ including the effects of metastability and the EHD lifetime, and provide a correct procedure to accurately determine the EHD condensation energy $\phi$ and the surface energy w from threshold data. We also presented calculated limits for the EHD drop radius $R$ vs. 
temperature. These results were obtained for two separate assumptions: homogeneous nucleation, and nucleation of EHD on neutral impurity atoms .

Our experimental results were obtained using an extremely sensitive apparatus described in Chapter 4, specially developed for the study of hysteresis and threshold phenomena in the luminescence of EHD and FE from optically excited Ge. Volume excitation of the Ge samples was used whenever possible to minimize effects of the sample surfaces and to simplify the comparison with nucleation theory. We also made a study of the effect of dislocations and impurity atoms on EHD nucleation by examining a large number (eight) of specially selected ultrapure Ge samples.

High-sensitivity measurements of the hysteresis curves and the metastable time development of the EHD and FE luminescence intensity from Ge between $1.2 \mathrm{~K}$ and $2.1 \mathrm{~K}$ were obtained by cycling a highly stable source of optical excitation in an accurately reproducible manner. The experimental data, presented in Chapter 5, are in good qualitative and quantitive agreement with EHD nucleation theory. In section 51 we use hysteresis data for $E H D$ and $F E$ to obtain an estimate $p \sim 4.4$ for the EHD radiative enhancement factor; this experimental result is in good agreement with Fermi liquid theory. In section 52 we show that hysteresis can exist in the FE-EHD system for times as 1ong as $6 \times 10^{4} \mathrm{sec}$; this fact implies that EHD are practically immobile under suitable circumstances, and has possible technological applications. We also show that the "upgoing" and "downgoing" EHD luminescence 
intensities $\mathrm{I}_{\mathrm{d}}^{+}$and $\mathrm{I}_{\mathrm{d}}^{-}$approach equilibrium logarithmically. The apparent risetime ( $10 \%$ to $90 \%$ of final value) of the EHD signal $\mathrm{I}_{\mathrm{d}}{ }^{+}$ is measured to be as 1ong as $\sim 10^{3} \mathrm{sec}$, a factor $\sim 10^{7}$ longer than that expected from the EHD lifetime $\tau_{0} \approx 40 \mu \mathrm{sec}$ alone. These effects are predicted by nucleation theory and give a striking demonstration of the importance of metastable effects in the EHD-FE system. In Section 53, we show that dislocations are ineffective as nucleation centers for observable EHD, and that EHD observed most probably form on neutral impurity atoms in ultrapure Ge. We also study the temperature dependence of the EHD surface tension $\sigma$ measured from threshold data between $1.5 \mathrm{~K}$ and $2.1 \mathrm{~K}$ and conclude that this dependence is consistent with that expected for a degenerate Fermi 1iquid.

A careful study of the excitation thresholds $\mathrm{P}_{+}$and $\mathrm{P}_{-}$for the creation and destruction of EHD presented in Chapter 5 establishes that these are unique and well-defined quantities for volume excitation. Using an experimental analysis of the $\mathrm{FE}$ luminescence intensity to calibrate the corresponding FE density thresholds $n_{+}$and $n_{-}$, we accurately measure the FE-EHD phase diagram for ultrapure Ge between $1.2 \mathrm{~K}$ and $2.0 \mathrm{~K}$, including the effects of hysteresis and metastability. The experimental data are in excellent quantitative agreement with the phase diagram calculated with EHD nucleation theory. A fit of theory to experiment yields an accurate measurement of the EHD surface energy $w=(2.6 \pm 0.3) \times 10^{-4} \mathrm{erg} \mathrm{cm}^{-2}$, and the first correct measurement of the condensation energy from threshold data, $\phi_{0}=1.9 \pm 0.2 \mathrm{meV}$ at $\mathrm{T}=0$. 
The experimental results described above establish that the EHD-FE system in Ge at low temperatures and levels of excitation is accurately described by EHD nucleation theory. Although hysteresis effects in the luminescence of EHD and FE in Ge seemed highly unusual and even somewhat implausible when they were discovered, particularly because of the very long time scales involved, we have shown that they are a natural consequence of the process of nucleation of electron-hole drops. 


\section{ACKNOWLEDGEMENTS}

I am very grateful to Professor Carson D. Jeffries for the continued advice, support, and encouragement he has given me during the course of this work. I have never worked with a person who was more truly helpful than he has been. I am also grateful to Professor Charles Kittel for many useful comments and suggestions regarding nucleation theory. Dr. Jean Louis Staehli shared the work of constructing the experimental apparatus and recording the early data on hysteresis effects, including the results presented in Section 53 I also owe him thanks for the many hours we spent discussing this work. Dr. William L. Hansen and Dr. Eugene. E. Haller generously provided the crystals of ultrapure Ge used in these experiments and shared their extensive knowledge of crystal defects and impurities. Dr. Haller also constructed the ultra-sensitive Ge photodetector which was essential to the success of this work. I also thank Dr. Robert Marciewicz, Dr. James Wolfe, John Furneaux, Anthony Hansen, Susan Ke1so, and Bernard S. Black for discussions of the theory, apparatus, and results. This work was done under the auspices of the U.S. Energy Research and Development Administration. 
CHAPTER 1 REFERENCES

1.1 L. V. Keldysh, in Proc. IX Int. Conf. on Physics of Semiconductors, Moscow, ("Nauka", Leningrad, 1968), p. 1303.

1.2 Ya. E. Pokrovskii, Phys. Stat. So1. (a) 11, 385 (1972).

1. 3 C. Benoit à 1a Guillaume, M. Voos and F. Salvan, Phys. Rev. B5, 3079 (1972); B 7, 1723 (1973).

1.4 V. S. Bagaev, Springer Tracts in Modern Physics 73, 72 (1975).

1.5 C. D. Jeffries, Science 189, 955 (1975).

1.6 J. C. Hensel, T. G. Phillips and G. A. Thomas, in Solid State Physics, to be published.

1.7 T. M. Rice, in Solid State Physics, to be published.

1.8 M. Lax and J. J. Hopfield, Phys. Rev. 124, 115 (1961).

1.9 G. A. Thomas, M. Capizzi, W. Weber, E. I. Blount and M. Lax, Phys. Rev. Lett. 37, 1000 (1976).

1.10 T. K. Lo, B. J. Feldman and C. D. Jefferies, Phys. Rev. Lett. 31, 224 (1973).

1.11 T. K. Lo, Ph.D. Thesis, University of California, Berkeley, 1973 (unpublished).

1.12 M. Altare11i and N. O. Lipari, Phys. Rev. Lett. 36, 619 (1976); Phys. Rev. B15, 4883 (1977); Phys. Rev. B15, 4898 (1977).

1.13 V. I. Sidorov and Ya. E. Pokrovskii, Fiz. Tech. Poluprov $\underline{6}, 2405$ (1972) [Sov. Phys. Semiconductors 6, 2015 (1973)].

1.14 A. Frova, G. A. Thomas, R. E. Miller and E. O. Kane, Phys. Rev. Lett. $34,1572(1975)$.

1.15 E. 0. Kane, Phys. Rev. B11, 3850 (1975). 
1.16 A. S. Kaminskii and Ya. E. Pokrovskii, Zh. Eksp. Teo. Fiz. Pisma Red. 11, 381 (1970) [JETP Lett. 11, 255 (1970)].

1.17 R. Sauer, Phys. Rev. Lett. 31, 376 (1973).

1.18 K. Kosai and M. Gershenzon, Phys. Rev. B9, 723 (1974).

1.19 R. W. Martin, Solid State Comm. 14, 369 (1974).

1.20 R. Sauer and J. Weber, Phys. Rev. Lett. 36, 48 (1976).

1.21 T. N. Morgan in Proc. XIII Int. Conf. on the Physics of Semiconductors, Rome, ed. by F. G. Fumi (Tipografia Marves, Rome, 1976), p. 825 .

1.22 G. Kirczenow and M. L. W. Thewalt, Bull. Am. Phys. Soc. 22,350 (1977) .

1.23 G. A. Thomas, and T. Rice, Sol. St. Comm. 23, 359 (1977) •

1.24 G. A. Thomas, T. G. Phillips, T. M. Rice and J. C. Hensel, Phys. Rev. Lett. 31, 386 (1973); G. A. Thomas, A. Frova, J. C. Hense1, R. E. Miller and P. A. Lee, Phys. Rev. 13, 1692 (1976).

1.25 T. K. Lo, Solid State Comm. 15, 1231 (1974).

1.26 P. Vashishta, S. G. Das and K. S. Singwi, Phys. Rev. Lett. 33, $911(1974)$.

1.27 G. A. Thomas, T. M. Rice and J. C. Hense1, Phys. Rev. Lett. 33, 219 (1974).

1.28 R. M. Westervelt, T. K. Lo, J. L. Staehli and C. D. Jeffries, Phys. Rev. Lett. 32, 105 (1974); 32, 1331 (E) (1974).

1.29 R. M. Westervelt, J. L. Staehli, E. E. Haller and C. D. Jeffries, in Lecture Notes in Physics vol. 57, ed. by M. Ueta and Y. Nishina (Springer-Verlag, Berlin, 1976) p. 270. 
1.30 R. M. Westervelt, in Proc. XIII Int. Conf. on the Physics of Semiconductors, Rome, ed. by F.G. Fumi (Tipografia Marves, Rome, 1976), p.902.

1.31 J. H. Rose and H. B. Shore, Bu11. Am. Phys. Soc. 21, 223 (1976); and to be published.

1.32 P. Vashishta, R. K. Kalia and K. S. Singwi, in Lecture Notes in Physics, vo1. 57, ed. by M. Ueta and Y. Nishina (Springer-Verlag, Berlin, 1976), p. 187.

1.33 C. Kitte1, Therma1 Physics (John Wiley and Sons Inc., New York, 1969), p. 326 .

1.34 J. C. Hense1, T. G. Phillips and T. M. Rice, Phys. Lett. 30,227 (1973) .

1.35 J. C. McGroddy, M. Voos and O. Christensen, Solid State Comm. 13, 1801 (1973).

1.36 R. M. Westerve1t, J. L. Staeh1i and E. E. Haller, Bull. Am. Phys. Soc. 20, 471 (1975); J. L. Staehli, R. M. Westervelt and E. E. Haller, ibid, p. 471.

1.37 0. Volmer, Z. Phys. Chem. (Leipzig) 119, 277 (1926)

1.38 R. Becker and W. Döring, Ann. Phys. 24, 719 (1935); R. Becker, Theory of Heat, 2nd ed. (Springer-Verlag, New York, 1967) p. 239.

1.39 See J. Frenke1, Kinetic Theory of Liquids (Oxford Univ. Press, Oxford, 1946) p. 390.

1.40 J. E. McDonald, Amer. J. Phys. 30, 870 (1962); 31, 31 (1963).

1.41 F. F. Abraham, Homogeneous Nucleation Theory (Academic Press, New York, 1974). 
1.42 J. Lothe and G. M. Pound, J. Chem. Phys. 36, 2080 (1962).

1..43 R. N. Silver, Phys. Rev. B11, 1569 (1975). 
CHAPTER 2 REFERENCES

2.1 R. M. Westerve1t, Phys. Stat. So1. (b) 74, 727 (1976).

2.2 R. Becker and W. Döring, Ann. Physik 24, 719 (1935); R. Becker, Theory of Heat, 2nd Ed. (Springer-Verlag, New York, 1967), p. 239.

2.3 J. C. Hensel and T. G. Phillips, in Proc. XII Int. Conf. on the Physics of Semiconductors, Stuttgart, M. H. Pilkuhn, Ed. (B. G. Teubner, Stuttgart, 1974), p.51.

2.4 R. S. Markiewicz, Thesis, University of California, Berkeley (unpublished, 1975).

2.5 C. Benoit à 1a Guillaume, M. Voos, and F. Salvan, Phys. Rev. B5, 3079 (1972), and $\underline{B 7}, 1723$ (1973).

2.6 Ya. Pokrovskii, Phys. Stat. So1. (a) 11, 385 (1972).

2.7 T. L. Reinecke and S. C. Ying, Phys. Rev. Lett. 35, 311 (1975).

2.8 T. K. Lo, B. J. Feldman, and C. D. Jeffries, Phys. Rev. Lett. 31, $224(1973)$.

2.9 R. M. Westerve1t, J. L. Staeh11, E. E. Haller, and C. D. Jeffries, in Lecture Notes in Physics, Vo1. 57, ed. by M. Ueta and Y. Nishina (Springer-Verlag, Berlin, 1976), p. 270.

2.10 C. D. Jeffries, Science 189, 955 (1975).

2.11 J. Frenke1, Kinetic Theory of Liquids (Oxford Press, Oxford, 1946), p. 390 .

2.12 R. N. Silver, Phys. Rev. B11, 1569 (1975).

2.13 T. K. Lo, Solid State Comm. 15, 1231 (1974). 


$$
\begin{gathered}
01,19393 \\
-149-
\end{gathered}
$$

2.14 G. A. Thomas, T. M. Rice and J. C. Hensel in Proceedings of the Twelfth International Conference on the Physics of Semiconductors, Stuttgart, 1974, edited by M. H. Pilkuhn (B. G. Teubner, Stuttgart, 1974), p. 105 .

2.15 R. M. Westerve1t, T. K. Lo, J. L. Staeh1i, and C. D. Jeffries, Phys. Rev. Lett. 32, 1051 (1974); (E) 32, 1331 (1974). 
CHAPTER 3 REFERENCES

3.1 R. M. Westervelt, Phys. Stat. So1. (b) 76, 31 (1976).

3.2 R. M. Westervelt, J. L. Staeh1i, E. E. Haller and C. D. Jeffries, in Lecture Notes in Physics, vo1. 57 ed. by M. Ueta and Y. Nishina (Springer-Verlag, Berlin, 1976), p. 270.

3.3 R. M. Westervelt, J. L. Staehli and E. E. Haller, Bull. Am. Phys. Soc. 20, 471 (1975); J. L. Staeh1i, R. M. Westerve1t and E. E. Haller, ibid., p. 471 .

3.4 T. K. Lo, Solid State Comm. 15, 1231 (1974).

3.5 G. A. Thomas, T. M. Rice, and J. C. Hense1, in Proceedings of the Twelfth International Conference on the Physics of Semiconductors, Stuttgart, 1974, edited by M. H. Pilkuhn (B. G. Teubner, Stuttgart, 1974), p. 105 .

3.6 R. M. Westervelt, T. K. Lo, J. L. Staehli and C. D. Jeffries, Phys. Rev. Lett, 32, 1051 (1974); (E) 32, 1331 (1974).

3.7 S. Zwerd1ing, B. Lax, L. M. Roth and K. J. Button, Phys. Rev. 114, 80 (1959).

3.8 Ya. Pokrovskii, Phys. Stat. Sol. (a) 11, 385 (1972).

3.9 G. A. Thomas, T. G. Phillips and J. C. Hense1, Phys. Rev. Lett. 31, 386 (1973); G. A. Thomas, A. Frova, J. C. Hensel, R. E. Miller and P. A. Lee, Phys. Rev.B13, 1692 (1976).

3.10 R. B. Hammond, T. C. McGill and J. W. Mayer, Phys. Rev. B13, 3566 (1976). 
3.11 R. K. Ka1ia and P. Vashishta, Bu11. Am. Phys. Soc. 22, 269 (1977).

3.12 R. W. Martin, Solid State Comm. 14, 369 (1974).

3.13 R. Sauer in Proceedings of the Twelfth International Conference on the Physics of Semiconductors, Stuttgart, 1974, edited by

M. H. Pilkuhn (B. G. Teubner, Stuttgart, 1974), p. 42.

3.14 D. L. Smith, Sol. State Comm. 18, 637 (1976).

3.15 L. M. Sander, H. B. Shore and J. Rose, Bull. Am. Phys. Soc. 22, 269 (1977).

3.16 J. L. Staeh1i, Phys. Stat. Sol. (b) 75, 451 (1976).

3.17 M. Voos, K. L. Shaklee and J. M. Worlock, Phys. Rev. Lett. 33, 1161 (1974).

3.18 B. J. Feldman, Phys. Rev. Lett. 33, 359 (1974).

3.19 C. D. Jeffries, Science 189, 955 (1975).

3.20 T. K. Lo, B. J. Feldman and C. D. Jeffries, Phys. Rev. Lett. 31 , $224(1973)$.

3.21 J. C. McGroddy, M. Voos and 0. Christensen, Solid State Comm. 13, 1801 (1973).

3.22 J. C. Hense1 and T. G. Phillips, in Proc. XII Int. Conf. on the Physics of Semiconductors, Stuttgart, M. H. Pilkuhn, Ed. (B.G. Teubner, Stuttgart, 1974) p. 51.

$3.23 \mathrm{~J}$. Shah, M. Combescot and A. H. Dayem, to be published.

3.24 V. S. Bagaev, N. A. Penin, N. N. Sibel'din and V. A. Tsvetkov, Fiz. Tverd. Tela 15, 3269 (1973) [Sov. Phys. Solid State 15, 2179 (1974)]

3.25 B. Etienne, C. Benoit à la Guillaume and M. Voos, Phys. Rev. Lett. $\underline{35}, 536(1975)$. 
CHAPTER 4 REFERENCES

4.1 Ya. E. Pokrovskii, Physica Stat. Sol.(a) 11, 385 (1972).

4.2 T. K. Lo, B. J. Feldman and C. D. Jeffries, Phys. Rev. Lett. 31 , $224(1973)$

4.3 B. Etienne, C. Benoit à la Guillaume and M. Voos, Phys. Rev. B14, $712(1976)$

4.4 J. L. Staehli, Physica Stat. So1. (b) 75, 451 (1976).

4.5 V. S. Bagaev, N. V. Zamkovets, L. V. Keldysh, N. N. Sibeldin and V. A. Tsuetkov, Zh. Eksp. Teor. Fiz. 70, 1501 (1976).

4.6 W. L. Hansen and E. E. Haller, IEEE Trans. Nuc1. Sci. 21, 251 (1974); E. E. Haller and W. L. Hansen, ibid., p. 279.

4.7 D. L. Smith, So1. State Comm. 18, 637 (1976).

4.8 L. M. Sander, H. B. Shore and J. Rose, Bull. Am. Phys. Soc. 22, 269 (1977).

4.9 E. E. Haller, G. S. Hubbard and W. L. Hansen, IEEE Trans. Nuc1. Sci. NS24, 48 (1977).

4.10 C. Benoit à la Guillaume, M. Voos and F. Salvan, Phys. Rev. B5, 3079 (1972).

4.11 V. S. Bagaev, T. I. Galkina and O. V. Gogolin, Proc. X Int. Conf. on Physics of Semiconductors, Cambridge, 1970, S. P. Keller, J. C. Hense1 and F. Stern eds., CONF-700801 (U.S.A.E.C. Div. of Tech. Info., Springfie1d, Va., 1970), p.500.

4.12 R. G. Rhodes, Imperfections and Active Centers in Semiconductors (MacMillan, New York, 1964), p. 259. 
4.13 W. L. Hansen, private communication.

4.14 R. M. Westerve1t, T. K. Lo, J. L. Staeh1i and C. D. Jeffries, Phys. Rev. Lett. 32, 1051 (1974); 32, 1331 (E) (1974).

4.15 J. C. Hensel, private communication.

4.16 S. M. Sze, Physics of Semiconductor Devices (Wiley-Interscience, New York, 1969), p. 72.

4.17 T. K. Lo, Solid State Comm. 15, 1231 (1974). 


\section{CHAPTER 5 REFERENCES}

5.1 T. K. Lo, B. J. Feldman and C. D. Jeffries, Phys. Rev. Lett. 31, 224 (1973) .

5.2 V. S. Bagaev, L. V. Keldysh, N. N. Sibeldin and V. A. Tsvetkov, Zh. Eksp. Teor. Fiz. 70, 702 (1976).

5.3 L. M. Sander, H. B. Shore and J. Rose, Bull. Am. Phys. Soc. 22, 269 (1977).

5.4 R. M. Westerve1t, T. K. Lo, J. L. Staeh1i and C. D. Jeffries, Phys. Rev. Lett. 32, 1051 (1974); 32, 1331(E) (1974).

5.5 C. Benoit à la Guillaume, F. Salvan and M. Voos, in Proc. X Int. Conf. on the Physics of Semiconductors, Cambridge, S. P. Keller, J. C. Hensel and F. Stern, Eds. (U.S.A.E.C., 1970), p.516.

5.6 G. A. Thomas, A. Frova, J. C. Hense1, R. E. Mi11er and P. A. Lee, Phys. Rev. 13, 1692 (1976).

5.7 P. Vashishta, S. G. Das and K. S. Singwi, Phys. Rev. Lett. 33, $911(1974)$.

5.8 Ya. E. Pokrovskii, Phys. Stat. Sol. (a) 11, 385 (1972).

5.9 R. M. Westerve1t, J. L. Staeh1i, E. E. Haller and C. D. Jeffries, in Lecture Notes in Physics, Vo1. 57, ed. by M. Ueta and Y. Nishina (Springer-Verlag, Berlin, 1976), p. 270 .

5.10 J. L. Staeh1i, Phys. Stat. Sol. (b) 75, 451 (1976).

5.11 J. Shah, A. H. Dayem and M. Combescot, to be published.

5.12 R. M. Westervelt and B. S. Black, Bull. Am. Phys. Soc. 22, 269 (1977); and to be published. 
5.13 J. C. Hensel, T. G. Phillips and G. A. Thomas, Solid State Physics, to be published.

5.14 G. A. Thomas, T. M. Rice and J. C. Hense1, Phys. Rev. Lett. 33, $219(1974)$.

5.15 T. L. Reinecke and S. C. Ying, Phys. Rev. Lett. 35, 311 (1975); 35, 547(E) (1975).

5.16 R. M. Westerve1t, in Proc. XIII Int. Conf. on the Physics of Semiconductors, Rome, 1976, F. G. Fumi, ed. (Tipografia Marves, Rome, 1976), p. 902 .

5.17 J. C. Hense1, T. G. Phillips and T. M. Rice, Phys. Rev. Lett. 30, 227 (1973).

5.18 J. C. McGroddy, M. Voos and 0. Christensen, Solid State Comm. 13, 1801 (1973).

5.19 T. K. Lo, Solid State Comm. 15, 1231 (1974).

5.20 G. A. Thomas, A. Frova, J. C. Hense1, R. E. Miller and P. A. Lee, Phys. Rev. 13, 1692 (1976).

5.21 E. M. Gershenzon, G. N. Goltsman and N. G. Ptitsina, Zh. Eksp. Teor. Fiz. 70, 224 (1976).

5.22 T. Timusk, Phys. Rev. B13, 3511 (1976).

5.23 R. M. Westerve1t, J. L. Staeh1i and E. E. Haller, Bull. Am. Phys. Soc. 20, 471 (1975); J. L. Staeh1i, R. M. Westervelt and E. E. Haller, ibid.

5.24 R. N. Silver, Phys. Rev. B11, 1569 (1975); and B12, 5689 (1975).

5.25 B. Etienne, C. Benoit à la Guillaume and M. Voos, Phys. Rev. B14, $712(1976)$. 
5.26 K. R. E1liot, D. L. Smith and T. C. McGi11, Bul1. Am. Phys. Soc. 22, 350 (1977).

5.27 Ya. E. Pokrovskii and K. I. Svistunova, Fiz. Tverd. Tela. 13, 1485 (1971) [Sov. Phys. - Solid State 13, 1241 (1971)].

5.28 R. Ito, H. Kawamura and M. Fukai, Physics Letters 13, 26 (1964);

J. C. Hense 1 and K. Suzuki, Phys. Rev. B9, 4219 (1974).

5.29 J. C. Hensel and T. G. Phillips, in Proc. XII Int. Conf. on the Physics of Semiconductors, Stuttgart, M. H. Pilkuhn, ed.

(B. G. Teubner, Stuttgart, 1974), p.51.

5.30 M. Altarelli and N. O. Lipari, Phys. Rev. Lett. 36, 619 (1976).

5.31 A. F. Dite, V. D. Kulakovsky and V. B. Timofeev, Zh. Eksp. Teor. Fiz 72, 1156 (1977).

5.32 P. Vashishta, S. G. Das and K. S. Singwi, Phys. Rev. Lett. 31, $911(1974)$.

5.33 T. M. Rice, Phys. Rev. B9, 1540 (1974).

5.34 L. M. Sander, H. B. Shore and L. J. Sham, Phys. Rev. Lett. 31, $533(1973) ; 31,1230(E)(1973)$.

5.35 H. Büttner and E. Gerlach, J. Phys. C 6. L433 (1973).

5.36 T. L. Reinecke and S. C. Ying, Phys. Rev. Lett. 35, 311 (1975); 35, $547(\mathrm{E})(1975)$.

5.37 P. Vashishta, R. K. Kalia and K. S. Singwi, in Lecture Notes in Physics, vol. 57 (Springer-Verlag, Berlin, 1976), p.187.

$5.38 \mathrm{~J} . \mathrm{H}$. Rose and H. B. Shore, to be published.

5.39 V. S. Bagaev, N. N. Sibeldin and V. A. Tsvetkov, ZhETF Pis. Red. 21, 180 (1975) [JETP Lett. 21, 80 (1975)]. 
$-157-$

5.40 B. Etienne, L. M. Sander, C. Benoit à la Guillaume, M. Voos and J. Y. Prieur, Phys. Rev. Lett. 37, 1299 (1977) .

5.41 B. Etienne, C. Benoit à 1a Guillaume and M. Voos, Phys. Rev. Lett. 35, 536 (1975). 
Table 3.1: Values of the electron-hole drop (EHD) and free exciton (FE) parameters used in the calculation (see text).

\begin{tabular}{|c|c|c|c|c|c|c|}
\hline Parameter & Symbo1 & & Ge & $\operatorname{Ref}$. & Si & $\operatorname{Ref}$ \\
\hline surface tension & $\sigma\left(10^{-4}\right.$ erg $\left.\mathrm{cm}^{-2}\right)$ & 2.7 & $-0.064 \mathrm{~T}^{2}$ & $3.2,3.3$ & 80 & - \\
\hline condensation energy & $\phi(\mathrm{K})$ & 22.8 & $+0.19 \mathrm{~T}^{2}$ & $3.4,3.5$ & 79 & 3.8 \\
\hline EHD pair density & $\mathrm{n}_{\mathrm{o}}\left(10^{17} \mathrm{~cm}^{-3}\right)$ & 2.38 & $-0.025 \mathrm{~T}^{2}$ & 3.4 & 37 & 3.8 \\
\hline EHD Iifetime & $\tau_{0}(\mu s e c)$ & & 41 & 3.6 & 0.2 & 3.8 \\
\hline FE lifetime & $\tau_{x}(\mu s e c)$ & & 7.7 & 3.6 & 2 & 3.8 \\
\hline $\mathrm{FE}$ effective mass & $m *\left(m_{0}\right)$ & & 0.335 & 3.7 & 0.60 & 3.7 \\
\hline FE degeneracy & $\gamma_{x}$ & & 16 & 3.7 & 24 & 3.7 \\
\hline impurity concentration & $\mathrm{N}_{i}\left(\mathrm{~cm}^{-3}\right)$ & & $\times 10^{11}$ & - & $1 \times 10^{12}$ & - \\
\hline maximum generation rate & $G_{M}\left(\mathrm{~cm}^{-3} \mathrm{sec}^{-1}\right)$ & & $\times 10^{19}$ & - & $1 \times 10^{21}$ & - \\
\hline observation time & $\Delta t(\mathrm{sec})$ & & 50 & - & 50 & - \\
\hline barrier lowering & $\Delta \psi(\mathrm{K})$ & & 50 & - & 100 & - \\
\hline
\end{tabular}




\section{8}

$-159-$

Table 4.1. Connections between the timing logic and the Northern Scientific mode1 NS-905 multi-channel analyzer (MCA); the signal names are those of the MCA.

\begin{tabular}{lcc}
\hline Signal & Timing Logic Pin & MCA Pin \\
\hline$\overline{\mathrm{EP}}$ & $\mathrm{D}$ & $\mathrm{Y}$ \\
$\overline{\mathrm{EADD}}$ & $\mathrm{A}$ & $\mathrm{S}$ \\
$\overline{\mathrm{ESUB}}$ & $\mathrm{B}$ & $\mathrm{U}$ \\
$\mathrm{GND}$ & $\mathrm{H}$ & $\mathrm{NN}$ \\
\end{tabular}


Table 4.2 List of Ge samples and characteristics

\begin{tabular}{|c|c|c|c|c|c|c|c|c|c|c|}
\hline \multirow{2}{*}{$\begin{array}{l}\text { Sample } \\
\text { number }\end{array}$} & \multirow{2}{*}{$\frac{\begin{array}{c}\text { size } \\
\left(\mathrm{mm}^{3}\right)\end{array}}{4 \times 4 \times 9}$} & \multirow{2}{*}{ boule } & \multirow{2}{*}{$\frac{\operatorname{cut}(\mathrm{cm} .)}{-0.4}$} & \multirow{2}{*}{$\underbrace{\text { face }}_{\langle 100\rangle}$} & \multirow{2}{*}{ type } & \multicolumn{2}{|c|}{ density $\left(\mathrm{cm}^{-3}\right)$} & \multirow{2}{*}{$\frac{\operatorname{density}\left(\mathrm{cm}^{-2}\right)}{\sim 10^{4}}$} & \multicolumn{2}{|c|}{$\tau_{x}($ usec $)$} \\
\hline & & & & & & 3 & $\times 10^{9}$ & & $3.5^{\mathrm{a}}$ & $3.8^{b}$ \\
\hline $150 D$ & $4 \times 3 \times 9$ & 150 & 10.3 & $\langle 100\rangle$ & $\mathrm{p}$ & 5 & $\times 10^{10}$ & $10^{2}$ & $6.9^{\mathrm{a}}$ & $10.4^{\mathrm{b}}$ \\
\hline $150 \mathrm{~V}$ & $4 \times 3 \times 9$ & 150 & 10.3 & $\langle 100\rangle$ & $\mathrm{p}$ & 3 & $\times 10^{11}$ & 0 & $11.4^{\mathrm{a}}$ & \\
\hline 145 & $4 \times 3 \times 9$ & 145 & $?$ & $\langle 100\rangle$ & $\mathrm{p}$ & 2 & $\times 10^{11}$ & 0 & & $7.7^{\mathrm{b}}$ \\
\hline 391 & $4 \times 3 \times 9$ & 391 & 2.0 & $\langle 111\rangle$ & $\mathrm{p}$ & & $\times 10^{11}$ & $10^{4}$ & & \\
\hline 393 & $4 \times 3 \times 9$ & 393 & 3.0 & $\langle 111\rangle$ & $\mathrm{n}$ & & $\times 10^{11}$ & $10^{4}$ & & \\
\hline 400 & $4 \times 3 \times 9$ & 400 & 3.0 & $\langle 111\rangle$ & $\mathrm{n}$ & & $\times 10^{12}$ & $10^{2}$ & & $14.3^{b}$ \\
\hline 394 & $4 \times 3 \times 9$ & 394 & 2.1 & $\langle 111\rangle$ & $\mathrm{n}$ & 6 & $\times 10^{10}$ & $10^{4}$ & $9.4^{\mathrm{a}}$ & \\
\hline 356 & $3 \times 18 \times 20$ & 356 & 1.6 & $\langle 100\rangle$ & $\mathrm{p}$ & & $\times 10^{10}$ & $10^{3}$ & & \\
\hline
\end{tabular}


Table 5.1. Measured threshold ratios $P_{+} / P_{-}$and calculated EHD surface tension $\sigma$ for homogeneous nucleation (Homo.) and nucleation on impurities for three values of the barrier lowering

$\Delta \psi$ (see Chapter 2). For more sample characteristics see Table 4.2. Impurity type d denotes $80 \mathrm{meV}$ acceptor levels associated with vacancy clusters.

\begin{tabular}{|c|c|c|c|c|c|c|c|c|c|}
\hline \multirow[t]{2}{*}{ Sample } & \multicolumn{2}{|c|}{ Impurity } & \multirow{2}{*}{$\begin{array}{l}\text { Dislocation } \\
\text { Density }\left(\mathrm{cm}^{-2}\right)\end{array}$} & \multirow[t]{2}{*}{$T(K)$} & \multirow{2}{*}{$P_{+} / P_{-}$} & \multicolumn{4}{|c|}{ Surface Tension $\sigma\left(10^{-4}\right.$ erg $\left.\mathrm{cm}^{-2}\right)$} \\
\hline & Type & Density $\left(\mathrm{cm}^{-3}\right)$ & & & & Homo. & $\Delta \psi=50 \mathrm{~K}$ & $100 \mathrm{~K}$ & $200 \mathrm{~K}$ \\
\hline \multirow[t]{2}{*}{315} & $\mathrm{n}$ & $3 \times 10^{9}$ & $\sim 10^{4}$ & 2.10 & 3.8 & 2.5 & 2.5 & 2.9 & 3.4 \\
\hline & & & & 2.10 & 4.3 & 2.7 & 2.6 & 3.0 & 3.5 \\
\hline $150 \mathrm{D}$ & $\mathrm{p}$ & $5 \times 10^{10}$ & $\sim 10^{2}$ & 2.07 & 3.3 & 2.4 & 2.4 & 2.5 & 3.0 \\
\hline $150 \mathrm{~V}$ & d & $3 \times 10^{11}$ & $\sim 0$ & 2.06 & 2.7 & 2.2 & 2.2 & 2.5 & 3.0 \\
\hline 145 & $\mathrm{~d}$ & $2 \times 10^{11}$ & $\sim 0$ & 2.04 & 3.4 & 2.4 & 2.5 & 2.8 & 3.3 \\
\hline \multirow[t]{2}{*}{393} & $\mathrm{n}$ & $1 \times 10^{11}$ & $\sim 10^{3}$ & 2.09 & 2.8 & 2.2 & 2.2 & 2.5 & 3.0 \\
\hline & & & & 2.09 & 2.8 & 2.2 & 2.2 & 2.5 & 3.0 \\
\hline \multirow[t]{2}{*}{391} & $\mathrm{p}$ & $2 \times 10^{11}$ & $\sim 10^{4}$ & 2.10 & 3.2 & 2.4 & 2.4 & 2.7 & 3.2 \\
\hline & & & & 2.09 & 3.0 & 2.3 & 2.3 & 2.6 & 3.1 \\
\hline 400 & $\mathrm{n}$ & $4 \times 10^{12}$ & $\sim 10^{2}$ & 2.09 & 2.5 & 2.1 & 2.1 & 2.4 & 2.8 \\
\hline
\end{tabular}


Table 5.2 Theoretical and experimental values for the EHD surface tension $\sigma$ and the surface energy $w=\sigma$ at $T=0$.

Theory

$\begin{array}{clc}\mathrm{w}\left(10^{-4} \mathrm{erg} \mathrm{cm}-2\right) & \text { Ref. } \\ 0.8 & \text { Ruthors } & 5.33 \\ 1 & \text { Rice } & 5.34 \\ 0.8 \text { to } 2.2 & \text { Büder et al. } & 5.35 \\ 1.3 & \text { Reinecke and Ying } & 5.36 \\ 3.5 \text { to } 4 & \text { Vashishta et al. } & 5.37 \\ 2.4 \pm 0.5 & \text { Rose et a1. } & 5.38\end{array}$

\section{Experiment}

\begin{tabular}{|c|c|c|c|}
\hline$\sigma\left(10^{-4}\right.$ erg $\left.\mathrm{cm}^{-2}\right)$ & $\mathrm{T}(\mathrm{K})$ & Authors & Ref. \\
\hline 1.8 & $\sim 2.5$ & Bagaev et al. & 5.39 \\
\hline 2.4 to 2.8 & 0 & Westerve1t et a1. & $5.9,5.23$ \\
\hline 1.8 to 2.6 & $\sim 3$ & Etienne et al. & 5.25 \\
\hline$\sim 3$ & 1.8 & Etienne et a1. & 5.40 \\
\hline 2.6 & 2.1 & This work, Section 53 & \\
\hline $2.6 \pm 0.3$ & 0 & This work & 5.16 \\
\hline
\end{tabular}


Fig. 1.1 Schematic variation (not to scale) of the valence and conduction energy bands of Ge with wavevector along the $(1,1,1)$ direction. The lowest minimum in the conduction bond lies at the Brillioun zone boundary $k=(2 \pi / a)\left(\frac{1}{2}, \frac{1}{2}, \frac{1}{2}\right)$. An empty band is shown for free excitons (FE), and partially filled bands for electronhole drops (EHD). The FE binding energy $E_{x}$, and the electron and hole work functions $\phi_{\mathrm{e}}$ and $\phi_{\mathrm{h}}$ and Fermi energies $\mathrm{E}_{\mathrm{F}}{ }^{\mathrm{e}}$ and $\mathrm{E}_{\mathrm{F}}^{\mathrm{h}}$ for EHD are indicated. Note that the electron Fermi leve1 has been drawn lower than $\mathrm{E}_{\mathrm{x}}$ for clarity.

Fig. 1.2 Luminescence spectrum of $\mathrm{EHD}$ and $\mathrm{FE}$ in ultrapure Ge (boule 315) at $2.00 \mathrm{~K}$ excited by the focussed beam of a Ar-Kr ion laser (absorbed power $40 \mathrm{~mW}$ ). The apparatus used to record this data and that of Figs. 1.3 and 1.4 is essentially that described in chapter 4, with the exception that the exciting light is chopped to avoid hysteresis effects. The spectral resolution (FWHM) is indicated. The wavelength was calibrated with the $435.835 \mathrm{~nm}$ line from a $\mathrm{Hg}$ germicidal lamp in $4 \mathrm{th}$ order.

Fig. 1.3 Luminescence spectrum of FE in ultrapure Ge (boule 315) at 2.10K surface excited below the threshold for EHD formation by the chopped light from a tungsten lamp. Two phonon replicas, LA and TO, are indicated. 
Fig. 1.4. Luminescence spectrum of EHD and $\mathrm{FE}$ in ultrapure Ge (boule 315) at $2.10 \mathrm{~K}$ excited above the threshold for drop formation y. The excitation power is 2.2 times that for Fig. 1.3, otherwise the conditions for Figs. 1.3 and 1.4 are identical. The LA phonon replicas for $\mathrm{EHD}$ and $\mathrm{FE}$ are labelled EHD and FE respectively; the small bump on the low energy side of the EHD line is the TO phonon replica for FE.

Fig. 1.5. Free energy difference $\Delta$ f required to create an embryonic EHD vs. drop radius $\mathrm{R}$ from Eq. (1.6) for water at 300K. Plotted for values of the supersaturation ratio $\mathrm{n} / \mathrm{n}_{\mathrm{s}}$ : a) 1, b) 2 , c) 5 , and d) 10 . The critical radius $R_{c}$ and the potential barrier $\Delta \mathrm{f}_{\mathrm{c}}$ are indicated for curve $\left.\mathrm{b}\right)$. The value $\sigma=70 \mathrm{erg} \mathrm{cm}^{-2}$ was used for the surface tension of water (see Ref. 1.39). 


\section{CHAPTER 2 FIGURE CAPTIONS}

Fig. 2.1 Effective free energy difference $\psi_{\nu}$ at $2 \mathrm{~K}$ computed from Eq. (2.17) for EHD in Ge versus drop radius for various values of the degree of supersaturation $\mathrm{x}$ from Eq. (2.9): a) The minimum value $\mathrm{x}_{\mathrm{m}}=0.728, \mathrm{~b}$ ) the threshold value for drop breakup $x_{-}=0.734$, c) the threshold value for significant equilibrium drop population $\left.x_{e}=0.771, d\right) x=0.913$, and e) the threshold value for drop formation $x_{+}=2.01$. The values of the parameters used (see text) are $\sigma=2.7 \times 10^{-4}\left[1-(\mathrm{T} / 6.5)^{2}\right] \operatorname{erg~cm}^{-2}(\operatorname{Ref} .2 .9)$, $\phi=\left(22.8+0.19 \mathrm{r}^{2}\right) \mathrm{K}$ the value of Ref. 2.13 corrected for the temperature dependence reported in Ref. 2.14, $\mathrm{n}_{\mathrm{o}}=\left(2.38-0.025 \mathrm{~T}^{2}\right) \times 10^{17} \mathrm{~cm}^{-3}\left(\right.$ Ref. 2.13), $\mathrm{m} *=0.335 \mathrm{~m}_{\mathrm{o}}$ $(\operatorname{Ref} .2 .8), \gamma_{\mathrm{X}}=16(\operatorname{Ref} .2 .8)$, and $\tau_{\mathrm{o}}=41 \mu \mathrm{sec}(\operatorname{Ref} \cdot 2.15)$.

Fig. 2.2 Effective free energy difference $\psi_{V}$ at $2 \mathrm{~K}$ as in Fig. 2.1, replotted logarithmica11y. The letters refer to the same values of the degree of supersaturation used in Fig. 2.1, and the values used for the parameters are the same. Note the inversion and break in the vertical logarithmic scale at $\psi=10 \mathrm{~K}$.

Fig. 2.3 Homogeneous rate of formation $\mathrm{J}_{+}$and breakup $\mathrm{J}_{-}$of macroscopic EHD for Ge computed from Eqs. (2.24) and (2.25) at various temperatures, versus the degree of supersaturation $\mathrm{x} \equiv \ln \left(\mathrm{n} / \mathrm{n}_{\mathrm{s}}\right)$ of the $\mathrm{FE}$ gas. The parameters used are those 
of Fig. 2.1, and the initial EHD concentration $N_{\text {for }} \mathrm{J}_{-}$is chosen such that $N_{-}=4.1 \times 10^{14} \mathrm{~cm}^{-3}$ where $\nu_{-}$is the number of e-h pairs in a drop at the threshold for drop breakup. These values for $\mathrm{N}$ assume a constant initial $\mathrm{e}-\mathrm{h}$ pair generation rate. 
Fig. 3.1 Electron-hole drop (EHD) signal NV versus generation rate $G$ for Ge assuming homogeneous nucleation. The curves labeled UP and DOWN are for monotonically increasing and decreasing $G$ to the value indicated (see text). Inserts (c) and (b) illustrate the behavior of $N$ V near the thresholds $G_{+}$and $G_{-}$ defined in the text.

Fig. 3.2 Value of the EHD surface tension $\sigma$ at various temperatures versus the threshold ratio $G_{+} / G_{-}$for Ge assuming (a) homogeneous nucleation and (b) inhomogeneous nucleation. The values of parameters other than $\sigma$ are given in Table 3.1. These graphs can be used to determine $\sigma$ from threshold measurements .

Fig. 3.3 EHD-free exciton (FE) phase diagram for Ge including the effects of metastability and the EHD 1ifetime, for homogeneous and inhomogeneous nucleation of drops. In both graphs (a) is the up-going threshold, (b) the down-going threshold, and (c) the "saturated" FE density (see text). Where (a) and (b) do not differ the system is in dynamic equilibrium, and the curve shown is the equilibrium threshold (see text).

Fig. 3.4 FE-EHD phase diagram in Ge for various values of the possible temperature-independent evaporation coefficient:
a) $c=0 \mathrm{sec}^{-1}$ redrawn from $\left.\mathrm{Fig} \cdot 3.3, \mathrm{~b}\right) \mathrm{c}=5 \times 10^{4} \mathrm{sec}^{-1}$,
c) $c=5 \times 10^{5} \mathrm{sec}^{-1}$, and d) $c=5 \times 10^{6} \mathrm{sec}^{-1}$. 
Fig. 3.5 FE-EHD phase diagram for $\mathrm{Si}$ analogous to Fig. 3.3. In both graphs the curves are (a) the up-going threshold, (b) the down-going threshold, and (c) the "saturated" FE density (see text). Where (a) and (b) are the same, the curve is the equilibrium threshold (see text).

Fig. 3.6 Predicted EHD radius near threshold versus temperature for Ge assuming (a) homogeneous and (b) inhomogeneous nucleation. The curves labeled UP and DOWN are for monotonically increasing and decreasing the excitation. Above $\mathrm{T} \sim 2.5 \mathrm{~K}$ the diffusion limited radius $\mathrm{R}_{\mathrm{D}}$ calculated from Eqs. (3.21) and (3.22), shown as the dashed curves, should be observed rather than the up-going (UP) radius. 
Fig. 4.1 Schematic diagram of the apparatus used for volume excitation and detection of the luminescence of EHD and FE in Ge. The inserts show the cycles of excitation used and are discussed in the text.

Fig. 4.2 Excitation spectrum for volume excitation. The background level is partially limited by the spectrometer used to take this data and is not necessarily leakage through the interference filter.

Fig. 4.3 Block diagram of the feedback-stabilized lamp contro1 electronics (enclosed within the dotted lines) showing the connections to the Ge monitor detector, the programmable DC lamp power supply, and the multi-channel analyzer (MCA).

Fig. 4.4 Schematic circuit diagrams for various components of the excitation cycling electronics: a) reference function generator, b) current amplifier, c) differential amplifier.

Fig. 4.5 Circuit diagram for the timing and control logic of the excitation cycling electronics. The signals $\overline{\mathrm{EP}}, \overline{\mathrm{EADD}}$, and $\overline{\text { ESUB }}$ interface the circuit to a Northern Scientific NA-900A multi-channe1 analyzer.

Fig, 4.6 Diagram of the sample holder and method of mounting. The Ge sample is electrically and mechanically isolated from both the $\mathrm{Cu}$ plate and the wire springs by $0.010^{\prime \prime}$ Mylar pads as indicated. 
Fig. 4.7 Wavelength response of the Ge detector to tungsten-1amp illumination for various detector temperatures: a) $158 \mathrm{~K}$, b) $166 \mathrm{~K}$, and c) $172 \mathrm{~K}$. The peak positions of the LA phonon assisted 1uminescence of $\mathrm{FE}$ and $\mathrm{EHD}$ are indicated.

Fig. 4.8 Circuit diagram of the sensitive current amplifier, and the biasing connections for the Ge luminescence detector.

Fig. 4.9 Circuit diagram of the selective clipper used to remove fast gamma ray noise spikes from the preamplifier output. 


\section{0,44004964

CHAPTER 5 FIGURE CAPTIONS

Fig. 5.1 Logarithmic plot for sample 315 (see Table 4.2) of the EHD and FE luminescence intensity vs. absorbed surface excitation power $\mathrm{P}$ at $2.10 \mathrm{~K}$ obtained by increasing (UP) and by decreasing (DOWN) $P$ to the level indicated as described in the text. The initial excitation in the downgoing case is $\mathrm{P}_{\max }=$ $0.90 \mathrm{~W} \mathrm{~cm}^{-2}$.

Fig. 5.2 Hysteresis curves $\mathrm{I}_{\mathrm{d}}{ }^{ \pm}$and $\mathrm{I}_{\mathrm{x}}{ }^{ \pm}$for $\mathrm{EHD}$ and $\mathrm{FE}$ in sample 150D

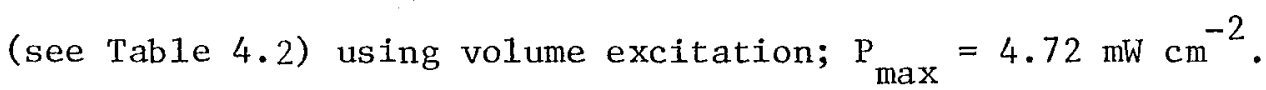
The intensity scale for $\mathrm{FE}$ is expanded by a factor of two; the excitation thresholds $\mathrm{P}_{+}$and $\mathrm{P}_{-}$are indicated.

Fig. 5.3 Family of hysteresis curves for EHD in sample 315 (see Table 4.2) using volume excitation, for various values of $\mathrm{P}_{\max }$ in the downgoing case as indicated. The thresholds $\mathrm{P}_{+}$ and $\mathrm{P}_{\text {_ }}$ are shown, and the critical excitation $\mathrm{P}_{\mathrm{C}}$ is indicated for curve (b) (see text).

Fig. 5.4 Theoretical hysteresis curves for EHD calculated using nucleation theory as described in the text, which can be directly compared with Fig. 5.3, for various values of the initial generation rate $G_{M}$ in the downgoing case expressed in units $10^{17} \mathrm{~cm}^{-3} \mathrm{sec}^{-1}$.

Fig. 5.5 EHD luminescence intensity obtained by using the cycle of excitation shown in the inset as described in the text. The signal is recorded during the time interval labelled "add". For sample 315 (see Table 4.2) using volume excitation. The 
threshold $\mathrm{P}_{\text {_ }}$ and the critical excitation $\mathrm{P}_{\mathrm{C}}$ are indicated. This figure is to be compared with Fig. 5.3.

Fig. 5.6 Hysteresis curves for EHD in sample 315 (see Table 4.2) at $1.86 \mathrm{~K}$ using volume excitation; $\mathrm{P}_{\max }=4.72 \mathrm{~mW} \mathrm{~cm} \mathrm{~cm}^{-2}$. The thresholds $\mathrm{P}_{+}$and $\mathrm{P}_{-}$extrapolated from the data as described in the text are indicated.

Fig. 5.7 Hysteresis curves for EHD in sample 315 (see Table 4.2) at $1.64 \mathrm{~K}$ using volume excitation; $\mathrm{P}_{\max }=4.74 \mathrm{~mW} \mathrm{~cm}^{-2}$. The extrapolated thresholds $\mathrm{P}_{+}$and $\mathrm{P}_{-}$are indicated.

Fig. 5.8 Hysteresis curves for EHD in sample 315 (see Table 4.2) at $1.45 \mathrm{~K}$ using volume excitation; $\mathrm{P}_{\max }=4.72 \mathrm{~mW} \mathrm{~cm}{ }^{-2}$. The extrapolated thresholds $\mathrm{P}_{+}$and $\mathrm{P}_{-}$are indicated.

Fig. 5.9 Hysteresis curves for EHD in sample 315 (see Table 4.2) at $1.25 \mathrm{~K}$ using volume excitation; $\mathrm{P}_{\max }=1.58 \mathrm{~mW} \mathrm{~cm}{ }^{-2}$. The extrapolated thresholds $\mathrm{P}_{+}$and $\mathrm{P}_{-}$accurately coincide as indicated.

Fig. 5.10 Replot of the data of Fig. 5.7 illustrating the procedure used to extrapolate the thresholds $\mathrm{P}_{+}$and $\mathrm{P}_{-}$from the square root of the EHD luminescence intensity $\mathrm{I}_{\mathrm{d}}$.

Fig. 5.11 Metastable time development of the EHD signal $\mathrm{I}_{\mathrm{d}}$ at $1.80 \mathrm{~K}$ for sample 315 (see Table 4.2) using volume excitation; for both curves $\mathrm{P}=2.31 \mathrm{~mW} \mathrm{~cm}{ }^{-2} ; \mathrm{P}_{\max }=4.72 \mathrm{~mW} \mathrm{~cm}^{-2}$. Note the suppressed zero of $I_{d}$ in both curves and the logarithmic time scale for the lower curve. The solid line for the upper curve is an exponential fit to the data for $\Delta t \geqslant 10^{3} \mathrm{sec}$ yielding the time constant $\tau$ indicated. 
Fig. 5.12 Metastable time development of the EHD signal at $1.50 \mathrm{~K}$ for sample 315 (see Table 4.2) using volume excitation; for both

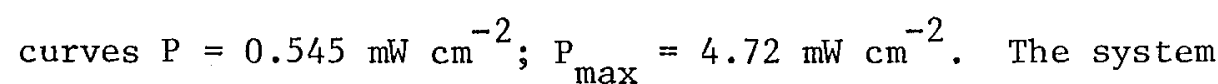
reaches dynamic equilibrium at $\Delta t \approx 10^{2} \mathrm{sec}$.

Fig. 5.13 Metastable time development of the downgoing EHD signal near the threshold $P_{\text {_ }}$ for the destruction of EHD for sample 315 (see Table 4.2) using volume excitation; $\mathrm{P}=0.437 \mathrm{~mW} \mathrm{~cm} \mathrm{~cm}^{-2}$; $\mathrm{P}_{\max }=4.72 \mathrm{~mW} \mathrm{~cm}{ }^{-2}$. The EHD signal disappears at $\Delta t \approx 200 \mathrm{sec}$.

Fig. 5.14 Time development of the EHD signa1 at $1.25 \mathrm{~K}$ for sample 315 (see Table 4.2) using volume excitation; for both curves $\mathrm{P}=0.370 \mathrm{~mW} \mathrm{~cm} \mathrm{~cm}^{-2} ; \mathrm{P}_{\max }=4.72 \mathrm{~mW} \mathrm{~cm}^{-2}$. Note the suppressed zeros in the EHD signal.

Fig. 5.15 Plot of the logarithm of the excitation threshold ratio $\mathrm{P}_{+} / \mathrm{P}_{-}$measured for eight different crystals of ultrapure Ge vs. approximate dislocation etch pit density. The sample numbers and dominant impurity type are indicated (see Table 4.2); type d denotes $80 \mathrm{meV}$ acceptor levels associated with vacancy clusters in undislocated crystals.

Fig. 5.16 Replot of the data of Fig. 5.15 vs. net impurity concentration $\left|\mathrm{N}_{\mathrm{A}}-\mathrm{N}_{\mathrm{D}}\right|$ for the dislocated samples. The dashed curve is intended only to guide the eye.

Fig. 5.17 Value of the EHD surface tension $\sigma$ vs. $\mathrm{T}^{2}$ deduced from measured threshold ratios $\mathrm{P}_{+} / \mathrm{P}_{\text {- }}$ for sample 315 (see Table 4.2) for three separate assumptions as indicated and described in the text. The straight solid lines are least-squares fits to the data. 
Fig. 5.18 Hysteresis curves for EHD in sample 315 (see Table 4.2) at $1.82 \mathrm{~K}$ using surface excitation. First the data for Run 1 were recorded (elapsed time $\approx 1$ hour), then the data for Run 2 (see text); note the lack of reproducibility. The effective excitation power is corrected for the ratio 1.8 of the mean photon energies $h \nu$ for surface and volume excitation so that this scale corresponds to that shown in Fig. 5.19 for the latter case; in these units $\mathrm{P}_{\max }=$ $7.70 \mathrm{~mW} \mathrm{~cm}^{-2}$.

Fig. 5.19 Hysteresis curves for EHD in sample 315 (see Table 4.2) at $1.82 \mathrm{~K}$ as in Fig. 5.18 except for volume excitation; $\mathrm{P}_{\max }=4.69 \mathrm{~mW} \mathrm{~cm}^{-2}$. Note the excellent reproducibility.

Fig. 5.20 Constant excitation contours for the FE luminescence intensity vs. $1 / T$ below the threshold for drop formation for sample 315 (see Table 4.2) using volume excitation. The excitation power $\mathrm{P}$ for each curve is indicated; successive values of $\mathrm{P}$ each differ by a factor $\approx 2.0$.

Fig. 5.21 FE 1uminescence intensity vs. excitation power $P$ below the threshold for EHD formation in sample 315 (see Table 4.2) at $2.00 \mathrm{~K}$ using volume excitation. The straight solid line is a least-squares fit to the data for $\mathrm{P}>0.7 \mathrm{~mW} \mathrm{~cm}{ }^{-2}$; note the non-linearity at low excitation levels.

Fig. 5.22 Threshold FE-EHD phase diagram between $1.2 \mathrm{~K}$ and $2.0 \mathrm{~K}$ for sample 315 (see Table 4.2) obtained using volume excitation. The measured data points are proportional to the $\mathrm{FE}$ 
luminescence intensity at threshold. The solid lines are the results of a least-squares fit of the entire phase diagram to the FE density thresholds $n_{+}$and $n_{-}$calculated using EHD nucleation theory (see text). The FE density scale shown is the theoretical one, the calibrated measurements of the threshold density are $\approx 4$ times smaller. The dashed curve is the theoretical saturated FE density $n_{s}$ which describes the phase diagram for ordinary liquids in thermodynamic equilibrium. 


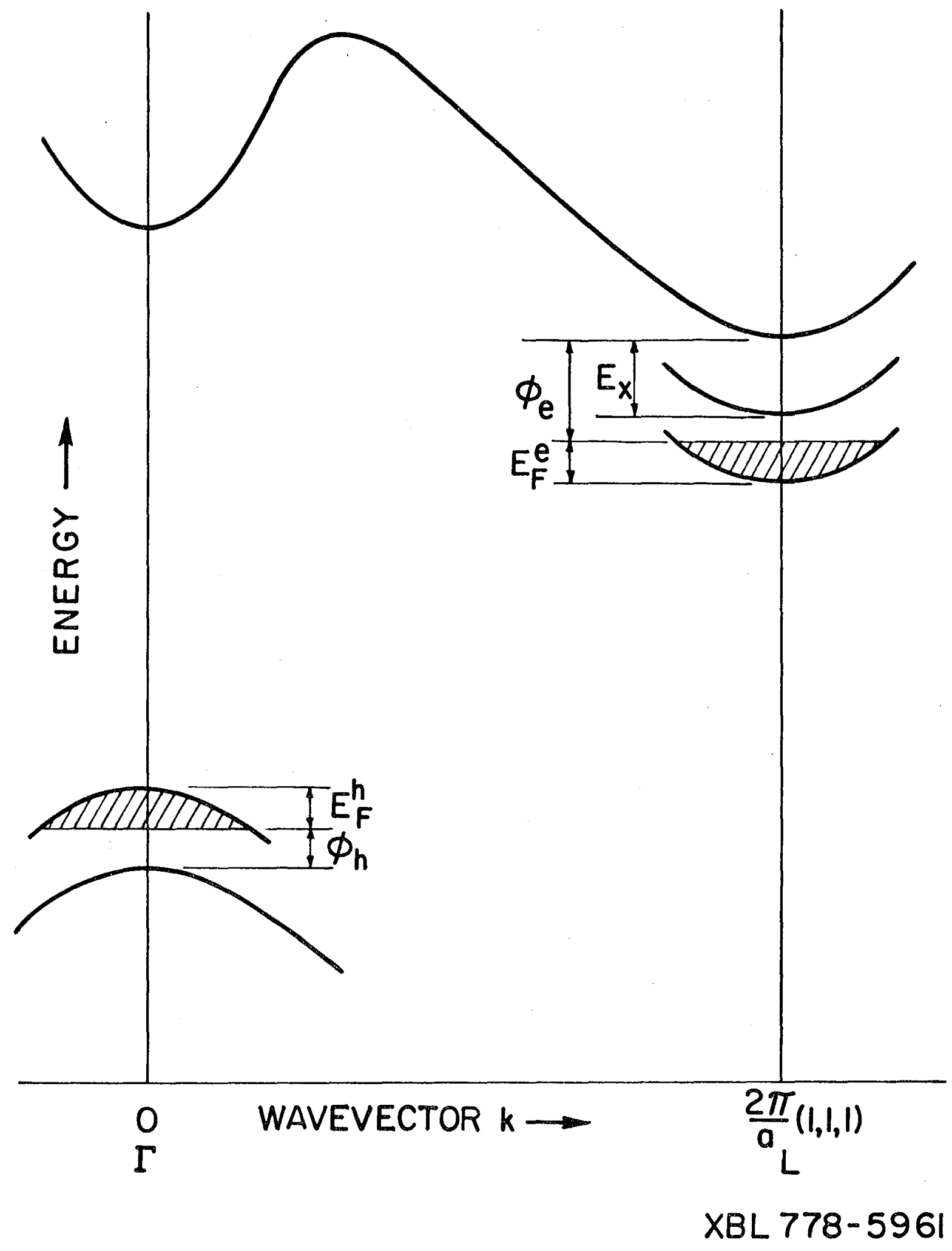

Fig. 1.1 


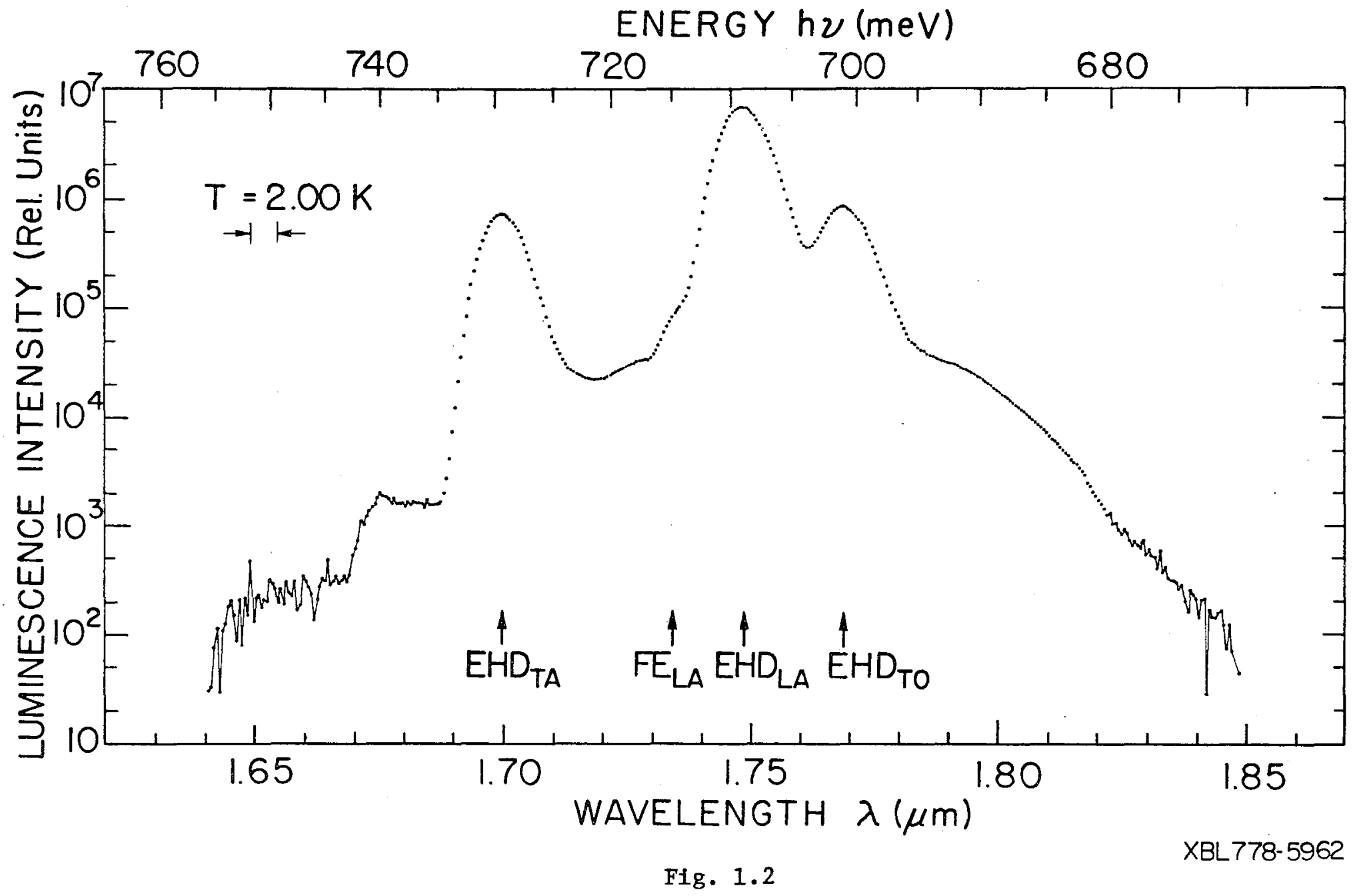

ì 


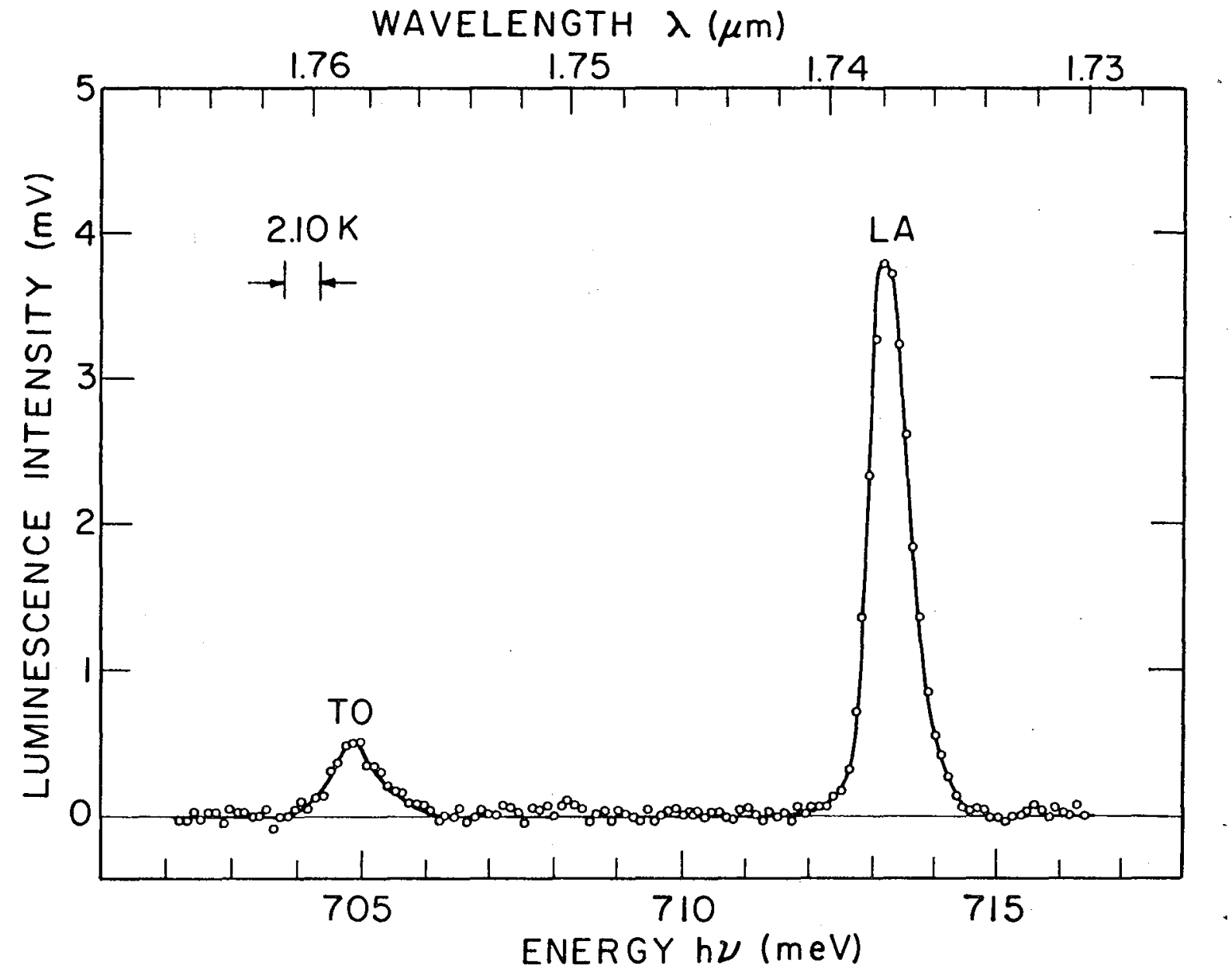

XBL $778-5963$

Fig. 1.3 
$0000+804000$

$-179-$

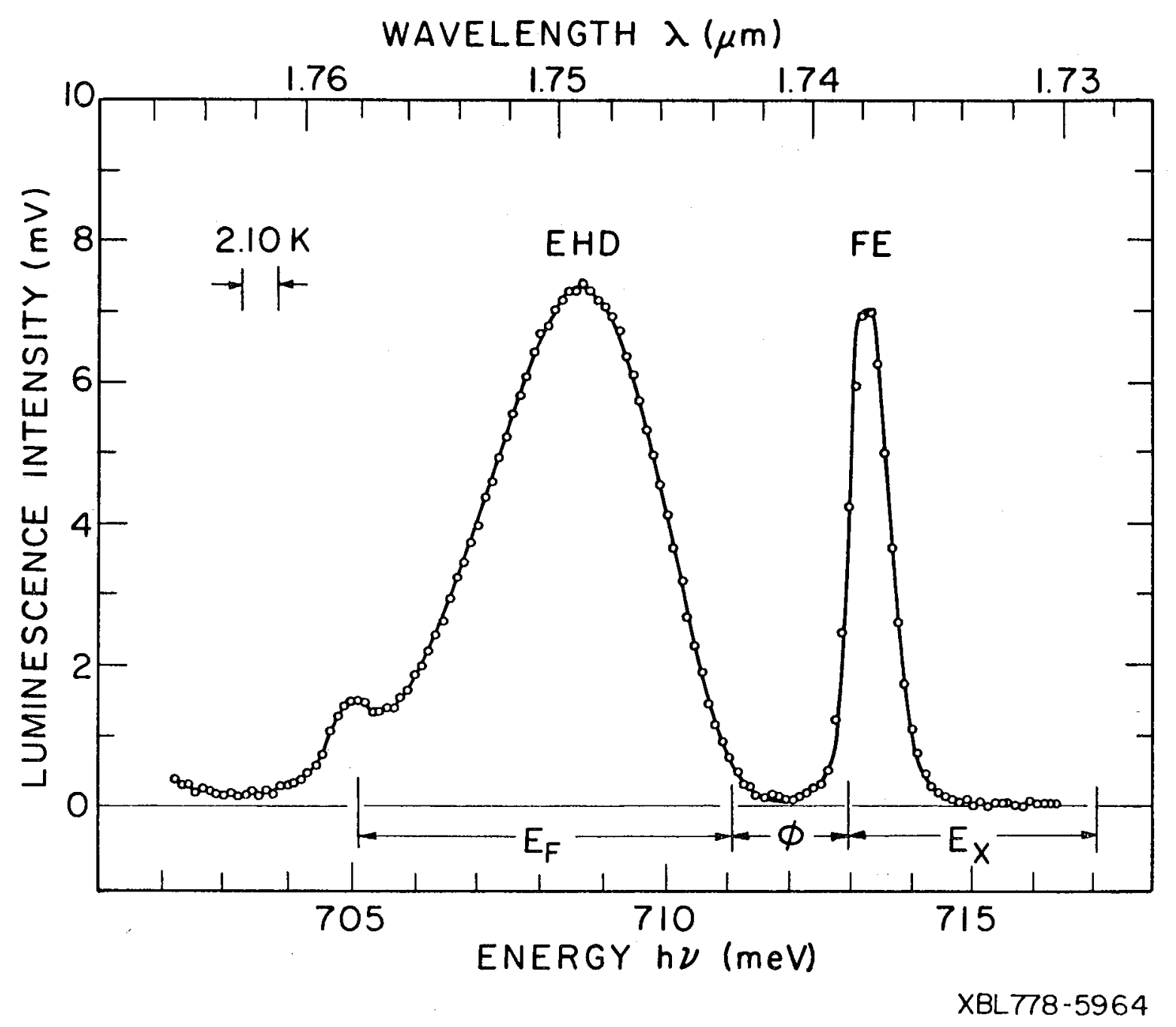

Fig. 1.4 


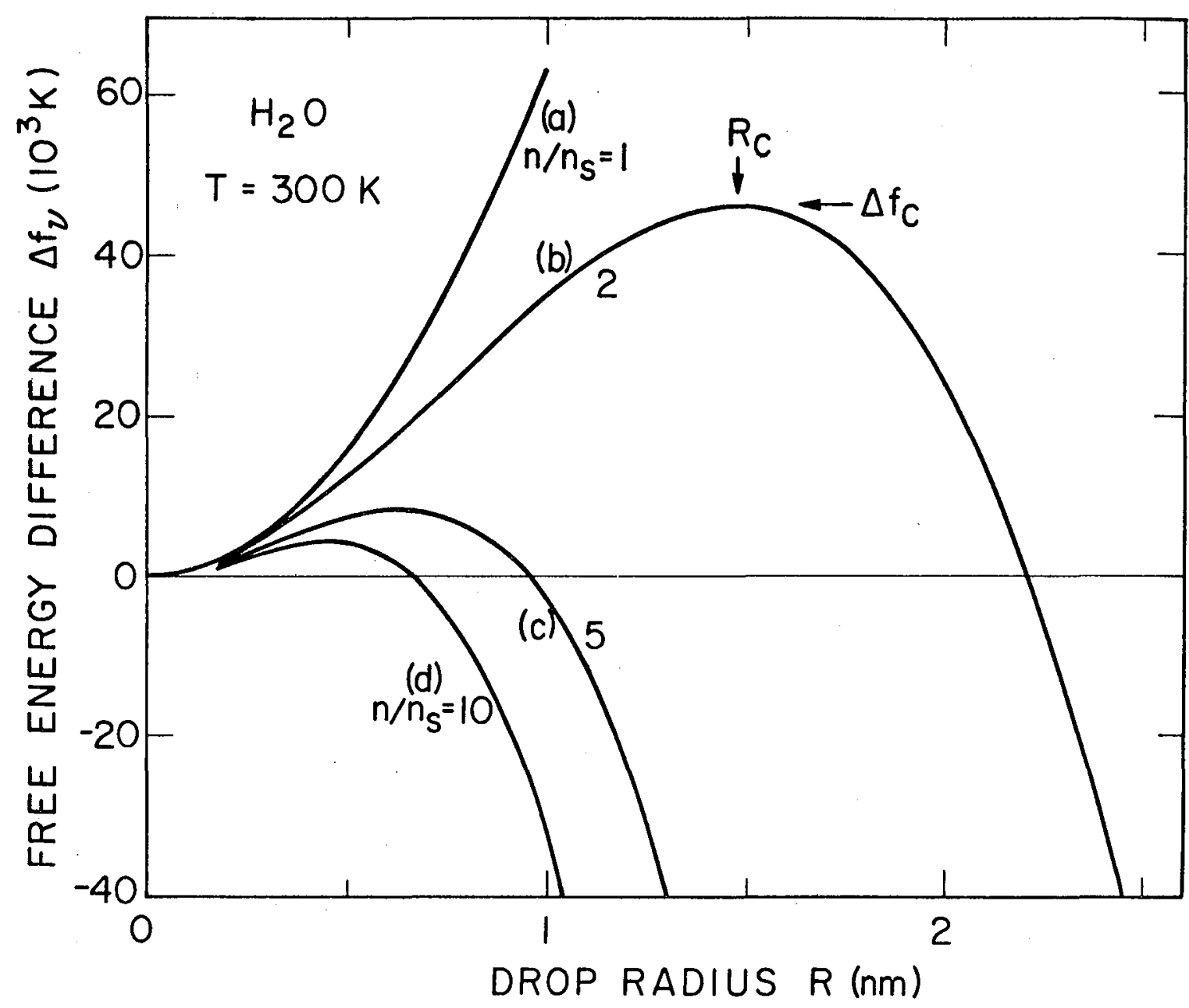

XBL $778-5965$

Fig. 1.5 
00004804069

$-181-$

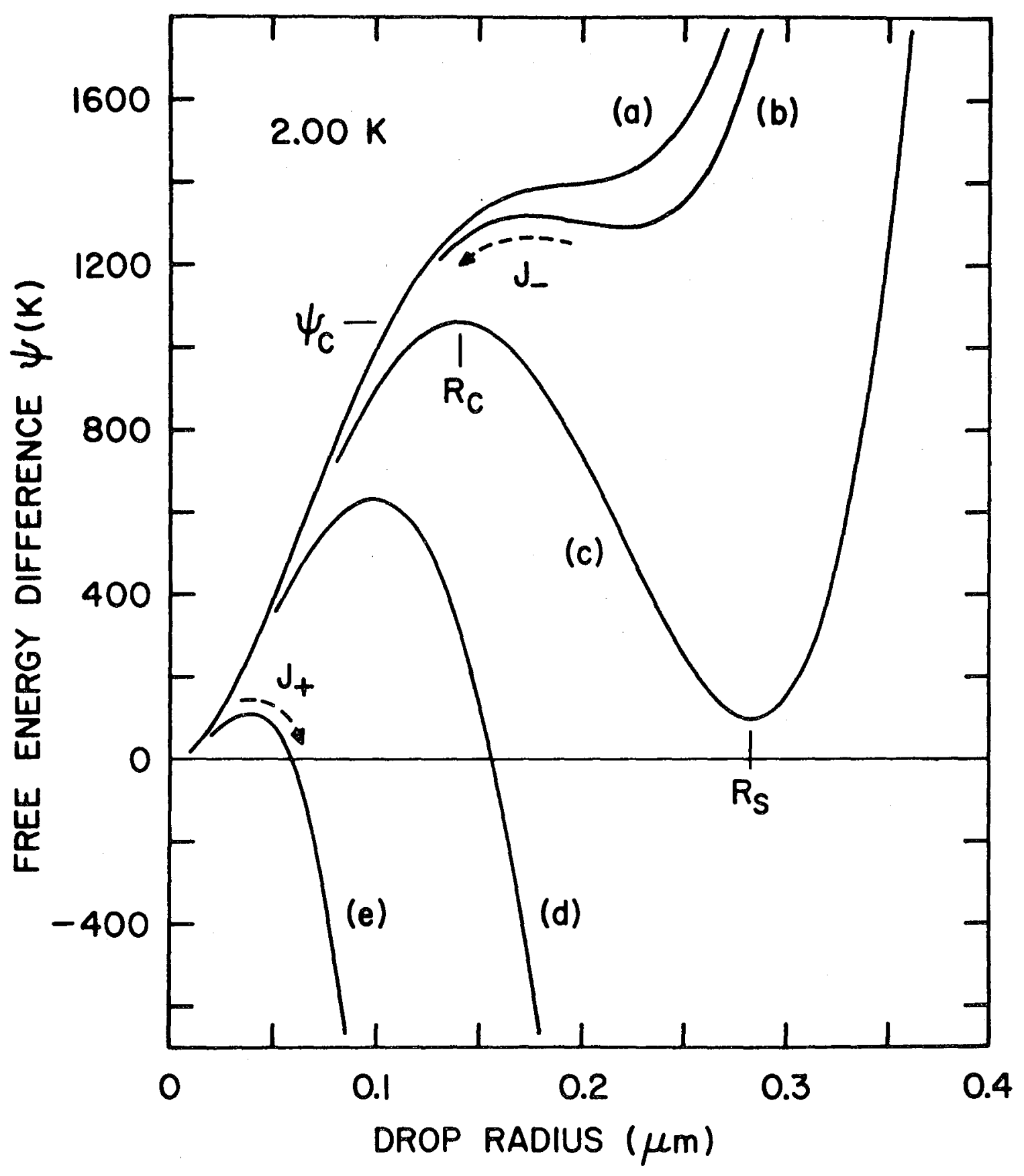

Fig. 2.1

XBL 778-2774 


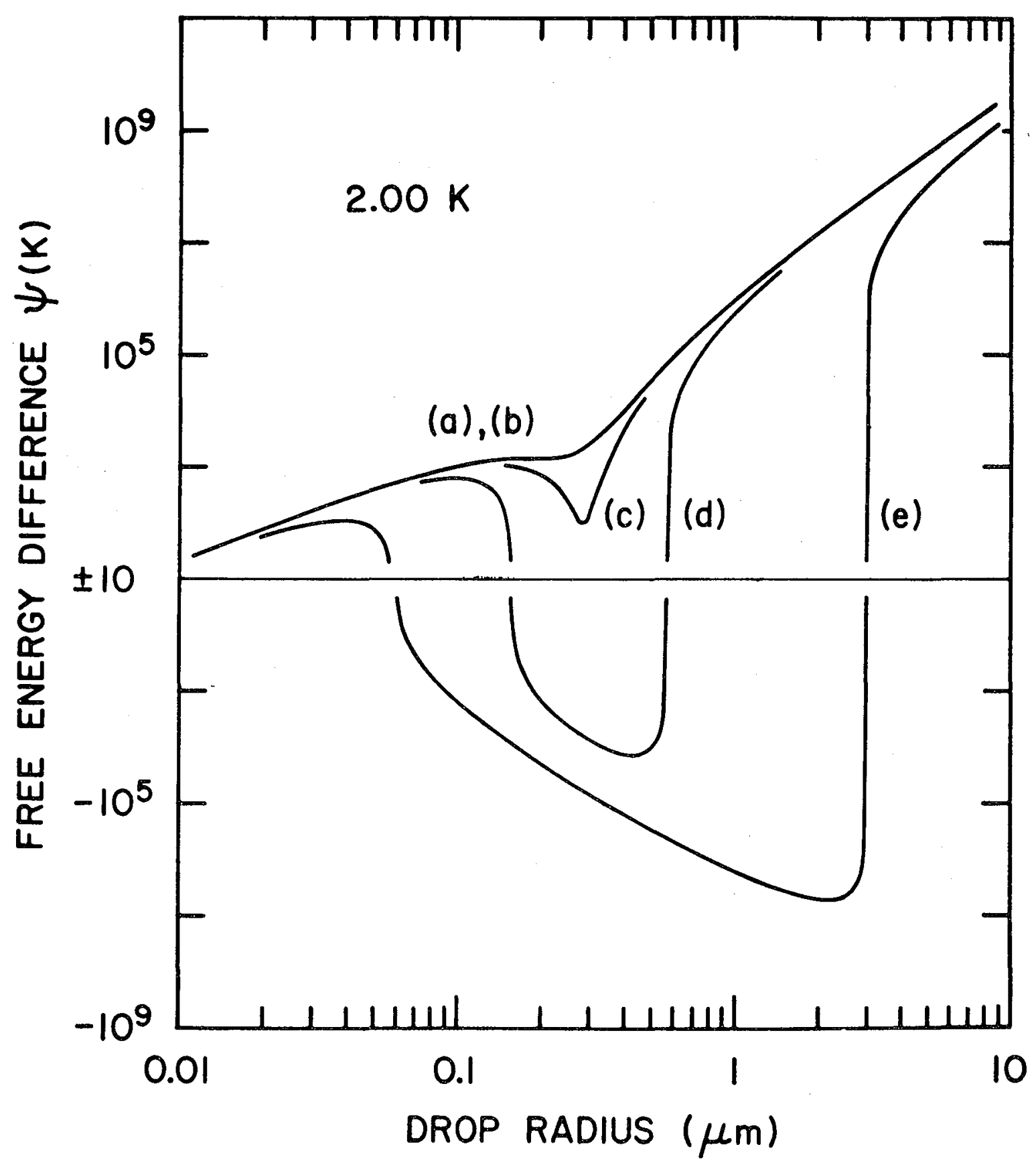

Fig. 2.2 
$00 \times 4004070$

$-183-$
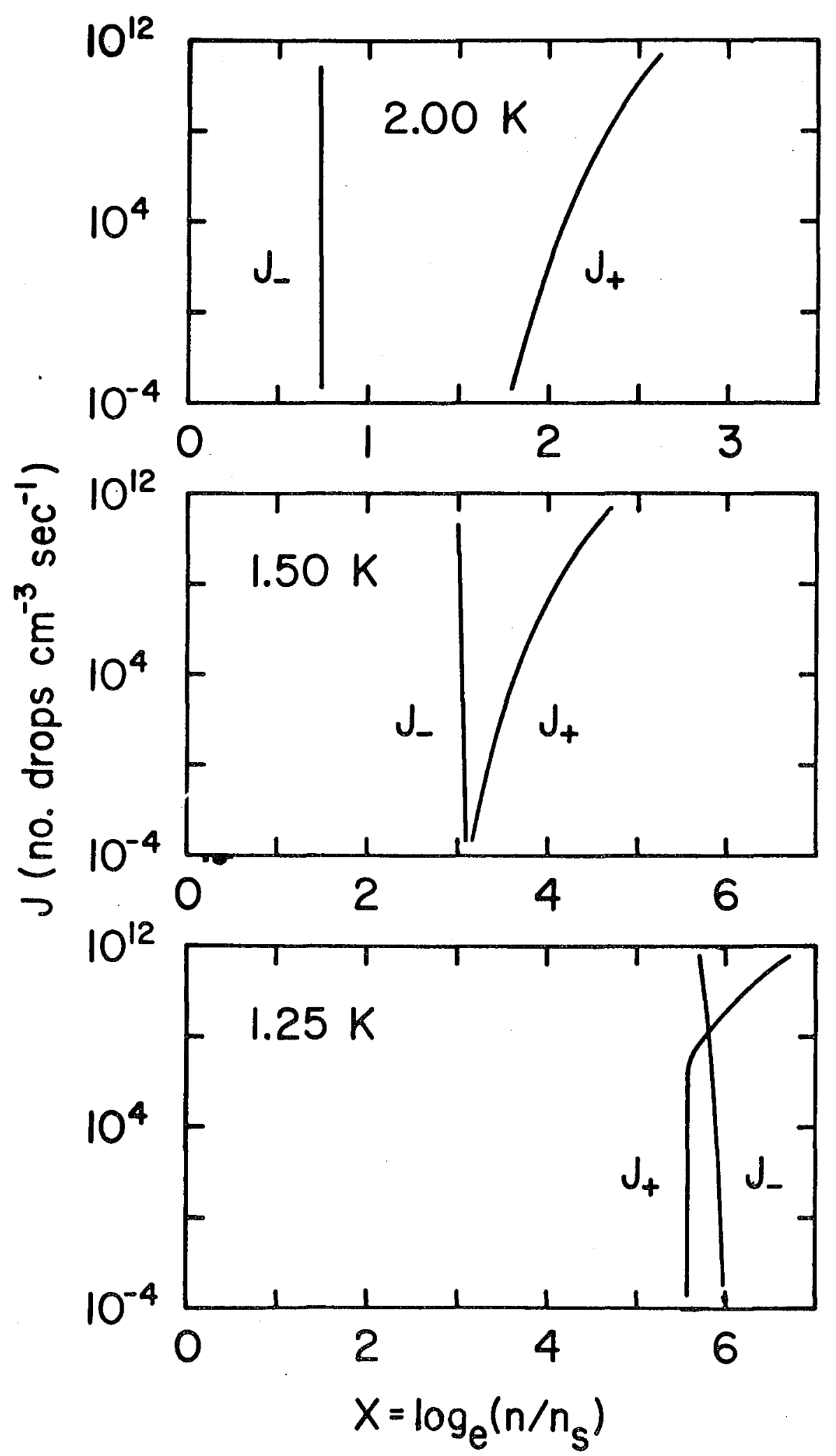

XBL 778-2771

Fig. 2.3 


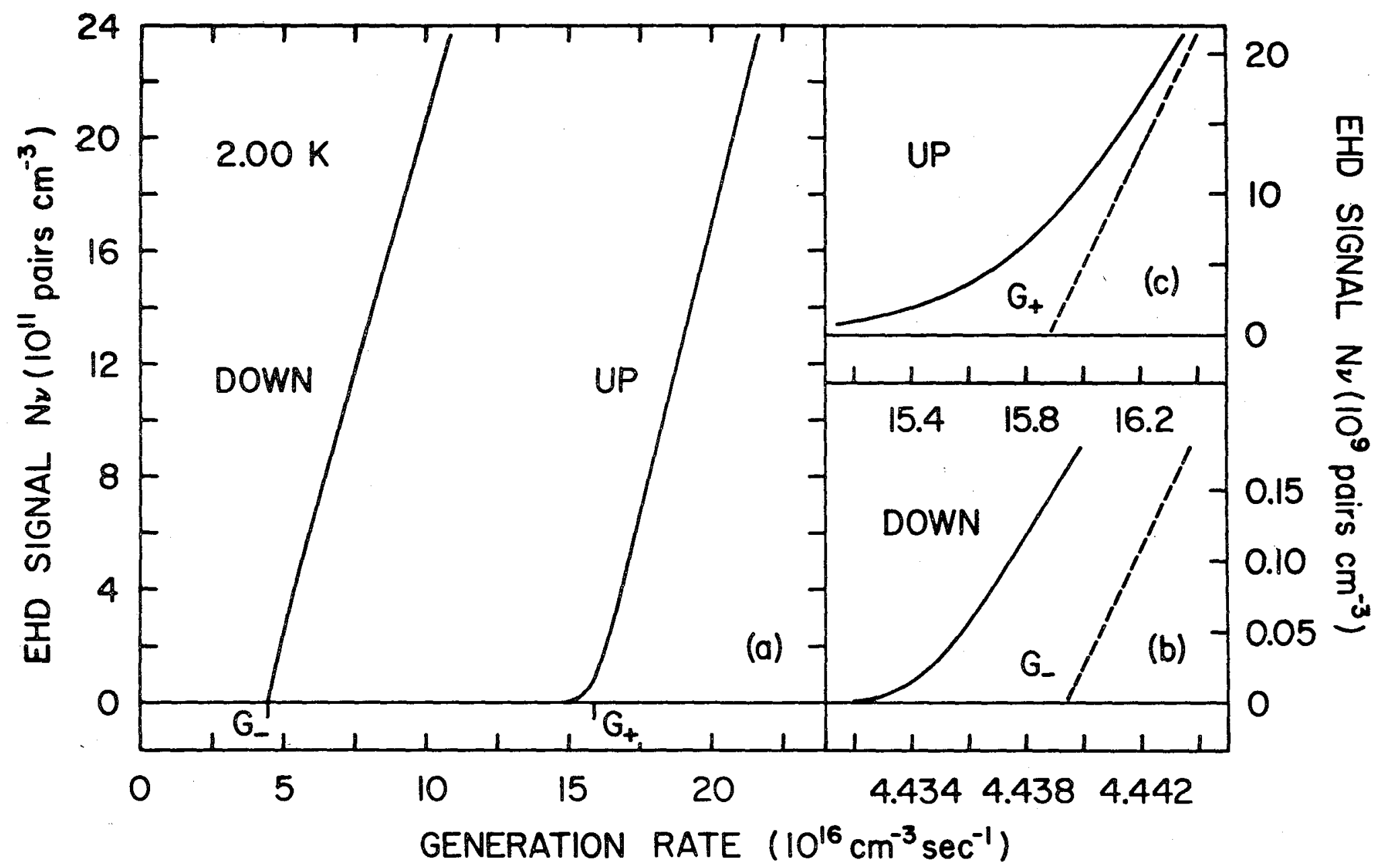

Fig. 3.1 


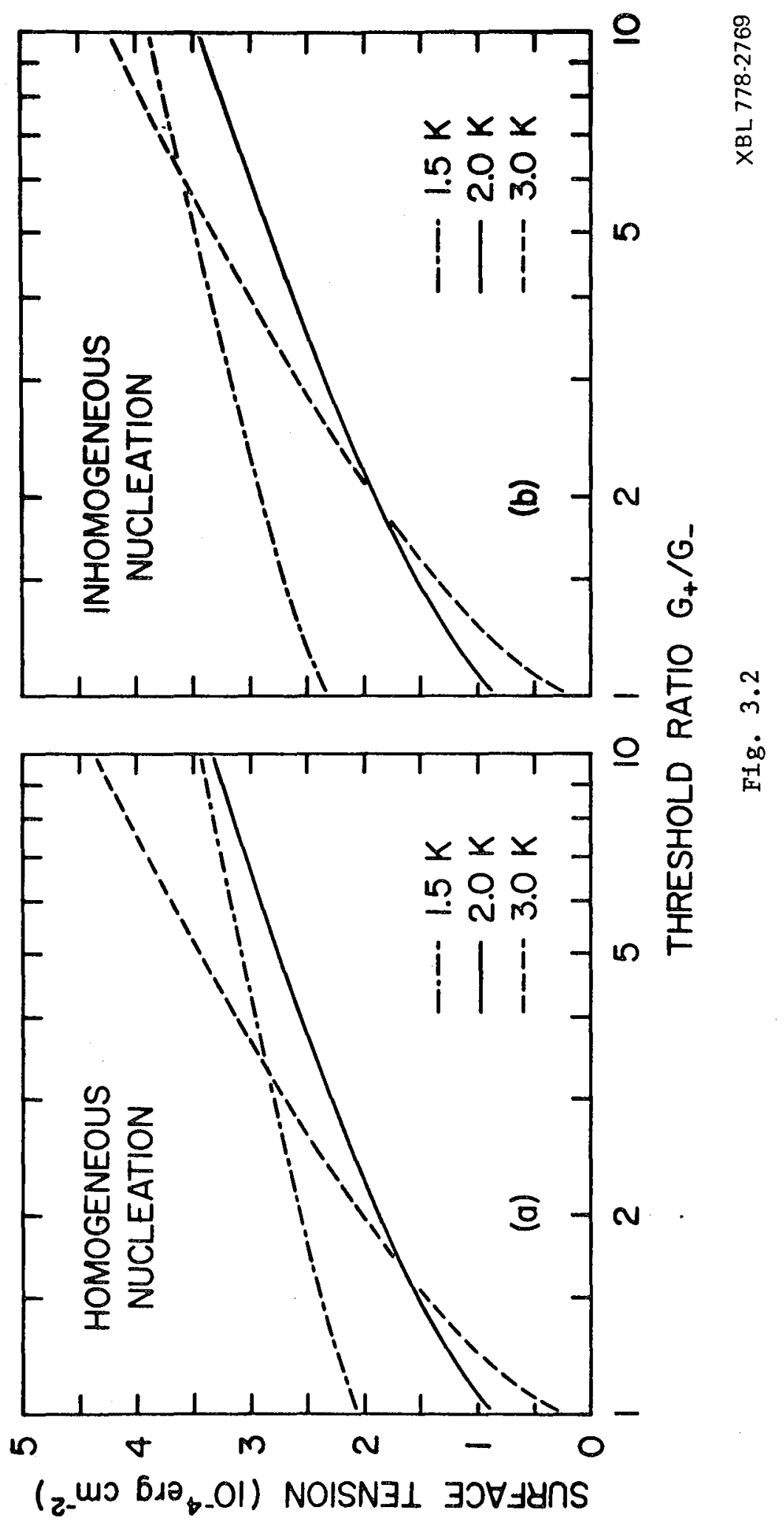




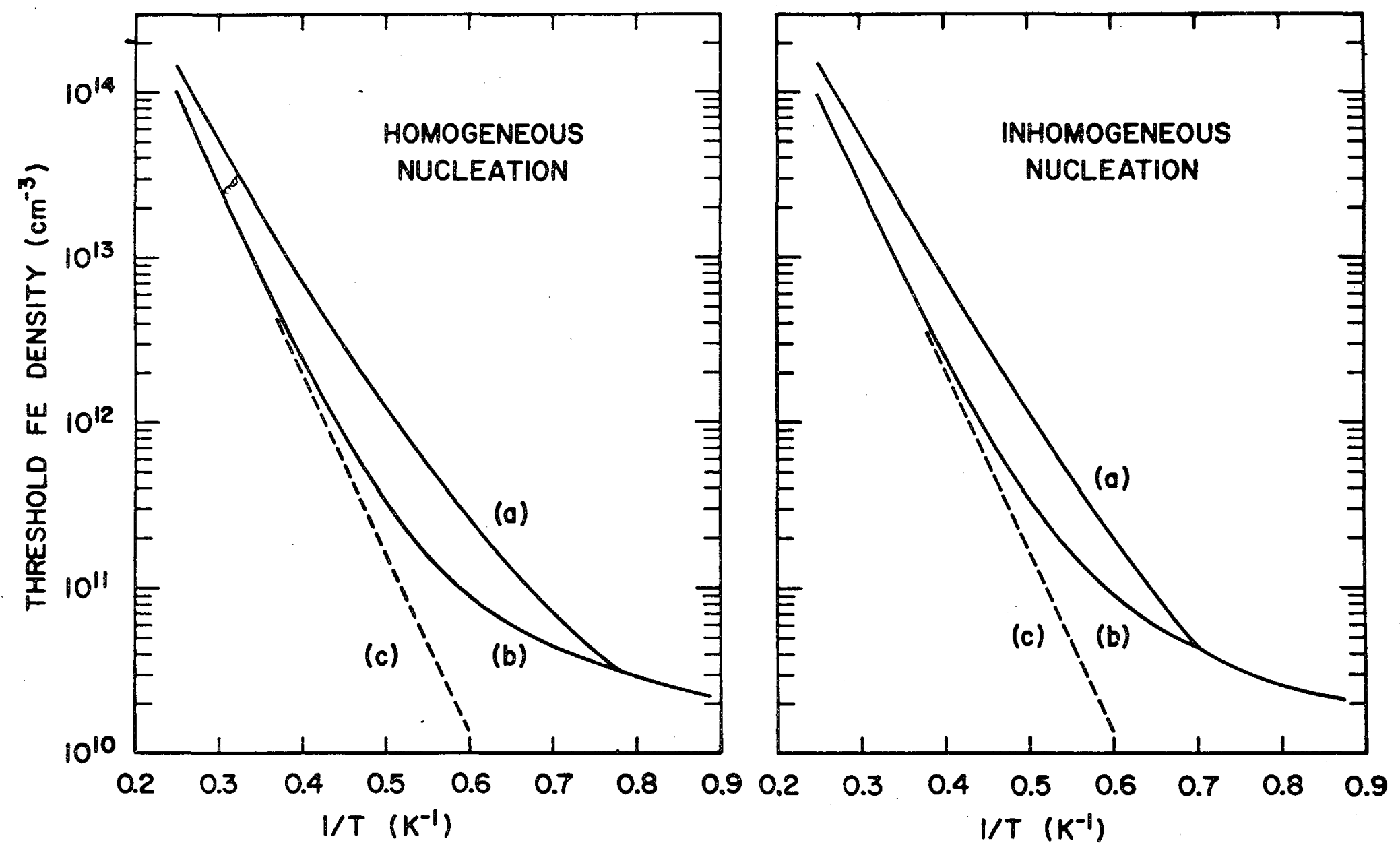


0040404972

$-187-$

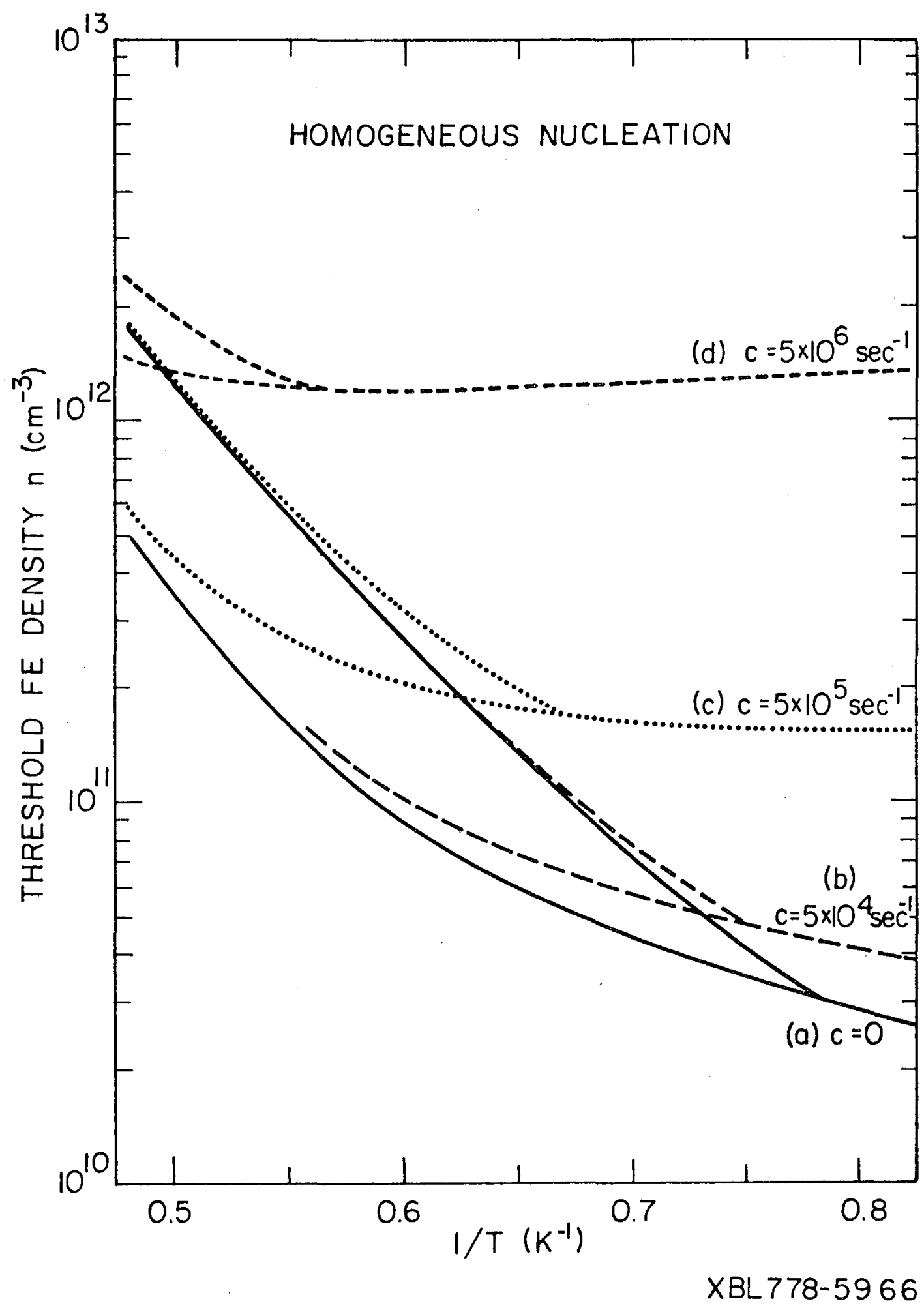

Fig. 3.4 

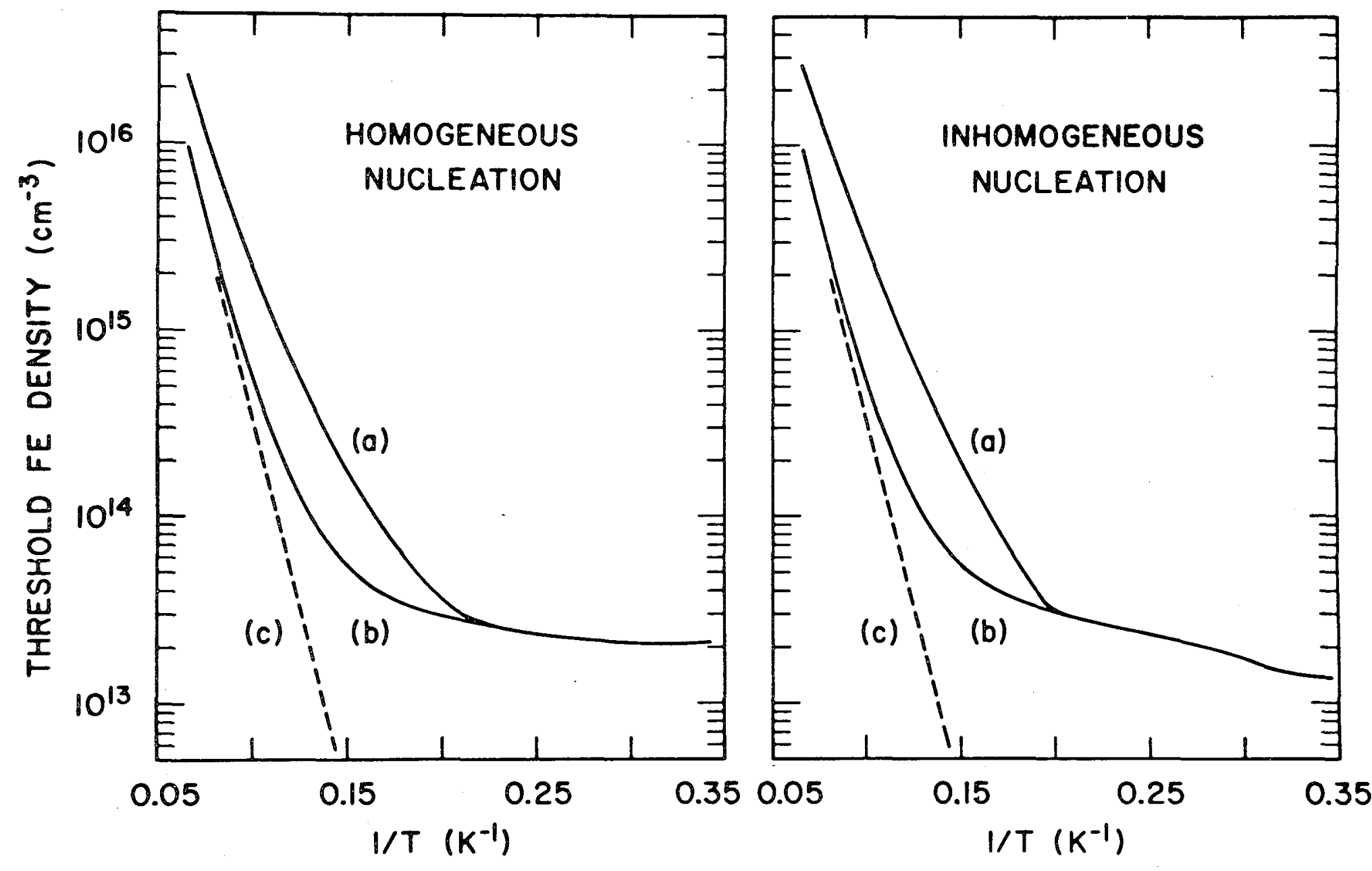

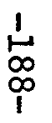

F1g. 3.5 


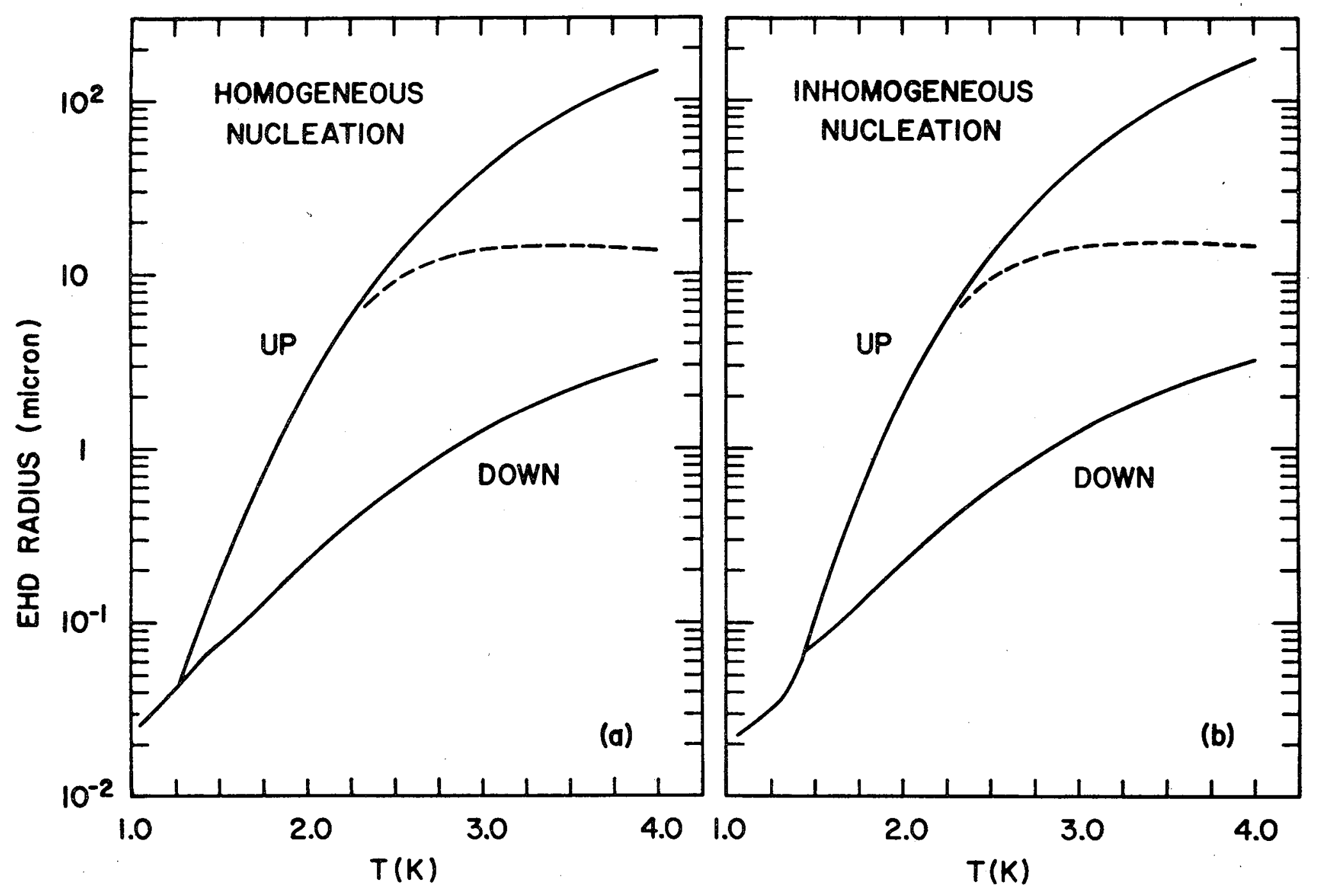

Fig. 3.6 

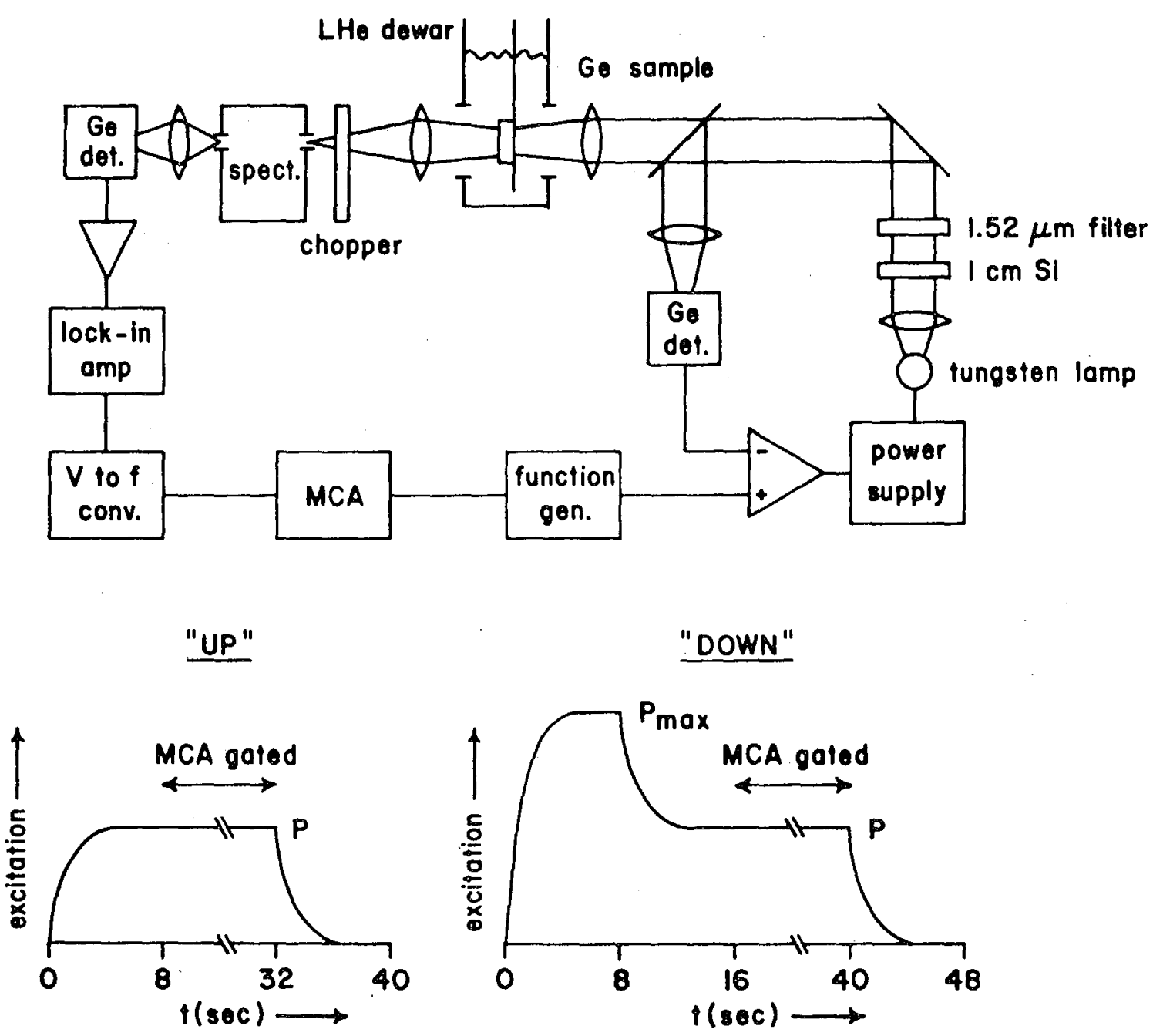

XBL 778-2779

Fig. 4.1 


$$
\begin{aligned}
& 004040449 \\
& -191-
\end{aligned}
$$

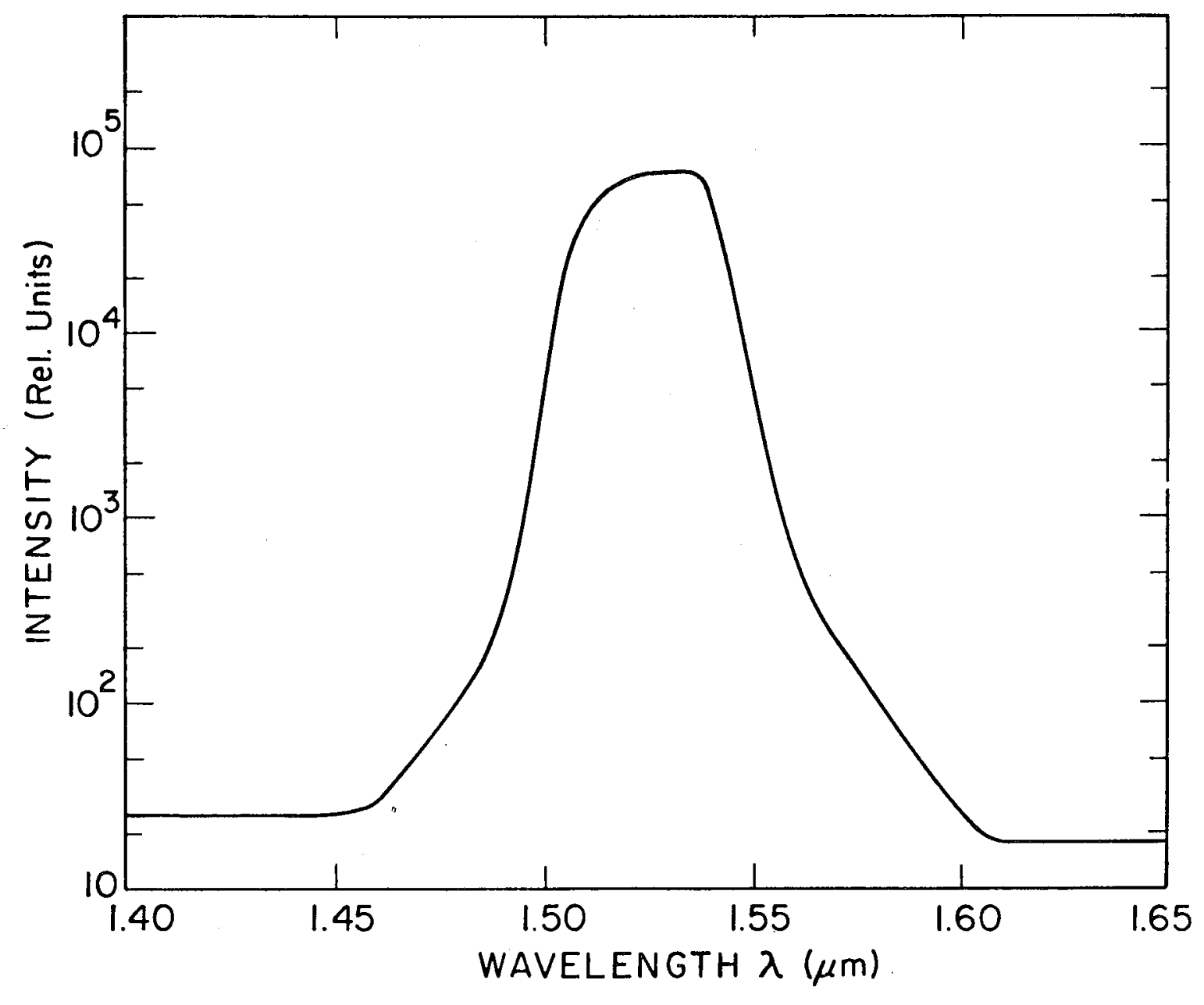

XBL 778-5967

Fig. 4.2 


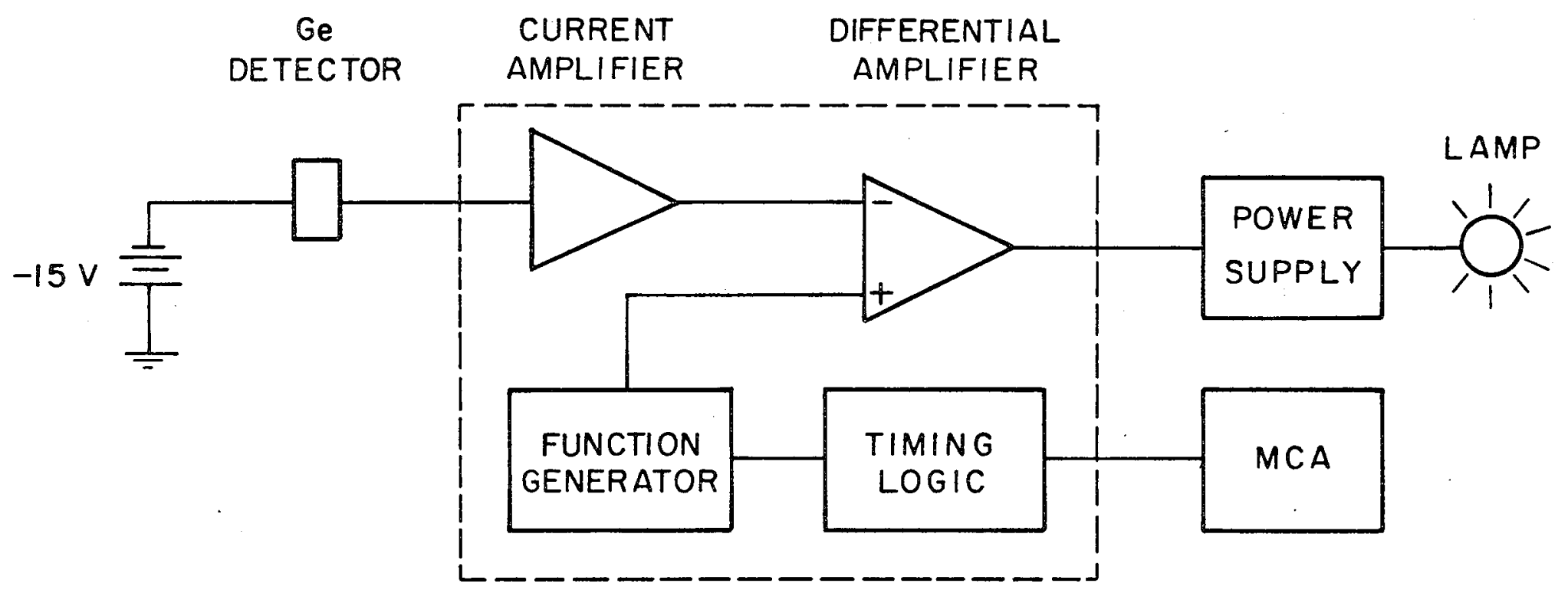

Fig. 4.3

XBL 778-5968 
(a) REFERENCE FUNCTION GENERATOR

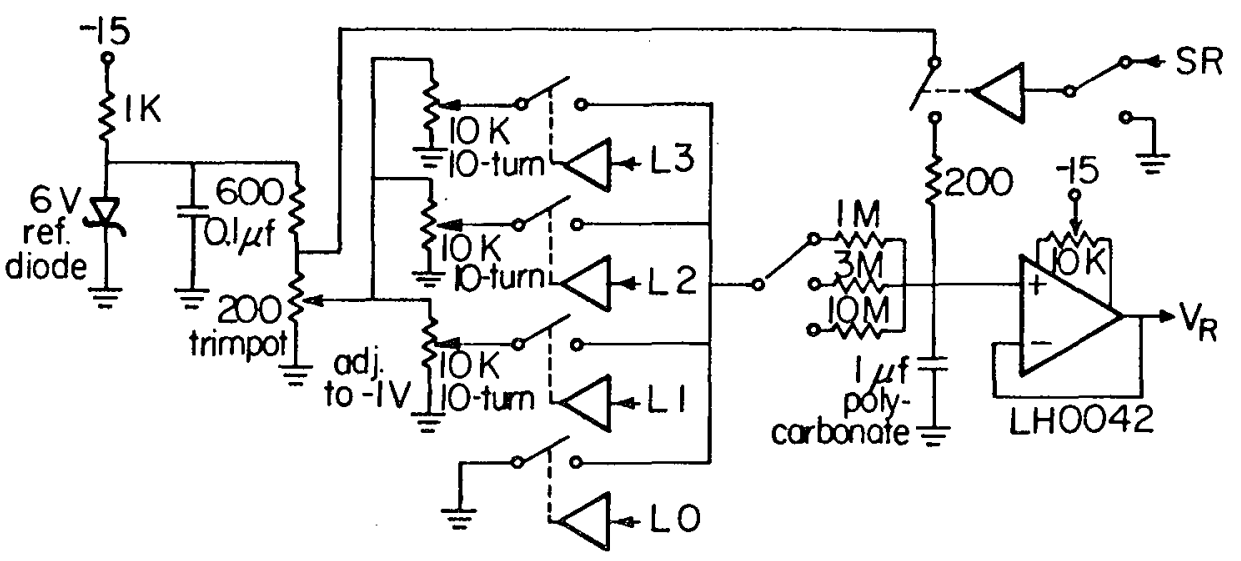

(b)

CURRENT AMPLIFIER

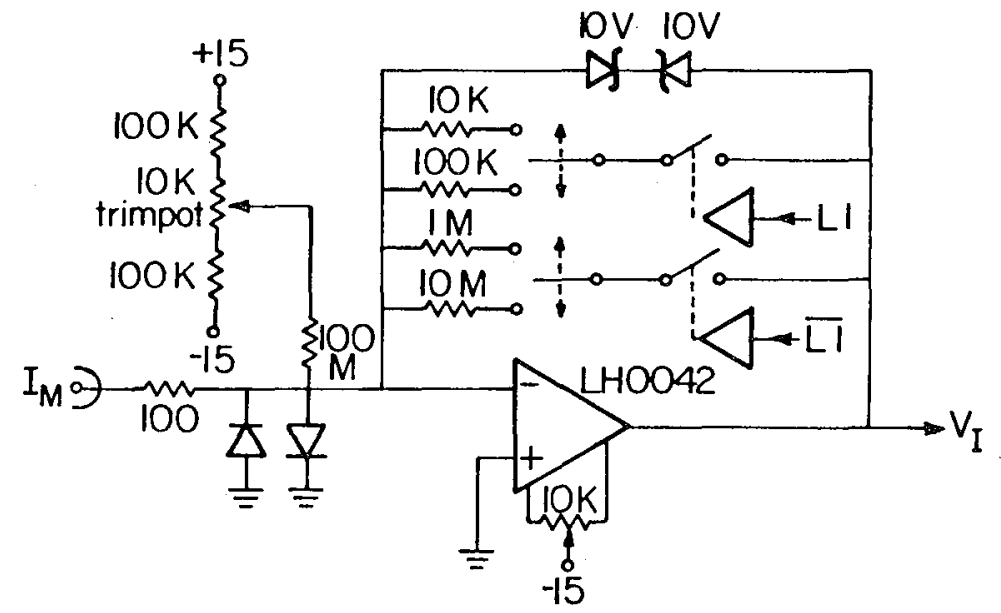

(c)

DIFFERENTIAL AMPLIFIER

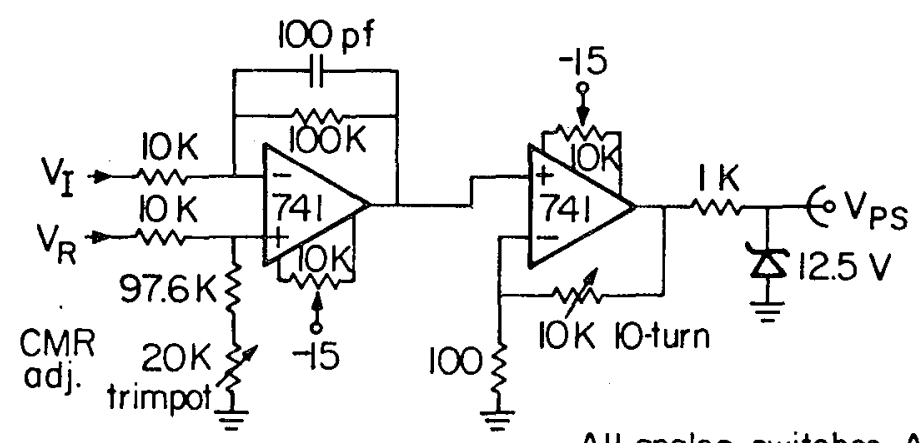

All analog switches $A D 2010$

All resistors $1 \%$ metal film

F1g. 4.4 
TIMING LOGIC

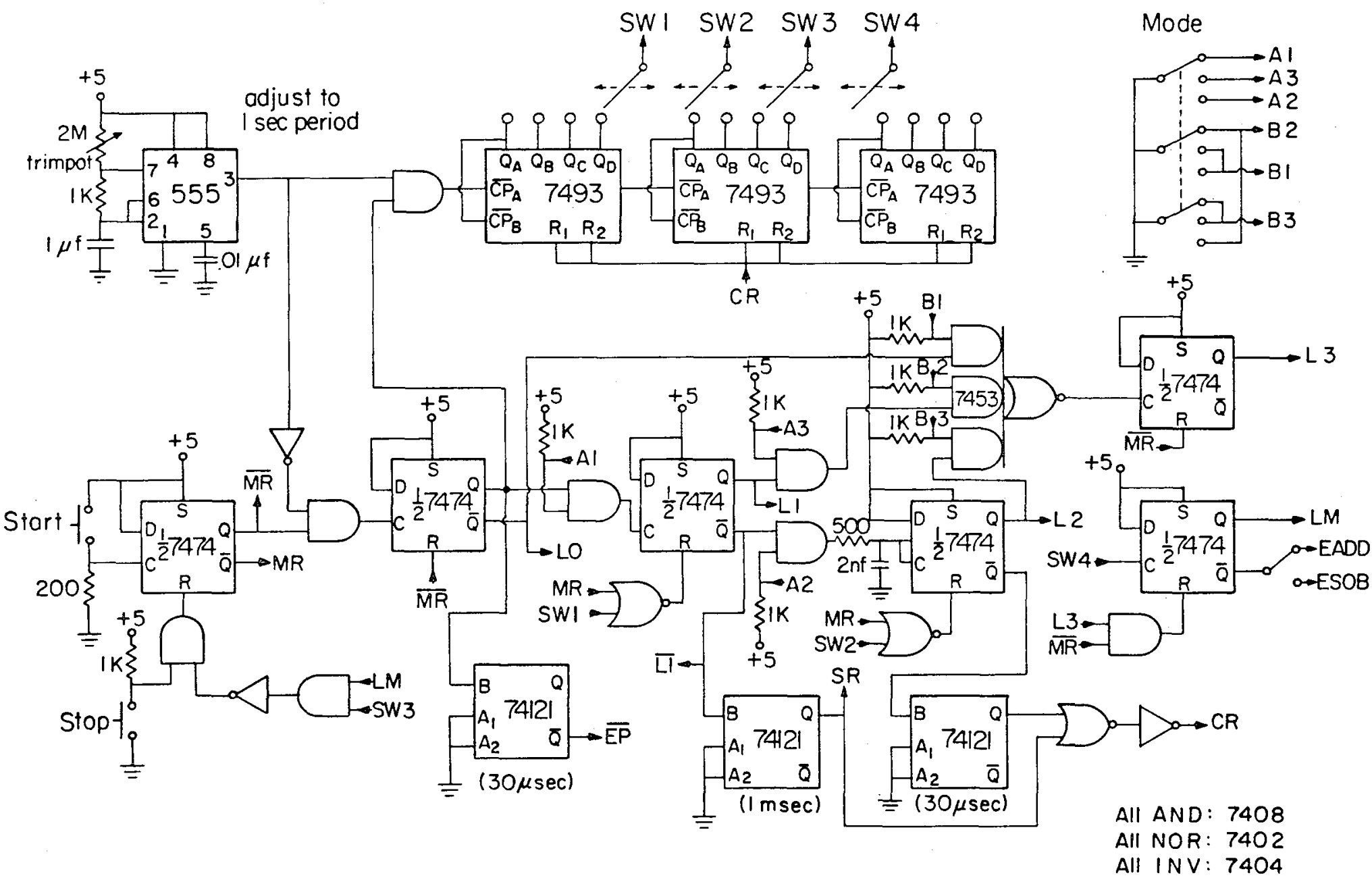



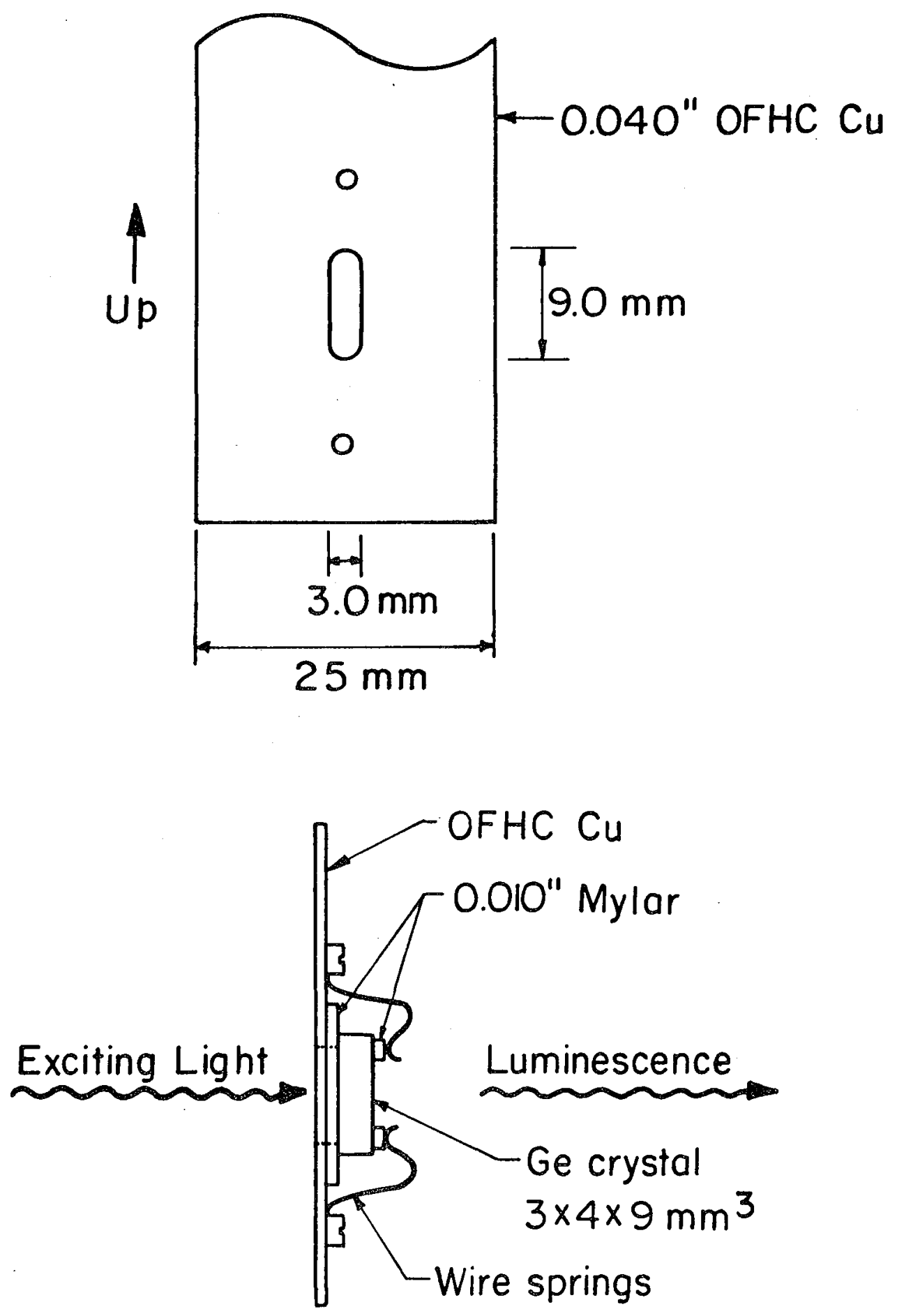

XBL778-597I

Fig. 4.6 


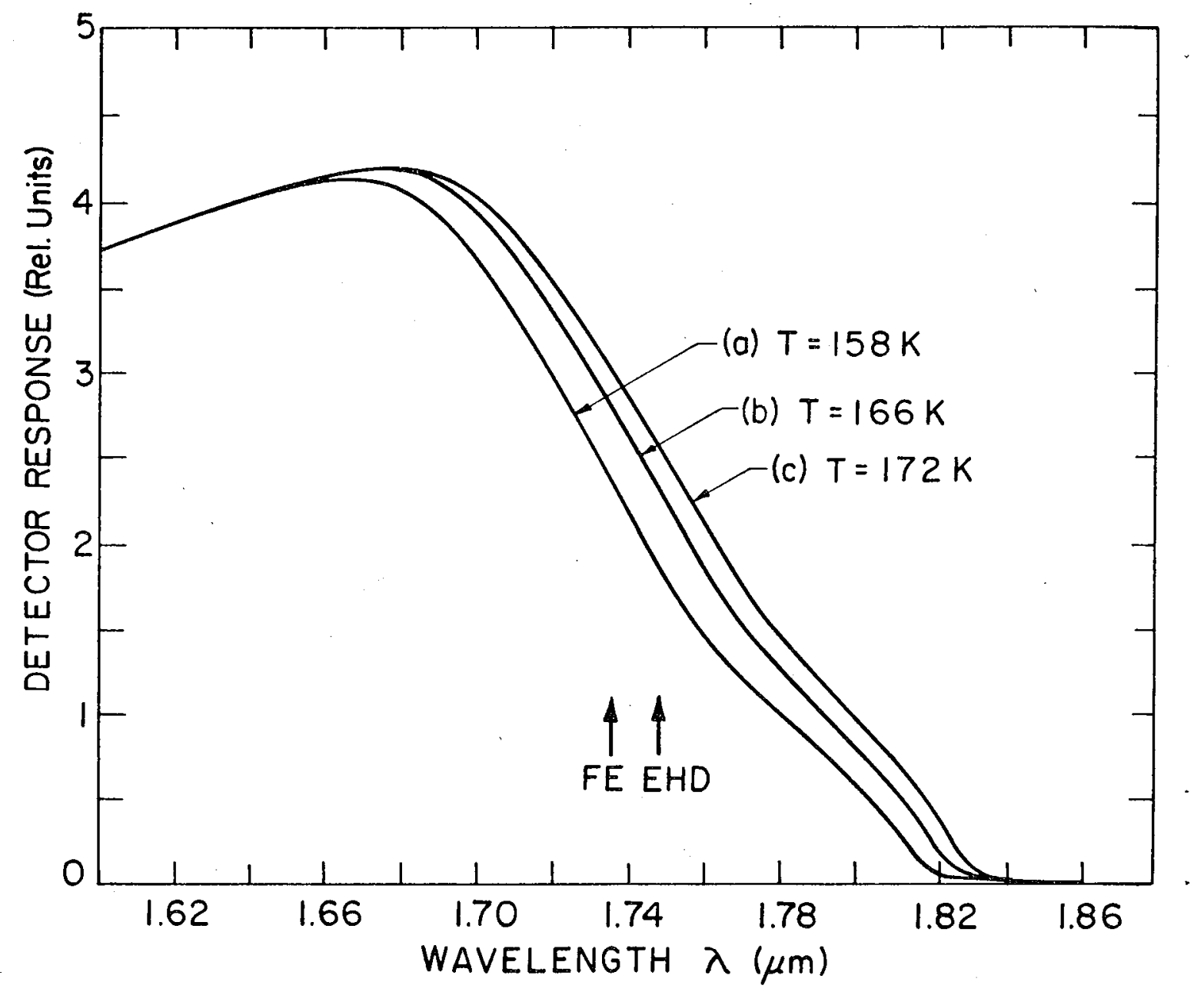

XBL 778-5972

Fig. 4.7 
SENSITIVE CURRENT AMPLIFIER AND DETECTOR BIASING CIRCUIT
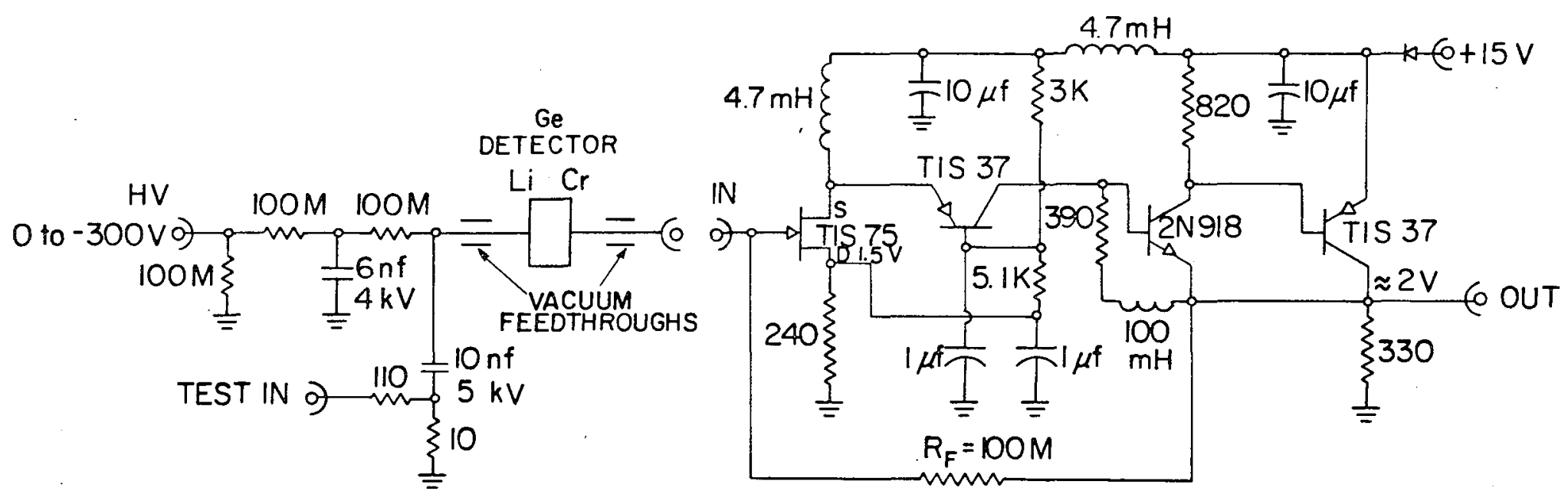

F1g. 4.8

$X B L 778-5973$ 
SELECTIVE LOW-LEVEL CLIPPER

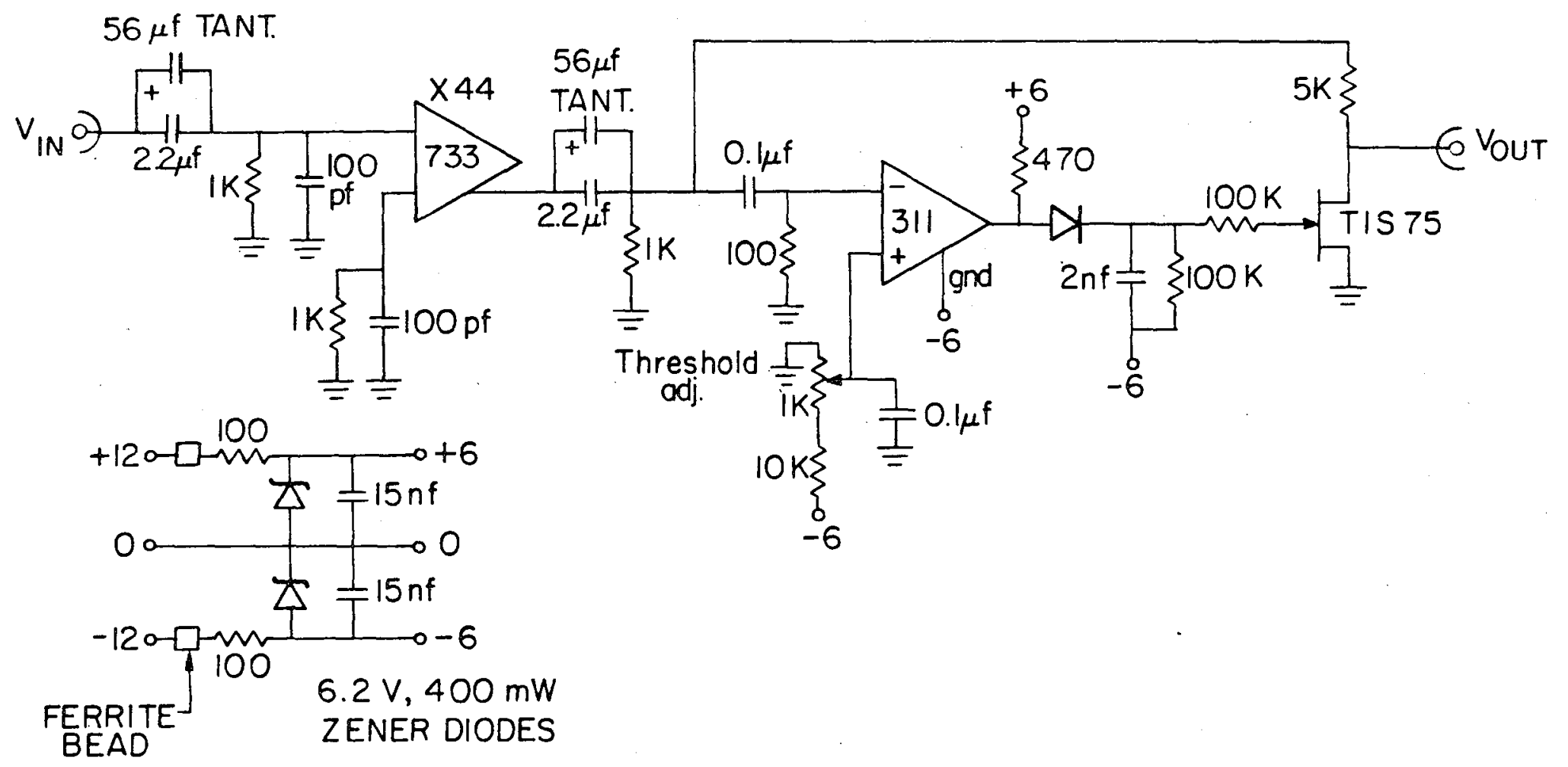


$00+4304978$

-199-

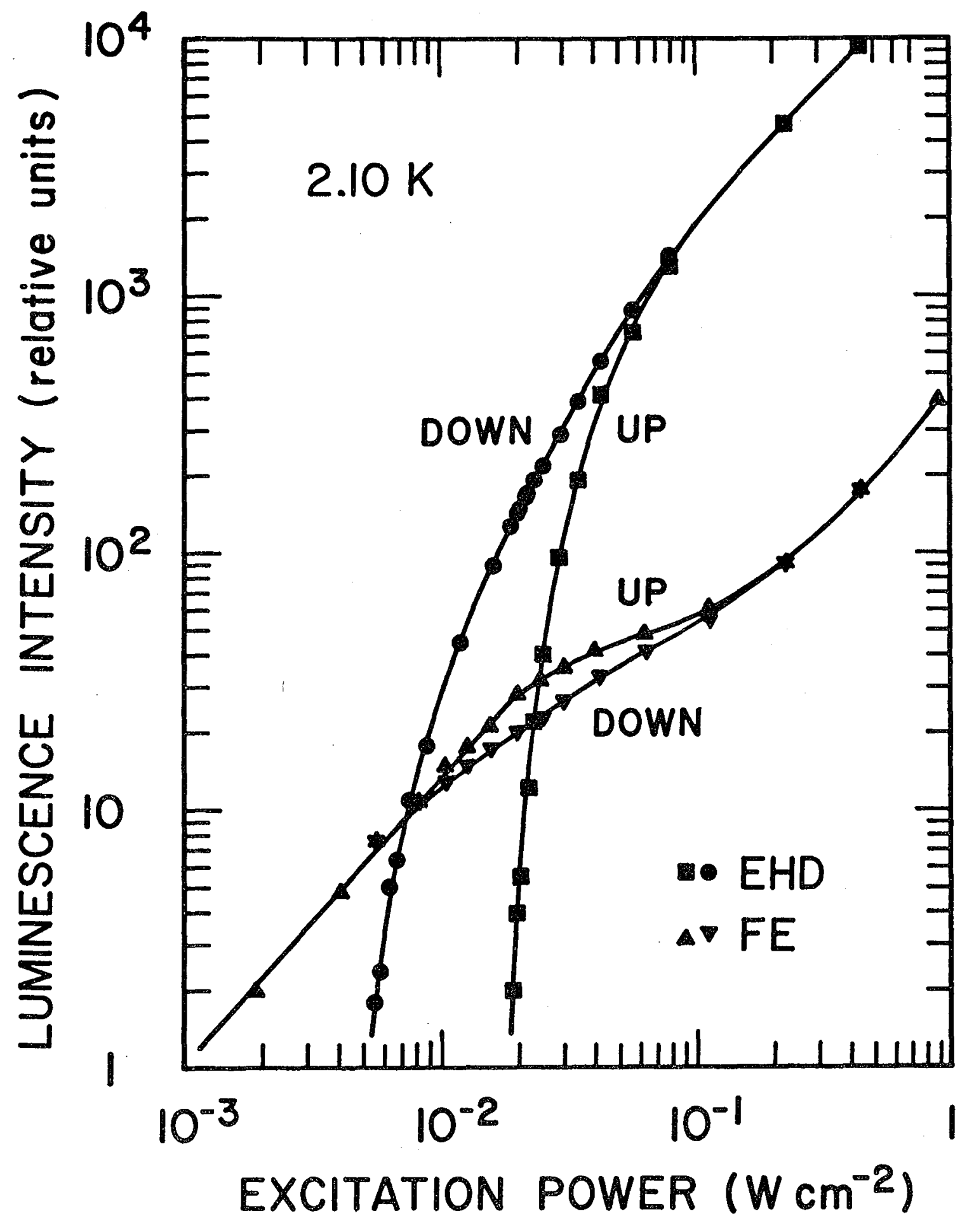

XBL 778-2751

F1g. 5.1 


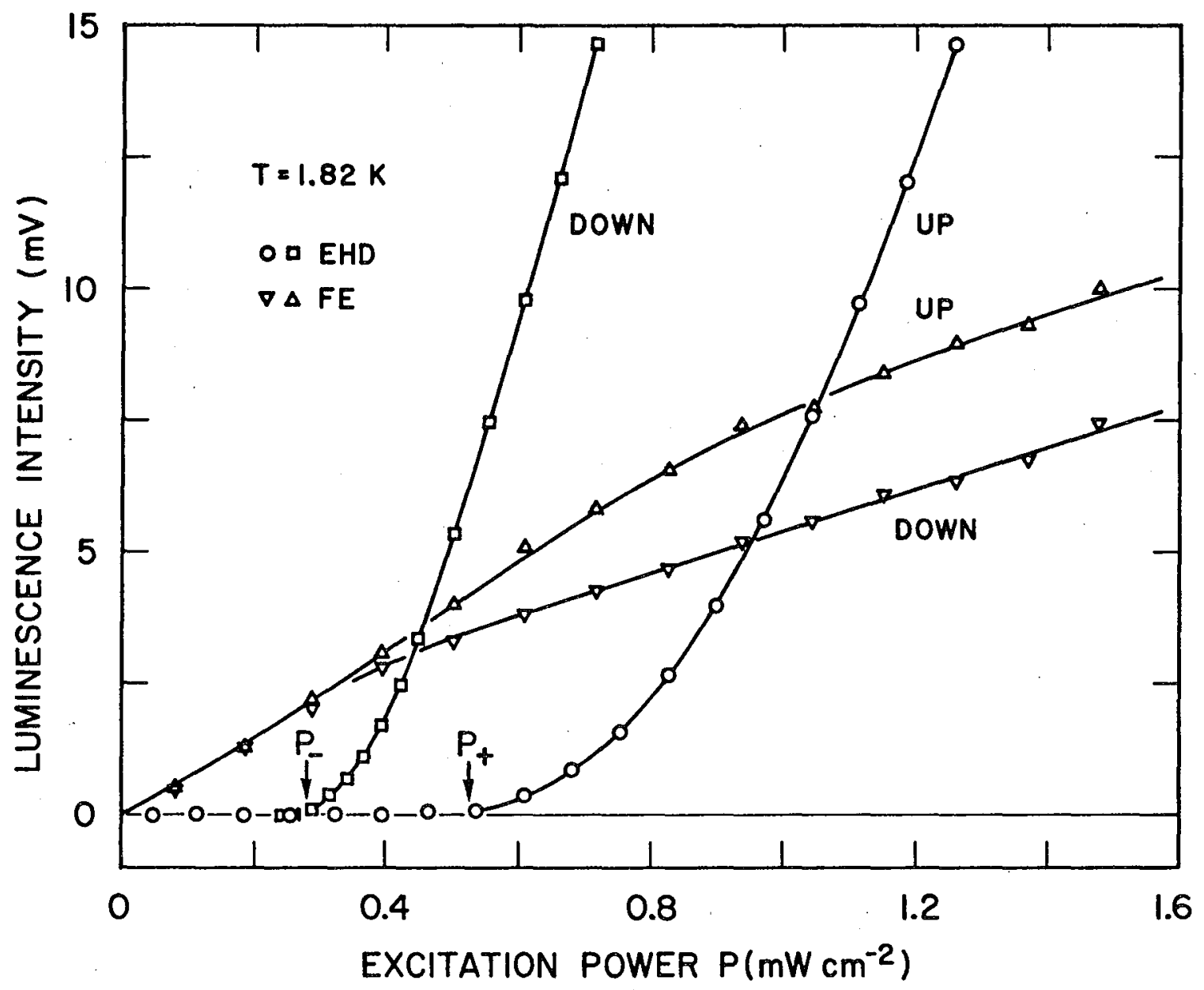

XBL 778-5975

F1g. 5.2 
$00+4004379$

$-201-$

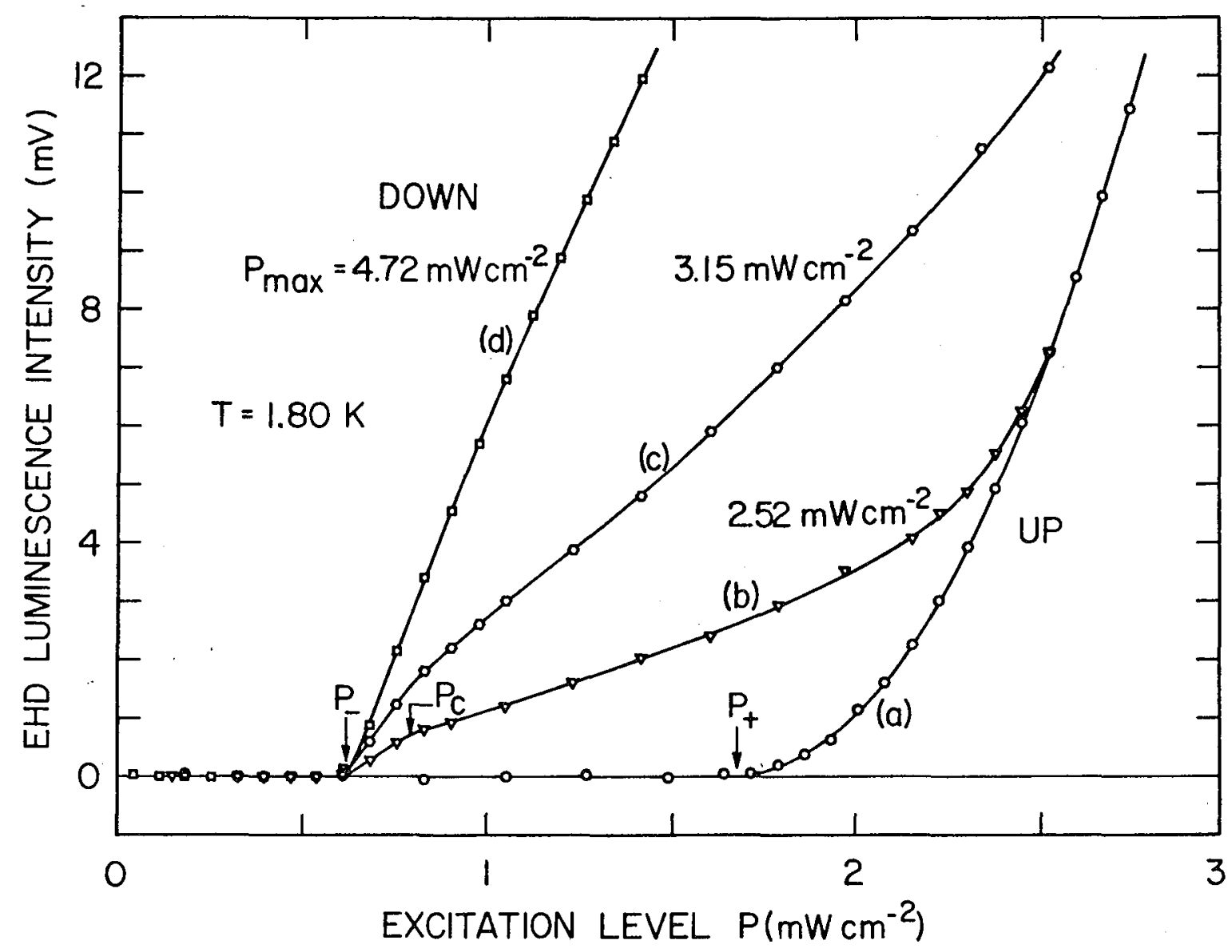

Fig. 5.3

XBL 778-5976 


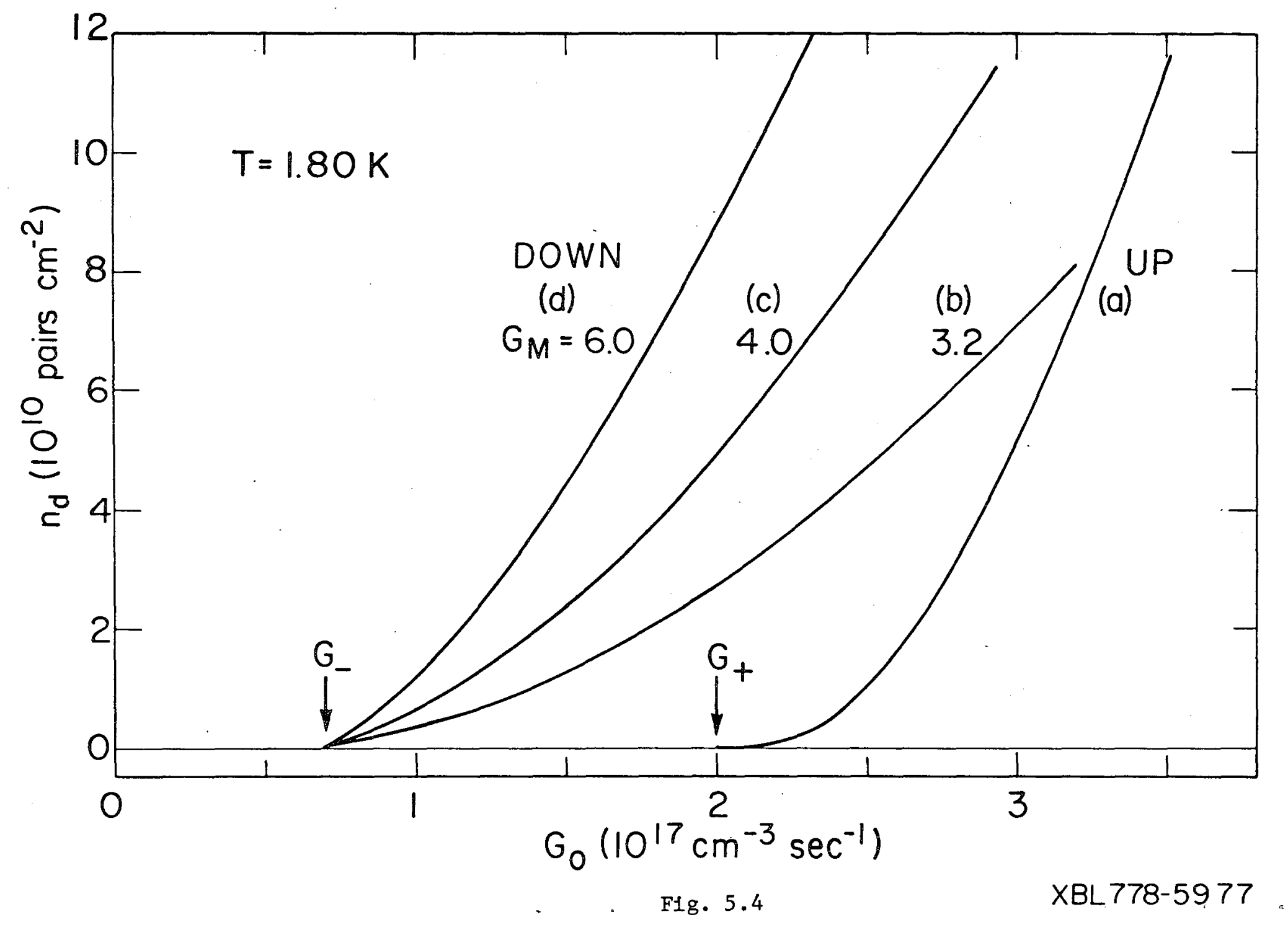

N 


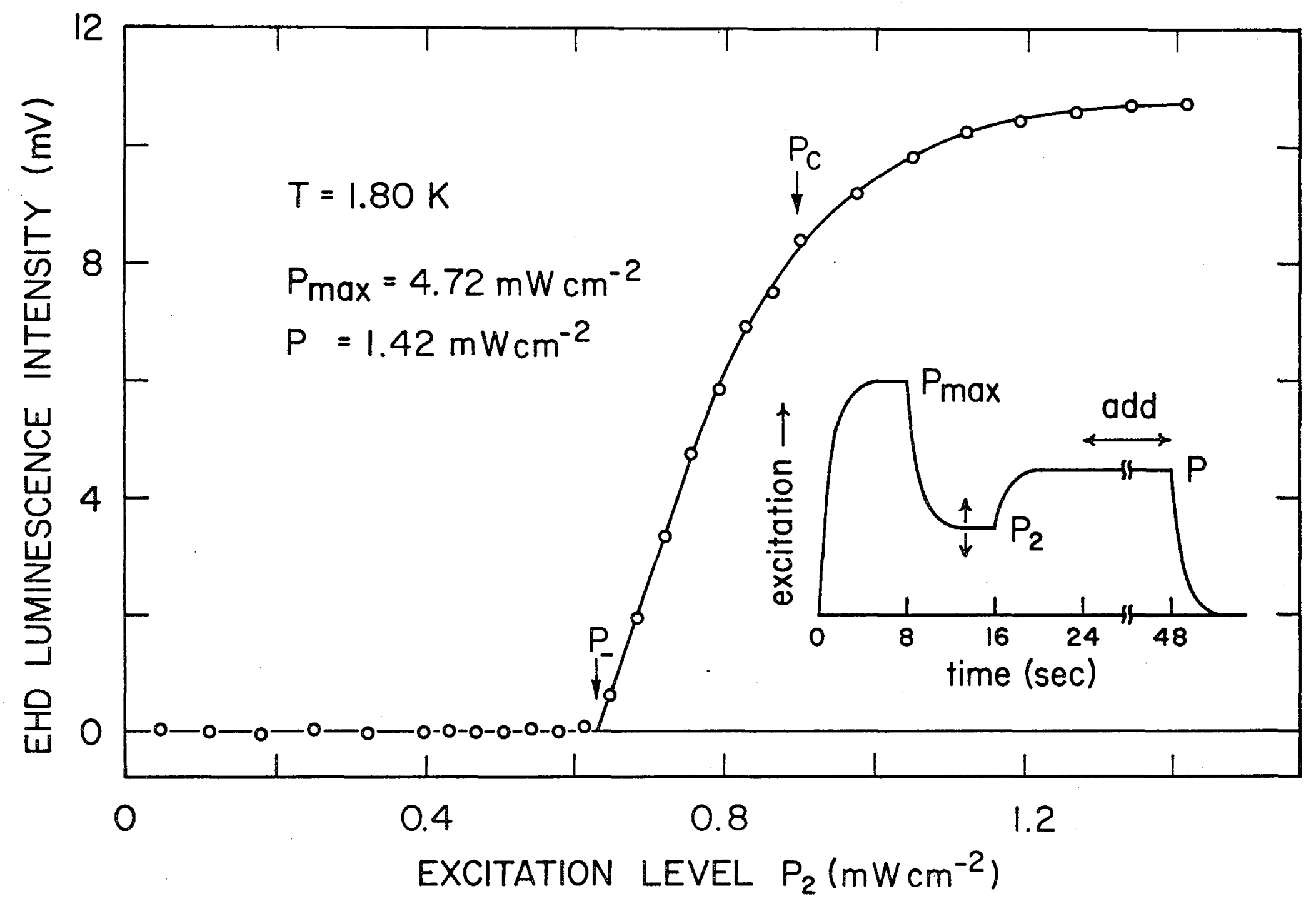

F1g. 5.5 


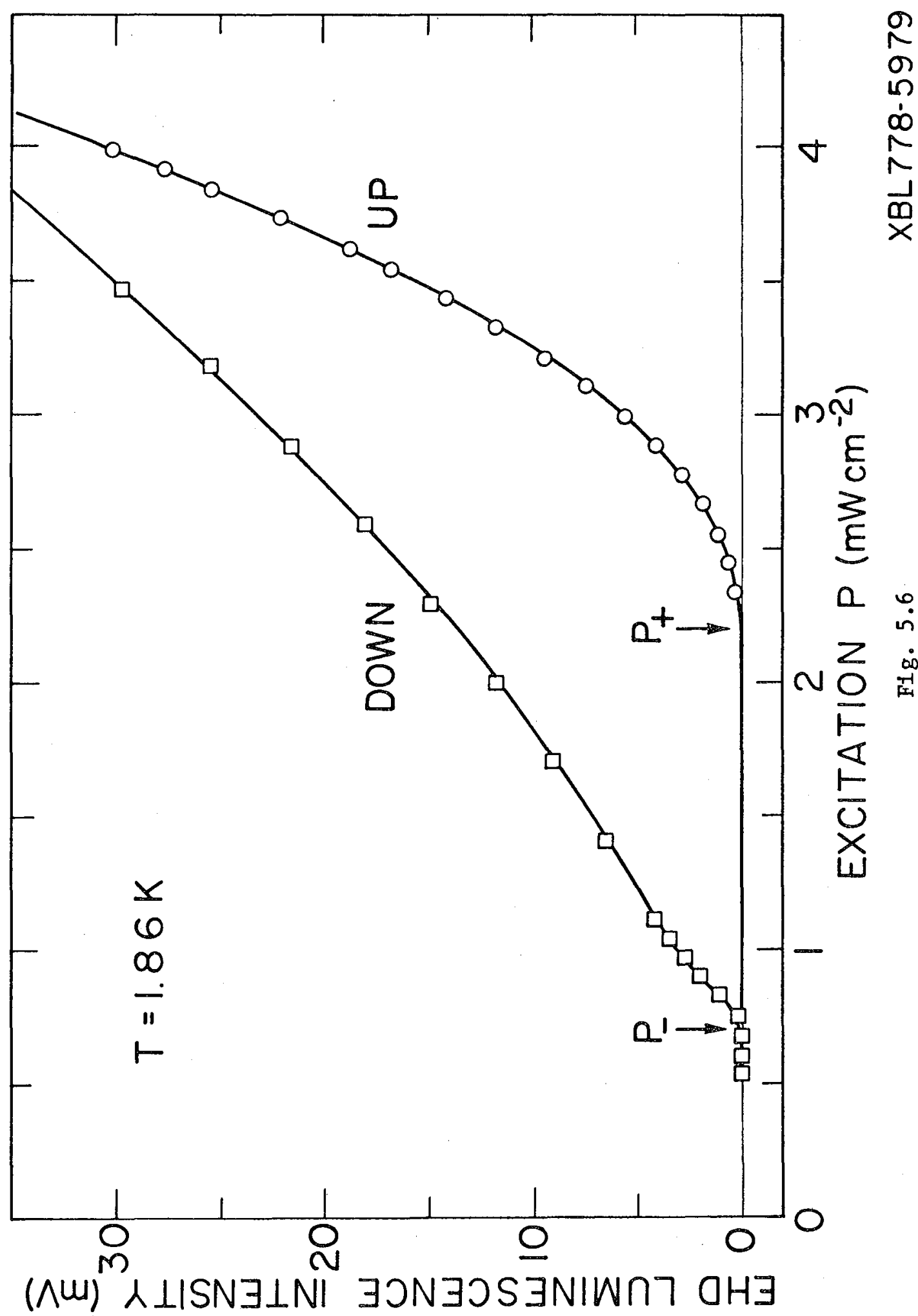


$0000+40461$

$-205-$

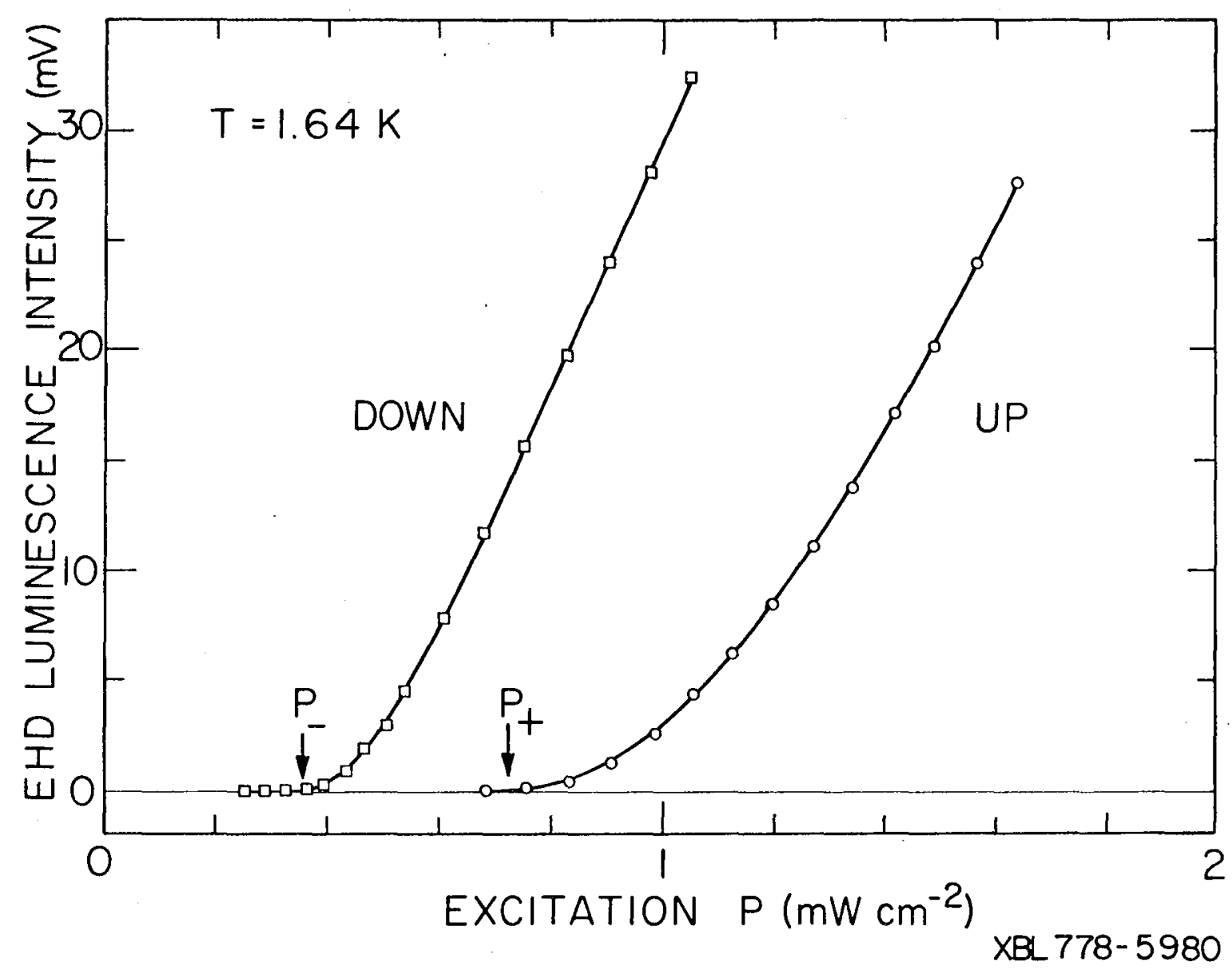

Fig. 5.7 


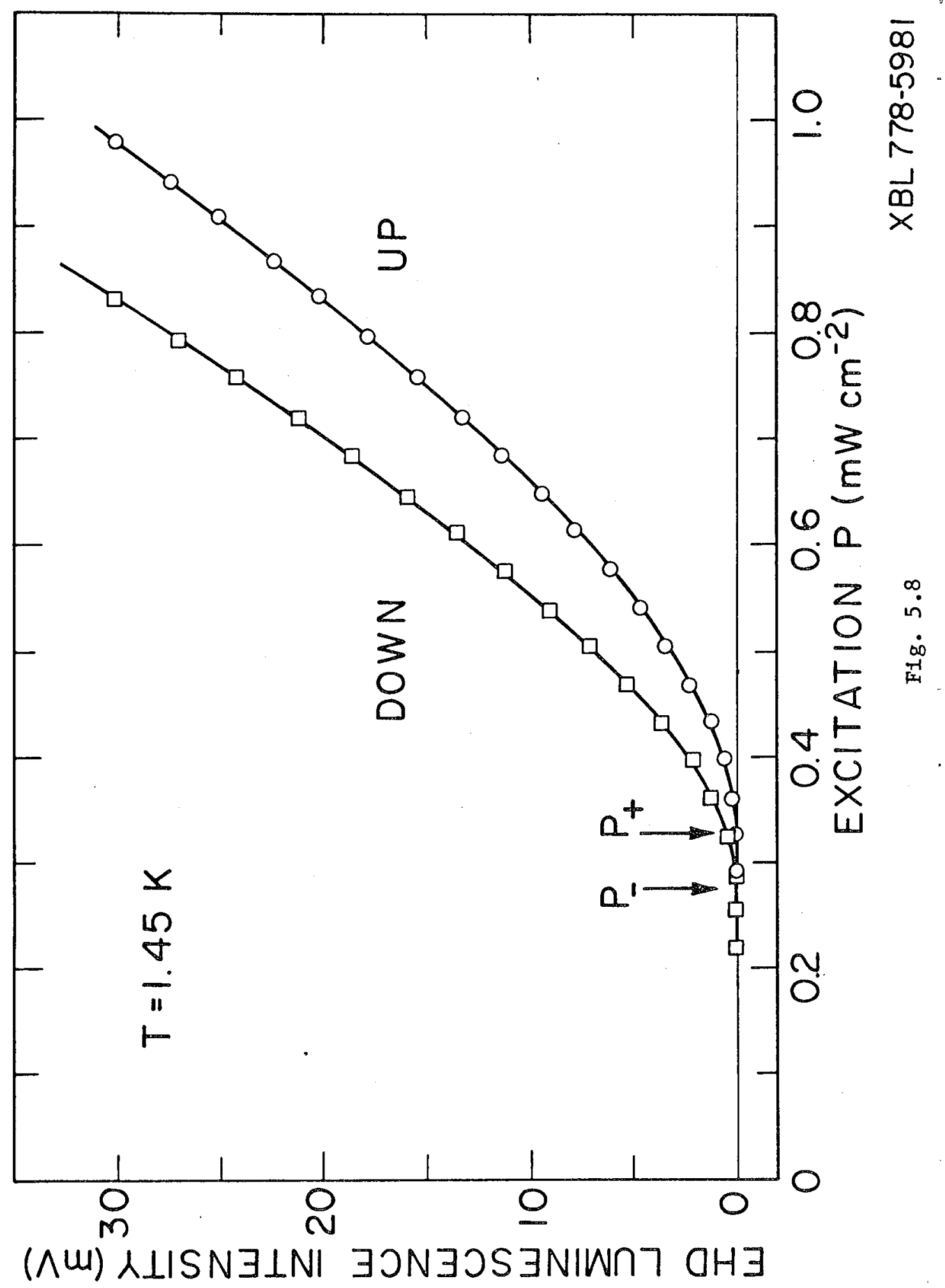




$$
\begin{gathered}
0019+9392 \\
-207-
\end{gathered}
$$

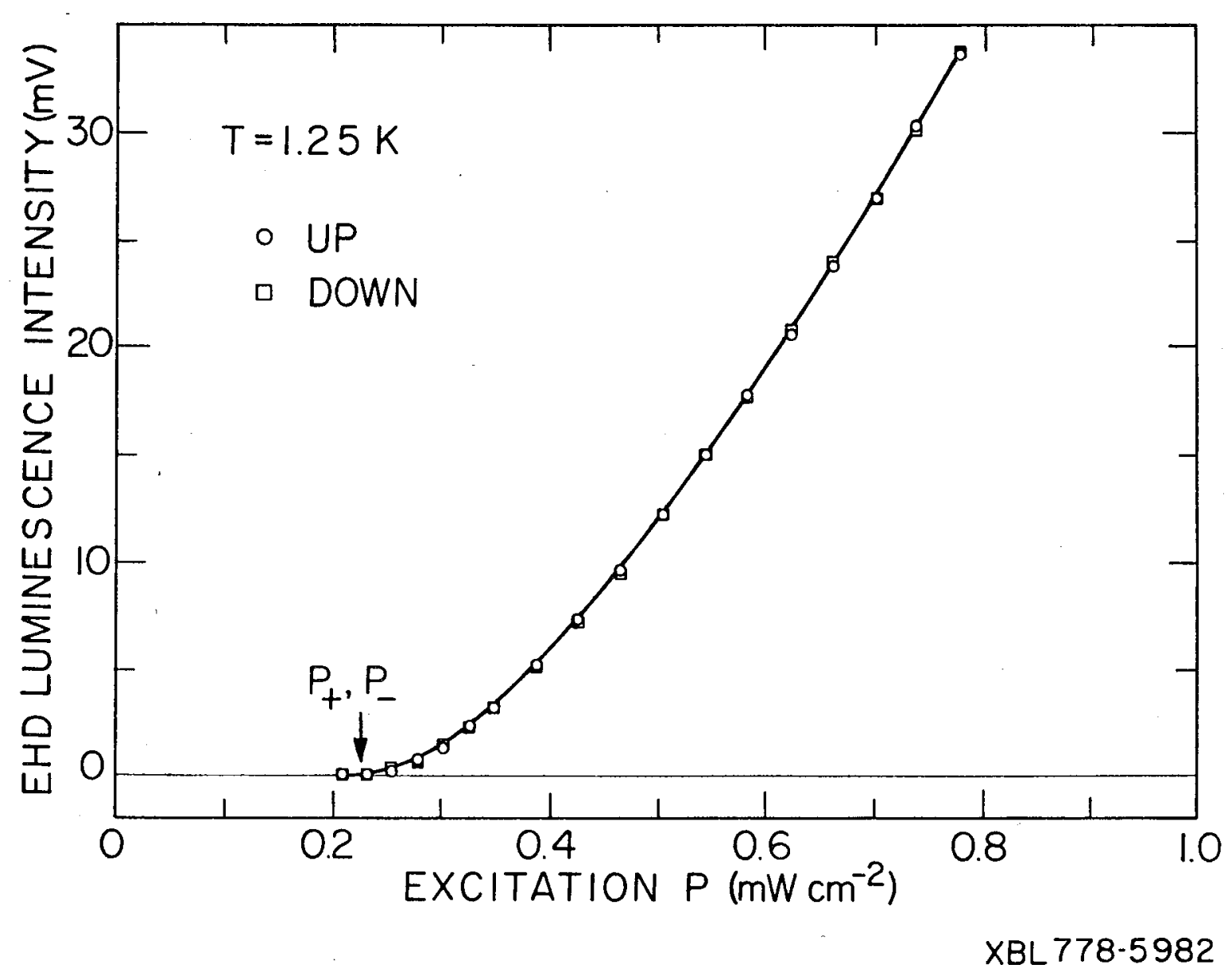

Fig. 5.9 


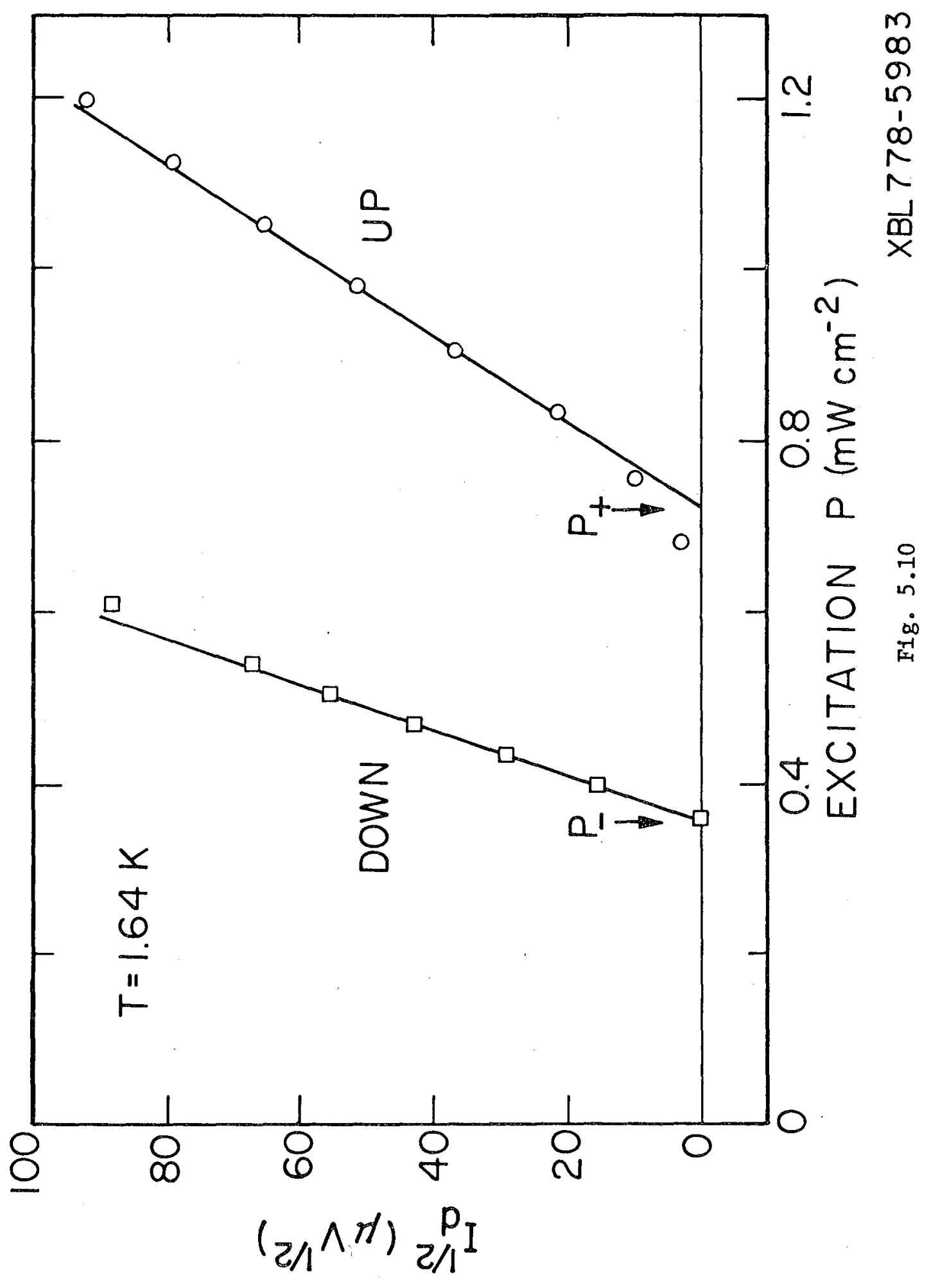


00,090498

$-209-$

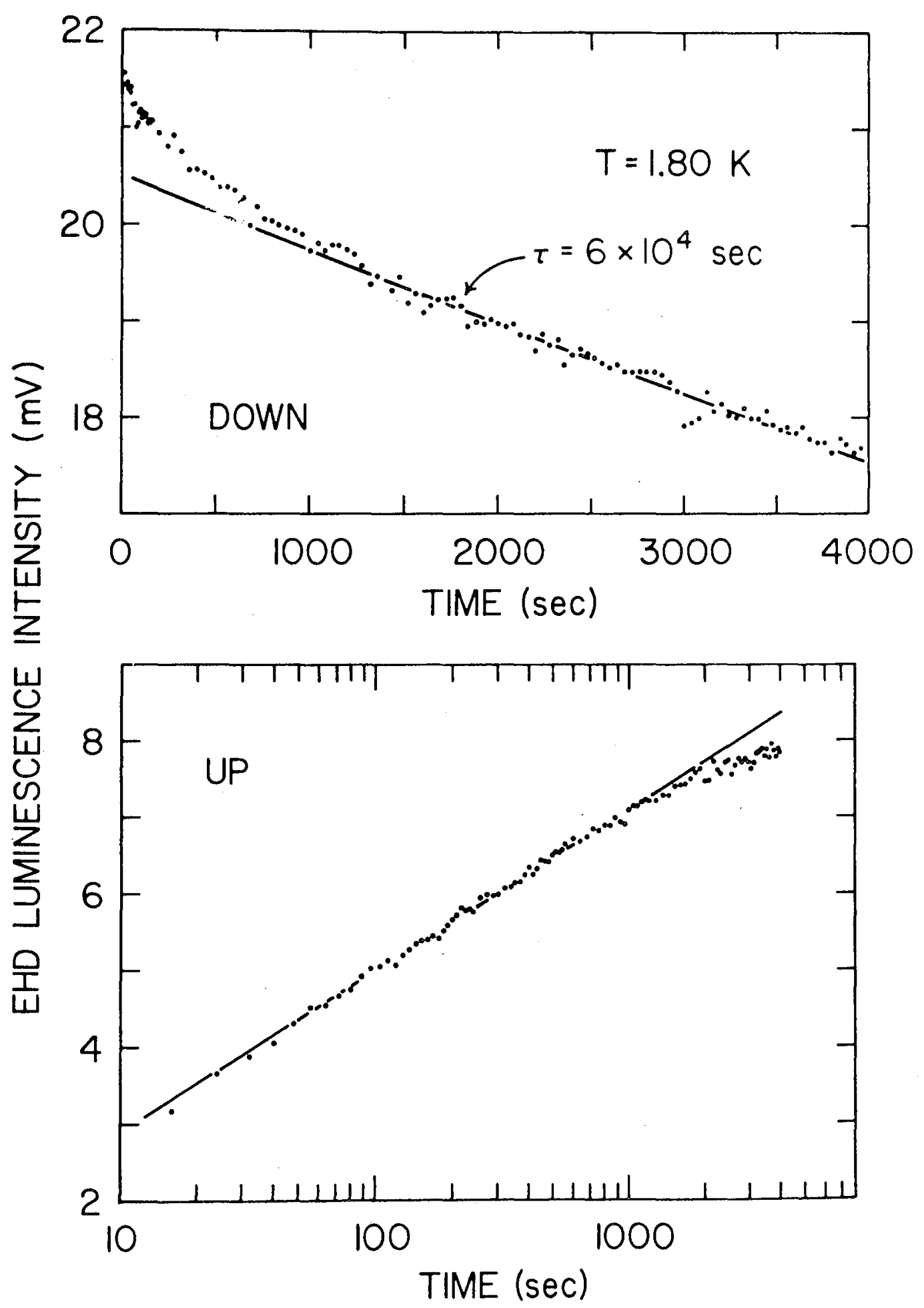

XBL 778-2780

F1g. 5.11 


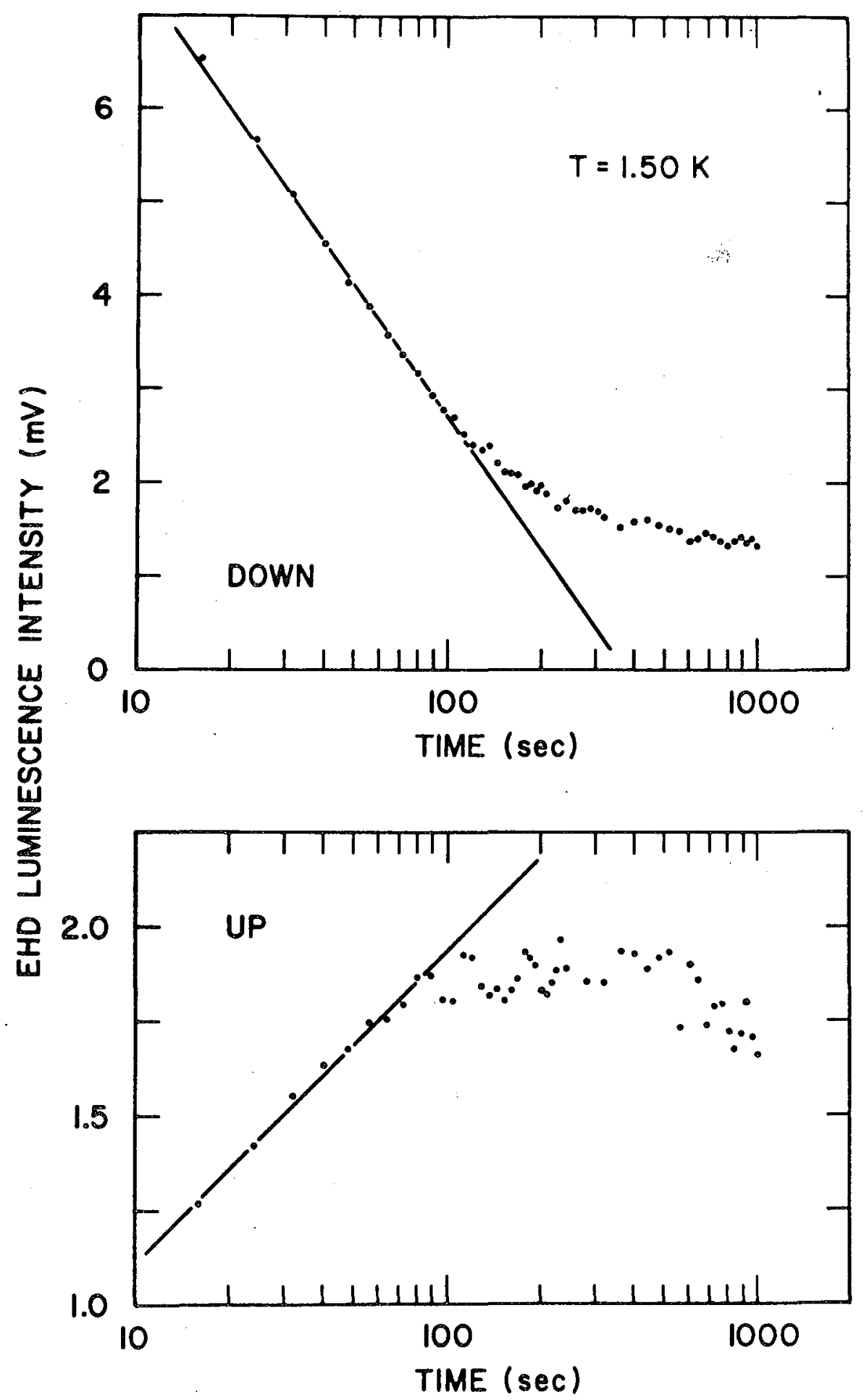

XBL $778-2770$

Fig. 5.12 
0000400404

$-211-$

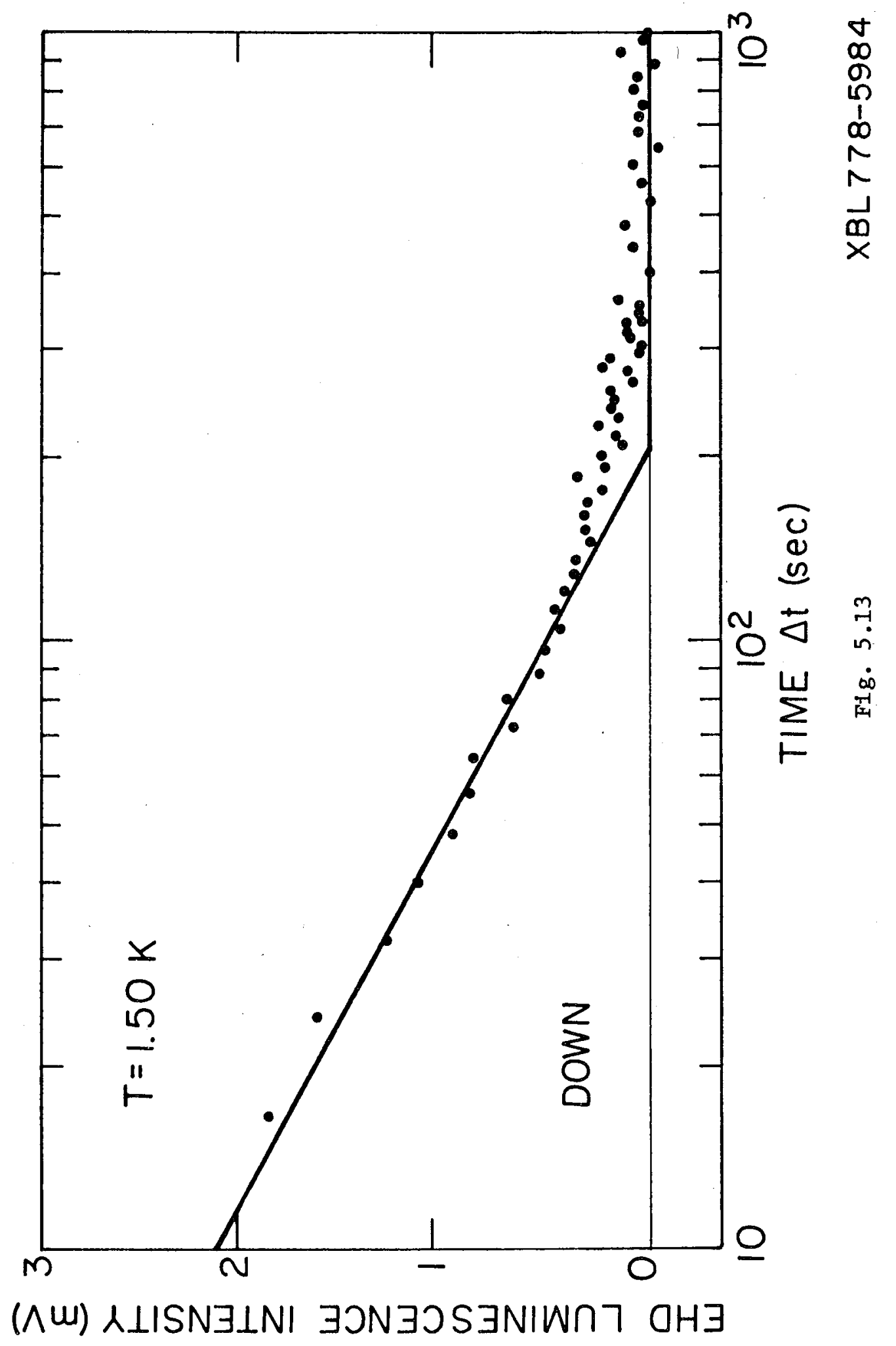


$-212-$
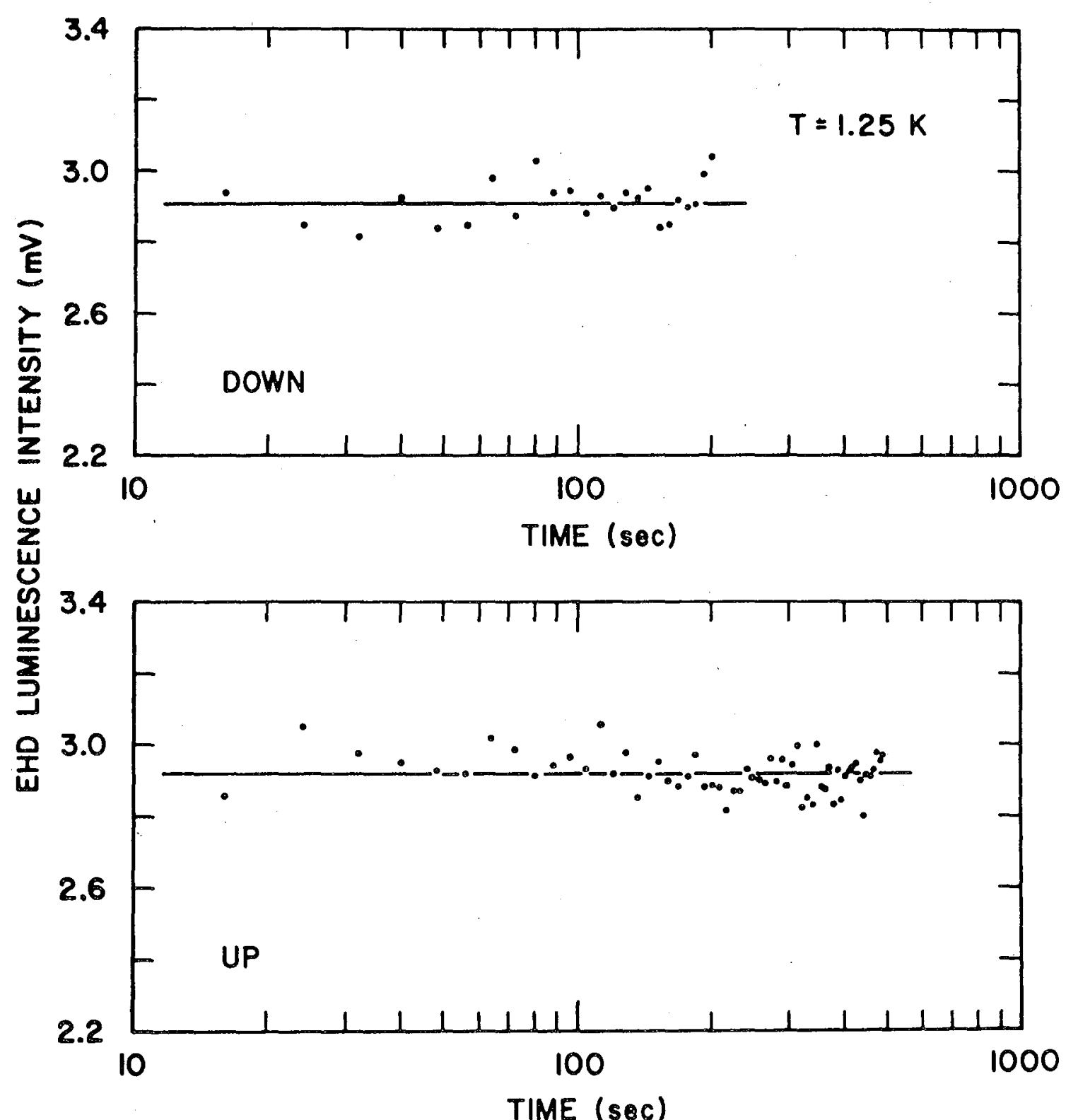

TIME (soc)

XBL 778-2772

FIg. 5.14 


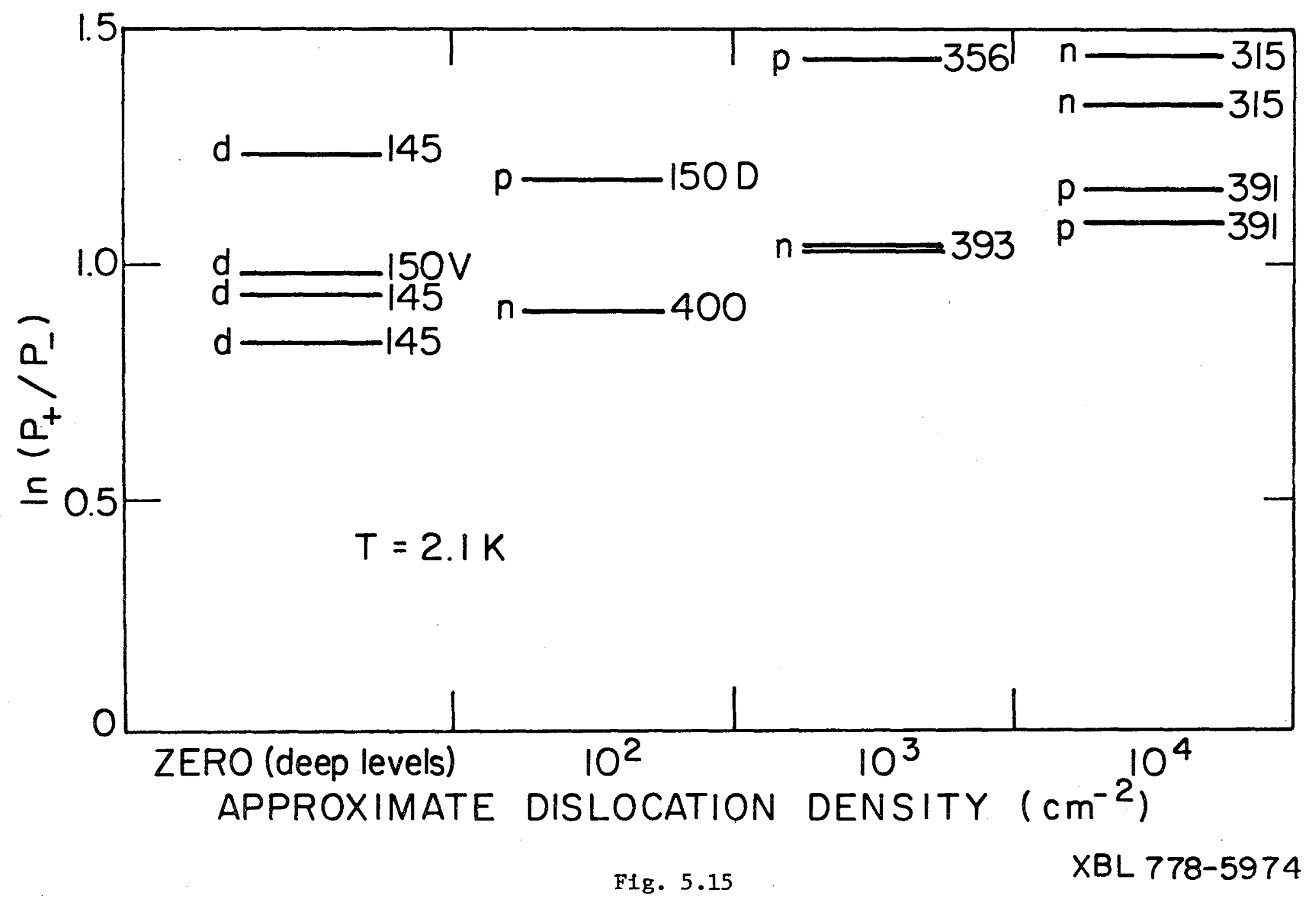




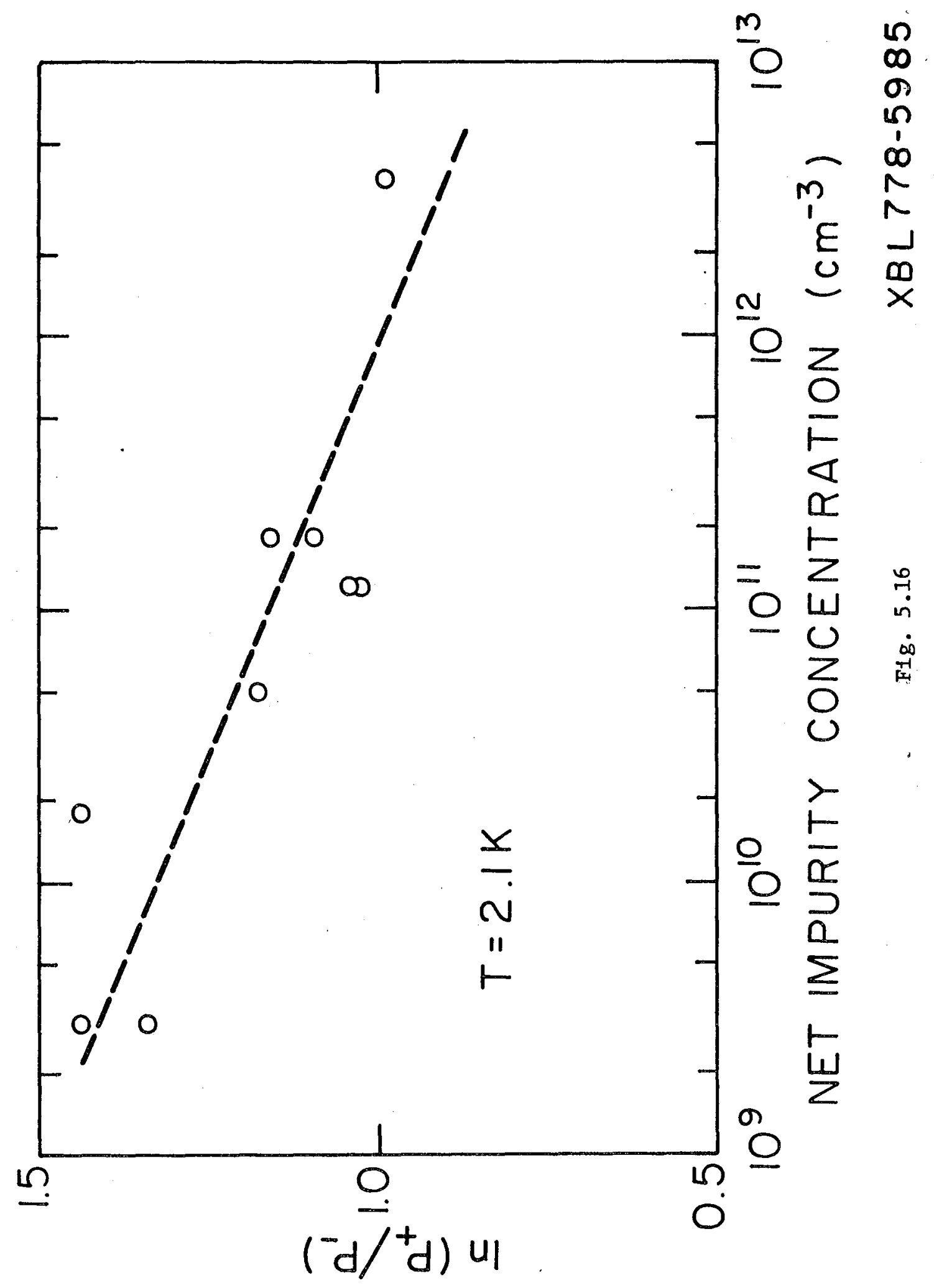



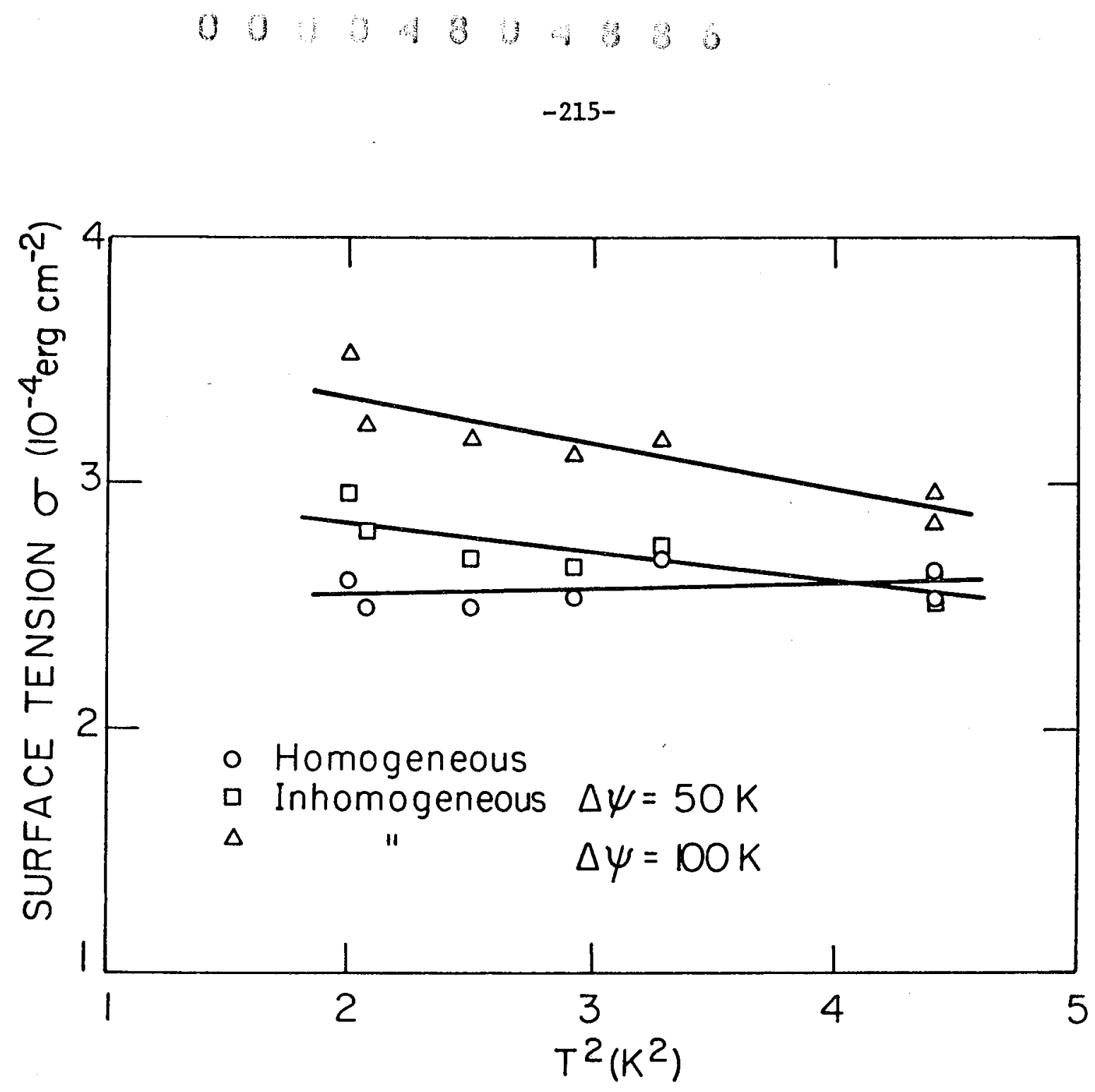

XBL 778-5986

Fig. 5.17 


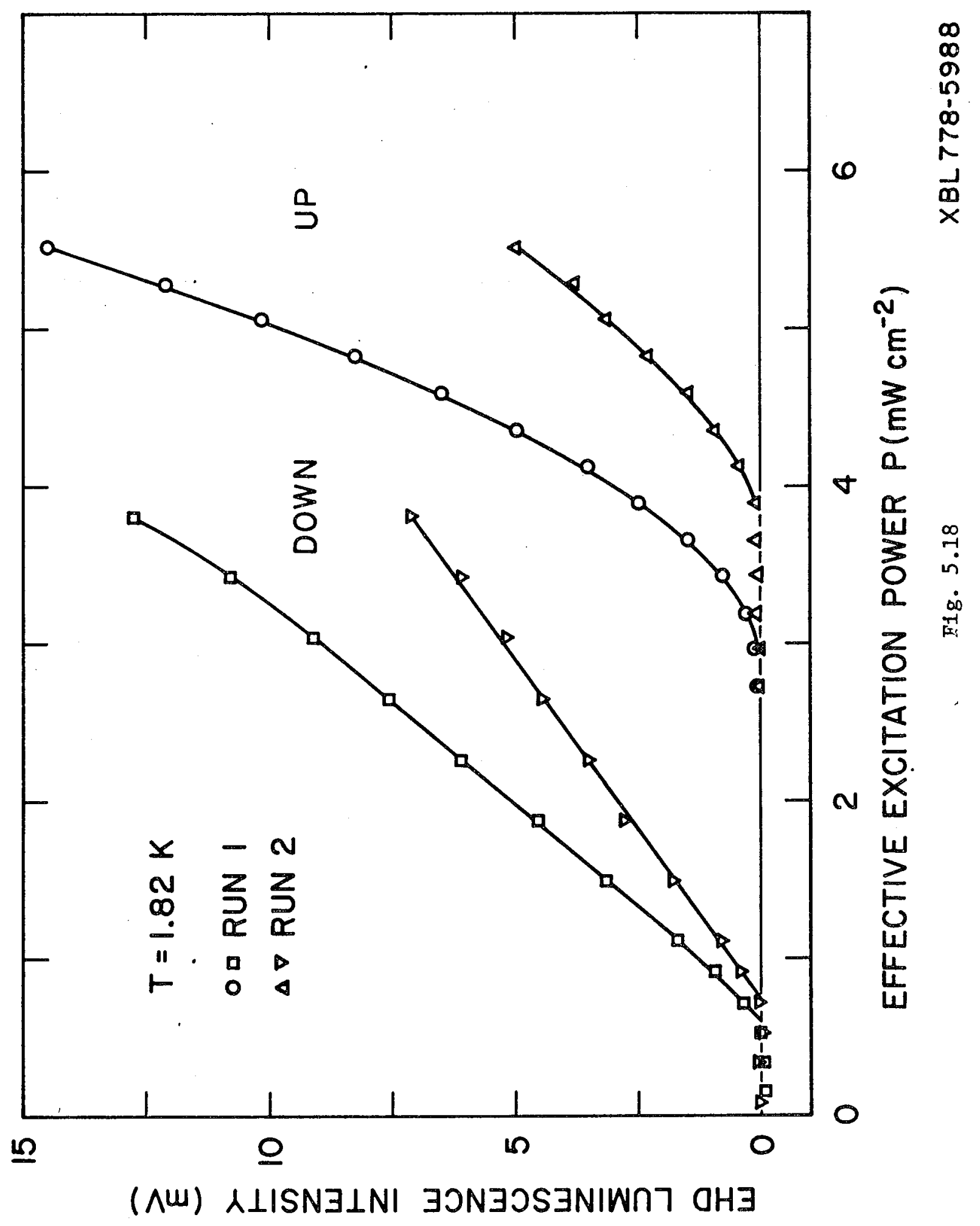


$00+4.40897$

$-217-$

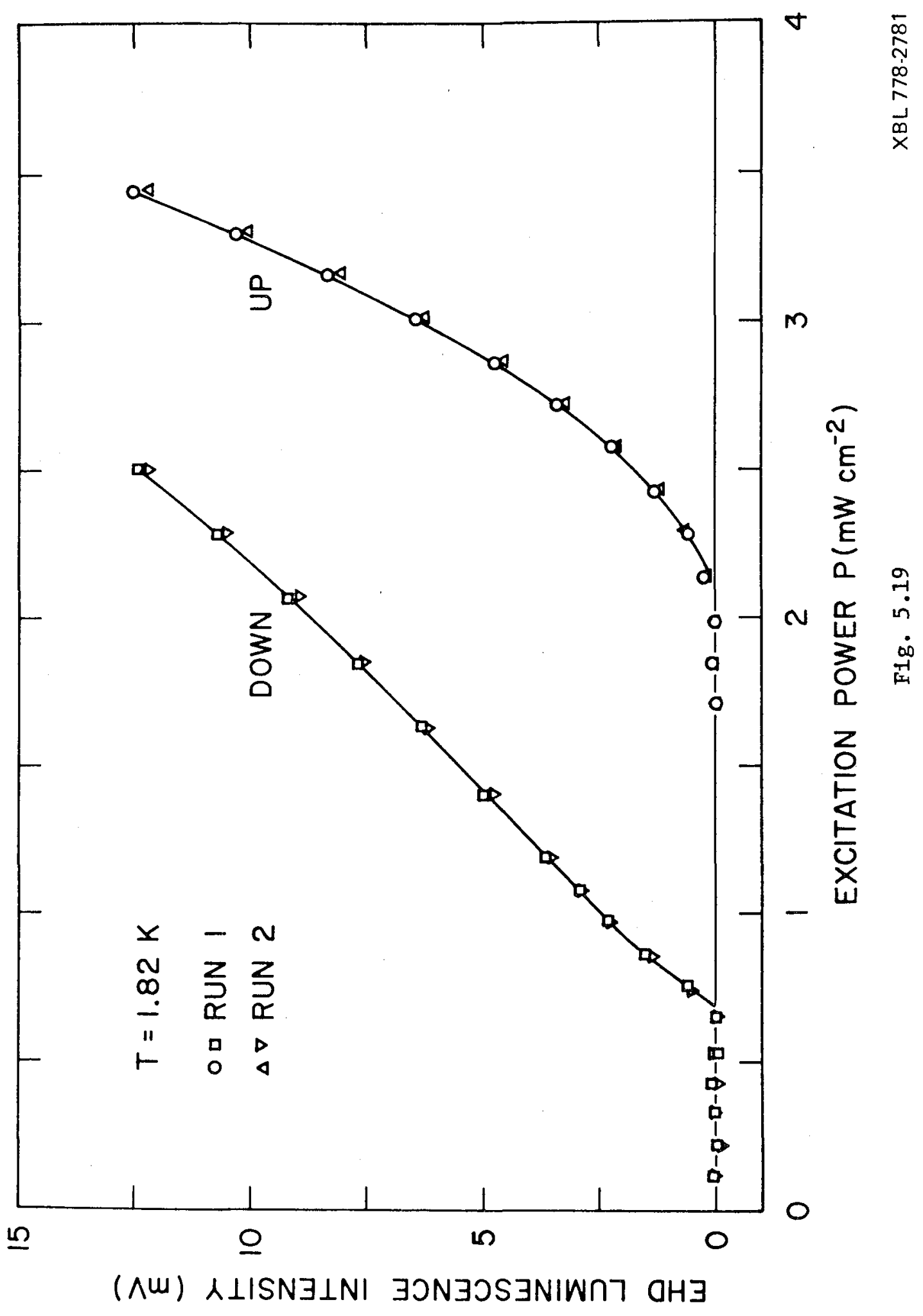




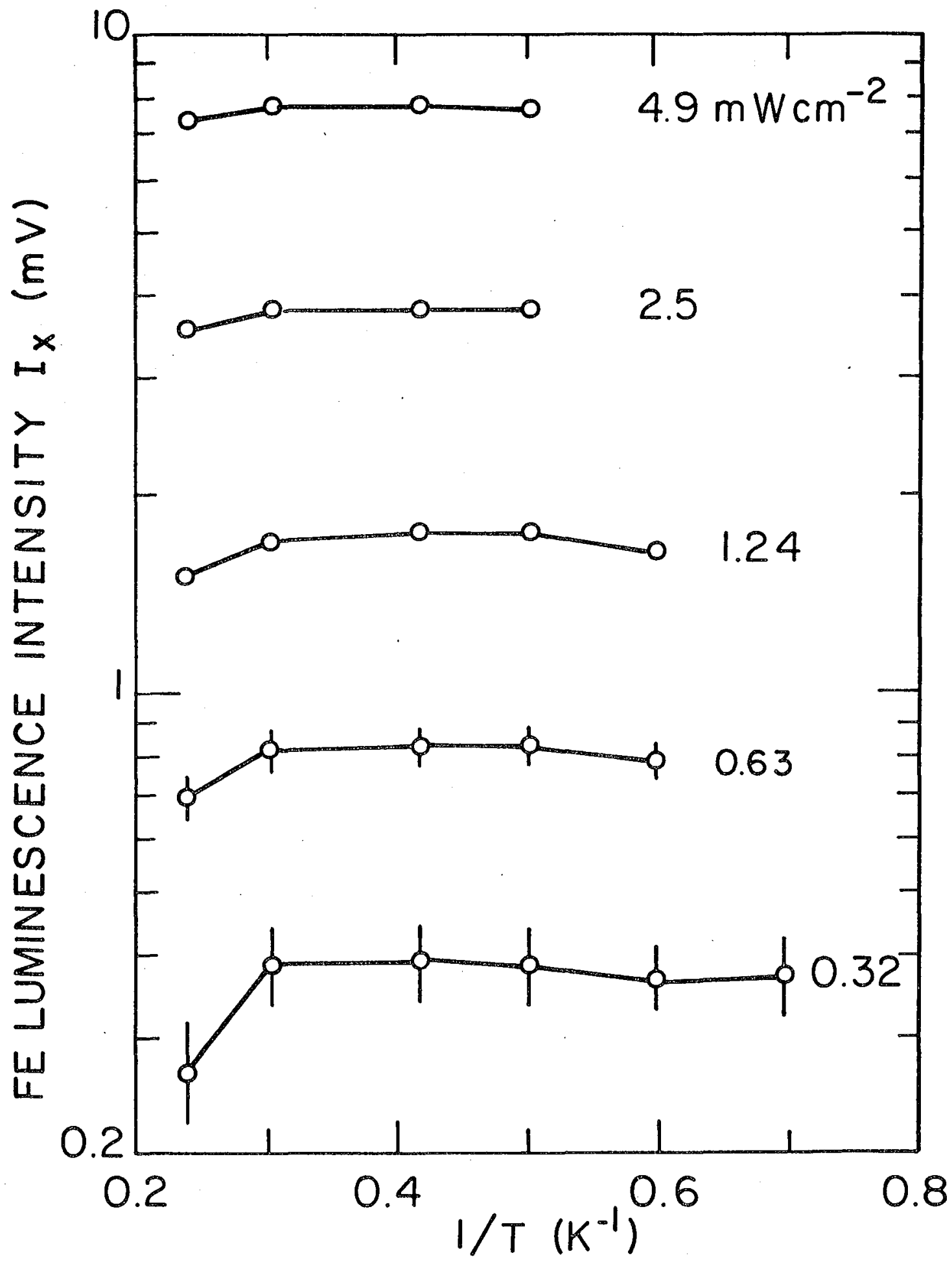

XBL77 8-5989

F1g. 5.20 


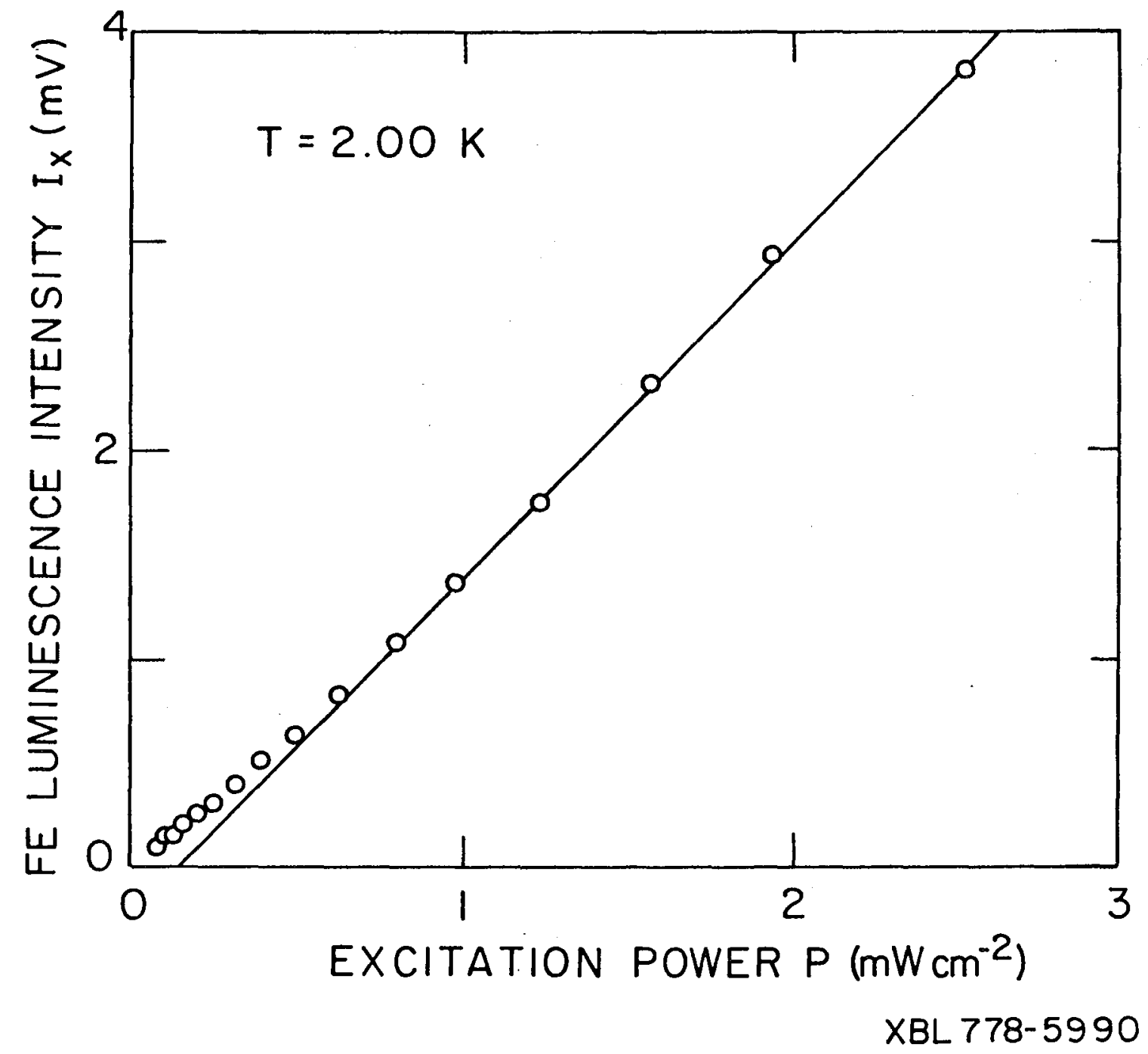

Fig. 5.21 


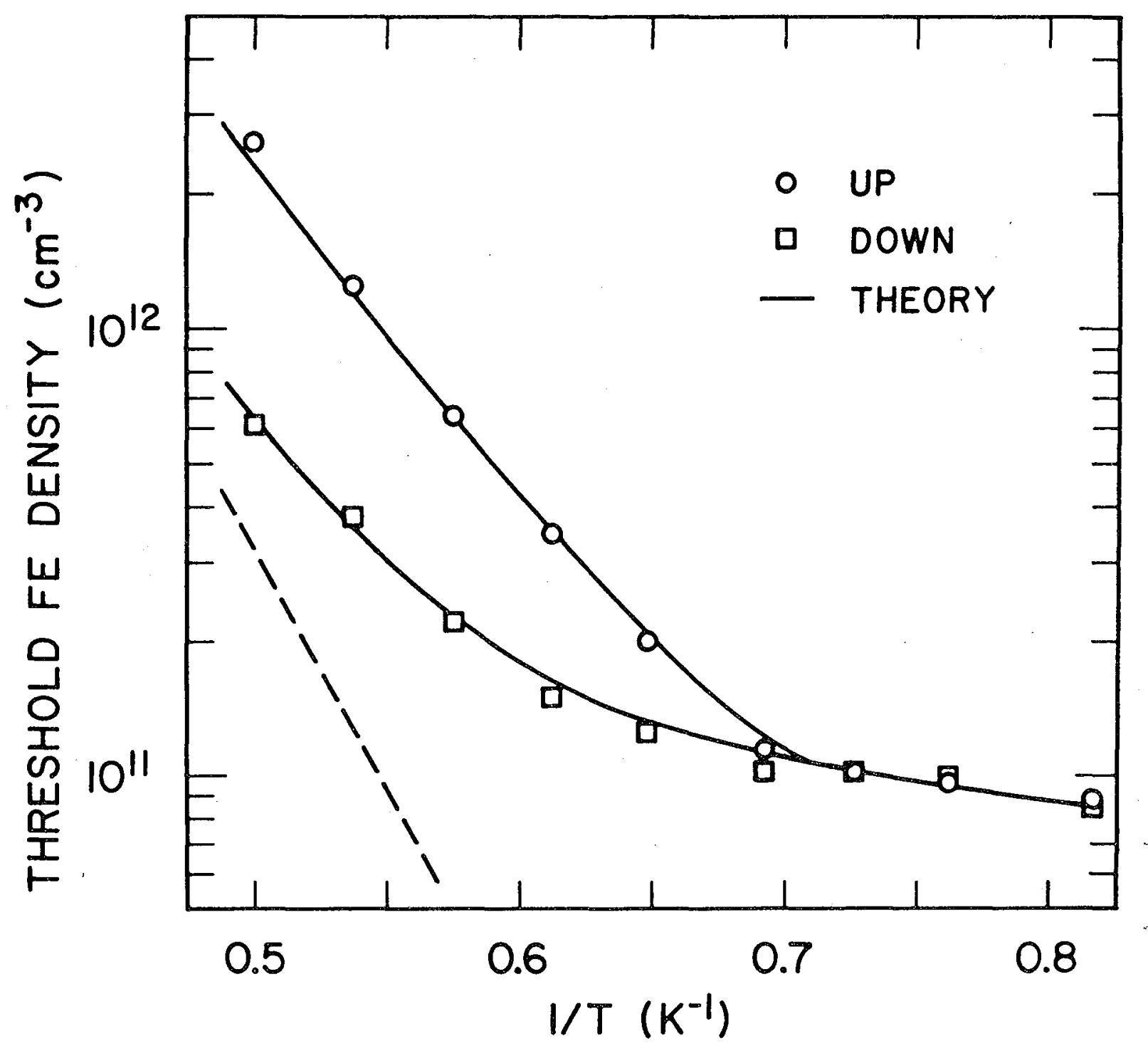

XBL 778.2773

F1g. 5.22 
This report was done with support from the United States Energy Research and Development Administration. Any conclusions or opinions expressed in this report represent solely those of the author(s) and not necessarily those of The Regents of the University of California, the Lawrence Berkeley Laboratory or the United States Energy Research and Development Administration. 